\title{
CHEMICAL GENETIC ANALYSES OF COMPOUNDS DERIVED FROM FEIJOA FRUIT
}

By

Mona Mokhtari

\begin{abstract}
A thesis
submitted to the Victoria University of Wellington

in fulfilment of the requirements for the degree of

Doctor of Philosophy
\end{abstract}

Victoria University of Wellington 



\begin{abstract}
Nature has been a rich source of pharmaceutical compounds, producing $80 \%$ of our currently prescribed drugs. The feijoa plant, Acca sellowiana, is classified in the family Myrtaceae, native to South America, and currently grown worldwide to produce feijoa fruit. Compounds with anticancer, anti-inflammatory, antibacterial and antifungal activities have been isolated from feijoa; however, the diversity of these compounds is not known nor is the mechanism of action of any of these compounds. I hypothesized that identifying compounds in novel feijoa cultivars would improve our understanding of the chemical diversity of antifungal compounds in feijoa and determining the antifungal mechanism of action of feijoa compounds would provide insight into the pharmaceutical potential of these compounds. First, GC-MS analyses were used to obtain an unbiased profile of 151 compounds from 16 cultivars of feijoa, of which six were novel cultivars. Multivariate analysis distinguished 18 compounds that were significantly and positively correlated to antifungal activity based on growth inhibition of Saccharomyces cerevisiae, of which seven had not previously been described from feijoa. Two novel cultivars were identified as the most bioactive cultivars, and the compound 4-cyclopentene-1,3-dione found in a couple of cultivars was potently antifungal against human pathogenic isolates of four Candida species. Second, chemical genetic analyses were used to investigate the mechanism of action of estragole, an antifungal compound previously isolated from feijoa. The chemical genetic profile of estragole was distinct from that of other known antifungal compounds, suggesting the mechanism of action of estragole has a novel antifungal mechanism. Third, chemical genetic analyses were used to investigate the mechanism of action of an ethanol adduct of vescalagin (EtOH-vescalagin) isolated from feijoa. We showed EtOH-vescalagin is antifungal against human pathogenic strains. Genome-wide chemical genetic analyses of EtOH-vescalagin indicated antifungal activity is mediated by disruptions of iron homeostasis, zinc homeostasis and retromer
\end{abstract}


recycling through iron chelation. Overall, these results indicate the chemical and biological value of feijoa as a source of antifungal drugs. 
Dedicated to:

My beloved husband and best friend "Ali "

for his endless support. 



\section{Acknowledgements}

I wish to acknowledge a number of people who have given invaluable support during this study:

I am indebted to my supervisor, Dr Andrew Munkacsi for his support, expertise, systematic supervision as well as his invaluable advice and critical evaluations provided. I could not get it done without your support Andrew. I would also like to faithfully acknowledge my secondary supervisor, Dr. Robert Keyzers who kindly supported me through this project with his valuable chemistry perspectives, which contributed greatly to my thesis. I thank you both for your unique and tremendous assistants.

I would also like to acknowledge the assistance and support rendered by Prof Paul Atkinson for his huge support and consideration in my difficult times. Special thanks to Prof John Miller and Ben Jones for kindly helping me in anti-cancer assays, which also allowed me to learn a lot about tissue culturing. I would also like to thank Dr David Ackerley and Alistair Brown for their assistance in several antibacterial assays and also manuscript preparation.

Sincere thanks to my dear friends Mohammad Nekooie for all of his generosity, assistance and kindness at all times including the last stressful days before my submission and also Dinindu Senanayake for all of his scientific and personal support whenever I needed it.

My heartfelt appreciation also goes to Callaghan Innovation, especially Dr. Stephen Bloor for providing some of the feijoa compounds used in this thesis and even more support in future collaboration. I would like to especially thank Nigel Ritson and Foretaste Feijoa Fruit company for their great cooperation---it was my pleasure to work on your feijoa cultivars Nigel. I would also like to thank Dr David Gresham from New York University who generously collaborated with us on the barcode sequencing and data analysis, as well as Prof David Eide from the University of Wisconsin for providing yeast strains. To Michael Jackson, thank you so much 
for helping me learn and conduct multivariate statistical analyses. I would like to thank Mathew Cairns for his patience and help running the atomic absorption spectrometer.

I would also like to acknowledge and thank Victoria University of Wellington, notably the International Students Support Office, the Faculty of Science especially Patricia Stein, and the Scholarship Office for providing the scholarship and support during my study. I would not have attained my goals without their support and generosity. I also acknowledge all Chemical Genetics Laboratory members who helped me during my project including Dr. James Mathew and Jeffrey Sheridan for his useful comments on my thesis and his time.

Last but not least, I am thankful to my dear husband, Ali, for his continued support, patience and understanding during the course of my study. Ali, without your endless support and push, this work could not have been completed. I also thank my dear parents as they always encouraged and supported me in all aspects, which made me indebted to you for always. I also thank my three sisters and their cute kids for all of their emotional support and kindness.

I dedicate this thesis to my dear parents who have been inspirational in encouraging me to look to greater heights in search of knowledge and excellence. My warm apology to those whom I have omitted to mention; thank you very much.

\section{YOURS FAITHFULLY}

Mona Mokhtari 
ABSTRACT

Chapter 1 Literature review ..................................................................................................................... 1

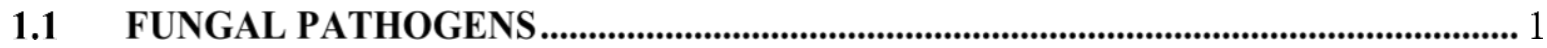

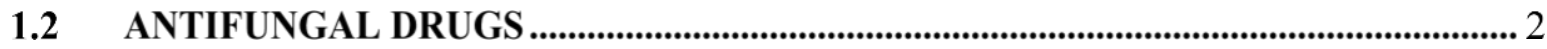

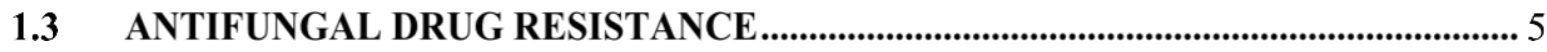

1.4 NATURAL PRODUCTS AND DRUG DISCOVERY ...................................................... 6

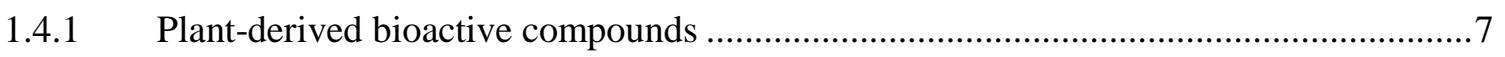

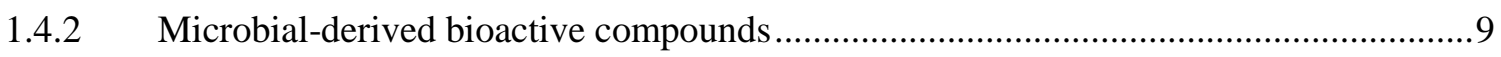

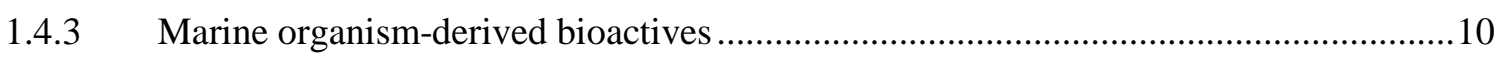

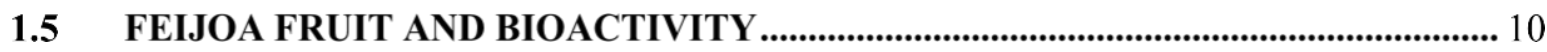

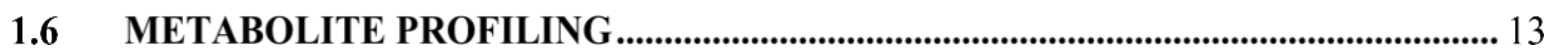

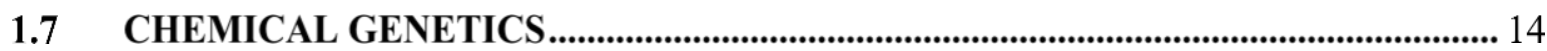

1.7.1 Yeast as a model organism for bioactivity and chemical genetics ................................14

1.7.2 Non-essential gene deletion libraries ......................................................................... 16

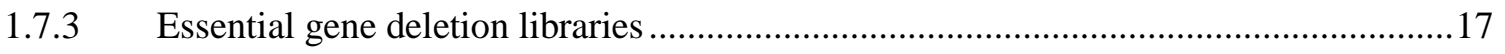

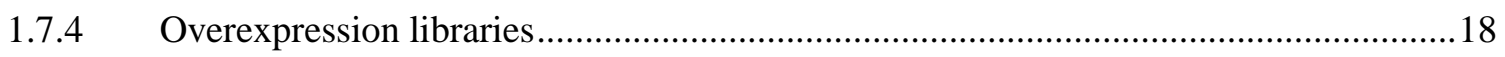

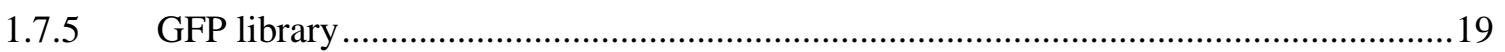

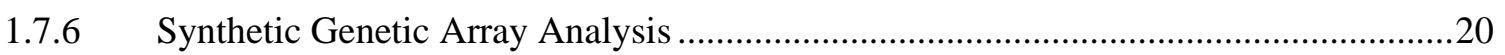

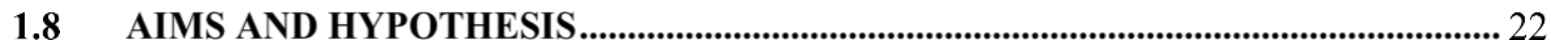

Chapter 2 Bioactivity-Guided Metabolite Profiling of Feijoa Cultivars.............................223

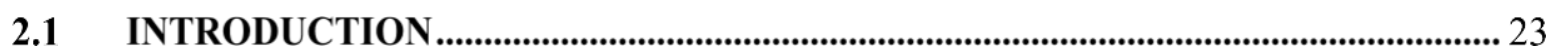

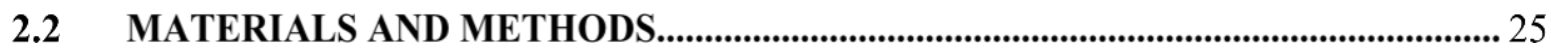

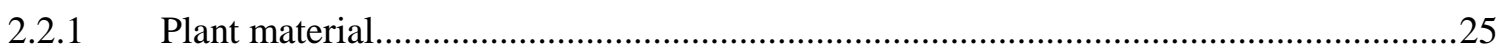

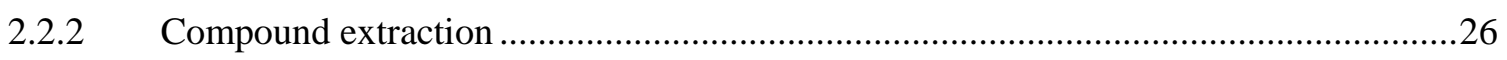

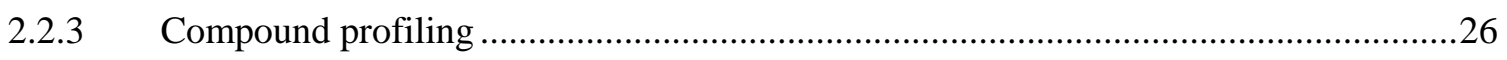

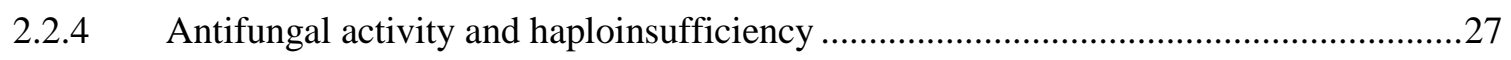

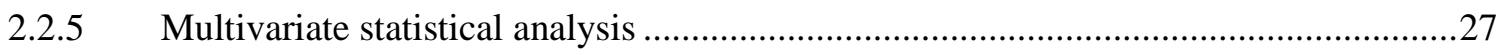

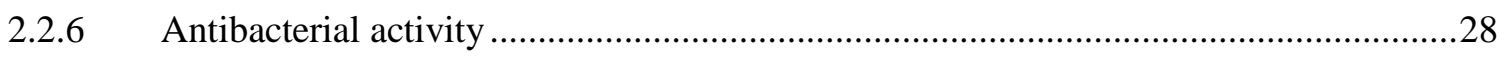

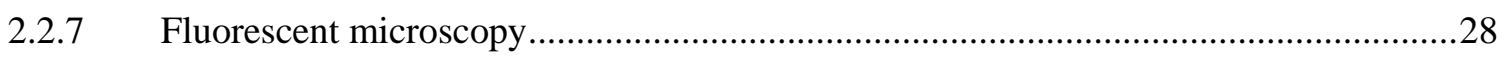

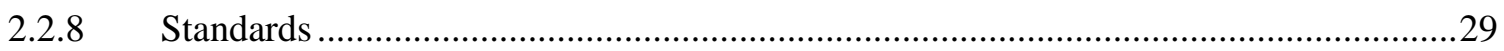

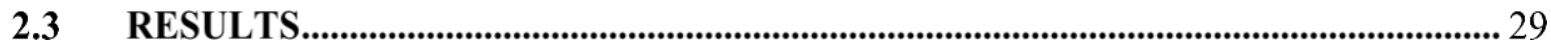




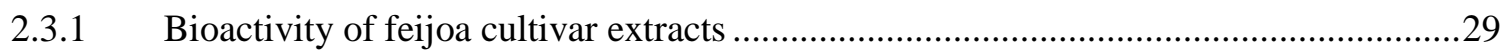

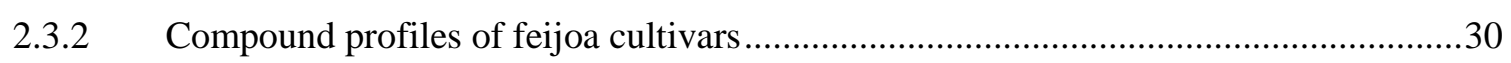

2.3.3 Multivariate analysis of cultivars, bioactivity, and compounds ......................................

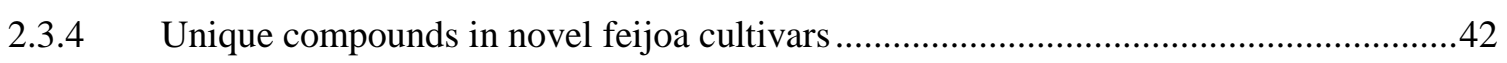

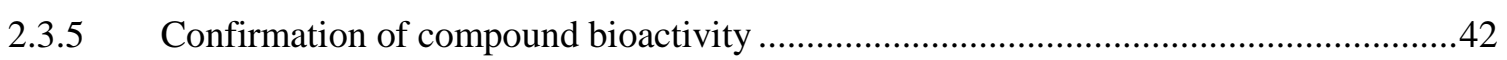

2.3.6 Antifungal activity of feijoa cultivars and compounds against human pathogens ........43

2.3.7 Antifungal mechanism of action of 4-cyclopentene-1-3-dione.....................................45

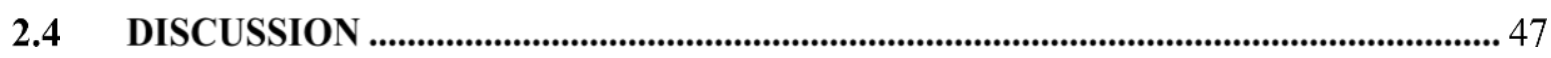

Chapter 3 Elucidating the antifungal mechanism of action of EtOH-vescalagin..............53

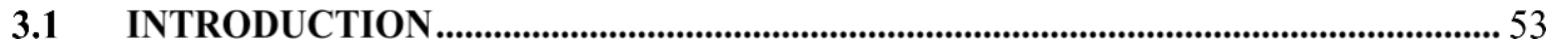

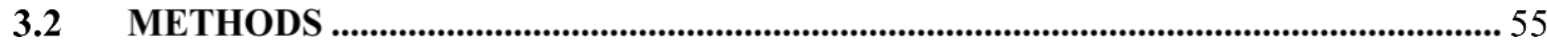

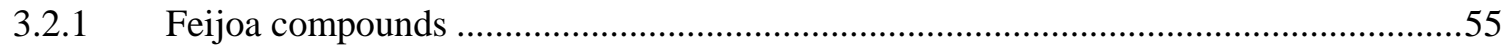

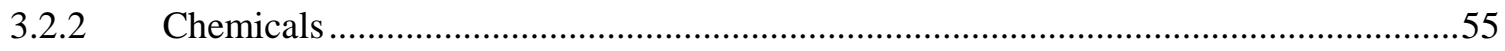

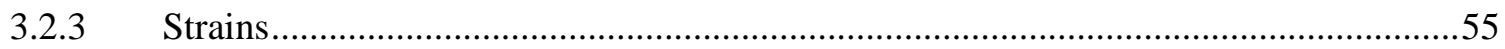

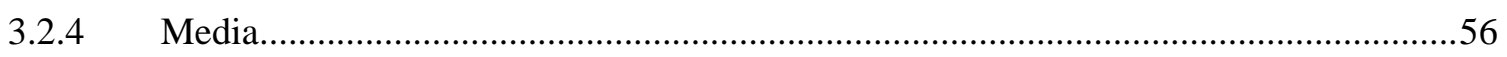

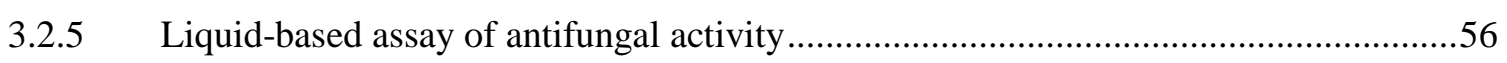

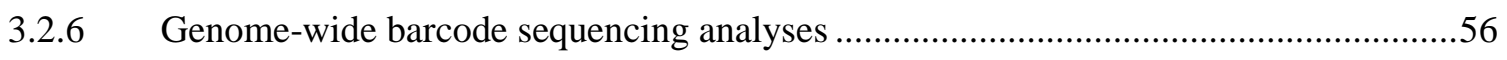

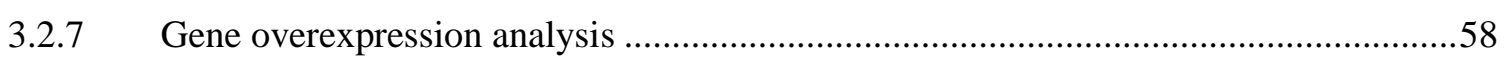

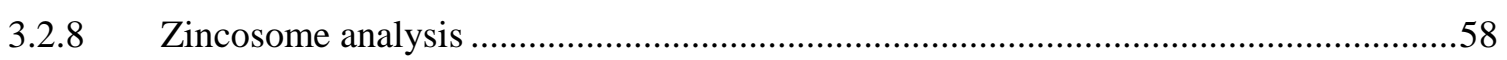

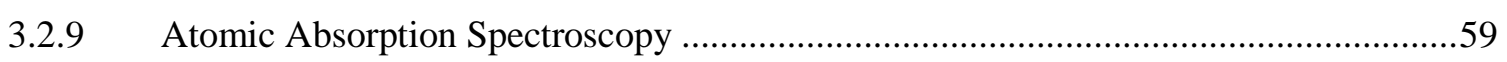

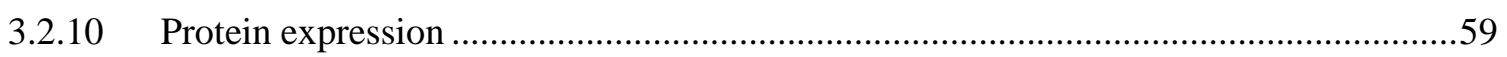

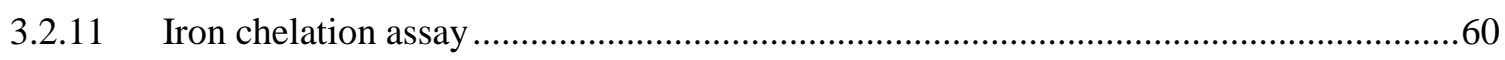

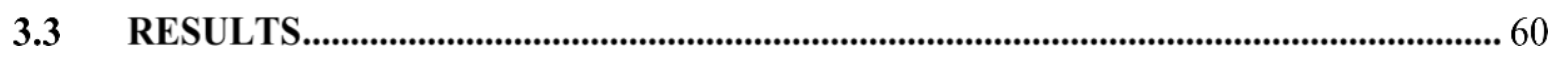

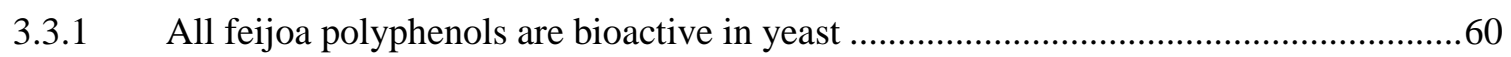

3.3.2 EtOH-vescalagin is antifungal against pathogenic Candida strains..............................61

3.3.3 HIP-HOP analysis to the mode of action of EtOH-vescalagin.....................................62

3.3.4 HOP analysis identifies genes buffering the mechanism of EtOH-vescalagin .............63

3.3.5 HIP analysis of Et-OH-vescalagin identifies sensitive HET strains.............................68

3.3.6 Gene Ontology analyses distinguish metal homeostasis and the retromer complex .....71

3.3.7 Iron, zinc and copper supplementation impacts EtOH-vescalagin bioactivity ...............72

3.3.8 Zinc and iron mediate retromer-specific sensitivity to EtOH-vescalagin .......................77

3.3.9 Vacuolar storage of zinc buffers EtOH-vescalagin toxicityn ......................................79

3.3.10 EtOH-vescalagin treatment phenocopies zinc supplementation and is not a zinc chelator 81

3.3.11 EtOH-vescalagin increases intracellular zincosomes .82 
3.3.12 EtOH-vescalagin increases intracellular zinc levels and decreases intracellular iron levels 84

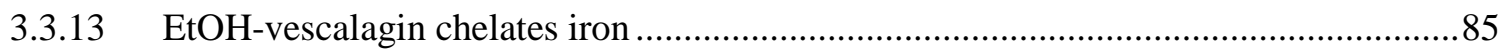

3.3.14 EtOH-vescalagin increases expression of iron transporters ......................................... 87

3.3.15 EtOH-vescalagin increases expression of vacuolar zinc transporter and decreases

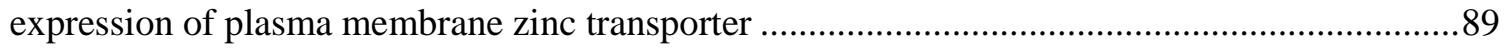

3.3.16 EtOH-vescalagin disrupts retromer function .................................................................

3.3.17 Deletion-based GFP analyses strengthen the iron-zinc-retromer mechanisms of EtOHvescalagin.....

3.3.18 Overexpression analyses strengthen the iron-zinc-retromer mechanisms of EtOHvescalagin......

3.3.19 EtOH-vescalagin disrupts mitochondrial function and respiration .............................107

Chapter 4 Elucidating the Antifungal Mechanism of Action of Estragole ...................... 117

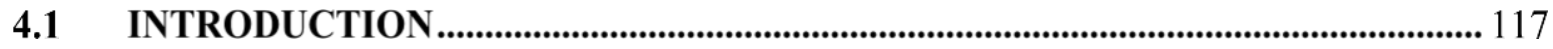

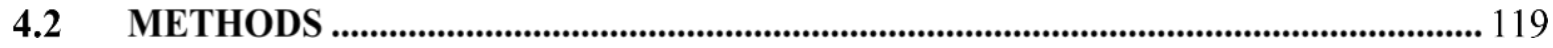

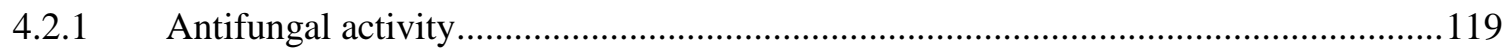

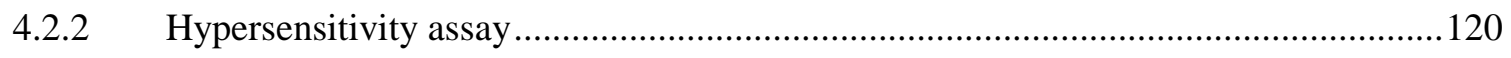

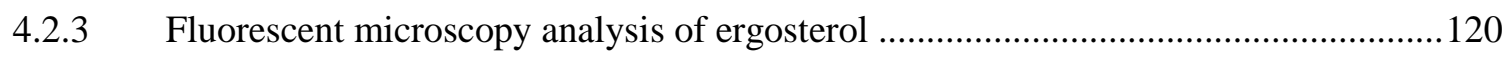

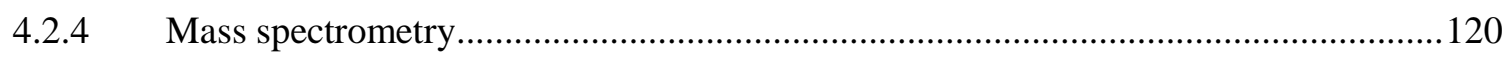

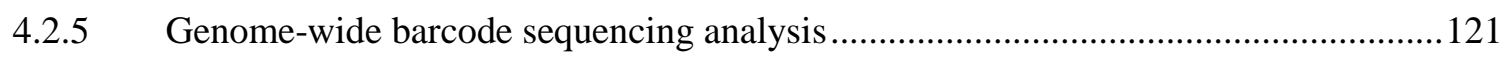

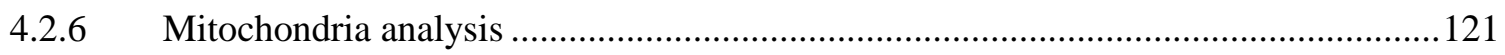

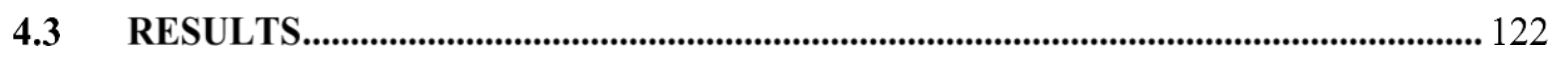

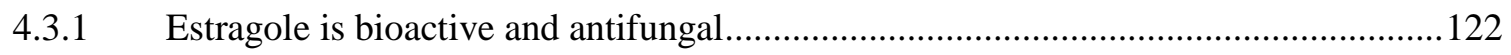

4.3.2 The ketoconazole drug target ERG11 is not the target of estragole .............................123

4.3.3 Ergosterol biosynthetic genes are not the target of estragole .......................................125

4.3.4 Ergosterol homeostasis is not the target of estragole ................................................126

4.3.5 Estragole does not target ergosterol in the plasma membrane ...................................127

4.3.6 The echinocandin target FKS1 is not the target of estragole......................................128

4.3.7 The LCB1 target of myriocin is not the target of estragole........................................129

4.3.8 The CHS1 target of polyoxin is not the target of estragole ........................................130

4.3.9 Estragole mechanism is different from Flucytosine .................................................131

4.3.10 Synergy of estragole and established antifungal drugs .............................................132

4.3.11 HIP-HOP analysis to determine the mechanism of action of estragole .......................135

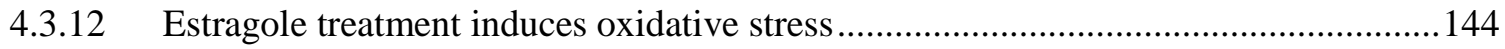

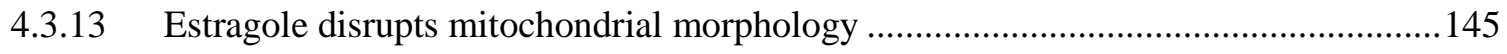

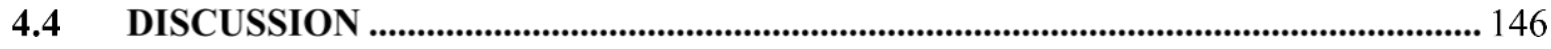


Chapter 5 Implications and future directions........................................................................... 151

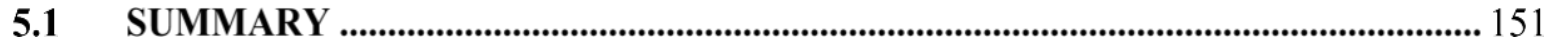

5.2 IMPLICATIONS ON HUMAN DISEASES AND THERAPEUTICS.......................... 153

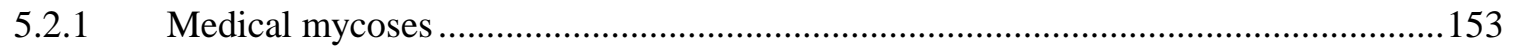

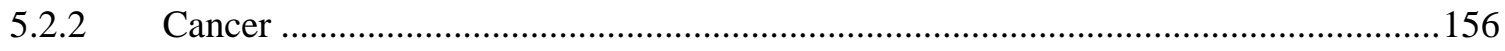

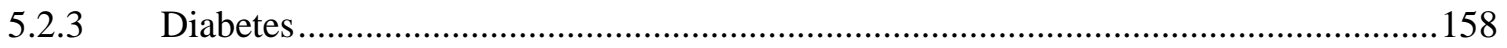

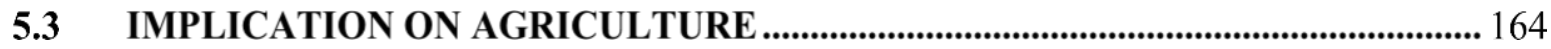

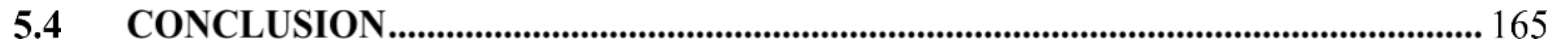

References............................................................................................................................................................ 167

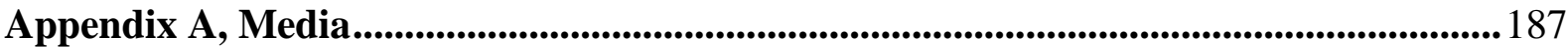




\section{List of figures}

Figure 1-1. Anti-cancer drugs approved between 1940 and 2014 ..................................................... 7

Figure 1-2. Feijoa fruit (Acca sellowiana). .......................................................................................... 11

Figure 1-3. Distribution of studies investigating four types of bioactivity in feijoa. ...................... 13

Figure 1-4. An overview of a chemical genetic interaction................................................................ 16

Figure 2-1. Representative images of feijoa including feijoa tree ......................................................... 24

Figure 2-2. Bioactivity of methanolic extracts $(0.01 \% \mathrm{v} / \mathrm{v})$ of 16 feijoa cultivars based on growth

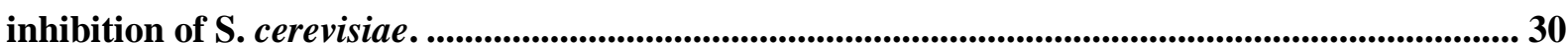

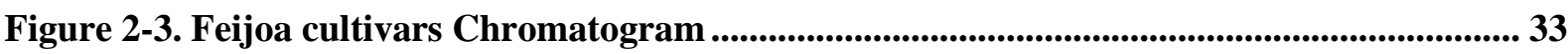

Figure 2-4. Partial least squares regression of (A) feijoa cultivar relative to bioactivity and (B) feijoa compound relative to bioactivity. 40

Figure 2-5. 4-cyclopentene-1,3-dione inhibits chitin synthesis via CHS1 and CHS2..................... 46

Figure 3-1. Structures of vescalagin and EtOH-vescalagin................................................................. 54

Figure 3-2. Polyphenols from feijoa are growth inhibitors in WT yeast at $1 \mu \mathrm{M}$.......................... 61

Figure 3-3. Barcode-sequencing platform.................................................................................................63

Figure 3-4. Fitness plot of HOM library in presence of EtOH-vescalagin..................................... 64

Figure 3-5. Validation of sensitive homozygous deletion strains in presence of EtOH-vescalagin.. 65

Figure 3-6. The $z r t 1 \Delta$ deletion strain grew better in presence of EtOH-vescalagin. ....................... 65

Figure 3-7. Fitness plot of HET library in presence of EtOH-vescalagin. ..................................... 68

Figure 3-8. Validation of 16 sensitive heterozygous deletion strains in presence of EtOH-

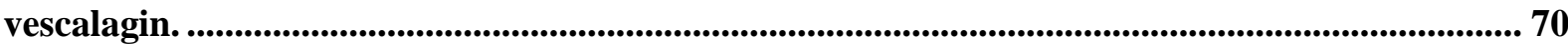

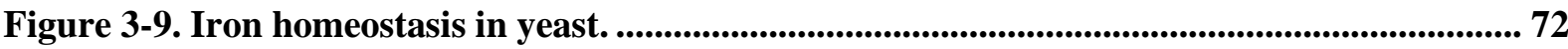

Figure 3-10. Iron supplementation in the presence of EtOH-vescalagin. ......................................... 73

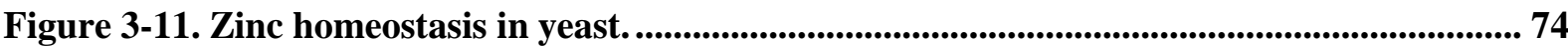

Figure 3-12. Zinc supplementation in the presence of EtOH-vescalagin. ....................................... 75

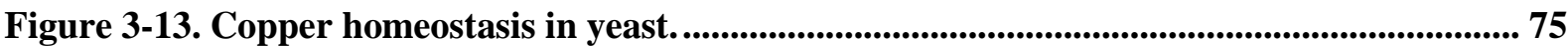

Figure 3-14. Copper supplementation in presence of EtOH-vescalagin. ...................................... 76

Figure 3-15. Retromer deletion strains respond to iron............................................................... 78

Figure 3-16. Retromer deletion strains respond to zinc.............................................................................. 79

Figure 3-17. Sensitivity of EtOH-vescalagin in zinc transporters deletion strains in vacuole and ER.

Figure 3-18. EtOH-vescalagin sensitivity of zinc transporters deletion strains to zinc chelator (TPEN) and zinc supplementation

Figure 3-19. EtOH-vescalagin increases intercellular zincosomes in WT and $\operatorname{zrt1} \Delta$ strains. ...... 83

Figure 3-20. Zinc and iron quantification by AAS method in WT and $z r t 1 \Delta$................................ 85

Figure 3-21. Ferrozin assay indicative of iron chelation activity of EtOH-vescalagin.................... 86 
Figure 3-22. Expression level of iron transporters in presence of EtOH-vescalagin and BPS..... 88 Figure 3-23. Quantification of GFP protein abundance of iron transporters in presence of EtOHvescalagin and BPS.

Figure 3-24. Expression level of zinc transporters in presence of $305 \mathrm{nM}$ EtOH-vescalagin and BPS.

Figure 3-25. Quantification of protein abundance of zinc transporters in presence of EtOHvescalagin and BPS 90

Figure 3-26. Mislocalization and expression level of retromer subunits in presence of EtOHvescalagin and BPS.

Figure 3-27. Quantification of GFP-protein abundance of retromer proteins in presence of EtOH-vescalagin and BPS

Figure 3-28. Retromer and zinc transporter expressions in iron deletion strains fet $3 \Delta$, fet $4 \Delta$, $s m f 3 \Delta$, fre $2 \Delta$ strains with different zinc and retromer GFP proteins.

Figure 3-29. Retromer and iron transporters expression in zinc deletion strains.

Figure 3-30. Iron and zinc transporters expression in retromer deletion strain. 100

Figure 3-31. Overexpression of iron, zinc transporters and retromer subunits regulates sensitivity to EtOH-vescalagin in WT 102

Figure 3-32. Overexpression of zinc transporters and retromer subunits in ftr1A strain 103

Figure 3-33. Overexpression of zinc transporters exacerbates sensitivity of fet4A to EtOHvescalagin . 103

Figure 3-34. Overexpression of retromer and iron transporters in zrt14 strain. 104

Figure 3-35. Overexpression of retromer and iron transporters in zrt2 $\triangle$ strain 105

Figure 3-36. Overexpression of retromer and iron transporters recues $z r c 1 A$ inhibition by EtOHvescalagin ..... 105

Figure 3-37. Overexpression of zinc and iron transporters in vps174 strain 106

Figure 3-38. Overexpression of zinc and iron transporters in vps294 strain. 107

Figure 3-39. Mitochondrial function is regulated by EtOH-vescalagin. 108

Figure 3-40 Model of EtOH-vescalagin mechanism of action in S. cerevisiae . ............................ 114

Figure 4-1. Established antifungal targets. ........................................................................ 118

Figure 4-2. Estragole structure. Drawn using ChemSpider........................................................... 119

Figure 4-3. Estragole dose response in WT BY4741 ........................................................... 122

Figure 4-4. ERG11 is not the primary target of estragole................................................................ 124

Figure 4-5. Genes in ergosterol pathway are not primary target of estragole................................ 125

Figure 4-6. Estragole does not mislocalize or change abundance of ergosterol in plasma membrane

Figure 4-7. GC-MS quantification of sterols in estragole- and ketoconazole-treated WT cells 127

Figure 4-8. Estragole mechanism is distinct from nystatin

Figure 4-9. FKS1 is not the primary target of estragole 
Figure 4-10. Estragole target is not LCB1.

Figure 4-11. CHS1 is not the primary target of estragole. ............................................................... 131

Figure 4-12. Estragole mechanism is distinct from flucytosine.................................................. 132

Figure 4-13. Combinatorial effect of estragole with known antifungals....................................... 134

Figure 4-14. Fitness plot of HET library in presence of estragole. ............................................... 136

Figure 4-15. Validation of residual growth of HET sensitive deletion strains in presence of estragole compared to WT strain. 137

Figure 4-16. Fitness plot of HOM library in presence of estragole

Figure 4-17. Validation of residual growth of sensitive mutants in presence of estragole compared to WT strain.

Figure 4-18. Validation of residual growth of 19 hits grew better in presence of estragole compared to WT strain. 142

Figure 4-19. Iron and Zinc exacerbate the inhibition by estrogole. 144

Figure 4-20. Glutathione rescues the estragole ROS level increase. 145

Figure 4-21. Estragole modifies the mitochondrial morphology 146

Figure 5-1. Preliminary data suggest anti-cancer activity of two feijoa compounds 157

Figure 5-2. Preliminary data suggests EtOH-vescalagin regulates glucose metabolism homeostasis 160

Figure 5-3. Preliminary data suggest EtOH-vescalagin regulates lipid droplets production..... 161 Figure 5-4. Preliminary data suggests EtOH-vescalagin regulates unfolded protein response 163 


\section{List of tables}

Table 1-1. List of $S$. cerevisiae libraries and their applications............................................................... 20

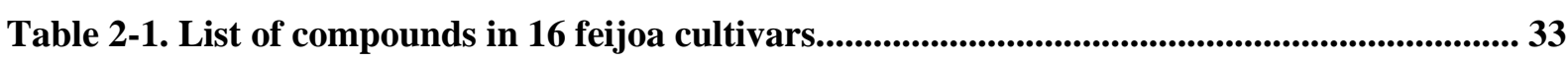

Table 2-2. Metabolites of feijoa extracts positively correlated with antifungal activity............... 41

Table 2-3. The antifungal activity of methanolic extracts $(0.01 \% \mathrm{v} / \mathrm{v})$ of 16 feijoa cultivars against saprobic $S$. cerevisiae and pathogenic Candida species.

Table 2-4. Half maximal inhibitory concentration $\left(\mathrm{IC}_{50}\right)$ of the authentic standards against saprobic $S$. cerevisiae and pathogenic Candida species......................................................................... 44

Table 3-1. Yeast strains used in this project 55

Table 3-2. Half maximal inhibitory concentration $\left(\mathrm{IC}_{50}\right)$ of EtOH-vescalagin in pathogenic Candida species. Values are from triplicate \pm standard deviation.

Table 3-3. Homozygous gene deletions sensitive and resistant to EtOH-vescalagin and involved functions.

Table 3-4. Heterozygous gene deletions sensitive to EtOH-vescalagin.

Table 3-5. Gene Ontology analysis of sensitive HET and HOM deletion strains to EtOHvescalagin.

Table 4-1. Half maximal inhibitory concentration $\left(\mathrm{IC}_{50}\right)$ of estragole in pathogenic Candida species.

Table 4-2. Annotation of sensitive HET deletion strains in presence of estragole.

Table 4-3. Annotation of sensitive deletion strains in presence of estragole in HOM library.... 141

Table 4-4. Annotation of deletion strains grew better in presence of estragole in HOM library. 


\section{Abbreviations:}

\begin{tabular}{ll}
\hline $\mathbf{Z n C l}_{2}$ & Zinc chloride \\
\hline FeCl $_{3}$ & ferric choloride \\
\hline FeCl $_{2}$ & Ferrous choloride \\
\hline EDTA & Ethylenediaminetetraacetic Acid \\
\hline DMSO & Dimethyl Sulfoxide \\
\hline EtOH-ves & Ethanol-adduct vescalagin \\
\hline GO & Gene Ontology \\
\hline ROS & Reactive oxygen species \\
\hline SC & Synthetic complete media \\
\hline GFP & Green fluorescent protein \\
\hline RFP & Red fluorescent protein \\
\hline SGD & Saccharomyces Genome Database \\
\hline BPS & Bathophenanthroline disulfonic acid \\
\hline FDR & False discovery rate \\
\hline HOP & Homozygous profiling \\
\hline HIP & Haploinsufficiency profiling \\
\hline MOA & Mechanism of action \\
\hline GCMS & Gas chromatography mass spectrometry \\
\hline WT & Wild type \\
\hline OCA & Synthetic genetic array \\
\hline & \\
\hline
\end{tabular}




\section{Chapter 1 Literature review}

\section{$1.1 \quad$ FUNGAL PATHOGENS}

Fungal pathogens are comprised of more than 150 different species representing the major phyla in the kingdom Fungi (Heitman 2011; Linton, et al. 2007). Immunocompromised people are particularly susceptible, such as several HIV and chemotherapy patients that suffer from fungal infections (San-Blas and Calderone 2008; Verduyn Lunel, et al. 2009). Unfortunately, mortality in fungal infected patients is approximately $35 \%$ as a consequence of treatment failure. Fungal treatments have been developed since the 1950s, and recently there has been an increase in the prevalence of fungal pathogens resistant to the currently available antifungal drugs (Pfaller 2012). Indeed, the cost of fungal infections in the US in 1998 was USD $\$ 2.6$ billion at the first year of infection, of which the vast majority (USD \$1.7 billion) was allocated to treat pathogenic species in the genus Candida (Wilson, et al. 2002). Invasive Candidiasis (IC), treatment by fluconazole in Australia was estimated about AU\$ 74,587 (USD\$55,192) per person and a bit less with anidulafungin with AU\$60,945 (USD\$45,87) (Grau, et al. 2015). More than $90 \%$ of fungal infections are caused by species in four different genera (Aspergillus, Candida, Cryptococcus and Pneumocystis) (Brown, et al. 2012).

There are three major types of fungal infections and these are distinguished by the mechanism of infection (Thiel 2010). Cutaneous infections are caused by species that enter the skin via wounds and these species can spread to new parts of the body (Thiel 2010). Microsporum and Trichophyton are the most common genera that cause the cutaneous infection type in humans and other mammals. The infection caused by these genera is not severe at first but can become serious in immunocompromised patients (Achterman and White 2011). Superficial infections usually occur in immunocompromised people (e.g., HIV patients) as well as healthy people exposed to the fungal pathogens and are caused by species 
that colonize the keratinized layer of skin or mucous membrane and can be also systemic if not diagnosed and treated quickly (Bondaryk, et al. 2013). For example, vaginitis and oral thrush are caused by Candida species with $C$. albicans the most common species. Systemic infections are caused by endemic mycoses or opportunistic fungi that penetrate part of the body beyond the site of infection, and the frequency of systemic infections has increased in recent years, primarily a consequence of resistance to antifungal drugs (Chakrabarti and Shivaprakash 2005). For example, Cryptococcus species are examples of endemic and environmental fungi that invade local sites in the body and subsequently cause life-threatening systemic infections in other body parts (Badiee and Hashemizadeh 2014). Hence, it is the immune system and antifungal drugs that must be effective in order to prevent systemic infections. The morbidity and mortality in this group of fungal infection is higher than others (Khan, et al. 2010).

\subsection{ANTIFUNGAL DRUGS}

As about 1.2 billion people suffer from fungal infections around the world (Denning and Bromley 2015), rapid diagnoses and effective antifungal drugs are critical to treating the infections. There are five classes of antifungals based on their molecular target (Campoy and Adrio 2017).

The first group targets the biosynthesis of ergosterol or ergosterol itself, the fungal equivalent of cholesterol that is a major component of the plasma membrane in fungi:

- Azoles: This is the largest class of antifungals commonly prescribed and prevent fungal growth by inhibiting the ERG11 gene product (14 $\alpha$-lanosterol demethylase), a key enzyme that converts lanosterol to ergosterol. Some examples are ketoconazole, fluconazole and miconazole that are prescribed for Candida and Cryptococcus neoformans infections (Campoy and Adrio 2017; Thompson, et al. 2009). 
- Polyenes: This group also disrupts the lipid bilayer in the plasma membrane but with a different mechanism of binding to ergosterol. Polyenes form pores in the plasma membrane (Zotchev 2003). These pores disrupt the membrane function resulting in cell leakage and cell death. Polyenes were the first clinically approved antifungal and have a wider spectrum of activity compared to all of other antifungals. Amphotericin B and nystatin are examples of polyenes (Hossain and Ghannoum 2000). Amphotericin B is used for the treatment of systemic infections such as Candida, Aspergillus and Cryptococcus (Laniado-Laborín and CabralesVargas 2009). Nystatin is used for oral candidiasis, vaginal candidiasis and also skin rashes, and is sometimes used in combination with other drugs (Blomgren, et al. 1998).

- Allylamines: This group of drugs inhibit the ERG1p squalene epoxidase and also interupt the ergosterol pathway, resulting in squalene accumulation, cell permeability, and cellular organization defects. Two major drugs of this group are terbinafine and naftifine, which are both fungistatic against Microsporum, Trichophyton and Candida species (Andriole 2000; Gupta, et al. 2008).

- Morpholines: These antifungals disrupt isomerase ERG2 and reductase ERG24, causing ergosterol depletion (Gupta, et al. 2008), and are used to treat fungal infections of toes and fingers. For example, the nail infection by Trichophyton mentagrophytes is better treated by morpholines than azoles (Campoy and Adrio 2017; Ghannoum, et al. 2013).

- The second group targets cell wall synthesis:

- B-Peptidoglycan synthesis inhibitors: Glucan comprises about $50 \%$ of the cell wall polysaccharide structure in fungi (Pfaller, et al. 2016). This group of antifungals are collectively referred to as echinocandins that inhibit the FKS1 protein integral to glucan synthesis. The echinocandins include caspofungin, micafungin and 
anidulafungin that are active against Candida and Aspergillus. These drugs are also used in combination with azoles to enhance their function and counter antifungal resistance (Pappas, et al. 2004).

- Chitin synthesis inhibitors: Chitin is another part of the cell wall that is linked to the glucan layer. As chitin is absent in human cells, chitin is an excellent target to treat fungal infections. Nikkomycin and polyoxine are two major drugs from this group and are able to cure dimorphic fungal and phytopathogenic infections (Mehta, et al. 1984; Ruiz-Herrera and San-Blas 2003).

- The third group of antifungal drugs target sphingolipids:

- Sphingolipid biosynthesis inhibitors: As sphingolipids are a part of the lipid layer in all cell membranes, sphingolipid inhibition results in structural and signaling defects in the cell membrane. Aureobasidin A is an antifungal from this group used to treat Schizosaccharomyces, Saccharomyces, Candida and Aspergillus infections.

- The fourth group of antifungal drugs target nucleic acids:

- Nucleic acid synthesis inhibitors: Cells are non-viable without the synthesis of DNA and RNA. 5-Flucytosine is the main drug from this group that acts after entering the cell via cytosine permease and incorporates 5-fluorouracil leading to interruption of the synthesis of DNA and RNA (Vermes, et al. 2000). It is active against Candidiasis, Cryptococcosis and Chromoblastomycosis and is sometimes prescribed in combination with azoles or polyenes (Campoy and Adrio 2017; Vermes, et al. 2000).

- The fifth group of antifungal drugs targets proteins:

- Protein synthesis inhibitors: This group of drugs disrupt enzymatic activity that consequently inhibits protein synthesis. Tavaborole is approved to treat infections caused by Trichophyton rubrum and also fungi (Gupta and Versteeg 2016). 
Cispentacine and sordarine are other antifungals in this group used to treat infections caused by Candida albicans (Khan and Jain 2000).

The number of known antibacterial drugs is higher than antifungals as fungi are eukaryotes that share some candidate targets with mammalian cells. Thus there is a need to be more cautious about choosing the right mechanism underlying their function, ideally including a target that does not exist in humans (Campoy and Adrio 2017). Moreover, different antifungal sources need to be studied to improve chances of finding a new class of bioactive (Eliopoulos, et al. 2002).

\subsection{ANTIFUNGAL DRUG RESISTANCE}

One of the main reasons of the high mortality and morbidity from fungal diseases is drug resistance (Giedraitiene, et al. 2011; Kanafani and Perfect 2008). Drug resistance is defined as a nonsusceptibility of the microorganism in which the MIC (minimum inhibitory concentration) of the drug exceeds the susceptibility breakpoint. It can happen via different mechanisms and can also be a primary or secondary acquired trait. Since 1970, the rate of death by Candidiasis has increased significantly and more efforts have begun to find new therapeutic antifungals.

Firstly, amphotericin B has been used as the major antifungal followed by azole drugs, of which resistance has been developed against both of the drugs. Resistance of Candida species to triazoles was reported in 2002 in the US. For C. albicans, secondary resistance typically occurs in $25 \%$ of HIV-infected or chemotherapy patients who are getting prolonged treatment with azoles (Eliopoulos, et al. 2002). Secondary resistance to flucytosine was also reported when it is used as a monotherapy which led to its use with other drugs (Vanden, et al. 1997).

Antifungal resistance can evolve through alteration of the drug target enzyme like cytochrome P-450 lanosterol $14 \alpha$ demethylase synthase enzyme in azole resistance 
(Eliopoulos, et al. 2002; Lopez-Ribot, et al. 1998). There are also several reports of amphotericin B resistance of $C$. lusitaniae and $C$. guilliermondii as a primary resistance (White, et al. 1998). Aspergillus species have also evolved resistance to azoles and sometimes cross resistance between two drugs is also seen. Resistance to flucytosine and fluconazole has also evolved in treatments of Cryptococcus meningitis as a secondary resistance in HIV patients (Assing, et al. 2003; Whelan 1987).

Finally, the huge usage of these drugs that has resulted in the development of resistance has resulted in the considerable need for new antifungal drugs (Arif, et al. 2009). Several efforts have begun to discover new medicines with new mechanisms of action, including investigating synthetic drugs and natural products (Arif, et al. 2009; Giedraitiene, et al. 2011; Kanafani and Perfect 2008).

\subsection{NATURAL PRODUCTS AND DRUG DISCOVERY}

Natural products are small chemical entities with a variety of biological functions in medicine and agriculture, and sourced from biological organisms such as bacteria, plants, fungi etc (Katz and Baltz 2016). For example, secondary metabolites (natural products) produced by an organism are beneficial but not required for survival of the organism (Katz and Baltz 2016). Natural products have been used from ancient times for treating illnesses such as extracts of the cypress tree Cupressus sempervirens for treating coughs (Dias, et al. 2012) or the Scots lovage Ligusticum scoticum to treat scurvy (Ray 1724).

Overall, drug discovery is complex and requires significant investments of time, research and money (Katz and Baltz 2016; Kubinyi 2006). Only one of every 5-10,000 active molecules gets approved to be used in the clinic (Khosla and Keasling 2003; Kubinyi 2006). Given this success rate and the fact that nature was the source of nearly all medicines historically, pharmaceutical companies have exploited natural products working off the premise that these compounds have a function in nature that is potentially useful to manipulate 
in human medicine. From 1931-2010, more than half of the approved drugs were natural products or based on natural products (Katz and Baltz 2016). For example, more than half of the approved anticancer drugs from 1940-2014 were natural products or based on natural products (Newman and Cragg 2016) (Figure 1.1). More recently, approximately one-third of small molecule medicines approved between 1982-2010 were derived from nature (Harvey, et al. 2015).

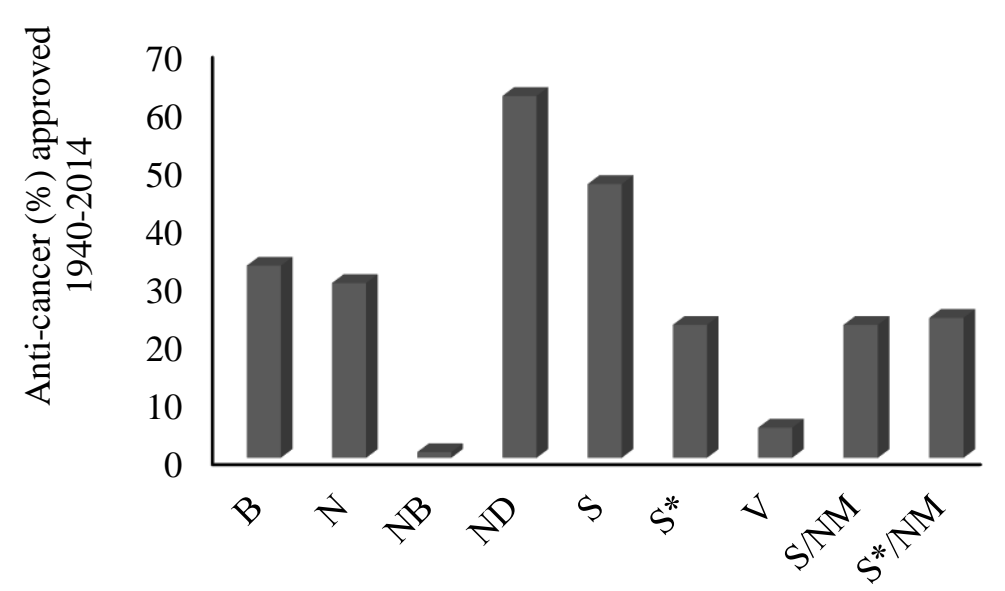

Figure 1-1. Anti-cancer drugs approved between 1940 and 2014, B) biological macromolecules, N) unaltered natural product, NB) botanical drug, ND) natural product derivatives, S) synthetic drug, $\mathrm{S}^{*}$ ) Synthetic drug NP, V) vaccine, NM) mimic of natural product. Data adopted from Newman et al., 2016.

\subsubsection{Plant-derived bioactive compounds}

Plants are rich sources of structurally and functionally diverse bioactive secondary metabolites such as tannins, terpenoids, alkaloids, and flavonoids (de Souza, et al. 2008). For example, both morphine and aspirin are historically important plant-derived medicines isolated from Papaver somniferum and Salix alba, respectively (Butler 2004b; Dias, et al. 2012). Plantderived compounds can play a range of roles in several processes such as anticancer, antiinflammatory, antibacterial and antifungal processes (Amaral, et al. 2009; Barbosa Pelegrini, 
et al. 2011; Butler 2004a; Cowan 1999; Satish, et al. 2007). It has been reported since the 1940s that approximately $60 \%$ of anticancer drugs and $75 \%$ of antibacterial drugs are from natural products or their derivatives (McChesney, et al. 2007). Taxol, the first approved anticancer drug was isolated from fungal endophytes of Pacific yew tree (Taxus brevifolia) in the 1970's and approved by FDA in 1992 and this approach paved the way for additional discoveries of plant-derived anticancer compounds (Balandrin, et al. 1993; Gangadevi and Muthumary 2008). A compound that has been pivotal in fighting malaria is artemisinin, which was derived from Artemisia annua in 1971 (Balandrin, et al. 1993).

Despite the prominence of prescribed medicines being either isolated from plants or based on plant-derived compounds (Yun, et al. 2012), there are some challenges to working with plant metabolites such as complex structures that make it difficult to synthesize compounds (Yun, et al. 2012) and it is often not cost effective (Atanasov, et al. 2015). Therefore, scientists have continued to develop new platform technologies to overcome these issues (Yun, et al. 2012). In the cases of taxol and artemisinin, the synthesis has been optimized to include genetically modified organisms as sources of advanced precursors, which overall maximized production without a negative impact to native plant populations (DeJong, et al. 2006; Zeng, et al. 2008).

Several metabolites from plants exhibit antifungal bioactivity (Arif, et al. 2009). These secondary metabolites include polyphenols, flavonoids, coumarins, quinones, saponins, xanthones, terpenoids, alkaloids, lectins, and polypeptides. Crude extracts of some plants have been investigated for antifungal activity, but not many have been studied for the active components. For example, extracts of Rosmarinus officinalis and Nigella sativa were reported with antifungal activity against $C$. albicans without any further investigation into identifying the antifungal compounds in the extracts (Amrouche, et al. 2011; Dalleau, et al. 2007; Singh, et al. 2005). In some cases, the antifungal activity has been commercialized such as Kolorex, 
an antifungal agent derived from the mountain pepper tree Pseudowintera colorata (horopito) in New Zealand, yet the specific antifungal compound was not known until 2000 when it was identified as polygodial (Berry-Kilgour 2002).

\subsubsection{Microbial-derived bioactive compounds}

Arguably the most prominent compound derived from microbes is penicillin, an antibacterial compound isolated from the filamentous fungus Penicillium notatum (Fleming 1929). Penicillin was discovered in 1928 and continues to be prescribed today to treat bacterial infections, although many types of bacteria have developed resistance to penicillin (Pallares, et al. 1995). Sirolimus, also known as rapamycin, is another commonly prescribed antibiotic that was originally isolated from a soil sample of bacterium Streptomyces hygroscopicus (Seto 2012).

Several antifungal compounds also have been isolated from microbial sources in nature (Vicente, et al. 2003). Amphotericin B was isolated from Streptomyces nodosus in 1995 as an antifungal compound that disrupts the normal function of ergosterol in the fungal plasma membrane (Caffrey, et al. 2001). Polyoxin and nikkomycin were antifungal via the inhibition of chitin synthesis in the fungal cell wall and both were isolated from different species of Streptomyces bacteria (Vicente, et al. 2003). Pradimicins A and B are macrolactam antifungal drugs isolated from bacterial Actinomadura species with remarkable antifungal activity against Candida and Cryptococcus species (Oki, et al. 1990). Hypoxydordarin was isolated from Hypoxylon croceum and shown to exhibit a wide range of activity against yeast and filamentous fungi (Daferner, et al. 1999). Echinocandins are antifungal compounds isolated from the fungus Glarea lozoyensis that are antifungal via disrupting fungal cell wall synthesis (Schwartz, et al. 1992). 


\subsubsection{Marine organism-derived bioactives}

Because of the extensive biodiversity in the world's oceans, the sea is one of the richest sources of bioactive compounds (Hamed, et al. 2015). With extensive species diversity, these compounds are equally diverse with multiple biological functions from compounds such as peptides derived from fish and alkaloids isolated from sea squirts (Lordan, et al. 2011). Fish oils are a rich source of anti-cardiovascular omega-3 fatty acids (Weitz, et al. 2010). Approximately 30,000 species of red, green and brown algae are a source of polyphenolic antioxidants with antibacterial and antifungal bioactive components (Bhakuni and Rawat 2006; Onofrejová, et al. 2010; Plaza, et al. 2010; Sheih, et al. 2009).

The marine organisms including marine algae and microorganisms also exert antifungal activity (Xu, et al. 2015). The antifungal forazoline A was isolated from marine invertebrate-associated bacteria and was shown to be potently antifungal against $C$. albicans via disruptions of the fungal plasma membrane (Wyche, et al. 2014). The quinazolinone alkaloids were derived from marine Bacillus cereus and shown to be active against $C$. albicans (Xu, et al. 2011b).

\subsection{FEIJOA FRUIT AND BIOACTIVITY}

Feijoa (Acca sellowiana) is a monotypic species in the Myrtaceae family (Weston 2010). The feijoa fruit also commonly known as the pineapple guava, has a smooth green skin and soft, off-white flesh, which ripens on the tree to the size of a small apple (Figure. 1.2). The pulp is sweet and aromatic. Feijoa fruit, like the guava, is a source of vitamin C, low in calories, and high in minerals (potassium, phosphorus and iodine) (Watson and Preedy 2010) and fiber. In addition to consumption of the whole pulp, the pulp is used as an ingredient in ice cream, smoothies, wine, yogurt, juice, chutney and jam. Originating in South America, feijoa was introduced to New Zealand in the 1920's and currently is a NZD\$1.7 million crop (Saunders, 
et al. 2011) with fruits available from March through June. Approximately 500 feijoa growers exist in New Zealand, of which five growers export 25-54 tons annually, primarily to Australia and other countries as well (Saunders, et al. 2011).
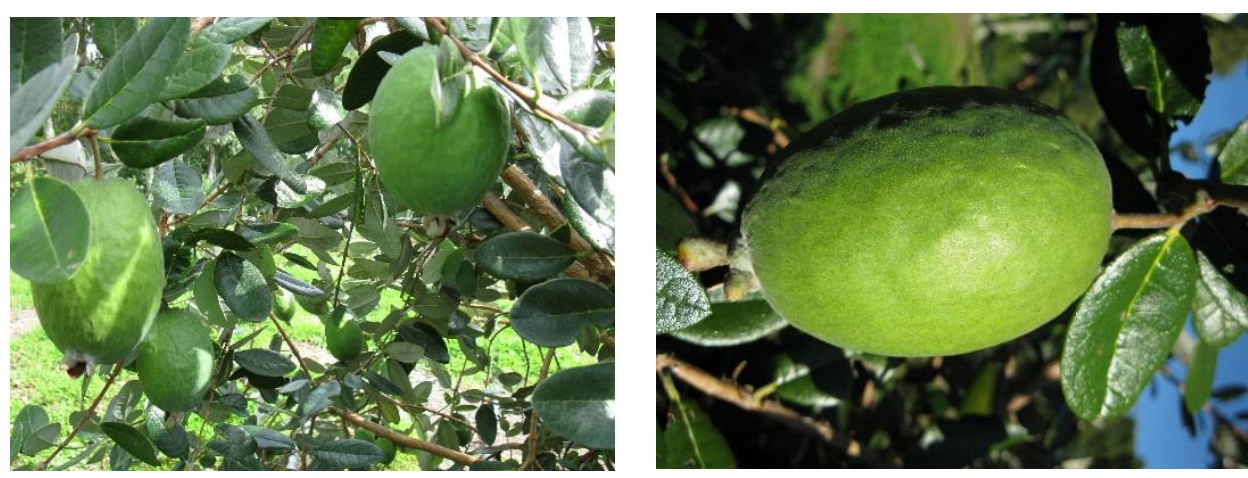

Figure 1-2. Feijoa fruit (Acca sellowiana). Images provided by Foretaste Feijoa Fruit Ltd., Takaka, NZ.

Feijoa has been reported to contain several bioactive compounds providing different functions (Weston 2010). Generally, one main group of bioactive compounds are the essential oils including hydrophobic volatile aroma compounds. These compounds have diverse yet integral functions in signaling to pollinators and protecting plants against ultraviolet light, herbivores, and pathogens (Fornari, et al. 2012; Prabuseenivasan, et al. 2006).

Consistent with other plants, feijoas are also rich in aromatic volatiles (Watson and Preedy 2010). The amounts and types of the volatiles are influenced by the different parts of the fruit and other conditions. Based on a study in New Zealand, the major oils in feijoa peels are (Z)-hex-3-en-1-ol (20\%), linalool (18\%), methyl benzoate (14.5\%), germacrene D (6\%) and octan-3-one (6\%) (Shaw, et al. 1989). This was not consistent with a study in France that identified different compounds as the major oils, specifically $\beta$-caryophyllene (12\%), ledene (9.5\%), $\alpha$-humulene (6.5\%), $\beta$-elemene (5\%), d-cadinene (5\%) and bicyclogermacrene $(4.5 \%)$ (Fernandez, et al. 2004; Shaw, et al. 1989; Shaw, et al. 1990) while the volatiles in Japanese feijoa were consistent with New Zealand sourced fruit (Weston 2010). The other groups of 
feijoa bioactive compounds include alkaloids, polyphenols, and flavonoids that confer different functional activities.

Antibacterial activity: An acetonic extract of feijoa fruit exhibited antibacterial activity against pathogenic Helicobacter pylori (Basile, et al. 2010). It has been reported in the same study that flavone was the most active compound in the fruit. In a separate study, feijoa seeds had the highest antibacterial activity against eight pathogenic bacterial species compared to other parts of the feijoa fruit (Basile, et al. 1997). Likewise, the aqueous extract of feijoa leaves conferred antibacterial activity against Pseudomonas aeruginosa and Enterobacter species (Vuotto, et al. 2000) as did the essential oils and methanolic extract of feijoa peels (Nakashima 2001; Saj, et al. 2008; Varga and Molnar 2000).

Anti-inflammatory activity: It has been reported that feijoa has an anti-inflammatory effect as it suppresses nitric oxide production by flavone (Rossi, et al. 2007). A recent study on anti-inflammatory effects in 10 fruits demonstrated that the strongest anti-inflammatory effect was allocated to feijoa, and this activity was via a mechanism that included pathways involving the Toll-like receptor (TLR2) family. These results represent the potential of exploiting this fruit to develop some complementary therapies to treat inflammation (Nasef, et al. 2015).

Anti-cancer activity: Acetonic extracts of feijoa fruits exhibited anti-cancer activity on cultured cancer cells through the induction of apoptosis (Bontempo, et al. 2007). Feijoa peel extract also showed anti-tumor activity against human oral cancer cell lines (HSG-2 and HSG); this activity was limited to two fractions of the peel extract, one of the fractions belonged to the acetonic extract and the other one was from methanolic extraction (Varga and Molnar 2000).

Antifungal activity: Feijoa acetonic extracts have demonstrated antifungal activity against human (C. albicans) and plant (Botrytis cinerea and Ralstonia solani) pathogens (Basile, et al. 2010). Methanolic extracts of feijoa peel have also exhibited antifungal activity 
against Candida species (Varga and Molnar 2000). In addition, feijoa essential oils were also antifungal against C. albicans and Aspergillus niger (Saj, et al. 2008).

Overall, there have been 18 studies of feijoa bioactivity (Figure 1.3). Antibacterial activity has been most studied (36\% of total studies) while anti-cancer and anti-inflammatory activity have been explored in $16 \%$ of the studies. Only $11 \%$ of the studies investigated antifungal activity. These data show there is a need to study feijoa fruit compounds as potential sources of new antifungal drugs.

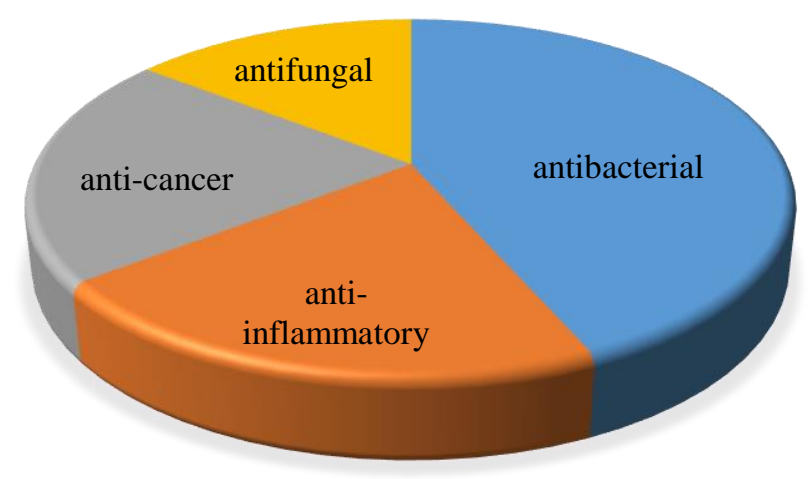

Figure 1-3. Distribution of studies investigating four types of bioactivity in feijoa.

\subsection{METABOLITE PROFILING}

Different analytical methods to measure metabolite composition in a sample have been well established whereby each method aims to generate a metabolite profile or metabolic fingerprint (Fernie, et al. 2004). Liquid Chromatography (LC) or Gas Chromatography (GC) coupled with Mass Spectrometry (MS) are two common methods to identify and quantify hundreds of metabolites in extracts. The metabolites identified will be dependent on the method used to generate the extract as well as the chromatography (LC or GC). For all extracts and chromatography, metabolite identification can be performed by comparison with a database of mass spectrometric profiles for known compounds (e.g., the National Institutes of Standards 
and Technology (NIST) database for GC-MS profiles) (Lemmon, et al. 2015) although confident identification then requires comparison with an authentic standard or further purification and spectrometry.

One approach to analyse metabolite profiles is multivariate analysis (Worley and Powers 2013). Multivariate analysis methods based on statistics have been used to describe complex data relationships when there is more than one variable. There are many statistical methods to perform multivariate analysis with principal components analyses and partial least square analysis as the two most common approaches (Ohno, et al. 2011; Wold, et al. 2001). These analyses identify relationships between metabolites within a large metabolite dataset. For example, studies of cotton plants to assess the effect of silicon on antioxidant enzymes used multivariate analysis to identify the pattern describing silicon's influence on lipid peroxidation (Alberto Moldes, et al. 2013). Multivariate analysis has been used to correlate crop cultivars and bioactive molecules. For example, GC-MS, LC-MS and NMR data have been used with multivariate analysis to find correlations between different cultivars of Glycyrrhiza glabra and their bioactive metabolites (Farag, et al. 2012).

\subsection{CHEMICAL GENETICS}

\subsubsection{Yeast as a model organism for bioactivity and chemical genetics}

In the event that the bioactivity and/or mechanism of action of an extract and/or compound is not known, chemical genetics is a reliable method to elucidate genes and phenotypes regulated by chemicals (Parsons, et al. 2006). Chemical genetics is thus an extension of classical genetics used to investigate genes and phenotypes. As chemical genetics aims to relate genes and chemicals, it is a requirement to conduct chemical genetic analyses in model organisms for which extensive genetic resources are available. Genetic mechanisms of 
chemical compounds have been determined using yeast by elucidating the relationship between genotype and phenotype (Kubinyi 2006). Saccharomyces cerevisiae (Baker's yeast) was the first eukaryotic organism with its genome sequenced in 1996, which has resulted in a powerful resource of genetic reagents (e.g., haploid and diploid genomic libraries of deleted, overexpressed and GFP-tagged genes). Yeast is a powerful genetic model due to these libraries as well as being unicellular with a small physical size, a compact genome of $\sim 6,000$ genes, a rapid 90-minute generation time, efficient homologous recombination, and high homology to the human genome (Botstein and Fink 2011; Foury 1997). In particular, chemical genetic profiling takes advantage of these genome-wide libraries that modulate gene function to probe the cellular roles of bioactive compounds (Simon and Bedalov 2004) and it is based on the logic that the primary effect of the extract and/or compound is binding to the gene product and interrupting its function (Parsons, et al. 2006) (Figure 1.4). For example, the anticancer compound (peloruside A) was isolated from a marine sponge (Mycale hentscheli) (Hood et al. 2002) and chemical genetics in yeast was used to confirm the compound functioned by stabilizing microtubules (Best, et al. 2013). 


\subsubsection{Non-essential gene deletion libraries}

There are about $\sim 4,800$ non-essential genes in yeast. In the non-essential deletion library, each gene has been deleted and replaced by the antibiotic resistance marker (KanR) that confers resistance to the antibiotic kanamycin (similar to geneticin, G418). The antibiotic resistance gene is flanked by $20 \mathrm{bp}$ barcodes upstream and downstream that are unique for each gene deletion (Boone, et al. 2007; Winzeler, et al. 1999). The non-essential gene deletion library exists in the haploid background in either mating type (MATa or MAT $\alpha$ ) as well as the diploid background as homozygous or heterozygous mutants. Non-essential deletion mutants can be investigated in a HOP (homozygous profiling) assay of homozygous diploid mutants as well as a HIP (heterozygous profiling) assay of heterozygous diploid mutants (Table 1.1).

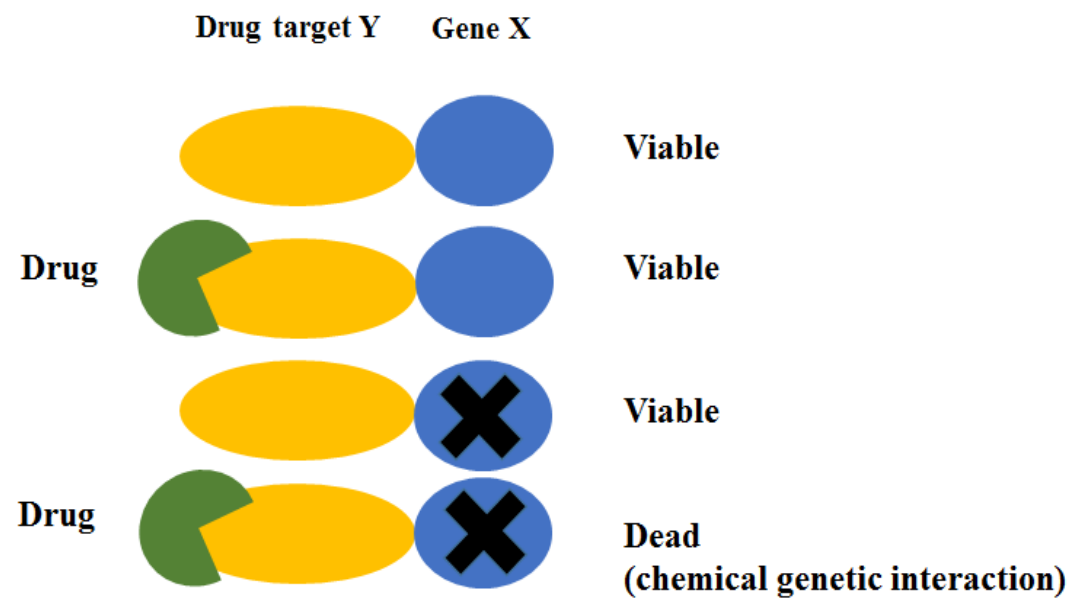

Figure 1-4. An overview of a chemical genetic interaction. When a drug is targeting a pathway that is driven by two genes, and one gene is deleted and another inhibited by the drug, the cell cannot survive. 
The chemical genetic profile can be accomplished via quantification of growth of individual strains in the presence and absence of the extract/compound on agar (Boone, et al. 2007; Parsons, et al. 2004). Briefly, the growth of the non-essential deletion library can be quantified on agar media in the presence and absence of the extract/compound. Reduced growth in the extract/compound treatment compared to control media implicates that gene and its associated pathway in the metabolism of the extract/compound (Figure 1.4). Chemical genetic analyses in agar media was done for 82 compounds, from which the hypersensitive mutants were used to provide insight to the main pathways targeted by most of the test compounds (Parsons, et al. 2006). Alternatively, the chemical genetic profile of this library can be accomplished via quantification of barcodes in a competitive growth assay wherein all strains are grown in the presence and absence of the extract/compound in a small volume (1$5 \mathrm{~mL}$ ) of liquid (Ammar, et al. 2009; Robinson, et al. 2014; Smith, et al. 2010). Rather than measuring growth of individual strains in the agar assay, the competitive growth assay measures growth by barcode abundance. Reduced barcode abundance for a particular deletion indicates that the strain was sensitive to the compound, uncovering the genes mediating the main mechanism of the compound. This approach was recently used to predict mechanism of action of 3,250 compounds (Lee, et al. 2014). Quite powerfully, this competitive growth assay was conducted on diploid mutant libraries whereby the HIP assay identified the primary target of compounds and the HOP assay identified genes and pathways buffering the mechanism of action of the compounds.

\subsubsection{Essential gene deletion libraries}

The yeast genome contains about 1,200 essential genes (about 19\% of the genome) that are required for survival. Essential gene knockdown libraries are available to investigate 
functions of essential genes. There are four major essential gene knockdown libraries. First, the temperature-sensitive library was constructed via the integration of a temperature-sensitive promoter upstream of essential genes resulting in knockdown that is dependent on incubation temperature (Ben-Aroya, et al. 2010; Li, et al. 2011). Second, the tet-off library was constructed via the integration of a tetracycline promoter upstream of essential genes resulting in knockdown that is titratable by the amount of doxycycline in the growth media (Mnaimneh, et al. 2004). Third, the DAMP (Decreased Abundance of mRNA Perturbation) library was constructed via the integration of a kanamycin-resistance cassette in the 3'-UTR of essential genes resulting in knockdown that can be selected for in growth media containing kanamycin (Breslow, et al. 2008). Lastly, the essential genes can be investigated in heterozygous diploid mutants (Lee, et al. 2014). The DAMP collection has been used to identify the drug mechanisms in chemical genetic profiling for nine known drugs, including tunicamycin, fluconazole and miconazole and could successfully identify drug targets like ERG11 as the target of fluconazole (Yan, et al. 2008).

\subsubsection{Overexpression libraries}

Both essential and non-essential genes can be investigated with two overexpression libraries in which the genes are overexpressed using a galactose-inducible promoter. The first overexpression library consists of 5,280 yeast strains in which the galactose-inducible promoter is upstream of the gene containing a GST-tagged N-terminus (Sopko, et al. 2006). The second overexpression library consists of 5,800 plasmids in which the galactose-inducible promoter is upstream of the gene (Zhu, et al. 2001). Growth of strains can be compared on glucose (repressed expression) and galactose (induced overexpression). Yeast overexpression libraries have been used to identify candidate drugs to treat neurodegenerative diseases (Khurana, et al. 2015). 


\subsubsection{GFP library}

A major goal of molecular biology is to characterize the functions of all proteins in the cell (Huh, et al. 2003) (Tkach, et al. 2012). The yeast green fluorescent protein (GFP) library includes about 4,150 strains wherein each strain has the GFP protein tagged to the C-terminus of the wild-type version of distinct proteins (Huh, et al. 2003) (Table 1.1). A major advantage of the GFP library is that fluorescence can be observed in living cells, enabling scientists to directly view protein localization and abundance in a systematic library of strains representing $75 \%$ of the proteome (Huh, et al. 2003). The GFP emits a bright green light at $488 \mathrm{~nm}$ visualised under the fluorescent microscope which can be seen in different cellular organelles based on the function of that protein (Tkach, et al. 2012). For example, high-throughput image analysis of the GFP library identified the genes and pathways critical to the mechanisms of action of two established DNA damaging agents (Tkach, et al. 2012). In addition, the GFP library can be used in the background of the gene deletion library. For example, genes required for the secretory pathway were identified via analyses of the Mrh1-GFP as a marker for the secretory pathway (Bircham et al 2011). 
Table 1-1. List of S. cerevisiae libraries and their applications.

\begin{tabular}{|c|c|c|c|c|}
\hline yeast collection & phenotype & genes to study & $\begin{array}{l}\text { number of } \\
\text { genes }\end{array}$ & reference \\
\hline $\begin{array}{l}\text { Heterozygote diploid } \\
\text { collection }\end{array}$ & $\begin{array}{l}\text { deleted one gene } \\
\text { copy }\end{array}$ & $\begin{array}{l}\text { non/essential } \\
\text { genes }\end{array}$ & 6000 & Lee et al., 2014 \\
\hline $\begin{array}{l}\text { Homozygote diploid } \\
\text { collection }\end{array}$ & $\begin{array}{l}\text { deleted two gene } \\
\text { copies }\end{array}$ & $\begin{array}{l}\text { non-essential } \\
\text { genes }\end{array}$ & 6000 & Lee et al., 2014 \\
\hline Haploid collection & deleted whole gene & $\begin{array}{l}\text { haploid non- } \\
\text { essential genes }\end{array}$ & 6000 & $\begin{array}{l}\text { Parson et al., } \\
2006\end{array}$ \\
\hline GFP-collection & $\begin{array}{l}\text { fluorescent tagged- } \\
\text { proteins }\end{array}$ & $\begin{array}{l}\text { non-essential gene } \\
\text { proteins }\end{array}$ & 4159 & $\begin{array}{l}\text { Huh et al., } \\
2003\end{array}$ \\
\hline DAMP collection & $\begin{array}{l}\text { decreased gene } \\
\text { expression }\end{array}$ & essential genes & 800 & $\begin{array}{l}\text { Breslow et al., } \\
2008\end{array}$ \\
\hline Tet collection & $\begin{array}{l}\text { decreased gene } \\
\text { expression }\end{array}$ & essential genes & 800 & $\begin{array}{l}\text { Mnaimneh et } \\
\text { al., } 2004\end{array}$ \\
\hline $\begin{array}{l}\text { Temperature sensitive } \\
\text { collection }\end{array}$ & $\begin{array}{l}\text { decreased gene } \\
\text { expression }\end{array}$ & essential genes & 787 & Li et al., 2011 \\
\hline Overexpression collection & $\begin{array}{l}\text { increased gene } \\
\text { expression }\end{array}$ & $\begin{array}{l}\text { over dosage of all } \\
\text { genes }\end{array}$ & 5800 & Zhu et al., 2001 \\
\hline GST-tagged collection & $\begin{array}{l}\text { increased gene } \\
\text { expression }\end{array}$ & $\begin{array}{l}\text { over dosage of all } \\
\text { genes }\end{array}$ & 5280 & $\begin{array}{l}\text { Sopko et al., } \\
2006\end{array}$ \\
\hline
\end{tabular}

\subsubsection{Synthetic Genetic Array Analysis}

\subsubsection{Gene-gene and gene-gene-drug epistasis}

The haploid deletion library has also been powerful in elucidating gene-gene interactions (Tong, et al. 2001). The main method used to investigate gene-gene interactions is the Synthetic Genetic Array (SGA), which in essence is a cross between a query deletion mutant with the 4,800 strains in the deletion library that ultimately results in the generation 
of a haploid, genome-wide representation of the query deletion strain with an additional deletion (Tong, et al. 2001). Growth of the double deletion is compared against the single deletions, and a synthetic lethal interaction is identified if the double deletion was not viable when the single deletions were. Likewise, a synthetic sick interaction is identified if the double deletion exhibits a growth defect relative to the single deletions. Observations of synthetic lethality and synthetic sick results indicate a genetic interaction between the two genes, which lead to elucidating whether the interacting genes are in the same or compensating pathways (Giaever and Nislow 2014; Tong, et al. 2004). Growth of single and double mutants can also be quantified in the presence and absence of a drug, thus providing insight into gene-gene-drug interactions. Extensive numbers of SGA analyses have been conducted (Bender and Pringle 1991; Montpetit, et al. 2005; Ooi, et al. 2003; Parsons, et al. 2004; Yan Tong and Boone 2006), including SGA analyses starting with 6000 query genes that identified nearly 1 million gene-gene interactions (Costanzo, et al. 2016). A relatively small subset of SGA analyses examined double deletions with the addition of a drug, albeit it was highly informative for those that did. For example, ERG11 deletion strain was crossed with the whole library and 27 double mutants were synthetic lethal or synthetic sick only in the presence of fluconazole, thus providing additional insight into the mechanism of fluconazole (Parsons, et al. 2004).

\subsubsection{Gene-protein epistasis}

Interactions between genes and proteins can be investigated by screening the GFP library with gene deletions in the background (Sassi, et al. 2009). This can be accomplished using a modification of the SGA methodology to introduce a gene deletion in the background of the GFP library, which results in the measurement of protein abundance and localization in the context of specific gene deletions. For example, the Cps1 protein is sorted in multivesicular bodies and then accumulates in the vacuole lumen in WT or in endosomes in 
ESCRT (endosomal sorting complex required for transport ) deletion strains such as vps $23 \Delta$ (Chu, et al. 2006).

\subsection{AIMS AND HYPOTHESIS}

The main question of this project is whether feijoa fruit has any therapeutic potential as a source of a novel antifungal drug, and if yes, which compounds and mechanisms mediate the antifungal activity. I hypothesized that identifying compounds in novel feijoa cultivars would improve our understanding of the chemical diversity of antifungal compounds in feijoa and determining the antifungal mechanism of action of feijoa compounds would provide insight into the pharmaceutical potential of these compounds. Specifically, my aims were the following:

Aim 1 (Chapter 2): To evaluate the antifungal compounds in feijoa. Here I used metabolite profiling of feijoa cultivars to identify the most potent antifungal compounds, of which the most potent was 4-cyclopentene-1,3-dione for which little is known about antifungal activity.

Aim 2 (Chapter 3): To identify the antifungal mechanism of action of an ethanol adduct of vescalagin that was isolated for the first time from feijoa fruit. Here I used an array of chemical genetic analyses to determine that this compound is antifungal via iron chelation, zinc toxicity, and disrupted retromer recycling.

Aim 3 (Chapter 4): To identify the antifungal mechanism of action of estragole that had previously been isolated from feijoa fruit. Here I used chemical genetic analyses to determine that this compound is antifungal via a mechanism distinct from known antifungals. 


\section{Chapter 2 Bioactivity-Guided Metabolite Profiling of Feijoa Cultivars}

\subsection{INTRODUCTION}

Nature has been a rich source for pharmaceutical compounds, underlying $80 \%$ of our currently prescribed drugs (Cragg and Newman 2013). In particular, many noteworthy drugs in the history of human civilization have been derived from plants (e.g., morphine and aspirin to treat pain and headaches, artemisinin to treat malaria). Just as there is a potential epidemic due to bacteria developing resistance to current antibiotics and delays in the development of new anitbiotics (Crofts, et al. 2017), the same applies to pathogenic fungi. In recent years, the limited nature of our current arsenal of antifungal drugs has been manifest in the resistance of pathogenic fungi to current antifungal drugs, increases in rates of fungal diseases in immunocompromised patients (e.g., chemotherapy, transplant, and HIV patients), and increases in the reports of new pathogenic species (Perfect 2017). Like antibiotics, most antifungal drugs have historically been based on natural products isolated from, or derived from cultured microbes. However, given that plants are consistently regulating infections by fungi (i.e., permitting infection by mutualistic fungi and resisting infection by pathogenic fungi), extracts of plants including fruits are also a promising source of novel antifungal compounds (RazzaghiAbyaneh and Rai 2013).

Feijoa (Acca sellowiana, Myrtacaeae) is native to Southern Brazil, Northern Argentina and Western Paraguay, where it is also known as pineapple guava. It is actively cultivated as an annual multi-million dollar (>\$8 USD) agricultural crop in temperate and subtropical climates in the United States of America, South America, Europe, Australia and New Zealand. The pulp of the fruit (Figure. 2.1) is consumed in its entirety or as a juice, as well as in food products such as ice cream, chutney, chocolate, and wine. The fruit pulp has a gritty texture with a distinctive highly perfumed odor and a flavor that is is sweet to mid-sour. Bioactive compounds with anticancer, antioxidant, antiviral, anti-inflammatory, anti-diabetic, probiotic 
and antibacterial activities have been isolated from the leaves, pulp and peels of commercial cultivars of the thick-skinned feijoa fruit (Hap and Gutierrez 2012; Martin, et al. 2015; Nasef, et al. 2015; Turco, et al. 2016; Weston 2010). However, antifungal compounds in feijoa are dramatically understudied with only four reports to date (Basile, et al. 2010; GD, et al. 2016; Nakashima 2001; Saj, et al. 2008), none of which has either identified mechanism of action or the bioactive compound.
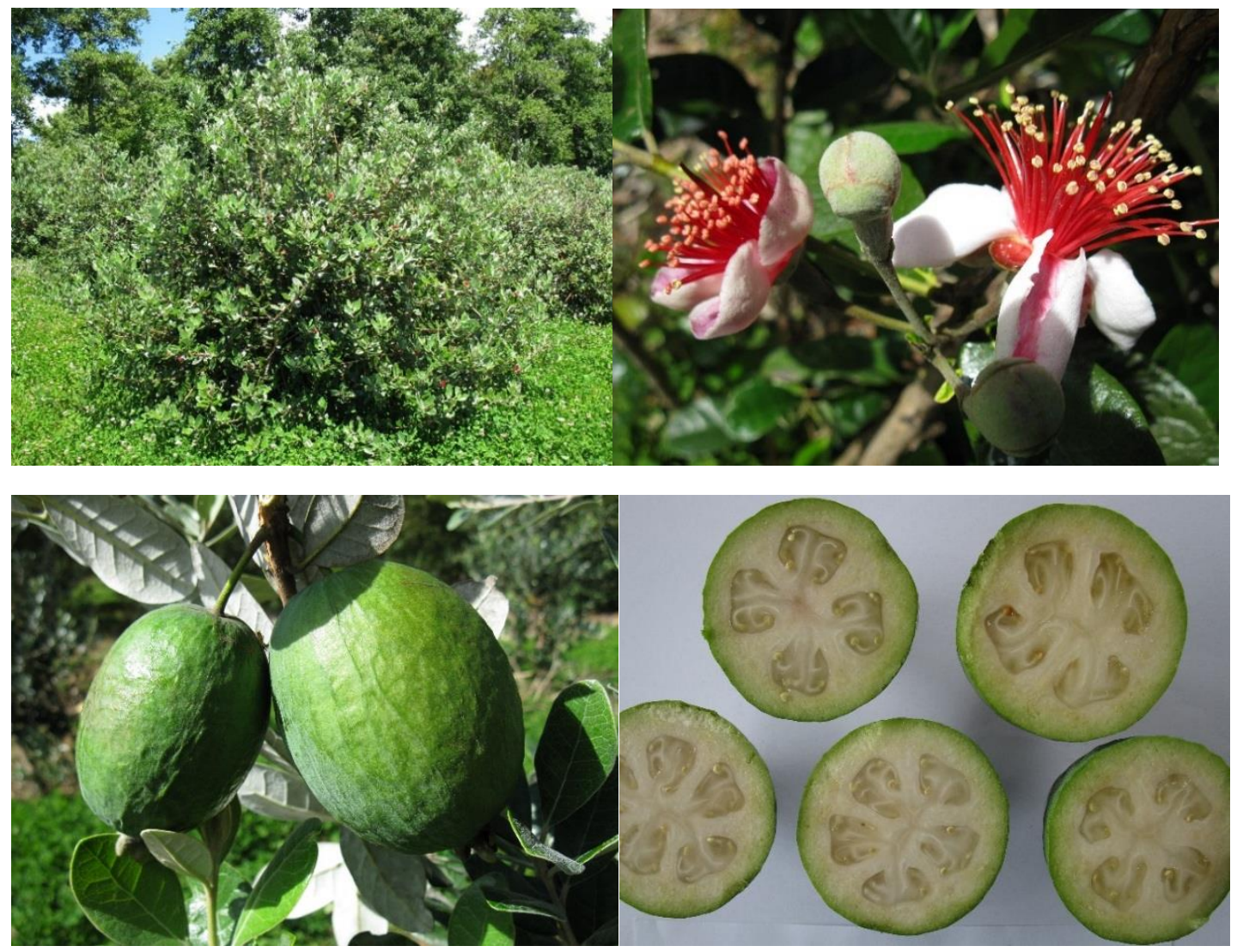

Figure 2-1. Representative images of feijoa including feijoa tree (upper left), feijoa flowers (upper right), feijoa fruit (lower left), and cross-section of feijoa fruit (lower right). Images provided by Foretaste Feijoa Fruit Ltd., Takaka, NZ.

The presence of antifungal compounds in feijoa is particularly compelling given the longstanding observation by feijoa breeders that feijoa fruit is rarely penetrated by fungal pathogens. There is thus great potential to explore feijoa peels for antifungal compounds and turn this waste product into a high value antifungal pharmaceutical. Out of the annual global 
feijoa crop (105,000 tons), approximately $40 \%$ is used in juice production that generates approximately 42,000 tons of fejoa peel waste. This is a significant amount of waste production in the feijoa industry that could potentially be used to generate additional commercial gain. While there has been extensive success in the citrus industry making use of citrus processing byproduct streams (e.g., the production of phytochemicals and nutraceuticals such as citrus molasses, D-limonene and bioflavonoids) (Shan 2016), such efforts have not yet been explored for feijoa.

Since bioactivity has been documented without reference to specific feijoa cultivars (Basile, et al. 2010; GD, et al. 2016; Hap and Gutierrez 2012; Martin, et al. 2015; Nakashima 2001; Nasef, et al. 2015; Saj, et al. 2008; Turco, et al. 2016; Weston 2010) and antifungal activity has not often been investigated, here we explored the potential of developing high value antifungal drugs from feijoa peels via compound profiling of 16 cultivars of feijoa including commercial and non-commercial cultivars. We correlated 151 volatile compounds with antifungal activity, identified two novel cultivars as those with the most antifungal activity, and determined that the compound 4-cyclopentene-1,3-dione, present in both of these cultivars was potently antifungal via the inhibition of chitin synthesis. As the antifungal mechanism of action targets chitin, which does not exist in humans or animals, this antifungal compound with pharmaceutical-grade potency and specificity has the potential to turn feijoa peel waste into a high-value product.

\subsection{MATERIALS AND METHODS}

\subsubsection{Plant material}

Fruits of 16 feijoa cultivars were grown and harvested in one location (Takaka, New Zealand): Apollo, FFF5, FFF6, FFF7, FFF8, FFF9, Kakapo, Karamea, Kawatiri, Opal Star, 
R263, Tagan, Triumph, Unique, Waingaro, and Waitui. Peels were surface-sterilized with ethanol, separated from pulp with a sterile scalpel, homogenized (Breville BLR50S Motiv Blender, Melbourne, Australia) at maximum speed for $5 \mathrm{~min}$, centrifuged at $4000 \mathrm{rpm}$ for 5 min, and the pellet was used for methanolic extraction.

\subsubsection{Compound extraction}

Compounds were extracted with methanol at a ratio of $700 \mathrm{~g}$ of homogenized peel to an equal volume of methanol. The peel:methanol mixture was extracted through shaking at 230 $\mathrm{rpm}$ at $30^{\circ} \mathrm{C}$ for $24 \mathrm{~h}$, and then centrifuged at $4000 \mathrm{rpm}$ for $5 \mathrm{~min}$. The supernatant was filtered through $0.22 \mu \mathrm{m}$ filters to ensure sterility. Methanol was removed using a cold trap system (Labconco, Kansas City, MO, USA), and the solid methanol extract was resuspended in DMSO (at a ratio of $1 \mathrm{ml}$ DMSO to $1 \mathrm{~mL}$ methanol) and stored at $-20^{\circ} \mathrm{C}$.

\subsubsection{Compound profiling}

Feijoa volatiles were analyzed on a Shimadzu QP2010-Plus gas chromatograph (Shimadzu, Kyoto, Japan) fitted with a RXI-5SilMS column (30 m x 0.25 mm i.d. x $0.25 \mu \mathrm{m}$ film thickness) (Restek, Bellefonte, PA, USA) and attached to an electron impact mass spectrometer operating at $70 \mathrm{eV}$ in positive ion mode, scanning from $\mathrm{m} / \mathrm{z}$, 42-600 every 300 msec. Samples were introduced $(1 \mu \mathrm{L})$ using an AOC-20i auto-sampler with a split injection (20:1) at an injector temperature of $270^{\circ} \mathrm{C}$ using helium as the carrier gas at a linear velocity of $43.4 \mathrm{~cm} / \mathrm{s}(1.38 \mathrm{~mL} / \mathrm{min})$ at constant flow. Each sample was injected with an initial oven temperature of $50^{\circ} \mathrm{C}$ for $2 \mathrm{~min}$, followed by a ramp at $10{ }^{\circ} \mathrm{C} / \mathrm{min}$ to $300^{\circ} \mathrm{C}$ with a final hold for 5 min. Compounds were identified by comparison of fragmentation mass spectra with those contained in the NIST11 MS library with a spectral matching similarity index (SI) $>85 \%$ in at least two of three experimental replicates. Compound annotation was performed using Linear Retention Indices (LRIs) compared with freely accessible LRI (Lemmon, et al. 2015) ( \pm 50 LRI 
units) values using similar column-types while compound identification was achieved by comparison of LRI and mass spectral fragmentation patterns with those of authentic standards based on the proposed minimum reporting standards according to the Chemical Analysis Working Group (Sumner, et al. 2007). According to this standard, we report compounds as the following: a, identified compound based on matched mass spectrum and LRI of an authentic standard; b, annotated compound with matched LRI and mass spectrum; c, annotated compound with matched mass spectrum; d, unknown.

\subsubsection{Antifungal activity and haploinsufficiency}

All strains of S. cerevisiae were obtained from Open Biosystems (Huntsville, AL, USA) in the haploid BY4741 background and diploid BY4743 background. Pathogenic Candida strains were obtained from American Type Culture Collection (Manassas, VA, USA) and included C. albicans (ATCC 10231), C. glabrata (ATCC 90030), C. parapsilosis (ATCC 90018), and C. tropicalis (ATCC 13803). Fungal growth was measured in a liquid assay in biological and technical triplicate as previously described (Ruggles, et al. 2014). Fungal cultures at $5 \times 10^{5}$ cells $/ \mathrm{mL}$ were grown in Synthetic Complete (SC) media with varying concentrations of feijoa extract or compound, incubated at $30^{\circ} \mathrm{C}$ for $14 \mathrm{~h}$, and quantified via absorbance at $600 \mathrm{~nm}$ using a Perkin Elmer Envision plate reader (Perkin Elmer, Waltham, MA, USA). Growth inhibition was calculated using the following formula:

Growth inhibition $(\%)=100-\left[\left(\mathrm{OD}_{600}\right.\right.$ of treatment/OD 600 of control $\left.) \times 100\right]$

\subsubsection{Multivariate statistical analysis}

Compound profiles and bioactivity were compared using a partial least squares regression (PLSR) analysis incorporated in UNSCRAMBLER (version X10.4, CAMO, Oslo, Norway) as previously described (Killeen, et al. 2013). The data were arranged in an X x Y 
matrix, where the response variable Y (bioactivity) corresponded to growth inhibition of $S$. cerevisiae of the 16 cultivar extracts and the predictor variable $\mathrm{X}$ corresponded to the largest peak area for each compound. All X variables were log transformed, mean centred and scaled to one standard deviation. All Y variables were mean centred. The PLSR analysis used the NonLinear Iterative Partial Least Squares (NIPALS) algorithm. Compound lists for each cultivar were generated using the weighted regression coefficient and validated using random crossvalidation. The Martens Uncertainty Test based on cross-vaidation used factors 1 and 2 to identify compounds statistically significant to the model.

\subsubsection{Antibacterial activity}

Growth inhibition of modified strains of Bacillus subtilis (ATCC 6051), a tolC mutant efflux pump-deficient Escherichia coli (W3110), an extended spectrum $\beta$-lactamase Klebsiella pneumoniae (NZRM 4387), and methicillin resistant Staphylococcus aureus (MRSA; ATCC 43300) were measured in liquid media via absorbance at $600 \mathrm{~nm}$ using an Envision plate reader (Perkin Elmer, Waltham, MA, USA) as previously described (Prosser, et al. 2010). Percentage growth inhibition was then calculated using the following formula:

Growth inhibition $(\%)=100-\left[\left(\mathrm{OD}_{600}\right.\right.$ of treatment/OD 600 of control $\left.) \times 100\right]$

\subsubsection{Fluorescent microscopy}

Chitin was visualized using the fluorescent stain Calcafluor White (Sigma) as previously described (Costa-de-Oliveira, et al. 2013). Cells were grown overnight in SC media and then grown to mid-log in SC starting at a common density $\left(\mathrm{OD}_{600}=0.1\right)$. Calcafluor White $(1 \mathrm{~g} / \mathrm{L})$ was added on the top of the cells and visualized after 5 min using Evo-Tec OPERA high throughput confocal microscope (Perkin Elmer) at 60X using the DAPI filter. 


\subsubsection{Standards}

4-Cyclopentene-1,3-dione, $\beta$-caryophyllene, (E)-3-hexenyl butyrate, and imidazole were purchased from Sigma Aldrich. Flavone was isolated from natural sources and provided as a gift (Stephen Bloor, Callaghan Innovation).

\subsection{RESULTS}

\subsubsection{Bioactivity of feijoa cultivar extracts}

The bioactivity of feijoa extracts has been examined in an undocumented set of commercial cultivars (Basile, et al. 2010; GD, et al. 2016; Hap and Gutierrez 2012; Martin, et al. 2015; Nakashima 2001; Nasef, et al. 2015; Saj, et al. 2008; Turco, et al. 2016; Weston 2010). To further understand the chemical diversity that exists within the genetic diversity of feijoa, here we investigated the bioactivity of ten commercial cultivars (Apollo, Kakapo, Karamea, Kawatiri, Opal Star, Tagan, Triumph, Unique, Waingaro, Waitui) and six novel cultivars (FFF5, FFF6, FFF7, FFF8, FFF9, R263). We quantified the efficacy of methanolic extracts derived from peels of these 16 feijoa cultivars to inhibit the growth of $S$. cerevisiae (Baker's yeast), an organism that has extensively been used to examine bioactivity of compounds and extracts (Lee, et al. 2014; Parsons, et al. 2006). Specifically, we measured the percentage of inhibition of growth of S. cerevisiae over a period of 14 hours, the amount of time that it takes an untreated wild-type strain of yeast to reach the mid-log phase of growth. Based on growth in the presence of a range of extract concentrations, we determined that $0.01 \%$ v/v of an extract significantly reduced growth of a wild-type (WT) strain of yeast (BY4741) by $18-85 \%$ compared to untreated WT (Figure. 2.2). Methanolic extracts of the commercial cultivars inhibited growth by $18-70 \%$ compared to untreated cells, while those from the novel cultivars inhibited growth by $30-85 \%$ compared to untreated cells. The most bioactive cultivars 
were the novel cultivars FFF5 and FFF6, which were each significantly different from the next most bioactive cultivar Waitui $(p<0.0005)$. These results indicate that there is extensive variation in bioactivity among feijoa cultivars as well as highlighting that the greatest bioactivity is in two novel feijoa cultivars (FFF5 and FFF6).

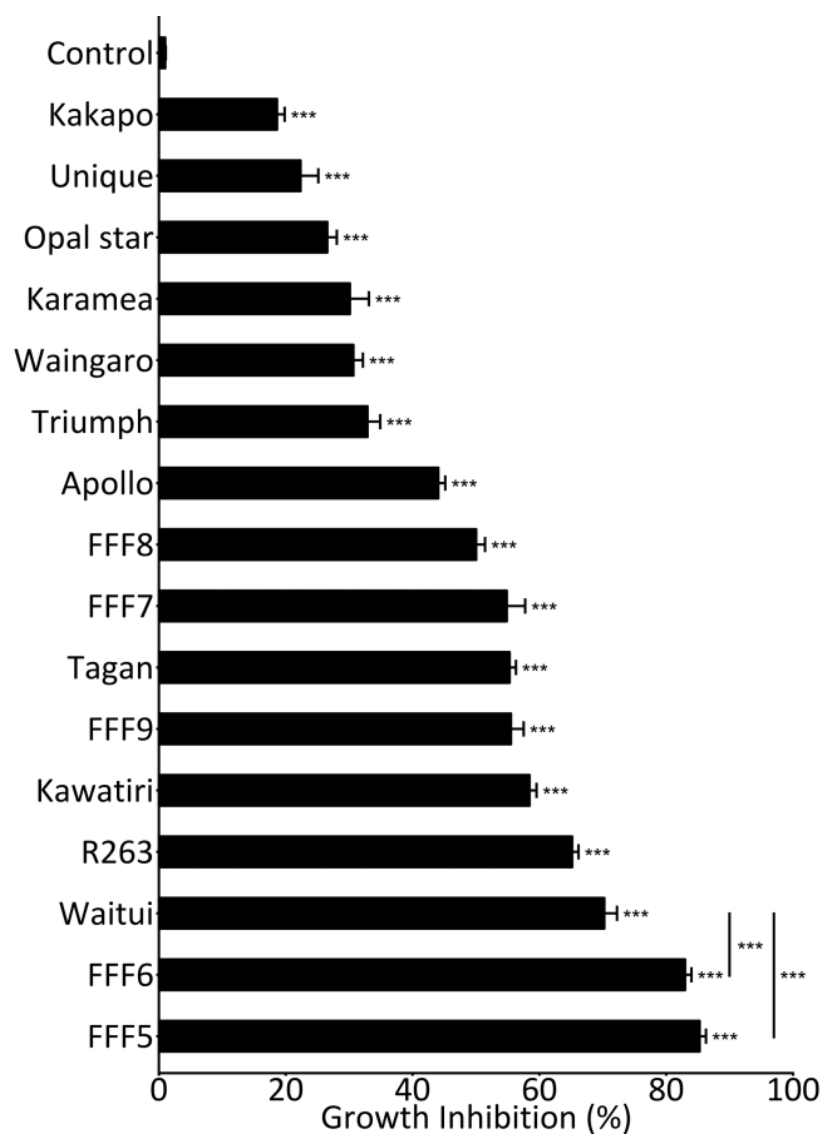

Figure 2-2. Bioactivity of methanolic extracts $(0.01 \% \mathrm{v} / \mathrm{v})$ of 16 feijoa cultivars based on growth inhibition of $S$. cerevisiae. The growth percentage inhibition was calculated via absorbance $\left(\mathrm{OD}_{600}\right)$ at mid-log in treated cells relative to untreated cells. Data shown as mean \pm S.D. ${ }^{* *}, p<0.005$, Student's $t$-test comparing treated and untreated cells. $* * *, p<0.0005$, Student's $t$-test comparing treated and untreated cells.

\subsubsection{Compound profiles of feijoa cultivars}

Antifungal bioactivity has only been reported in a few studies that explored crude acetone extracts and essential oils of feijoa (Basile, et al. 2010; GD, et al. 2016; Nakashima 2001; Saj, et al. 2008). To further define the compound diversity of feijoa in terms of antifungal 
properties, we used GC-MS to obtain unbiased metabolomic profiles in the methanolic extracts of peels from 16 feijoa cultivars. The identity of the volatile and semi-volatile compounds was tentatively assigned by comparison of mass spectral fragmentation patterns with those of more than 212,000 compounds in the NIST11 database, using a cutoff of $85 \%$ spectral similarity. Across the 16 feijoa cultivars, GC-MS analysis distinguished 151 compounds across all cultivars, of which 68 compounds were tentatively annotated on the basis of $>85 \%$ similarity to a spectrum in the NIST11 database (Figure. 2.3). Additionally, 79 compounds did not match to compounds in the NIST database, and these compounds were thus classified as unknown (Table 2.1).

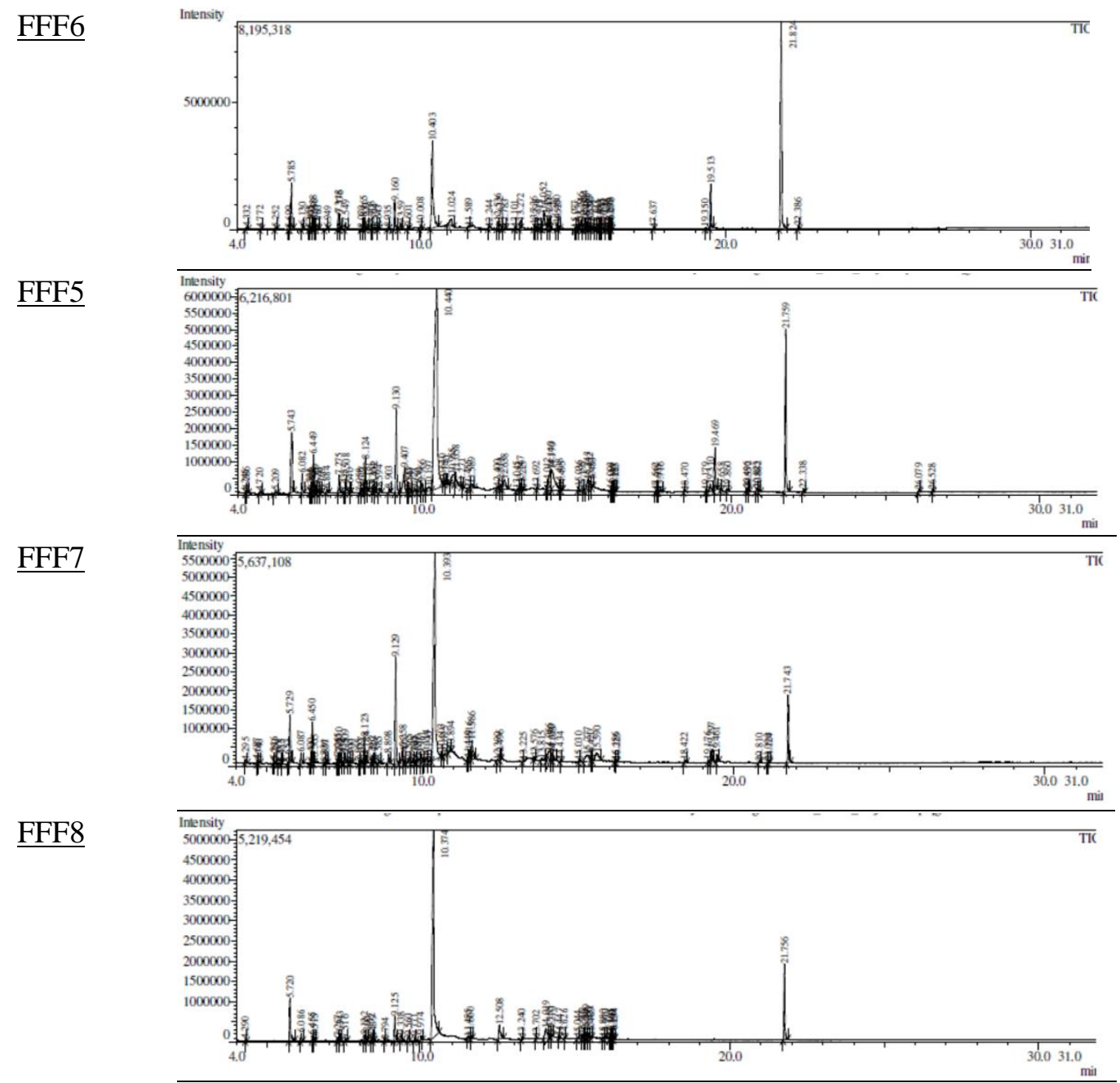


kakapo

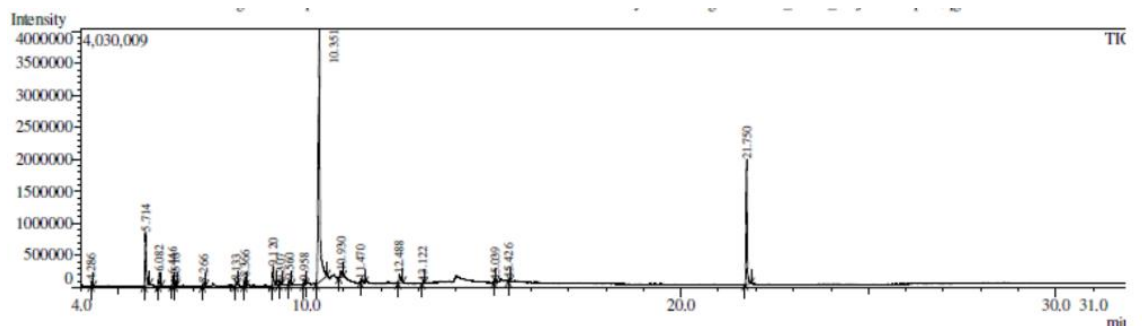

Tagan

$\underline{\text { triumph }}$

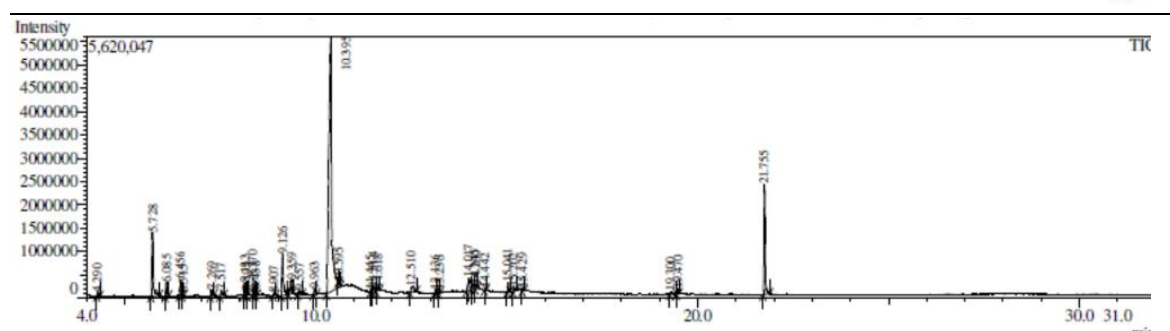

(a)

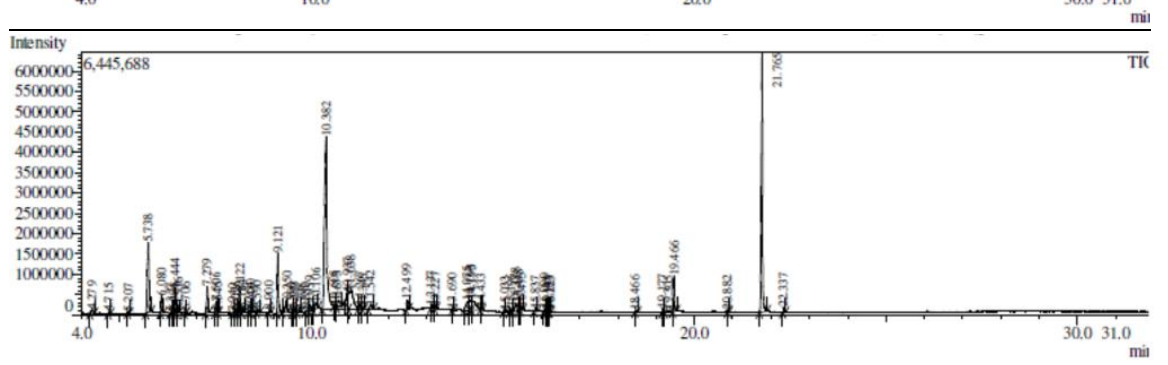

$\underline{\text { Opal star }}$

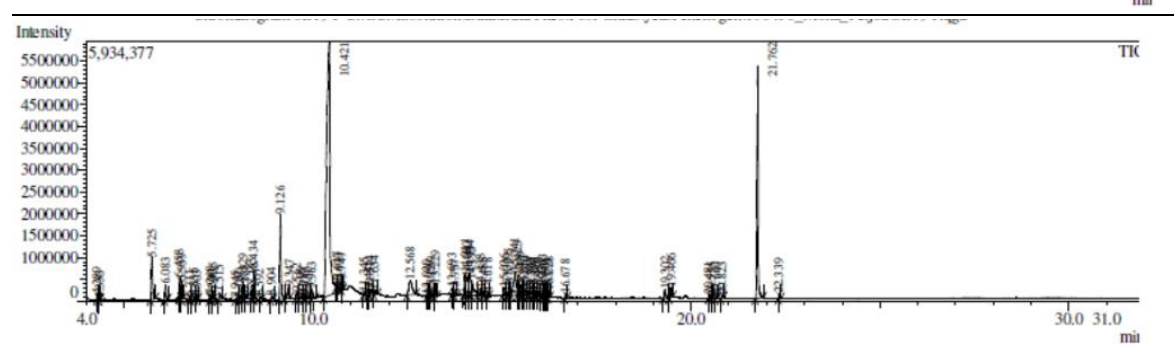

unique

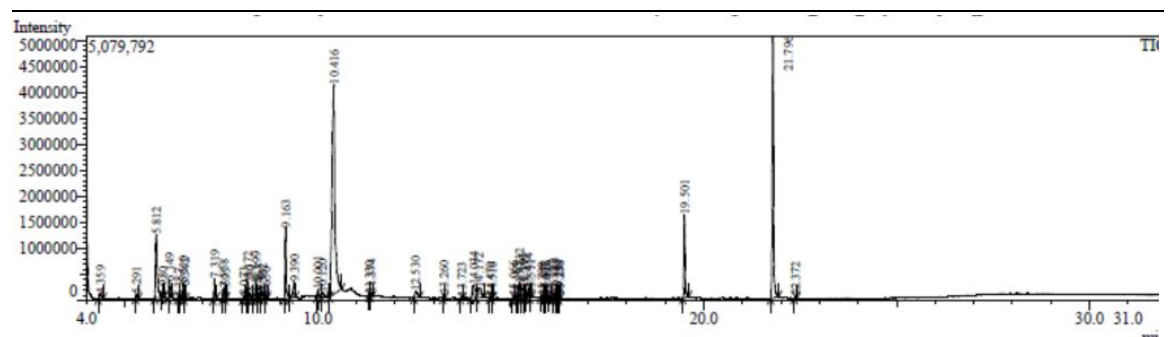

waitui

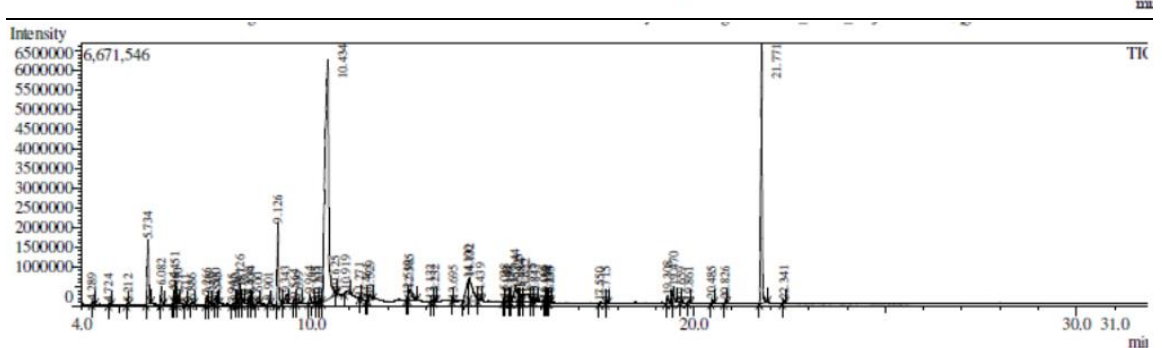

$\underline{\text { Waingaro }}$

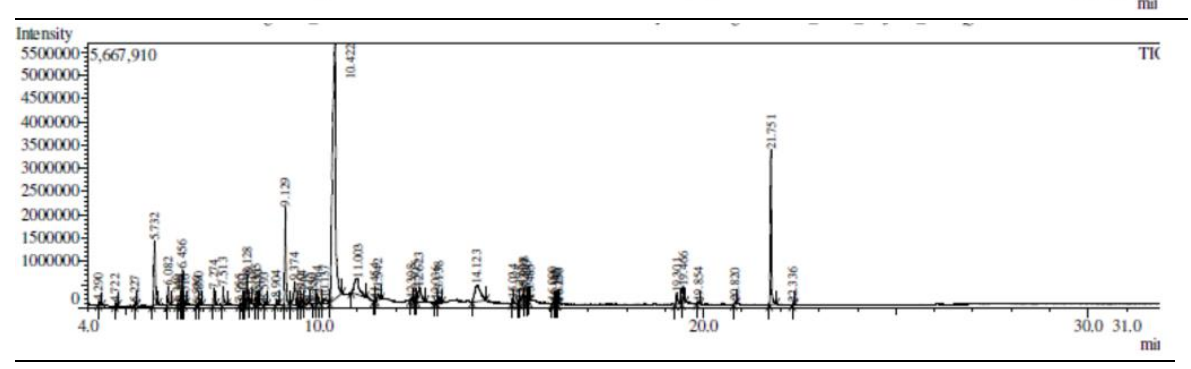




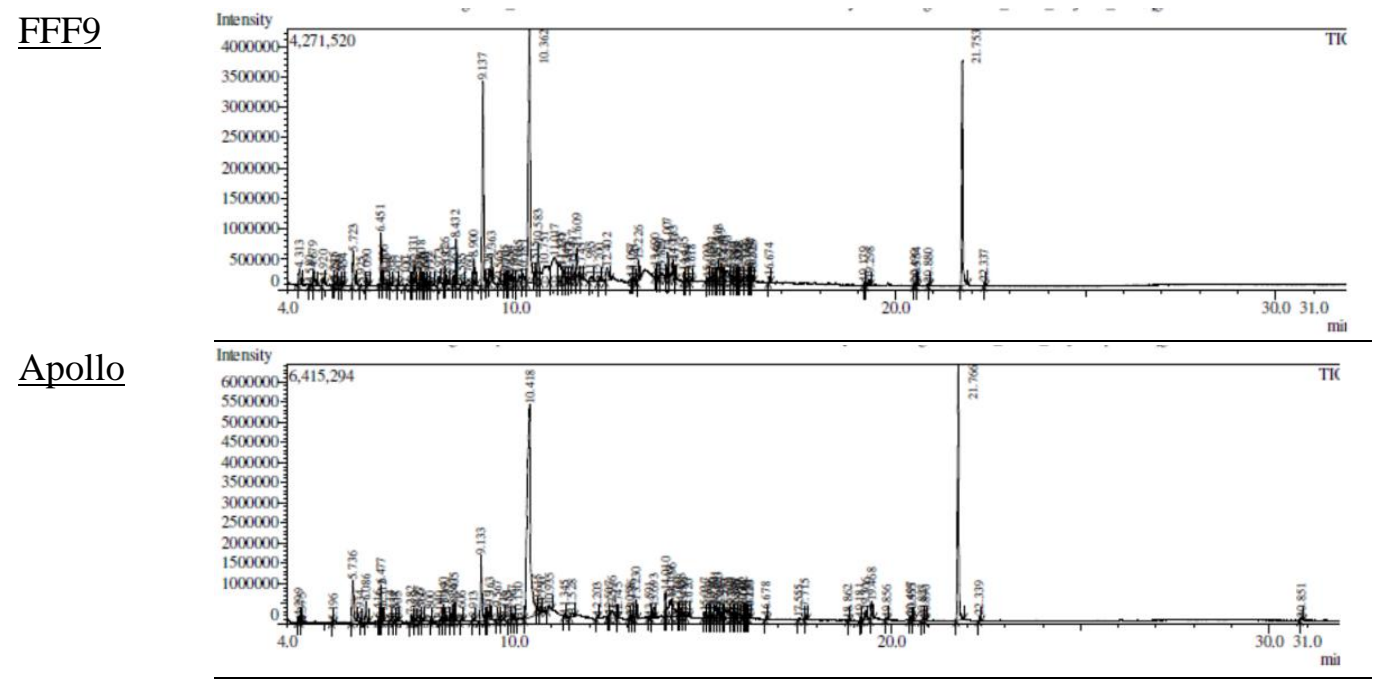

Figure 2-3. Feijoa cultivars Chromatogram

Table 2-1. List of compounds in 16 feijoa cultivars. 151 compounds detected by GC-MS and LRI and similarity to NIST database added to the table.- is no LRI found in C3; a is LRI , MS spectra and standard match; b is LRI and MS spectra match; $c$ is only MS spectra match; $d$ is none mathes with similarity search less than $85 \%$.

\begin{tabular}{llllll}
\hline $\begin{array}{l}\text { Compound } \\
\text { Number }\end{array}$ & $\begin{array}{l}\text { experi } \\
\text { mental } \\
\text { LRI }\end{array}$ & $\begin{array}{l}\text { literatu } \\
\text { re LRI }\end{array}$ & annotation & $\begin{array}{l}\text { Weighted regression } \\
\text { coefficients }\end{array}$ & $\begin{array}{l}\text { annotai } \\
\text { on level }\end{array}$ \\
\hline 1 & 597 & 851 & 3-Hexen-1-ol & 0.45 & $\mathrm{c}$ \\
\hline 2 & 630 & - & L-Lactic acid/propa... & -0.10 & $\mathrm{c}$ \\
\hline 7 & 697 & 913 & 2(5H)-Furanone & -0.24 & $\mathrm{c}$ \\
\hline 8 & 710 & 933 & 4-hydroxy-butanoic acid, & -0.01 & $\mathrm{c}$ \\
\hline 10 & 716 & - & 1,2-Cyclopentanedione & 0.00 & $\mathrm{c}$ \\
\hline 11 & 750 & 949 & 3-Methyl-2,5-furandione & -0.48 & $\mathrm{c}$ \\
\hline 13 & 784 & 949 & 5-Methyl-2-furancarboxaldehyde & -0.40 & $\mathrm{c}$ \\
\hline 17 & 819 & 977 & 2,4-Dihydroxy-2,5-dimethyl- & 0.06 & $\mathrm{c}$ \\
\hline 19 & & & 3(2H)-furan-3-one & & $\mathrm{c}$ \\
\hline 20 & 824 & 966 & 3-Octanone & 0.58 & $\mathrm{c}$ \\
\hline 4 & 836 & - & 2H-Pyran-2,6(3H)-dione & 0.47 & $\mathrm{a}$ \\
\hline 21 & 843 & 843 & 4-Cyclopentene-1,3-dione & 1.24 & $\mathrm{c}$ \\
\hline 22 & 844 & 992 & 3-Octanol & 0.11 & $\mathrm{c}$ \\
\hline 24 & 849 & - & 4-Hexen-1-ol acetate & 0.11 & $\mathrm{c}$ \\
\hline 25 & 879 & 1004 & 4-Carene & 0.36 & $\mathrm{c}$ \\
\hline 26 & 900 & - & Dihydro-3-methylene-2,5- & 0.31 & $\mathrm{c}$ \\
\hline 27 & 936 & & furandione, & & $\mathrm{c}$ \\
\hline 35 & 912 & 920 & 2,5-Hexanedione & 1.33 & -0.33 \\
\hline
\end{tabular}




\begin{tabular}{|c|c|c|c|c|c|}
\hline 39 & 948 & 1064 & g-Terpinene & 0.36 & $\mathrm{c}$ \\
\hline 36 & 963 & - & 1,3,5-Triazine-2,4,6-triamine & 0.05 & $\mathrm{c}$ \\
\hline 37 & 973 & 1076 & 2,5-Furandicarboxaldehyde & 0.17 & $\mathrm{c}$ \\
\hline 41 & 1007 & 1106 & Methyl benzoate & 0.23 & $\mathrm{c}$ \\
\hline 42 & 1015 & 1098 & 3,7-Dimethyl-1,6-octadien-3-ol & -0.11 & $\mathrm{~b}$ \\
\hline 43 & 1017 & 380 & Acetaldehyde & 0.10 & $\mathrm{c}$ \\
\hline 44 & 1031 & - & Levoglucosenone & 0.14 & $\mathrm{c}$ \\
\hline 46 & 1060 & - & $\begin{array}{l}\text { N-methyl-N-nitroso-2- } \\
\text { propanamine }\end{array}$ & 0.76 & $\mathrm{c}$ \\
\hline 47 & 1077 & 1154 & $\begin{array}{l}\text { 2,3-Dihydro-3,5-dihydroxy-6- } \\
\text { methyl-4H-pyran-4-one }\end{array}$ & 0.33 & $\mathrm{c}$ \\
\hline 49 & 1095 & 1185 & $\begin{array}{l}2(3 \mathrm{H}) \text {-Furanone, dihydro-4- } \\
\text { hydroxy- }\end{array}$ & -0.10 & $\mathrm{c}$ \\
\hline 50 & 1106 & 1191 & Benzoic acid & -0.02 & $\mathrm{c}$ \\
\hline 55 & 1141 & 1220 & $\begin{array}{l}\text { 3,5-Dihydroxy-2-methyl-4H- } \\
\text { pyran-4-one }\end{array}$ & 0.21 & c \\
\hline 57 & 1146 & 1219 & Catechol & -0.34 & $\mathrm{c}$ \\
\hline 58 & 1146 & 1185 & trans-3-Hexenyl butanoate & 0.84 & $\mathrm{~b}$ \\
\hline 59 & 1156 & 1170 & $\begin{array}{l}\text { 4-Methyl-1-(1-methylethyl)-3- } \\
\text { cyclohexen-1-ol }\end{array}$ & 0.81 & $\mathrm{~b}$ \\
\hline 60 & 1162 & 1159 & 2-Pentanyl propanoate & 0.46 & $\mathrm{~b}$ \\
\hline 63 & 1184 & - & $\begin{array}{l}\text { Tetrahydrofuran-5-on-2- } \\
\text { methanol, a-[a-methoxy- } \\
\text { (tetrahydrofuran-5-on-2- } \\
\text { ylmethoxy)]-hydroxyfuran }\end{array}$ & -0.10 & $\mathrm{c}$ \\
\hline 64 & 1185 & - & $\begin{array}{l}\text { 1,4:3,6-Dianhydro-a- } \\
\text { glucopyranose }\end{array}$ & 0.09 & $\mathrm{c}$ \\
\hline 65 & 1205 & 1224 & 5-Hydroxymethylfurfural & 0.33 & $\mathrm{~b}$ \\
\hline 67 & 1226 & - & 2,3-Propanediol-1-acetate & 0.30 & $\mathrm{c}$ \\
\hline 69 & 1239 & 1255 & Geraniol & 0.41 & $\mathrm{~b}$ \\
\hline 68 & 1240 & - & $\begin{array}{l}\text { trans-2-methyl-But-2-enedioic } \\
\text { acid }\end{array}$ & -0.06 & $\mathrm{c}$ \\
\hline 74 & 1268 & - & Methylene-butanedioic acid & 0.05 & $\mathrm{c}$ \\
\hline 80 & 1282 & 1160 & $\begin{array}{l}\text { 2,3-Dihydro-3,5-dihydroxy-6- } \\
\text { methyl-4H-pyran-4-one }\end{array}$ & -0.10 & $\mathrm{c}$ \\
\hline 81 & 1285 & 1263 & 3-Methyl-1,2-benzenediol & -0.07 & $\mathrm{~b}$ \\
\hline 76 & 1294 & 1490 & 2-Tridecanone & -0.32 & $\mathrm{c}$ \\
\hline 78 & 1297 & 1311 & Salicylic acid & -0.72 & $\mathrm{~b}$ \\
\hline 84 & 1308 & 1133 & Maltol & 0.42 & $\mathrm{c}$ \\
\hline 93 & 1433 & 1412 & b-Bourbonene & 0.39 & $\mathrm{~b}$ \\
\hline 94 & 1466 & 1451 & 4-Methoxybenzoic acid & -0.13 & $\mathrm{~b}$ \\
\hline 95 & 1482 & 1451 & b-Caryophyllene & 0.92 & a \\
\hline 98 & 1522 & 1497 & Humulene & 0.54 & $\mathrm{~b}$ \\
\hline 100 & 1543 & 1558 & 4-Hydroxybenzoic acid & -0.01 & $\mathrm{~b}$ \\
\hline 105 & 1561 & 1530 & $\begin{array}{l}\text { 1H-Cycloprop[e]azulene, } \\
\text { 1a,2,3,5,6,7,7a,7b-octahydro- } \\
\text { 1,1,4,7-tetramethyl-, [1aR- } \\
\text { (1a.alpha.,7.alpha.,7a.beta.,7b.al } \\
\text { pha.)]- }\end{array}$ & 1.04 & $\mathrm{~b}$ \\
\hline 103 & 1562 & 1481 & $\begin{array}{l}\text { 1-Methyl-5-methylene-8-(1- } \\
\text { methylethyl)-1,6-cyclodecadiene }\end{array}$ & 0.03 & c \\
\hline 102 & 1575 & - & Allose & 0.51 & $\mathrm{c}$ \\
\hline 106 & 1601 & 1566 & $\begin{array}{l}\text { Naphthalene, 1,2,3,4-tetrahydro- } \\
\text { 1,6-dimethyl-4-(1-methylethyl)-, }\end{array}$ & 0.42 & $\mathrm{~b}$ \\
\hline 107 & 1660 & 1588 & 3-Hexen-1-yl benzoate & 0.42 & c \\
\hline
\end{tabular}




\begin{tabular}{|c|c|c|c|c|c|}
\hline 111 & 1691 & - & 1,6-Anhydro-b-glucofuranose & 0.41 & $\mathrm{c}$ \\
\hline 113 & 1700 & 1550 & $\begin{array}{l}\text { 1H-Cycloprop[e]azulen-4-ol, } \\
\text { decahydro-1,1,4,7-tetramethyl-, } \\
\text { [1aR- } \\
\text { (1a.alpha.,4.beta.,4a.beta.,7.alpha } \\
\text {.,7a.beta.,7b.alpha.)]- }\end{array}$ & 0.96 & $\mathrm{c}$ \\
\hline 118 & 1720 & - & Methyl b-glucopyranoside & 0.62 & $\mathrm{c}$ \\
\hline 121 & 1739 & 1650 & $\begin{array}{l}\text { 1H-Cycloprop[e]azulen-7-ol, } \\
\text { decahydro-1,1,7-trimethyl-4- } \\
\text { methylene-, } \\
\text { (1a.alpha.,4a.alpha.,7.beta.,7a.bet } \\
\text { a.,7b.alpha.)]- }\end{array}$ & 0.52 & $\mathrm{c}$ \\
\hline 124 & 1749 & 1655 & a-Cadinol & 0.07 & $\mathrm{c}$ \\
\hline 128 & 1825 & 1740 & $\begin{array}{l}\text { 3,7,11-Trimethyl-2,6,10- } \\
\text { dodecatrien-1-ol }\end{array}$ & -0.08 & $\mathrm{c}$ \\
\hline 132 & 2036 & 1996 & Methyl 15-methylhexadecanoate & -0.31 & $\mathrm{~b}$ \\
\hline 138 & 2194 & 2170 & $\begin{array}{l}\text { Methyl }(\mathrm{Z}, \mathrm{Z}, \mathrm{Z})-9,12,15- \\
\text { octadecatrienoate }\end{array}$ & -0.29 & $\mathrm{c}$ \\
\hline 140 & 2242 & 2154 & $\begin{array}{l}(\mathrm{Z}, \mathrm{Z}, \mathrm{Z})-9,12,15- \\
\text { Octadecatrienoic acid }\end{array}$ & -0.79 & $\mathrm{c}$ \\
\hline 143 & 2264 & - & [(Hexadecyloxy)methyl]-oxirane & 0.09 & $\mathrm{c}$ \\
\hline 141 & 2323 & - & Flavone & 1.31 & $\mathrm{a}$ \\
\hline 3 & $\begin{array}{l}\text { unknow } \\
\text { n }\end{array}$ & $\begin{array}{l}\text { unknow } \\
\mathrm{n}\end{array}$ & unknown & -0.01 & $\mathrm{~d}$ \\
\hline 5 & $\begin{array}{l}\text { unknow } \\
\mathrm{n}\end{array}$ & $\begin{array}{l}\text { unknow } \\
\text { n }\end{array}$ & unknown & -0.10 & d \\
\hline 6 & $\begin{array}{l}\text { unknow } \\
\text { n }\end{array}$ & $\begin{array}{l}\text { unknow } \\
\text { n }\end{array}$ & unknown & 0.01 & $\mathrm{~d}$ \\
\hline 9 & $\begin{array}{l}\text { unknow } \\
\text { n }\end{array}$ & $\begin{array}{l}\text { unknow } \\
\text { n }\end{array}$ & unknown & 0.00 & $\mathrm{~d}$ \\
\hline 12 & $\begin{array}{l}\text { unknow } \\
\mathrm{n}\end{array}$ & $\begin{array}{l}\text { unknow } \\
\text { n }\end{array}$ & unknown & -0.63 & $\mathrm{~d}$ \\
\hline 14 & $\begin{array}{l}\text { unknow } \\
\mathrm{n}\end{array}$ & $\begin{array}{l}\text { unknow } \\
\text { n }\end{array}$ & unknown & -0.48 & $\mathrm{~d}$ \\
\hline 15 & $\begin{array}{l}\text { unknow } \\
\mathrm{n}\end{array}$ & $\begin{array}{l}\text { unknow } \\
\text { n }\end{array}$ & unknown & 0.36 & d \\
\hline 16 & $\begin{array}{l}\text { unknow } \\
\mathrm{n}\end{array}$ & $\begin{array}{l}\text { unknow } \\
\text { n }\end{array}$ & unknown & -0.04 & d \\
\hline 18 & $\begin{array}{l}\text { unknow } \\
\text { n }\end{array}$ & $\begin{array}{l}\text { unknow } \\
\text { n }\end{array}$ & unknown & -0.60 & $\mathrm{~d}$ \\
\hline 23 & $\begin{array}{l}\text { unknow } \\
\text { n }\end{array}$ & $\begin{array}{l}\text { unknow } \\
\text { n }\end{array}$ & unknown & 0.50 & $\mathrm{~d}$ \\
\hline 29 & $\begin{array}{l}\text { unknow } \\
\text { n }\end{array}$ & $\begin{array}{l}\text { unknow } \\
\mathrm{n}\end{array}$ & unknown & -0.72 & $\mathrm{~d}$ \\
\hline 30 & $\begin{array}{l}\text { unknow } \\
\text { n }\end{array}$ & $\begin{array}{l}\text { unknow } \\
\text { n }\end{array}$ & unknown & 0.67 & $\mathrm{~d}$ \\
\hline 31 & $\begin{array}{l}\text { unknow } \\
\text { n }\end{array}$ & $\begin{array}{l}\text { unknow } \\
\text { n }\end{array}$ & unknown & -0.10 & $\mathrm{~d}$ \\
\hline 32 & $\begin{array}{l}\text { unknow } \\
\text { n }\end{array}$ & $\begin{array}{l}\text { unknow } \\
\text { n }\end{array}$ & unknown & 0.03 & d \\
\hline 34 & $\begin{array}{l}\text { unknow } \\
\text { n }\end{array}$ & $\begin{array}{l}\text { unknow } \\
\text { n }\end{array}$ & unknown & -0.32 & $\mathrm{~d}$ \\
\hline 40 & $\begin{array}{l}\text { unknow } \\
\mathrm{n}\end{array}$ & $\begin{array}{l}\text { unknow } \\
\mathrm{n}\end{array}$ & unknown & 0.66 & $\mathrm{~d}$ \\
\hline 45 & $\begin{array}{l}\text { unknow } \\
\mathrm{n}\end{array}$ & $\begin{array}{l}\text { unknow } \\
\mathrm{n}\end{array}$ & unknown & -0.10 & $\mathrm{~d}$ \\
\hline 48 & $\begin{array}{l}\text { unknow } \\
\mathrm{n}\end{array}$ & $\begin{array}{l}\text { unknow } \\
\mathrm{n}\end{array}$ & unknown & -0.31 & d \\
\hline
\end{tabular}




\begin{tabular}{|c|c|c|c|c|}
\hline 52 & $\begin{array}{l}\text { unknow } \\
\mathrm{n}\end{array}$ & $\begin{array}{l}\text { unknow unknown } \\
\text { n }\end{array}$ & -0.80 & d \\
\hline 54 & $\begin{array}{l}\text { unknow } \\
\mathrm{n}\end{array}$ & $\begin{array}{l}\text { unknow unknown } \\
\mathrm{n}\end{array}$ & -0.52 & d \\
\hline 56 & $\begin{array}{l}\text { unknow } \\
\mathrm{n}\end{array}$ & $\begin{array}{l}\text { unknow unknown } \\
\text { n }\end{array}$ & -0.10 & d \\
\hline 61 & $\begin{array}{l}\text { unknow } \\
\mathrm{n}\end{array}$ & $\begin{array}{l}\text { unknow unknown } \\
\mathrm{n}\end{array}$ & -0.91 & $d$ \\
\hline 62 & $\begin{array}{l}\text { unknow } \\
\mathrm{n}\end{array}$ & $\begin{array}{l}\text { unknow unknown } \\
\mathrm{n}\end{array}$ & 0.36 & d \\
\hline 66 & $\begin{array}{l}\text { unknow } \\
\mathrm{n}\end{array}$ & $\begin{array}{l}\text { unknow unknown } \\
\text { n }\end{array}$ & -0.10 & d \\
\hline 72 & $\begin{array}{l}\text { unknow } \\
\mathrm{n}\end{array}$ & $\begin{array}{l}\text { unknow unknown } \\
\mathrm{n}\end{array}$ & -0.38 & $d$ \\
\hline 73 & $\begin{array}{l}\text { unknow } \\
\mathrm{n}\end{array}$ & $\begin{array}{l}\text { unknow unknown } \\
\text { n }\end{array}$ & -0.60 & d \\
\hline 75 & $\begin{array}{l}\text { unknow } \\
\mathrm{n}\end{array}$ & $\begin{array}{l}\text { unknow unknown } \\
\mathrm{n}\end{array}$ & -0.80 & d \\
\hline 77 & $\begin{array}{l}\text { unknow } \\
\mathrm{n}\end{array}$ & $\begin{array}{l}\text { unknow unknown } \\
\mathrm{n}\end{array}$ & 0.01 & d \\
\hline 79 & $\begin{array}{l}\text { unknow } \\
\mathrm{n}\end{array}$ & $\begin{array}{l}\text { unknow unknown } \\
\text { n }\end{array}$ & 0.01 & d \\
\hline 82 & $\begin{array}{l}\text { unknow } \\
\mathrm{n}\end{array}$ & $\begin{array}{l}\text { unknow unknown } \\
\mathrm{n}\end{array}$ & -0.21 & d \\
\hline 83 & $\begin{array}{l}\text { unknow } \\
\mathrm{n}\end{array}$ & $\begin{array}{l}\text { unknow unknown } \\
\text { n }\end{array}$ & 0.41 & d \\
\hline 85 & $\begin{array}{l}\text { unknow } \\
\mathrm{n}\end{array}$ & $\begin{array}{l}\text { unknow unknown } \\
\text { n }\end{array}$ & 0.08 & d \\
\hline 86 & $\begin{array}{l}\text { unknow } \\
\mathrm{n}\end{array}$ & $\begin{array}{l}\text { unknow unknown } \\
\mathrm{n}\end{array}$ & 0.72 & d \\
\hline 87 & $\begin{array}{l}\text { unknow } \\
\mathrm{n}\end{array}$ & $\begin{array}{l}\text { unknow unknown } \\
\mathrm{n}\end{array}$ & -0.10 & d \\
\hline 88 & $\begin{array}{l}\text { unknow } \\
\mathrm{n}\end{array}$ & $\begin{array}{l}\text { unknow unknown } \\
\mathrm{n}\end{array}$ & -0.10 & d \\
\hline 89 & $\begin{array}{l}\text { unknow } \\
\mathrm{n}\end{array}$ & $\begin{array}{l}\text { unknow unknown } \\
\mathrm{n}\end{array}$ & 0.08 & d \\
\hline 90 & $\begin{array}{l}\text { unknow } \\
\text { n }\end{array}$ & $\begin{array}{l}\text { unknow unknown } \\
\mathrm{n}\end{array}$ & -0.54 & d \\
\hline 91 & $\begin{array}{l}\text { unknow } \\
\mathrm{n}\end{array}$ & $\begin{array}{l}\text { unknow unknown } \\
\mathrm{n}\end{array}$ & 0.26 & d \\
\hline 92 & $\begin{array}{l}\text { unknow } \\
\mathrm{n}\end{array}$ & $\begin{array}{l}\text { unknow unknown } \\
\mathrm{n}\end{array}$ & 0.27 & d \\
\hline 96 & $\begin{array}{l}\text { unknow } \\
\mathrm{n}\end{array}$ & $\begin{array}{l}\text { unknow unknown } \\
\mathrm{n}\end{array}$ & 0.12 & d \\
\hline 99 & $\begin{array}{l}\text { unknow } \\
\mathrm{n}\end{array}$ & $\begin{array}{l}\text { unknow unknown } \\
\mathrm{n}\end{array}$ & -0.31 & d \\
\hline 101 & $\begin{array}{l}\text { unknow } \\
\mathrm{n}\end{array}$ & $\begin{array}{l}\text { unknow unknown } \\
\mathrm{n}\end{array}$ & 0.88 & $d$ \\
\hline 104 & $\begin{array}{l}\text { unknow } \\
\mathrm{n}\end{array}$ & $\begin{array}{l}\text { unknow unknown } \\
\mathrm{n}\end{array}$ & -0.31 & d \\
\hline 108 & $\begin{array}{l}\text { unknow } \\
\mathrm{n}\end{array}$ & $\begin{array}{l}\text { unknow unknown } \\
\text { n }\end{array}$ & 0.22 & d \\
\hline 109 & $\begin{array}{l}\text { unknow } \\
\mathrm{n}\end{array}$ & $\begin{array}{l}\text { unknow unknown } \\
\mathrm{n}\end{array}$ & -0.27 & d \\
\hline 110 & $\begin{array}{l}\text { unknow } \\
\text { n }\end{array}$ & $\begin{array}{l}\text { unknow unknown } \\
\mathrm{n}\end{array}$ & -0.04 & d \\
\hline 112 & $\begin{array}{l}\text { unknow } \\
\mathrm{n}\end{array}$ & $\begin{array}{l}\text { unknow unknown } \\
\mathrm{n}\end{array}$ & 0.19 & d \\
\hline 114 & $\begin{array}{l}\text { unknow } \\
\mathrm{n}\end{array}$ & $\begin{array}{l}\text { unknow unknown } \\
\mathrm{n}\end{array}$ & 0.20 & d \\
\hline
\end{tabular}




\begin{tabular}{|c|c|c|c|c|}
\hline 115 & $\begin{array}{l}\text { unknow } \\
\text { n }\end{array}$ & $\begin{array}{l}\text { unknow unknown } \\
\mathrm{n}\end{array}$ & 0.23 & $d$ \\
\hline 116 & $\begin{array}{l}\text { unknow } \\
\mathrm{n}\end{array}$ & $\begin{array}{l}\text { unknow unknown } \\
\text { n }\end{array}$ & 0.84 & d \\
\hline 117 & $\begin{array}{l}\text { unknow } \\
\mathrm{n}\end{array}$ & $\begin{array}{l}\text { unknow unknown } \\
\mathrm{n}\end{array}$ & 0.77 & d \\
\hline 119 & $\begin{array}{l}\text { unknow } \\
\mathrm{n}\end{array}$ & $\begin{array}{l}\text { unknow unknown } \\
\mathrm{n}\end{array}$ & 0.83 & $d$ \\
\hline 120 & $\begin{array}{l}\text { unknow } \\
\mathrm{n}\end{array}$ & $\begin{array}{l}\text { unknow unknown } \\
\text { n }\end{array}$ & 0.01 & d \\
\hline 122 & $\begin{array}{l}\text { unknow } \\
\mathrm{n}\end{array}$ & $\begin{array}{l}\text { unknow unknown } \\
\mathrm{n}\end{array}$ & 1.03 & d \\
\hline 123 & $\begin{array}{l}\text { unknow } \\
\mathrm{n}\end{array}$ & $\begin{array}{l}\text { unknow unknown } \\
\mathrm{n}\end{array}$ & 0.35 & d \\
\hline 125 & $\begin{array}{l}\text { unknow } \\
\mathrm{n}\end{array}$ & $\begin{array}{l}\text { unknow unknown } \\
\mathrm{n}\end{array}$ & -0.45 & d \\
\hline 126 & $\begin{array}{l}\text { unknow } \\
\mathrm{n}\end{array}$ & $\begin{array}{l}\text { unknow unknown } \\
\text { n }\end{array}$ & 0.22 & d \\
\hline 127 & $\begin{array}{l}\text { unknow } \\
\mathrm{n}\end{array}$ & $\begin{array}{l}\text { unknow unknown } \\
\mathrm{n}\end{array}$ & -0.27 & d \\
\hline 129 & $\begin{array}{l}\text { unknow } \\
\mathrm{n}\end{array}$ & $\begin{array}{l}\text { unknow unknown } \\
\mathrm{n}\end{array}$ & 0.38 & d \\
\hline 130 & $\begin{array}{l}\text { unknow } \\
\mathrm{n}\end{array}$ & $\begin{array}{l}\text { unknow unknown } \\
\text { n }\end{array}$ & -0.16 & d \\
\hline 131 & $\begin{array}{l}\text { unknow } \\
\text { n }\end{array}$ & $\begin{array}{l}\text { unknow unknown } \\
\mathrm{n}\end{array}$ & -0.72 & d \\
\hline 133 & $\begin{array}{l}\text { unknow } \\
\mathrm{n}\end{array}$ & $\begin{array}{l}\text { unknow unknown } \\
\mathrm{n}\end{array}$ & -0.20 & d \\
\hline 134 & $\begin{array}{l}\text { unknow } \\
\mathrm{n}\end{array}$ & $\begin{array}{l}\text { unknow unknown } \\
\mathrm{n}\end{array}$ & -0.61 & d \\
\hline 135 & $\begin{array}{l}\text { unknow } \\
\mathrm{n}\end{array}$ & $\begin{array}{l}\text { unknow unknown } \\
\text { n }\end{array}$ & 0.43 & d \\
\hline 136 & $\begin{array}{l}\text { unknow } \\
\mathrm{n}\end{array}$ & $\begin{array}{l}\text { unknow unknown } \\
\mathrm{n}\end{array}$ & -0.38 & d \\
\hline 137 & $\begin{array}{l}\text { unknow } \\
\mathrm{n}\end{array}$ & $\begin{array}{l}\text { unknow unknown } \\
\mathrm{n}\end{array}$ & 0.28 & d \\
\hline 139 & $\begin{array}{l}\text { unknow } \\
\mathrm{n}\end{array}$ & $\begin{array}{l}\text { unknow unknown } \\
\mathrm{n}\end{array}$ & 0.11 & d \\
\hline 142 & $\begin{array}{l}\text { unknow } \\
\mathrm{n}\end{array}$ & $\begin{array}{l}\text { unknow unknown } \\
\mathrm{n}\end{array}$ & 0.09 & d \\
\hline 144 & $\begin{array}{l}\text { unknow } \\
\mathrm{n}\end{array}$ & $\begin{array}{l}\text { unknow unknown } \\
\text { n }\end{array}$ & 0.37 & d \\
\hline 145 & $\begin{array}{l}\text { unknow } \\
\mathrm{n}\end{array}$ & $\begin{array}{l}\text { unknow unknown } \\
\text { n }\end{array}$ & -0.60 & d \\
\hline 146 & $\begin{array}{l}\text { unknow } \\
\text { n }\end{array}$ & $\begin{array}{l}\text { unknow unknown } \\
\text { n }\end{array}$ & -0.60 & d \\
\hline 147 & $\begin{array}{l}\text { unknow } \\
\mathrm{n}\end{array}$ & $\begin{array}{l}\text { unknow unknown } \\
\mathrm{n}\end{array}$ & -0.31 & d \\
\hline 148 & $\begin{array}{l}\text { unknow } \\
\mathrm{n}\end{array}$ & $\begin{array}{l}\text { unknow unknown } \\
\mathrm{n}\end{array}$ & 0.88 & $\mathrm{~d}$ \\
\hline 149 & $\begin{array}{l}\text { unknow } \\
\text { n }\end{array}$ & $\begin{array}{l}\text { unknow unknown } \\
\mathrm{n}\end{array}$ & 1.26 & $\mathrm{~d}$ \\
\hline 151 & $\begin{array}{l}\text { unknow } \\
\mathrm{n}\end{array}$ & $\begin{array}{l}\text { unknow unknown } \\
\text { n }\end{array}$ & 0.26 & $\mathrm{~d}$ \\
\hline 71 & $\begin{array}{l}\text { unknow } \\
\mathrm{n}\end{array}$ & $\begin{array}{l}\text { unknow unknown } \\
\text { n }\end{array}$ & 0.09 & $\mathrm{~d}$ \\
\hline 97 & $\begin{array}{l}\text { unknow } \\
\mathrm{n}\end{array}$ & $\begin{array}{l}\text { unknow unknown } \\
\mathrm{n}\end{array}$ & 0.28 & $\mathrm{~d}$ \\
\hline 53 & $\begin{array}{l}\text { unknow } \\
\mathrm{n}\end{array}$ & $\begin{array}{l}\text { unknow unknown } \\
\mathrm{n}\end{array}$ & 0.87 & $\mathrm{~d}$ \\
\hline
\end{tabular}




\begin{tabular}{|c|c|c|c|c|}
\hline 51 & $\begin{array}{l}\text { unknow } \\
\mathrm{n}\end{array}$ & $\begin{array}{l}\text { unknow unknown } \\
\text { n }\end{array}$ & 0.08 & d \\
\hline 70 & $\begin{array}{l}\text { unknow } \\
\text { n }\end{array}$ & $\begin{array}{l}\text { unknow unknown } \\
\mathrm{n}\end{array}$ & 0.84 & d \\
\hline 150 & $\begin{array}{l}\text { unknow } \\
\mathrm{n}\end{array}$ & $\begin{array}{l}\text { unknow unknown } \\
\mathrm{n}\end{array}$ & 0.88 & d \\
\hline 33 & $\begin{array}{l}\text { unknow } \\
\mathrm{n}\end{array}$ & $\begin{array}{l}\text { unknow unknown } \\
\mathrm{n}\end{array}$ & -0.31 & d \\
\hline
\end{tabular}

\subsubsection{Multivariate analysis of cultivars, bioactivity, and compounds}

To investigate possible relationships between bioactivity and compound profiles of the 16 feijoa cultivars, we conducted a PLSR analysis incorporated in the software UNSCRAMBLER that is regularly used to correlate compounds and different properties (Coutinho, et al. 2016). We correlated a large number of dependent (151 compounds) and independent (bioactivity) variables for all 16 cultivars. The PLSR analysis generated a $\beta$ coefficiency value for each compound (ranging from -1 to +1 ) wherein compounds with higher positive $\beta$-coefficiency values were more correlated to bioactivity and negative $\beta$-coefficiency values were negatively correlated to bioactivity. The $\beta$-coefficiency values were then used to build seven different models (factors) to link bioactivity to the compounds. Notably, the first two factors explained $92.6 \%$ of the variance (i.e., $\mathrm{X}$ axis is the model factor 1 that explained $73 \%$ of the data variance and $\mathrm{Y}$ axis is model factor 2 that explained $20 \%$ of the data variance) in our data correlating bioactivity and compound identity, a value that is consistent with results in other studies using just the first two factors (Song, et al. 2014).

Next, we sought to correlate each cultivar with bioactivity using the first two factors. In agreement with Figure 2.2, the FFF5 cultivar was most correlated to bioactivity with FFF6 as the second closest cultivar, followed by Waitui and R263. Based on the $\beta$-coefficiency values, these four cultivars were positively correlated with bioactivity in both factor 1 and 2 . In contrast, three cultivars (FFF7, Apollo, Kawatiri, FFF9) were positively correlated to factor 1 
and negatively correlated to factor 2, while four cultivars (FFF8, Tagan, Kakapo, Karamea) were positively correlated to factor 2 and negatively correlated to factor 1 . Four cultivars (Unique, Opal Star, Triumph, Waingaro) were negatively correlated to both factors.

We then sought to distinguish the compounds that were positively and negatively correlated with bioactivity. The multivariate PLSR analysis determined those compounds to the right side of the mid-line as being positively correlated to factor 1 , while those compounds to the top side of the mid-line were positively correlated to factor 2 (Figure 2.4B). The data analysis illustrated there were 17 compounds negatively correlated to both factors 1 and 2 , while there were 26 compounds positively correlated to both factors. To define the compounds that were significantly correlated with bioactivity, we conducted further statistical analyses using a Martens uncertainty test based on the first two factors in the PLSR analysis. We detected 22 compounds that were significantly correlated with bioactivity (18 positively correlated, four negatively correlated), nine of which matched with $>85 \%$ SI to compounds in the NIST11 database (Table 2.2). Using the minimum criterion of $85 \%$ similarity to the NIST11 database for identification, we tentatively annotated nine significantly positively correlated compounds based on $\beta$-coefficiency: compound 26 ( $\beta$-phellandrene), compound 141 (flavone), compound 4 (4-cyclopentene-1,3-dione), compound 105 (ledene), compound 113 (unknown), compound 95 ( $\beta$-caryophyllene), compound 46 ( $\mathrm{N}$-methyl- $\mathrm{N}$-nitroso-2-propanamine), compound 20 ( $2 \mathrm{H}$ pyran-2,6(3H)-dione) and compound 47 (unknown). In order of correlation based on $\beta$ coefficiency, the four significantly negatively correlated compounds were compounds 52, 61 , 131 and 140 , none of which could be annotated based on $\geq 85 \%$ similarity with fragmentation patterns in the NIST11 database. The results of these statistical correlations with two factors were validated using cross-validation in the PLSR model. The model generates a validation rate for each factor, wherein validation for the model factors was high; for the first factor $\left(r^{2}=\right.$ $25.1 \%)$ and this was further increased with the second factor $\left(r^{2}=35.1 \%\right)$, results that are 
consistent with previous reports of two factors that reliably correlated biological activity and metabolic profile (Song, et al. 2014).

A

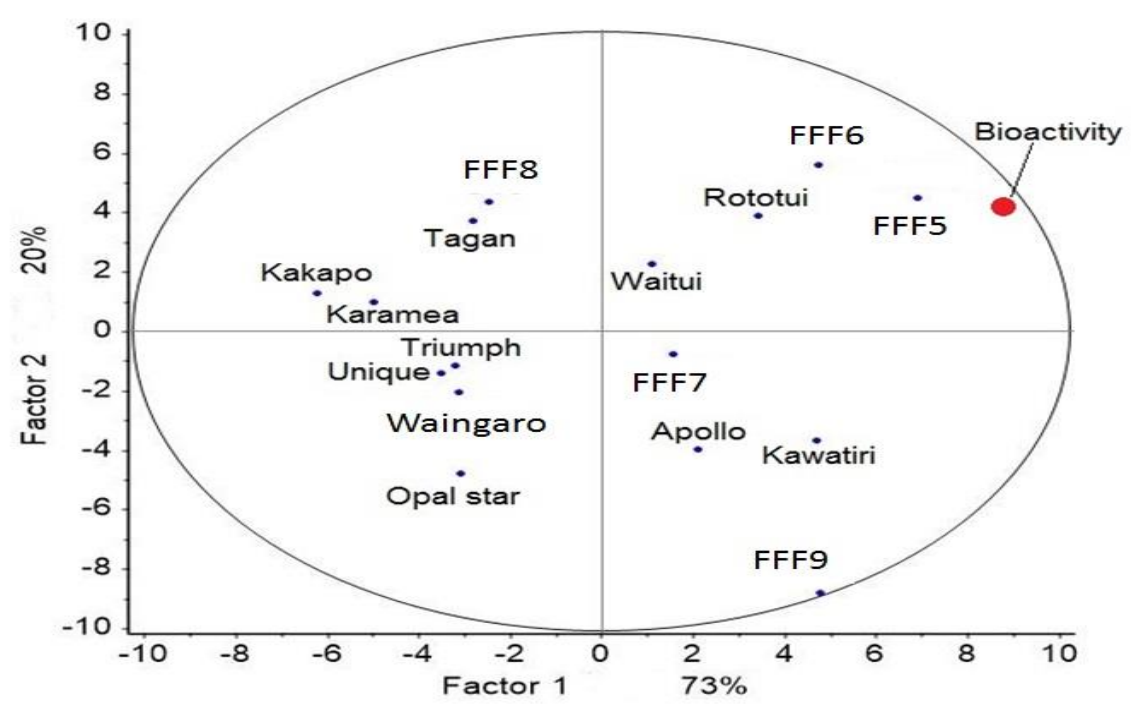

B

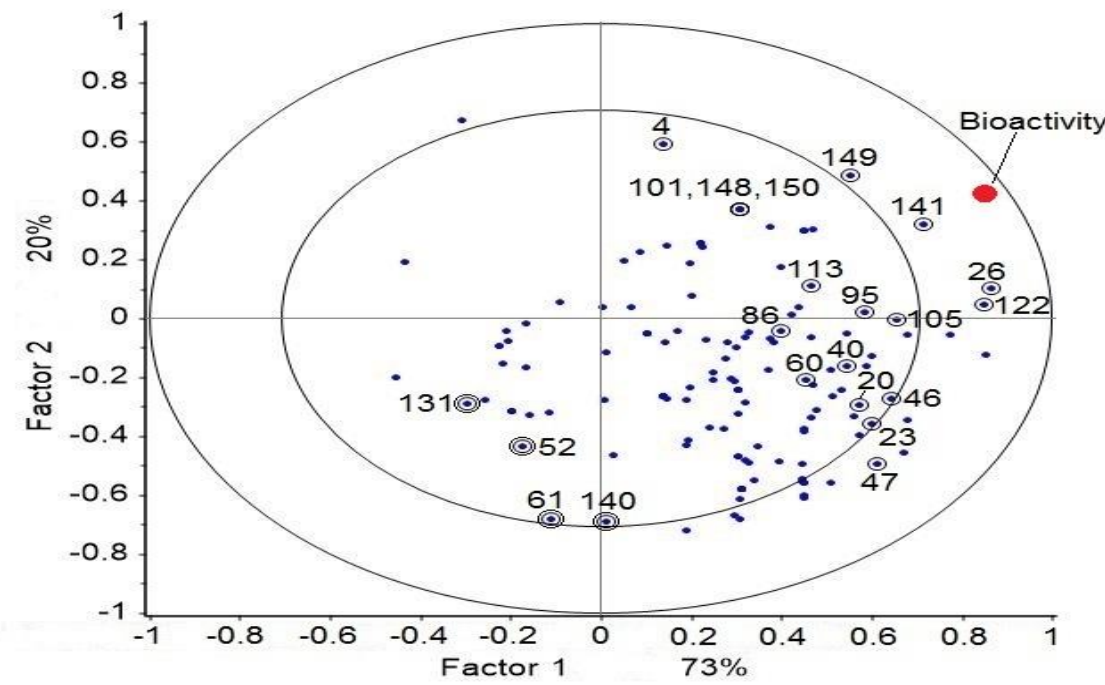

Figure 2-4. Partial least squares regression of (A) feijoa cultivar relative to bioactivity and (B) feijoa compound relative to bioactivity. Bioactivity (large red dot) is defined by growth inhibition of $S$. cerevisiae wherein the growth percentage inhibition was calculated via absorbance $\left(\mathrm{OD}_{600}\right)$ at mid-log in treated cells relative to untreated cells. Statistically significant compounds that are positively (one circle; numbers to the right side of the vertical line) or negatively (two circles; numbers to the left side of the vertical line) correlated with bioactivity are shown in (B). 
Of the nine significantly positively correlated compounds tentatively identified on the basis of $>85 \%$ similarity to the NIST database, three were commercially available (flavone, 4 cyclopentene-1,3-dione, and $\beta$-caryophyllene). We obtained the GC-MS fragmentation patterns and retention times of the authentic standards for these three compounds and compared these profiles with the profiles of the tentatively identified compounds detected in the feijoa cultivars (Table 2.2A). In each case the fragmentation patterns and retention times were identical, confirming the identity of these three compounds.

Table 2-2. Metabolites of feijoa extracts positively correlated with antifungal activity. Compounds identified in more than one feijoa cultivar (A) and compounds identified only in the FFF5 feijoa cultivar (B) positively correlated with bioactivity in $S$. cerevisiae based on PLSR statistical analysis. $\beta$-C, $\beta$-coefficiency correlating compound and bioactivity; $P$ value, Student's $t$-test comparing compound and bioactivity. Compounds were identified using (a) mass spectral and retention time match with an authentic standard, (b) mass spectral and LRI match, (c) mass spectral match only, or (d) unknown.

(A)

\begin{tabular}{lllllc}
\hline Compound & Compound & $\boldsymbol{\beta}$-C & $\boldsymbol{P}$ value & SI & Identity \\
\hline 26 & $\beta$-Phellandrene & 1.32 & 0.0008 & 94 & $\mathrm{c}$ \\
141 & Flavone & 1.31 & 0.0033 & 98 & $\mathrm{a}$ \\
4 & 4-Cyclopentene-1,3-dione & 1.24 & 0.0011 & 95 & $\mathrm{a}$ \\
105 & Ledene & 1.03 & 0.0012 & 90 & $\mathrm{~b}$ \\
113 & Unknown & 0.96 & 0.0146 & 88 & $\mathrm{~d}$ \\
95 & $\beta$-Caryophyllene & 0.92 & 0.0371 & 95 & $\mathrm{a}$ \\
46 & 2-Propanamine-N-methyl-N- & 0.75 & 0.0175 & 85 & $\mathrm{~d}$ \\
20 & nitroso- & & & $\mathrm{c}$ \\
47 & 2H-pyran-2,6(3H)-dione & 0.47 & 0.0349 & 92 & $\mathrm{~d}$
\end{tabular}

(B)

\begin{tabular}{lllllc}
\hline Compound & Compound & $\boldsymbol{\beta}-\mathbf{C}$ & $\boldsymbol{P}$ value & SI & Identity \\
\hline $\mathbf{5 8}$ & $(E)$-3-Hexenyl butyrate & 0.84 & 0.0763 & 89 & $\mathrm{a}$ \\
\hline
\end{tabular}




\subsubsection{Unique compounds in novel feijoa cultivars}

To follow up on our identification of the FFF5 and FFF6 cultivars as having the greatest bioactivity (Figure. 2.2), we investigated compounds with $>85 \%$ similarity to database matches that were identified only in these cultivars. We therefore examined the GC-MS analyses of these cultivars and detected $(E)$-3-hexenyl butyrate and two unknown compounds unique to the FFF5 cultivar (Table 2.2B), while there were no unique compounds detected in FFF6. We then compared the GC-MS fragmentation patterns and retention times of our tentatively identified (E)-3-hexenyl butyrate from the FFF5 cultivar with those of an authentic standards, which were the same as those of the standards, confirming the identity of this compound as being present only in the novel FFF5 feijoa cultivar.

\subsubsection{Confirmation of compound bioactivity}

To confirm the prediction of our multivariate analyses that the definitively identified flavone, $\beta$-caryophyllene and 4-cyclopenten-1-3-dione were positively correlated with growth inhibition of $S$. cerevisiae, we measured growth inhibition of $S$. cerevisiae in the presence and absence of standards. Relative to untreated cells, the $\mathrm{IC}_{50}$ values for the three compounds were $2.3 \mu \mathrm{M}, 35.8 \mu \mathrm{M}$ and 1.3 $\mu \mathrm{M}$ for flavone, -caryophyllene, and 4-cyclopenten-1-3-dione, respectively (Table 2.3). The unique compound observed only in the FFF5 cultivar, $(E)-3$ hexenyl butyrate, conferred growth inhibition via an $\mathrm{IC}_{50}$ of $22.7 \mu \mathrm{M}$ (Table 2.3). These results

validate our multivariate analysis-based correlation strategy, showing that positively correlated compounds predicted by the PLSR analysis are indeed bioactive in yeast cells. 

pathogens

Candida species commonly cause fungal infections and fatalities in immunocompromised hosts (Perfect 2017). To determine whether the compounds we identified as being inhibitory to growth of the saprobic yeast S. cerevisiae were more widely effective against human pathogenic fungal species, we quantified growth of four Candida species $(C$. albicans, C. glabrata, C. parapsilosis, and C. tropicalis) in the presence of three compounds that were positively correlated with bioactivity (flavone, $\beta$-caryophyllene, 4-cyclopentene-1-3dione) and one compound that was only identified in the FFF5 cultivar ((E)-3-hexenyl butyrate). Relative to untreated cells, the $\mathrm{IC}_{50}$ values for the four compounds were 1.5-2.6 $\mu \mathrm{M}$ for flavone, 1.5-2.3 $\mu \mathrm{M}$ for 4-cyclopentene-1-3-dione, 35.8-75.2 $\mu \mathrm{M}$ for $\beta$-caryophyllene, and 16.0-38.2 $\mu \mathrm{M}$ for 3-hexenyl butyrate (Table 2.4). Overall, the bioactivity of these four compounds against human pathogenic Candida species was consistent with bioactivity against $S$. cerevisiae (Table 2.4), providing further validation of our use of $S$. cerevisiae as a model fungi to study the antifungal bioactivity of feijoa cultivars. 
Table 2-3. The antifungal activity of methanolic extracts $(0.01 \% \mathrm{v} / \mathrm{v})$ of 16 feijoa cultivars against saprobic $S$. cerevisiae and pathogenic Candida species. The growth inhibition (\%) was calculated via absorbance $\left(\mathrm{OD}_{600}\right)$ at mid-log in treated cells relative to untreated cells. Data shown as mean \pm S.D. ${ }^{*}, p<0.05$, Student's $t$-test comparing treated and untreated cells.

\begin{tabular}{|c|c|c|c|c|c|}
\hline \multirow[b]{2}{*}{ Cultivar } & \multirow[b]{2}{*}{$\begin{array}{l}S . \\
\text { cerevisiae }\end{array}$} & \multicolumn{2}{|c|}{ Growth inhibition (\%) } & \multirow[b]{2}{*}{$\begin{array}{l}\text { C. } \\
\text { parapsilosis }\end{array}$} & \multirow[b]{2}{*}{$\begin{array}{l}\text { C. } \\
\text { tropicalis }\end{array}$} \\
\hline & & $\begin{array}{l}\text { C. } \\
\text { albicans }\end{array}$ & $\begin{array}{l}\text { C. } \\
\text { glabrata }\end{array}$ & & \\
\hline Waitui & $70.2 \pm 0.03 *$ & $52.7 \pm 0.01 *$ & $70.6 \pm 0.01 *$ & $58.7 \pm 0.03 *$ & $59.9 \pm 0.02 *$ \\
\hline R263 & $65.1 \pm 0.01 *$ & $62.1 \pm 0.05^{*}$ & $67.0 \pm 0.04 *$ & $57.8 \pm 0.01 *$ & $55.8 \pm 0.03 *$ \\
\hline FFF8 & $50.0 \pm 0.03 *$ & $39.3 \pm 0.01 *$ & $52.3 \pm 0.03 *$ & $34.4 \pm 0.02 *$ & $54.6 \pm 0.04 *$ \\
\hline FFF7 & $54.8 \pm 0.01 *$ & $43.8 \pm 0.03 *$ & $37.4 \pm 0.04 *$ & $58.7 \pm 0.03 *$ & $51.9 \pm 0.05 *$ \\
\hline Apollo & $44.0 \pm 0.02 *$ & $39.9 \pm 0.01 *$ & $54.2 \pm 0.02 *$ & $41.7 \pm 0.04 *$ & $39.8 \pm 0.02 *$ \\
\hline Kawatiri & $58.4 \pm 0.01 *$ & $58.4 \pm 0.05^{*}$ & $64.6 \pm 0.01 *$ & $60.9 \pm 0.01 *$ & $45.3 \pm 0.04 *$ \\
\hline Unique & $22.3 \pm 0.04 *$ & $24.9 \pm 0.03 *$ & $43.1 \pm 0.04 *$ & $34.9 \pm 0.05^{*}$ & $31.9 \pm 0.03 *$ \\
\hline Wangario & $30.1 \pm 0.03 *$ & $30.6 \pm 0.01 *$ & $59.7 \pm 0.03 *$ & $31.4 \pm 0.02 *$ & $36.1 \pm 0.04 *$ \\
\hline FFF5 & $85.2 \pm 0.01 *$ & $74.0 \pm 0.03 *$ & $87.3 \pm 0.01 *$ & $74.5 \pm 0.03 *$ & $69.3 \pm 0.01 *$ \\
\hline Tagan & $55.3 \pm 0.02 *$ & $48.0 \pm 0.01 *$ & $56.9 \pm 0.05^{*}$ & $39.8 \pm 0.02 *$ & $47.6 \pm 0.05 *$ \\
\hline FFF6 & $82.9 \pm 0.01 *$ & $78.0 \pm 0.01 *$ & $84.9 \pm 0.02 *$ & $69.7 \pm 0.02 *$ & $65.0 \pm 0.03 *$ \\
\hline Karamea & $30.6 \pm 0.03 *$ & $27.7 \pm 0.02 *$ & $49.0 \pm 0.03 *$ & $26.1 \pm 0.02 *$ & $50.7 \pm 0.05^{*}$ \\
\hline Opal star & $26.5 \pm 0.02 *$ & $19.3 \pm 0.03 *$ & $46.7 \pm 0.05^{*}$ & $34.2 \pm 0.01 *$ & $49.4 \pm 0.04 *$ \\
\hline Kakapo & $18.6 \pm 0.05^{*}$ & $12.2 \pm 0.04 *$ & $19.1 \pm 0.02 *$ & $38.8 \pm 0.03 *$ & $30.7 \pm 0.01 *$ \\
\hline Triumph & $32.9 \pm 0.03 *$ & $31.4 \pm 0.05^{*}$ & $51.5 \pm 0.04 *$ & $37.5 \pm 0.01 *$ & $47.2 \pm 0.03 *$ \\
\hline FFF9 & $55.5 \pm 0.01 *$ & $52.8 \pm 0.02 *$ & $69.0 \pm 0.01 *$ & $53.7 \pm 0.02 *$ & $51.5 \pm 0.04 *$ \\
\hline
\end{tabular}

Table 2-4. Half maximal inhibitory concentration $\left(\mathrm{IC}_{50}\right)$ of the authentic standards against saprobic S. cerevisiae and pathogenic Candida species. The concentration $(\mu \mathrm{M})$ that resulted in $50 \%$ growth inhibition was calculated using measurements of absorbance $\left(\mathrm{OD}_{600}\right)$ at mid-log in treated cells relative to untreated cells. Data shown as mean \pm S.D. Compounds are listed in order of $\beta$-coefficiency values (expected correlations with bioactivity based on the PLSR analysis).

\begin{tabular}{llllll}
\hline & \multicolumn{5}{c}{$\mathbf{I C}_{50}(\mu \mathrm{M})$} \\
\cline { 2 - 6 } Compound & $\begin{array}{l}\text { S. } \\
\text { cerevisiae }\end{array}$ & $\begin{array}{l}\text { C. } \\
\text { albicans }\end{array}$ & $\begin{array}{l}\text { C. } \\
\text { glabrata }\end{array}$ & $\begin{array}{l}\text { C. } \\
\text { parapsilosis }\end{array}$ & $\begin{array}{l}\text { C. } \\
\text { tropicalis }\end{array}$ \\
\hline Flavone & $2.3 \pm 0.5$ & $2.1 \pm 0.6$ & $2.6 \pm 0.3$ & $1.5 \pm 0.8$ & $1.8 \pm 0.9$ \\
$\begin{array}{l}\text { 4-Cyclopentene-1,3- } \\
\text { dione }\end{array}$ & $1.3 \pm 0.2$ & $2.3 \pm 0.4$ & $2.1 \pm 0.5$ & $2.0 \pm 0.2$ & $1.5 \pm 0.3$ \\
$\begin{array}{l}\text { B-Caryophyllene } \\
\text { 3-Hexenyl butyrate }\end{array}$ & $35.8 \pm 1.1$ & $53.0 \pm 0.9$ & $42.5 \pm 2.1$ & $42.5 \pm 1.4$ & $75.2 \pm 1.2$ \\
\hline
\end{tabular}


As these four compounds are merely a fraction of the 151 compounds that we identified in the 16 feijoa cultivars, we sought to further understand the antifungal potency of the total methanolic extracts derived from these cultivars, testing their abilities to inhibit the growth of C. albicans, C. glabrata, C. parapsilosis, and C. tropicalis. At $0.01 \% \mathrm{v} / \mathrm{v}$, the 16 cultivar extracts significantly inhibited growth of the four Candida species by $12-87 \%$ compared to untreated cells. Specifically, the FFF5 and FFF6 cultivar extracts inhibited growth of the pathogenic Candida strains by $69-85 \%$ compared to untreated cells (Table 2.3), consistent with these cultivar extracts being the most bioactive against $S$. cerevisiae (Figure. 2.2).

\subsubsection{Antifungal mechanism of action of 4-cyclopentene-1-3-dione}

Based on potent antifungal activity $\left(\mathrm{IC}_{50}=1-2 \mu \mathrm{M}\right)$, 4-cyclopentene-1,3-dione has the potential to be a lead for developement as a clinical antifungal drug once a mechanism of action is determined. Haploinsufficiency (hypersensitivity) of a heterozygous diploid yeast strain indicates the mutant gene is the drug target based on inhibition of the product of a single gene copy by the drug (Lee, et al. 2014). To determine if the target of 4-cyclopentene-1,3-dione is a known antifungal drug target, we evaluated sensitivity of heterozygous mutants bearing deletions of known antifungal drug targets. We observed that heterozygosity of the chitin synthase-1 and chitin synthase-2 genes (chs1 $1 / C H S 1$ and $c h s 2 \Delta / C H S 2)$ conferred significant hypersensitivity to 4-cyclopentene-1,3-dione with $70 \%$ and $74 \%$ growth reductions, respectively, compared to $10 \%$ growth reduction seen in the diploid WT strain (BY4743) (Figure. 2.5A). For comparison, other antifungal drug targets were not hypersensitive to 4cyclopentene-1,3-dione including the cell wall 1,3- $\beta$-D-glucan synthase FKS1 gene (target of caspofungin), the ergosterol biosynthesis ERG11 gene (target of ketoconazole), the sphingolipid synthesis LCB1 gene (target of myriocin), ergosterol biosynthesis HMG1 gene 
(target of atorvastatin), the cytosine deaminase FCY1 gene (resistance to flucytosine) and the RNAse subunits POP1 gene (hypersensitive to nystatin) (Figure. 2.5A). Interestingly, there was significant, albeit slight (21\%), inhibition of the hmg1 $/$ /HMG1 strain by 4-cyclopentene-1,3dione, though not to the same extent as chs1 $1 /$ CHS1. The CHS1 and CHS2 genes are the target of polyoxin (Cabib 1991), and the hypersensitivity of the chs $14 / C H S 1$ and chs $24 / C H S 2$ strains to 4 -cyclopentene-1,3-dione mirrored a $76 \%$ and $65 \%$ growth reduction in these strains by polyoxin (Figure. 2.5B).

A

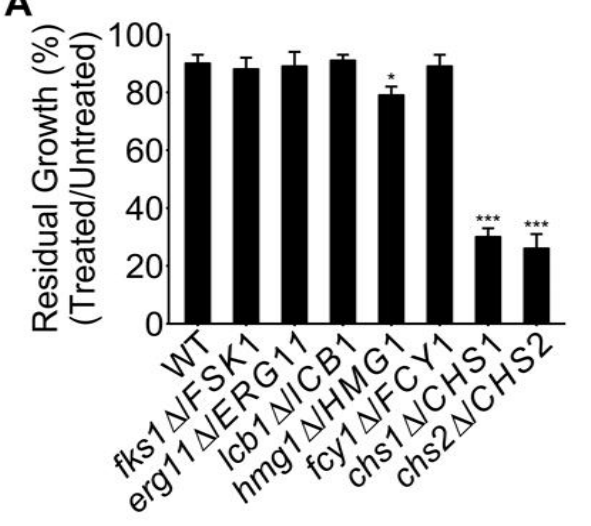

C

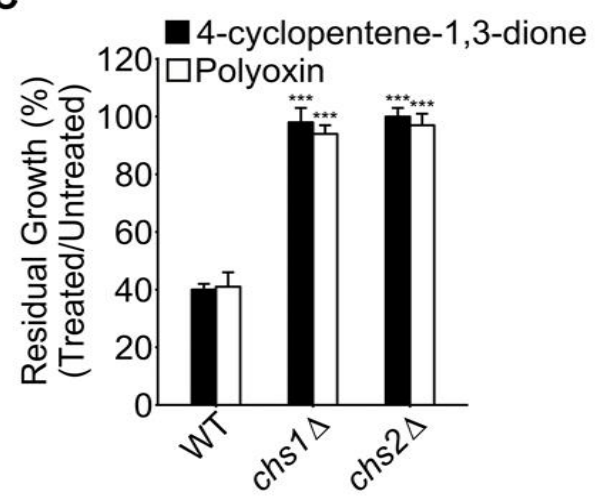

B

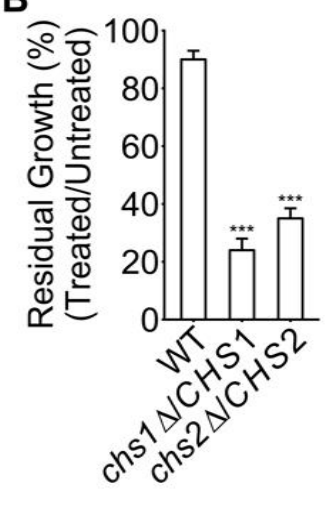

D

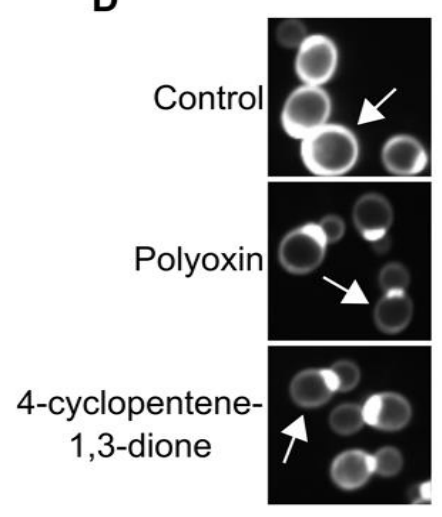

Figure 2 5. Figure 4. 4-cyclopentene-1,3-dione inhibits chitin synthesis via CHS1 and CHS2. A) Heterozygous deletion strains of known drug targets were incubated to mid-log and growth was measured at $\mathrm{OD}_{600}{ }^{*}, p<0.05$, Student's $t$-test comparing WT and mutant. B) Heterozygous chitin synthase deletion mutants were incubated to mid-log and growth was measured at $\mathrm{OD}_{600}{ }^{*}, p<0.05$, Student's $t$-test comparing WT and mutant. C) Haploid deletion mutants were incubated to mid-log and growth was measured at $\mathrm{OD}_{600}{ }^{*}, p<0.05$, Student's $t$-test comparing WT and mutant. D) Haploid WT cells were incubated to mid-log and chitin was stained with calcofluor white. Arrows shown to indicate septum. 
If a drug target is absent, cells will be resistant to that drug (Lee, et al. 2014). Consistent with CHS1 and CHS2 being the target of 4-cyclopentene-1,3-dione, chs1A and chs $2 \Delta$ haploid mutants were resistant to both 4-cyclopentene-1,3-dione and polyoxin, which induced no growth inhibition in chs $1 \Delta$ and $c h s 2 \Delta$ compared to $59 \%$ growth inhibition for each compound in the WT strain (Figure. 2.5C). To further characterize chitin synthesis inhibition, we examined the abundance of chitin in the cell wall. Using a fluorescent stain specific for chitin (calcafluor white) (Pringle 1991), both 4-cyclopentene-1,3-dione and polyoxin B reduced abundance of chitin in the septum (Figure. 2.5D), a morphology that is consistent with our demonstrated inhibition of the CHS1 and CHS2 gene products, which are the major regulators of chitin distribution in septa (M Chaudhary, et al. 2013). Together, these results indicate 4cyclopentene-1,3-dione and polyoxin share a common mechanism of action by targeting the the major chitin synthesis genes CHS1 and CHS2 and inhibiting the synthesis of chitin, a major structural component of the fungal cell wall that does not exist in mammalian cells and is critical to growth of all fungi. This specificity for chitin was further supported with an absence of antibacterial activity of 4-cyclopentene-1,3-dione against four bacterial species at $1.3 \mu \mathrm{M}$, the concentration that achieved $\mathrm{IC}_{50}$ antifungal activity. Consequently, 4-cyclopentene-1,3-dione was weakly antibacterial with $\mathrm{IC}_{50}$ values of $85 \pm 11,60 \pm 1,33 \pm 2$ and $105 \pm 14 \mu \mathrm{M}$ against $B$. subtilis (Gram positive lab strain), E. coli (Gram negative lab strain), S. aureus (pathogenic strain) and K. pneumoniae (pathogenic strain), respectively.

\subsection{DISCUSSION}

In this study, we investigated agronomically diverse feijoa cultivars to identify antifungal compounds with pharmaceutical potential. These compounds were sourced from feijoa peel waste to provide the first explicit attempt to add high value to the peels that are 
currently discarded by the ton in the feijoa juicing industry. We investigated 16 feijoa cultivars, both commercial and non-commercial within a feijoa breeding program, and in an unbiased fashion correlated antifungal bioactivity with the observed metabolite content (151 volatile compounds) rather than in a traditional reductionist fashion of bioassay-guided fractionation of a subset of these compounds. Using established methodology to link metabolomic and bioassay results (Coutinho, et al. 2016), our multivariate analysis demonstrated strong correlation between antifungal bioactivity and 22 compounds present in methanolic extracts of feijoa fruit peels. One of these compounds, 4-cyclopentene-1,3-dione, was potently antifungal against Candida species that cause the most fungal infections in humans, including C. albicans, via a fungal-specific mechanism targeting chitin in fungal cell walls. As no chitin synthesis inhibitor has been clinically approved for use in humans, our results indicate 4-cyclopentene-1,3-dione has such clinical potential, particularly since no cyclopentenedione reported to date has demonstrated inhibition of pathogenic Candida species via a chitin synthesis mechanism.

In addition to recovering compounds detected in previous studies such as flavone, $\beta$ caryophyllene (Fernandez, et al. 2004; Weston 2010), 3-octanone (Fernandez, et al. 2004), trans-calamenene (Fernandez, et al. 2004) and globulol (Binder and Flath 1989), we also detected compounds that have not been reported from feijoa to date, although these are primarily tentative annotations and not formal positive identifications. In one case of a positive identification, we provide the first report of 4-cyclopentene-1,3-dione being present in feijoa. We confirmed the compound identity via GC-MS analysis of an authentic standard, and demonstrated potent antifungal activity via growth inhibition of multiple pathogenic Candida species. The presence of 4-cyclopentene-1,3-dione in a plant species is not specific to feijoa as this compound has previously been identified in the Chinese nutmeg tree Torreya grandis (Yao, et al. 2014). Interestingly, the most potent FFF5 cultivar contained relatively the most 4cyclopentene-1,3-dione among the 16 cultivars, raising the possibility that the antifungal potency of FFF5 is largely a consequence of 4-cyclopentene-1,3-dione itself or enhanced 4- 
cyclopentene-1,3-dione bioactivity due to synergy with other bioactive compounds (e.g., butyrate compounds can increase the bioactivity of other volatile organic compounds (Strobel, et al. 2008)).

In regards to the potency of antifungal bioactivity, 4-cyclopentene-1,3-dione is comparable with established antifungal drugs. Clinically used drugs from the polyene group such as simvastatin and atorvastatin exhibit $\mathrm{IC}_{50}$ values of $3 \mu \mathrm{M}$ and $10 \mu \mathrm{M}$, respectively, against Candida albicans (Macreadie, et al. 2006), while azole drugs used in the clinic such as fluconazole have $\mathrm{IC}_{50}$ values of $\sim 3 \mu \mathrm{M}$ against different Candida species (Wardle, et al. 1995). Our study determined that 4-cyclopentene-1,3-dione exhibits $\mathrm{IC}_{50}$ values of 1.5-2.3 $\mu \mathrm{M}$ against four Candida species, indicating that this compound is as potent as antifungal drugs used in the clinic today. Although the antifungal activity of 4-cyclopentene-1,3-dione has not previously been demonstrated, it is consistent with antifungal activity for structurally related coruscanone A ( $\mathrm{Li}$, et al. 2004). Coruscanone A and a desmethoxy derivative were each comparable to the clinically approved antifungals fluconazole and amphotericin B with $\mathrm{IC}_{50}$ values of $0.5-3 \mu \mathrm{M}$ against Candida species . While the antifungal mechanism of coruscanone $\mathrm{A}$ is not yet resolved, our results show that 4-cyclopentene-1,3-dione is antifungal via chitin synthesis inhibition.

As chitin is a major component of fungal cell walls that is missing in vertebrate organisms, chitin biosynthesis is a specific and promising target to treat fungal infections without inhibiting those same pathways in human hosts (M Chaudhary, et al. 2013; Perfect 2017). Nikkomycin and polyoxin are two established chitin synthase inhibitors that were isolated more than 40 years ago from cultures of Streptomyces species. While both are pyrimidine nucleoside peptides, neither has been clinically approved as a pharmaceutical drug, albeit polyoxin is an active ingredient in commercial fungicides in the agricultural and horticultural industries (M Chaudhary, et al. 2013). Both nikkomycin and polyoxin inhibit CHS1 and CHS2, which is consistent with our results comparing 4-cyclopentene-1,3-dione and 
polyoxin. Although these three compounds share a mechanism, it is plausible that 4cyclopentene-1,3-dione will exhibit different pharmacokinetics than nikkomycin and polyoxin given their completely different structural backbones. For example, the more lipophillic cyclopentenedione backbone will exhibit vastly different solubility than pyrimidine nucleoside peptides, and also will be less susceptible to hydrolysis. Given the Michael acceptor and acidic 1,3-diketo functional groups within the compact cycopentenedione scaffold, covalent inhibition of targets is also plausible. In addition to nikkomycin and polyoxin isolated from bacteria, there have been other structurally diverse natural product chitin synthesis inhibitors with antifungal activity, notably five compounds isolated from four fungal species and 14 compounds isolated from 10 plant species (Li, et al. 2004; M Chaudhary, et al. 2013). With our results, 4cyclopentene-1,3-dione can be added to this list of natural chitin synthase inhibitors.

Cyclopentenedione compounds are a relatively new group of $\sim 100$ secondary metabolites isolated primarily from plants as well as fungi and bacteria (Sevcikova, et al. 2014). It is plausible that other cyclopentenedione compounds, in addition to our demonstration for 4cyclopentene-1,3-dione, are antifungal by a common mechanism of chitin synthesis inhibition. For example, the cyclopentenediones linderone and methyllinderone exhibited antifungal activity against plant pathogens via chitin synthase inhibition (Hwang, et al. 2007). However, these cyclopentenediones did not inhibit CHS1 activity, which was the main reason these compounds did not effectively kill Candida species ( $\mathrm{IC}_{50}>700 \mu \mathrm{M}$ ). In contrast, we demonstrated that 4-cyclopentene-1,3-dione inhibited CHS1 and thus inhibited growth of four Candida species with $\mathrm{IC}_{50} \sim 1-2 \mu \mathrm{M}$. More broadly than specificity for chitin synthase genes, the reports of anti-cancer and anti-inflammatory bioactivities among cyclopentenedione compounds in mammalian cells suggests that chitin synthesis inhibition is not the only bioactivity mechanism in this family of compounds since mammalian cells do not contain chitin (Sevcikova, et al. 2014). A similar phenomenon was evident in this study, with 4-cyclopentene- 
1,3-dione exhibiting low level antibacterial activity relative to the potent antifungal activity (consistent with bacteria not containing chitin).

Recognizing the potential use of cyclopentenedione compounds as novel pharmaceutical drugs, a library of compounds derived from a parental cyclopentenedione compound was generated and chemically characterized but not characterized for any biological activity (Papouskova, et al. 2016). Our finding of potent antifungal activity of 4-cyclopentene1,3-dione as a chitin synthesis inhibitor clearly warrants further evaluation of structure-activity relationships. Similar medicinal chemistry approaches have been used to enhance the antiinflammatory and anti-cancer activities of compounds with a cyclopentenedione backbone (Sevcikova, et al. 2014). As the cyclopentenedione backbone is highly reactive, it has a high potential as a lead for commercial synthesis and testing of analogues to probe antifungal structure-activity relationships.

In conclusion, we conducted the first systematic evaluation of bioactivity across feijoa cultivars and demonstrated there is enormous functional chemical diversity among feijoa cultivars to exploit as a natural source of bioactive compounds from agricultural waste. We used bioactivity-guided metabolite profiling of peels derived from 16 commercial and experimental feijoa cultivars to correlate 151 volatile compounds with antifungal activity. These compounds were identified via unbiased metabolite profiling of both novel, noncommercial and commercially available feijoa cultivars, which collectively indicate that there is extensive chemical diversity within closely related feijoa cultivars. All cultivars were substantially antifungal at $0.01 \%$ (v/v) based on growth inhibition of Saccharomyces and Candida species. The two experimental cultivars FFF5 and FFF6 that exhibited the most antifungal activity contained the compound 4-cyclopentene-1,3-dione, which we showed to be potently antifungal against Saccharomyces and Candida species. It will be necessary to measure the abundance of 4-cyclopentene-1,3-dione in each feijoa peel to precisely determine how much 
of this potent antifungal compound can be isolated from a given amount of feijoa peel waste. Via our identification of the first cyclopentenedione compound to potently kill Candida species via a fungal-specific mechanism of chitin synthesis inhibition, there is potential of a high value antifungal pharmaceutical in a waste product in the feijoa juicing industry, which warrants further investigation of volatile and non-volatile compounds for more activities in addition to antifungal activity. 


\section{Chapter 3 Elucidating the antifungal mechanism of action of EtOH- vescalagin}

\subsection{INTRODUCTION}

Fungal infections result in significant mortality and cost around the world annually (Arif, et al. 2009). There are currently five classes of antifungal drugs distinguished by the drug target (ergosterol inhibitor, cell wall inhibitor, sphingolipid inhibitor, nucleic acid inhibitor, protein inhibitor). However, increasing rates of antifungal resistance have led to a recent and ongoing priority to identify a novel target for antifungal drugs. Microbes have been the major source of antifungal drugs to date (Vicente, et al. 2003). Plants have been investigated as well for their secondary metabolites that can be bioactive against microorganisms.

Polyphenols are low molecular weight secondary metabolites produced by plants that are integral to plant defense systems against microbial pathogens. The four main groups of the highly bioactive polyphenols are phenolic acid, stilbenes, lignans, and flavonoids (Adlercreutz and Mazur 1997; Pandey and Rizvi 2009). Polyphenols have been reported with antioxidant (Daglia 2012; Rice-evans, et al. 1995; Scalbert, et al. 2005), antimicrobial (Daglia 2012), antifungal (Daglia 2012), anti-inflammatory (Yoon and Baek 2005) and anti-cancer (Fresco, et al. 2006; Fresco, et al. 2010) activities.

Ellagitannins are a specific type of polyphenol defined by hydrolysable tannins which are derivatives of ellagic acid found in fruits such as pomegranate, black raspberries and walnuts (Landete 2011). Vescalagin is a type of water-soluble ellagitannin that has been isolated from European oak heartwood (Vivas, et al. 2004). Vescalagin is clearly bioactive with previous anti-cancer (Auzanneau, et al. 2011), antibacterial (Becker, et al. 2005) and anti-diabetic (Huang, et al. 2016) activities. Notably, the antifungal bioactivity has been tested against 
Saccharomyces cerevisiae and Candida albicans, yet antifungal activity was not detected (Becker, et al. 2005).

Since the ethanol adduct form of vescalagin (EtOH-vescalagin) has previously been shown to exhibit structural activity distinct from the parent vescalagin compound (Puech et al 1999) and vescalagin showed no antifungal activity itself, we hypothesized that the ethanol adduct form of vescalagin exhibited antifungal activity in contrast to vescalagin. Here we investigated the antifungal activity and associated mechanism of action of feijoa-derived EtOHvescalagin in pathogenic Candida species as well as the genetic model S. cerevisiae. With nanomolar potency, EtOH-vescalagin inhibited the growth of all of these fungal species. Using the unbiased approach of chemical genomics, we determined the antifungal mechanism of action included iron depletion, zinc toxicity, and disrupted retromer recycling.

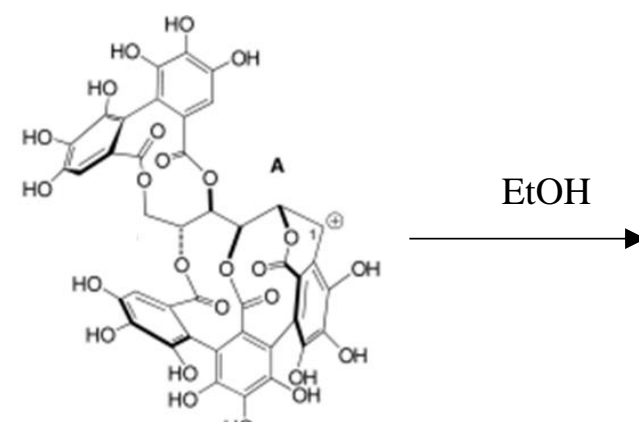

Vescalagin

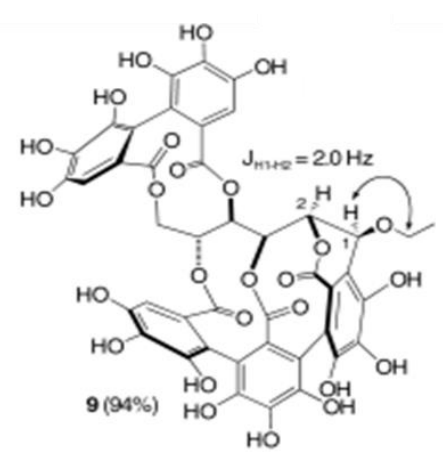

EtOH-vescalagin

Figure 3-1. Structures of vescalagin and EtOH-vescalagin. 


\subsection{METHODS}

\subsubsection{Feijoa compounds}

EtOH-vescalagin, castalagin, casurinin, flavone, pedunculagin and pentagalloyl glucose were isolated from feijoa and kindly provided by Dr Stephen Bloor (Callaghan Innovation).

\subsubsection{Chemicals}

Bathophenanthrolinedisulfonic acid disodium salt hydrate (BPS), N,N,N', $\mathrm{N}^{\prime}-$ Tetrakis(2-pyridylmethyl)ethylenediamine (TPEN), filipin, Zinquin ethyl ester ( 2-Methyl-8[(4-methylphenyl)sulfonylamino]-6-(ethyloxycarbonylmethyloxy)quinolone), Nile Red and DMSO were purchased from Sigma. FM4-64 was purchased from Life Technologies.

\subsubsection{Strains}

Table 3-1. Yeast strains used in this project

\begin{tabular}{|c|c|c|}
\hline Strain & Genotype & Reference \\
\hline $\begin{array}{l}\text { BY4741 } \\
\text { (haploid WT) }\end{array}$ & his $3 \Delta 1$ leu $2 \Delta 0$ met $15 \Delta 0$ ura $3 \Delta 0$ & $\begin{array}{l}\text { Open } \\
\text { Biosystems }\end{array}$ \\
\hline $\begin{array}{l}\text { BY4743 } \\
\text { (diploid WT) }\end{array}$ & $\begin{array}{l}\text { his } 3 \Delta 1 / \text { his } 3 \Delta 1 \text { leu } 2 \Delta 0 / \text { leu } 2 \Delta 0 \\
\text { lys } 2 \Delta 0 / \text { LYS2/MET15/met15 } 50 \text { ura } 3 \Delta 0 / \text { ura } 3 \Delta 0\end{array}$ & $\begin{array}{l}\text { Open } \\
\text { Biosystems }\end{array}$ \\
\hline CM100 (WT) & MATa can1-100 his3-11,15 leu2-3112 trp1-1 ura3-52 & $\begin{array}{l}\text { (MacDiarmid, } \\
\text { et al. 2000) }\end{array}$ \\
\hline CM137 & $\begin{array}{l}\text { MATa can1-100 his3-11,15 leu2-3112 trp1-1 ura3-53 } \\
\text { zrc1::HIS1 cot } 1:: \text { KANR }\end{array}$ & $\begin{array}{l}\text { (MacDiarmid, } \\
\text { et al. 2000) }\end{array}$ \\
\hline JSY12 & $\begin{array}{l}\text { MATa can1-100 his3-11,15 leu2-3112 trp1-1 ura3-54 } \\
\text { msc } 2:: \text { KANRzrg17::NAT1 }\end{array}$ & (Ellis 2004) \\
\hline JSY6 & $\begin{array}{l}\text { MATa can } 1-100 \text { his } 3-11,15 \text { leu2-3112 trpl-1 ura3-55 } \\
\text { zrc1::HIS3cot } 1:: U R A 3 m s c 2:: \text { KANRzrg } 17:: N A T 1\end{array}$ & (Ellis 2004) \\
\hline $\begin{array}{l}\text { WT UPRE-GFP } \\
\text { (YCG261) }\end{array}$ & 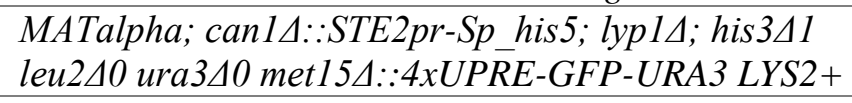 & $\begin{array}{l}\text { (Bircham, et } \\
\text { al. 2011) }\end{array}$ \\
\hline $\begin{array}{l}\text { C. albicans } \\
\text { ATCC } 10231\end{array}$ & & $\begin{array}{l}\text { (Curator } \\
\text { 2004) }\end{array}$ \\
\hline $\begin{array}{l}\text { C. parapsiliosis } \\
\text { ATCC } 90018\end{array}$ & & $\begin{array}{l}\text { (Curator } \\
\text { 2004) }\end{array}$ \\
\hline $\begin{array}{l}\text { C. glabrata } \\
\text { ATCC } 90030\end{array}$ & & $\begin{array}{l}\text { (Curator } \\
\text { 2004) }\end{array}$ \\
\hline $\begin{array}{l}\text { C. tropicalis } \\
\text { ATCC } 13803\end{array}$ & & $\begin{array}{l}\text { (Curator } \\
\text { 2004) }\end{array}$ \\
\hline
\end{tabular}




\subsubsection{Media}

All strains were grown in SC media buffered with 25 mM HEPES (hereafter referred to as SC) with dropout of specific amino acids as required for selection.

\subsubsection{Liquid-based assay of antifungal activity}

Growth of fungal strains was measured in biological and technical triplicate as described previously in a publication (Ruggles, et al. 2014) as well as this thesis (Chapter 2, Section 2.2.4).

\subsubsection{Genome-wide barcode sequencing analyses}

Sensitivity of homozygous and heterozygous diploid deletion mutant libraries to EtOH-vescalagin was evaluated using barcode sequencing as previously described (Robinson, et al. 2014). First, the diploid heterozygous deletion library (Open Biosystems) or the diploid homozygous deletion library (Open Biosystems) was incubated for $24 \mathrm{~h}$ at $30^{\circ} \mathrm{C}$ on YPD + G418 $(100 \mu \mathrm{g} / \mathrm{mL})$ agar plates in 384 colony format, and then colonies were pooled twice with $5 \mathrm{~mL}$ of YPD + G418 with a glass rod across the agar surface and collected in a sterile conical flask on ice. Glycerol was added to a final concentration of $15 \% \mathrm{v} / \mathrm{v}$ and cell density was calculated using a Coulter counter. Aliquots of each library were stored at $-80^{\circ} \mathrm{C}$ at $1 \times 10^{9}$ cells. A single aliquot $(1 \mathrm{~mL})$ of the same frozen stock of the diploid heterozygous or homozygous deletion library was then inoculated in $10 \mathrm{~mL} \mathrm{SC}$, incubated at $30^{\circ} \mathrm{C}$ for $18 \mathrm{~h}$, and this culture was used to inoculate $100 \mathrm{~mL} \mathrm{SC}$ at $5 \times 10^{6}$ cells $/ \mathrm{mL}$ that was further incubated at $30^{\circ} \mathrm{C}$ for 5 h. These cells in log phase were used to inoculate fresh $2 \mathrm{~mL} \mathrm{SC}$ in the presence and absence of $305 \mathrm{nM}$ EtOH-vescalagin to a final concentration of $5 \times 10^{6}$ cells $/ \mathrm{mL}$ (i.e., approximately 1000 representations of each strain) and incubated at $30^{\circ} \mathrm{C}$ for $15 \mathrm{~h}$ (the first 10 generations), which was measured at $\mathrm{OD}_{600}$ to confirm that cells were at $\log$ phase $\left(\mathrm{OD}_{600}=0.4-0.6\right)$ and to confirm EtOH-vescalagin reduced growth by $20 \%$ compared to untreated cells. Cells from the 
first 10 generations were then used to inoculate fresh $2 \mathrm{~mL} \mathrm{SC}$ in the presence and absence of $305 \mathrm{nM}$ EtOH-vescalagin to a final concentration of $5 \times 10^{6}$ cells $/ \mathrm{mL}$ and incubated at $30^{\circ} \mathrm{C}$ for $15 \mathrm{~h}$ (the second 10 generations).

Then genomic DNA (gDNA) was extracted using the YeaStar Genomic DNA Kit (Zymo Research) according to Protocol I in the manufacturer's instructions. In brief, $5 \times 10^{7}$ cells were harvested by centrifugation at $500 \mathrm{x} \mathrm{g}$ for $2 \mathrm{~min}$, the pellet was resuspended by vortexing in $120 \mu \mathrm{L}$ of $\mathrm{YD}$ digestion buffer and $5 \mu \mathrm{L}$ of R-Zymolase, and the tubes were incubated at $37^{\circ} \mathrm{C}$ for $40-60 \mathrm{~min}$. Then $120 \mu \mathrm{L}$ of lysis buffer was added, gently mixed by inversion, and $250 \mu \mathrm{L}$ of chloroform was added and mixed for $1 \mathrm{~min}$. Tubes were then centrifuged at 10,000 rpm for $2 \mathrm{~min}$, and the upper layer (gDNA) was transferred onto a Zymo spin III column. The liquid phase was first removed by centrifugation at 10,000 rpm for $1 \mathrm{~min}$, and $300 \mu \mathrm{L}$ of DNA wash buffer was added and removed twice by centrifugation at 10,000 rpm for $1 \mathrm{~min}$. The Zymo column was then transferred into a sterile $1.5 \mathrm{~mL}$ centrifuge tube and the gDNA was eluted with $60 \mu \mathrm{L}$ sterile $\mathrm{dH}_{2} \mathrm{O}$ onto the membrane that was incubated for $1 \mathrm{~min}$ at room temperature, and centrifuged at $10,000 \mathrm{rpm}$ for $10 \mathrm{~s}$ to yield $10-20 \mu \mathrm{g}$ purified gDNA. gDNA was used as template in a PCR using universal primers (Robinson, et al. 2014) to amplify the unique $20 \mathrm{bp}$ UPTAG (upstream) and $20 \mathrm{bp}$ DNTAG (downstream) barcodes representative of every gene deletion. Then $60 \mathrm{ng}$ of each UPTAG and DNTAG amplicon library pool separately underwent PCR amplification for the incorporation of the Illumina P5 adapter sequence, and finally the UPTAG and DNTAG libraries were combined at a 1:1 ratio and $50 \mathrm{bp}$ single end reads were sequenced on an Illumina flow cell using an IlluminaHiSeq 2000 platform. Read matching and bioinformatic analyses were conducted as previously described (Robinson, et al. 2014) to generate logFC (change in mutant barcode sequence abundance in treatment compared to control), FDR (false discovery rate) and logCPM (quality of barcode sequencing results) for every gene deletion in the heterozygous and homozygous diploid libraries. 
Validation of sensitive heterozygous or homozygous strains in the competitive pool format was evaluated via growth analyses of specific strains (in standard isolated format) using the liquid growth assay (Section 2.3.4). Selected strains were grown in 96-well plate in SC media at $30^{\circ} \mathrm{C}$ for overnight. This plate was used as a source plate to pin into the target 96 -well plate containing SC media with and without EtOH-vescalagin, and incubated at $30^{\circ} \mathrm{C}$ to midlog growth phase $\left(\mathrm{OD}_{600}=0.4\right.$ in untreated control $)$. The growth was measured via absorbance $\left(\mathrm{OD}_{600}\right)$ on an Envision plate reader (Perkin Elmer). Sensitive genes were analyzed for function using Gene Ontology (Ashburner, et al. 2000).

\subsubsection{Gene overexpression analysis}

Plasmids expressing specific genes under the control of the GAL1 promoter (Cooper, et al. 2006) were prepared using alkaline lysis purification (Sambrook and Russell 2006) and transformed in yeast strains using lithium acetate (Gietz and Schiestl 2007). Transformed strains were grown overnight in liquid SC $-\mathrm{Ura}+2 \%$ raffinose and then growth was compared using the liquid growth assay (Section 2.3.4) in SC $-\mathrm{Ura}+2 \%$ glucose relative to SC $-\mathrm{Ura}+$ $2 \%$ galactose $+1 \%$ raffinose

\subsubsection{Zincosome analysis}

Cytoplasmic zinc-containing vesicles (zincosomes) were visualized using the fluorescent stain Zinquin (Sigma) as previously described (Devirgiliis, et al. 2004) (Li and Kaplan 2001). Cells were grown overnight in SC media and then inoculated at OD $_{600}$ of 0.1 in SC media with and without EtOH-vescalagin. Cells at mid-log phase of growth (at $14 \mathrm{~h}$ ) were washed with PBS, fixed with $3.7 \%$ formaldehyde for $1 \mathrm{~h}$ at $30^{\circ} \mathrm{C}$, washed with $\mathrm{PBS}$, resuspended in $25 \mu \mathrm{M}$ Zinquin (in PBS), incubated for 30 min at RT, washed twice with PBS, and imaged using fluorescent microscopy (Olympus BX63). 


\section{Atomic Absorption Spectroscopy}

Intracellular levels of zinc and iron were quantified using atomic absorption spectroscopy (AAS) as previously described (Simm, et al. 2007). In brief, cells were grown to mid-log in $1 \mathrm{~mL} \mathrm{SC}$, centrifuged at 1000xg for $5 \mathrm{~min}$, washed twice in cold $0.5 \mathrm{mM}$ EDTA, washed in cold deionised water, digested in nitric acid $(40 \% \mathrm{v} / \mathrm{v})$ overnight at $90^{\circ} \mathrm{C}$ on a heat block, and measured with standards (Sigma) on an AAS (iCE3500) instrument fitted with a GFS35 graphite furnace unit (Thermo Fisher Scientific) using argon as the purge gas.

\subsubsection{Protein expression}

In the WT background as well as gene deletion backgrounds generated using SGA methodology (Boone, et al. 2007), abundance and localization of proteins was investigated in strains expressing a GFP-tagged protein of interest relative to internal RFP-tagged markers of the cytoplasm and nucleus (Bircham, et al. 2011). Cells were grown to mid-log in SC in 96well plates, transferred to a YPD agar plate in 384 format, and incubated at $30^{\circ} \mathrm{C}$ for $14 \mathrm{~h}$. Cells from this plate were then transferred to SC media with and without EtOH-vescalagin in a blackwalled, clear bottom 384 well plate (Perkin Elmer), incubated at $30^{\circ} \mathrm{C}$ for $4 \mathrm{~h}$, and visualized using Evo-Tec OPERA high throughput confocal microscope (Perkin Elmer) with an exposure time of $400 \mathrm{~ms}$ and 3 stacks per field. GFP excitation was at $488 \mathrm{nM}$ while RFP (mCherry and RedStar) excitation was at $561 \mathrm{nM}$ (Bircham, et al. 2011). GFP quantification was completed using ACAPELLA v2.0 (Perkin Elmer) that recognized cells by the RFP markers (i.e., the cytosol with RedStar and the nucleus with mCherry). GFP expression was normalized against RFP expression for each well, and the mean values for each triplicate were compared using statistical analyses. 


\subsubsection{Iron chelation assay}

Iron chelation assay was conducted with measurement of ferrous iron chelating activity as previously described (Dinis, et al. 1994; Robu, et al. 2012). Briefly, $20 \mu \mathrm{L}$ of $\mathrm{FeCl}_{2}$ was added to each well in a 96-well plate, followed with the addition of $40 \mu \mathrm{L}$ of $5 \mathrm{mM}$ Ferrozin (Sigma) and $100 \mu \mathrm{L}$ of compound. Then the mixture was incubated for $10 \mathrm{~min}$ at $\mathrm{RT}$, and the absorbance of each well was measured at $562 \mathrm{nM}$ on an Envision plate reader (Perkin Elmer). Chelation activity was calculated using the following formula:

$\%$ chelation activity $=100 *[(\mathrm{Ac}-\mathrm{As} / \mathrm{Ac})]$

where Ac is absorbance of control and As is absorbance of treatment (EtOHvescalagin).

\subsection{RESULTS}

\subsubsection{All feijoa polyphenols are bioactive in yeast}

To initially determine if a subset of polyphenols from feijoa fruit (EtOH-vescalagin, castalagin, pedunculagan pentagalloyl glucose, flavone, casurinin) are bioactive, we conducted the bioassay test on a wild-type S. cerevisiae strain (BY4741). Growth was measured in SC via absorbance $\left(\mathrm{OD}_{600}\right)$ and compared between treated and control cells. At $1 \mu \mathrm{M}$, all six polyphenol compounds inhibited growth and were bioactive (Figure 3.2). At 14 h, EtOHvescalagin showed highest activity with $82 \%$ inhibition, followed by castalagin and pentagalloyl glucose with $75 \%$ growth inhibition. Less potent were pedunculagin, flavone and casurinin with 65,47 and $32 \%$ inhibition, respectively. We thus chose to further investigate EtOH-vescalagin, the most potent compound. 
A
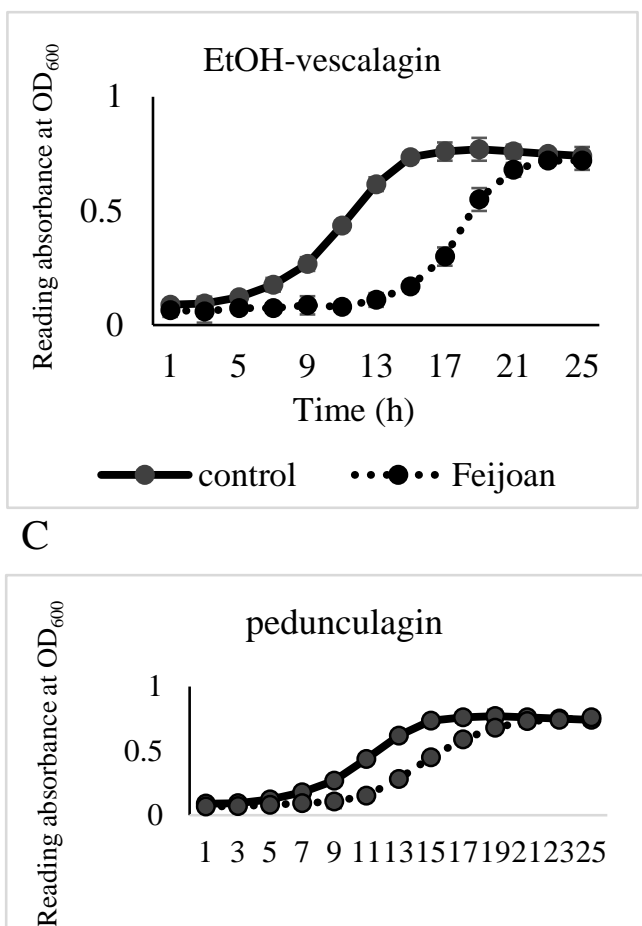

Time (h)

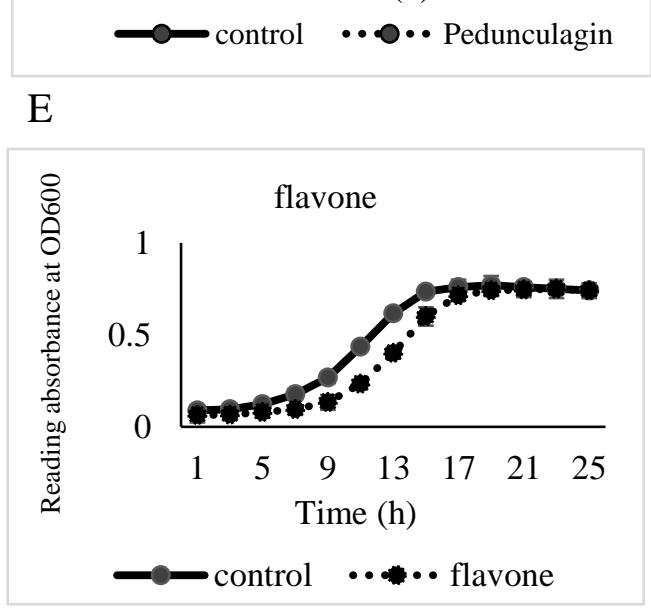

$\mathrm{B}$

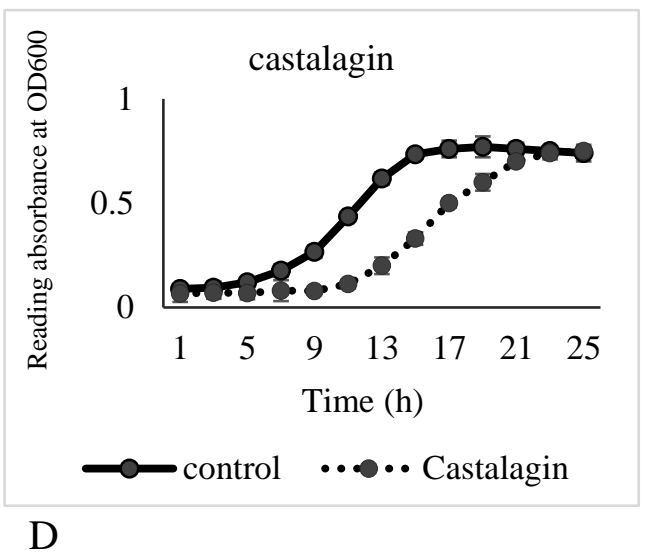

$\mathrm{D}$

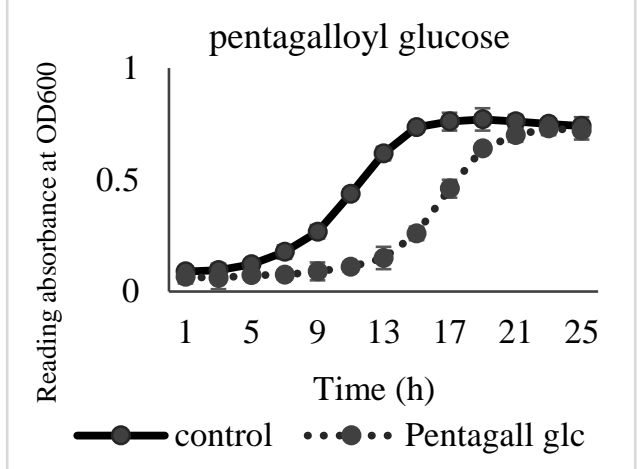

$\mathrm{F}$

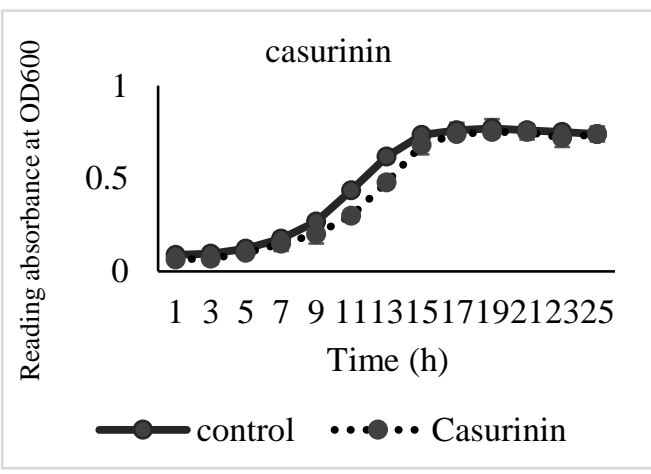

Figure 3-2. Polyphenols from feijoa are growth inhibitors in WT yeast at $1 \mu \mathrm{M}$. The growth was measured by reading absorbance at $\mathrm{OD}_{600}$ for 24 hours and compared to relative control. The values are triplicate average. A) EtOH-vescalagin. B) castalagin. C) pedunculagin. D) pentagalloyl glucose. E) flavone. F) casurinin.

\subsubsection{EtOH-vescalagin is antifungal against pathogenic Candida strains}

To further determine the antifungal activity of $\mathrm{EtOH}$-vescalagin, we quantified growth of four fungal human pathogenic species $C$. albicans, $C$. glabrata, $C$. parapsiliosis and $C$. 
tropicalis in the presence of different concentrations of EtOH-vescalagin. Growth was measured at mid-log phase after 14 hours. Relative to untreated cells, EtOH-vescalagin conferred a potent antifungal activity at nanomolar concentrations in all species (Table 3.2). EtOH-vescalagin was antifungal against $C$. tropicalis and $C$. glabrata with $\mathrm{IC}_{50}$ values of 551 $\mathrm{nM}$ and $521 \mathrm{nM}$, respectively. EtOH-vescalagin was also antifungal against $C$. albicans and $C$. parapsilosis with $\mathrm{IC}_{50}$ values of $395 \mathrm{nM}$ and $455 \mathrm{nM}$, respectively. These results demonstrate that Et-OH vescalagin exhibits potent antifungal activity against pathogenic Candida species, which is consistent with growth inhibition of S. cerevisiae.

Table 3-2. Half maximal inhibitory concentration $\left(\mathrm{IC}_{50}\right)$ of EtOH-vescalagin in pathogenic Candida species. Values are from triplicate \pm standard deviation.

\begin{tabular}{ll}
\hline Fungal pathogen & IC $_{\mathbf{5 0}}(\mathbf{n M})$ \\
\hline C. albicans & $395.2 \pm 1.1$ \\
C. parapsilosis & $454.5 \pm 1.5$ \\
C. glabrata & $550.7 \pm 2.2$ \\
C. tropicalis & $520.6 \pm 0.9$ \\
\hline
\end{tabular}

\subsubsection{HIP-HOP analysis to the mode of action of EtOH-vescalagin}

As our results identified potent antifungal activity of EtOH-vescalagin, it is essential to define the mechanism of action underlying this activity. Haploinsuffiency profiling (HIP) and homozygous profiling (HOP) are genome-wide analyses that have been used to identify the drug target and genes buffering the target, respectively (Hillenmeyer et al 200x; Lee et al 2014). We thus conducted a HIP-HOP analysis to further understand the molecular basis of antifungal activity of EtOH-vescalagin (Figure 3.3). 

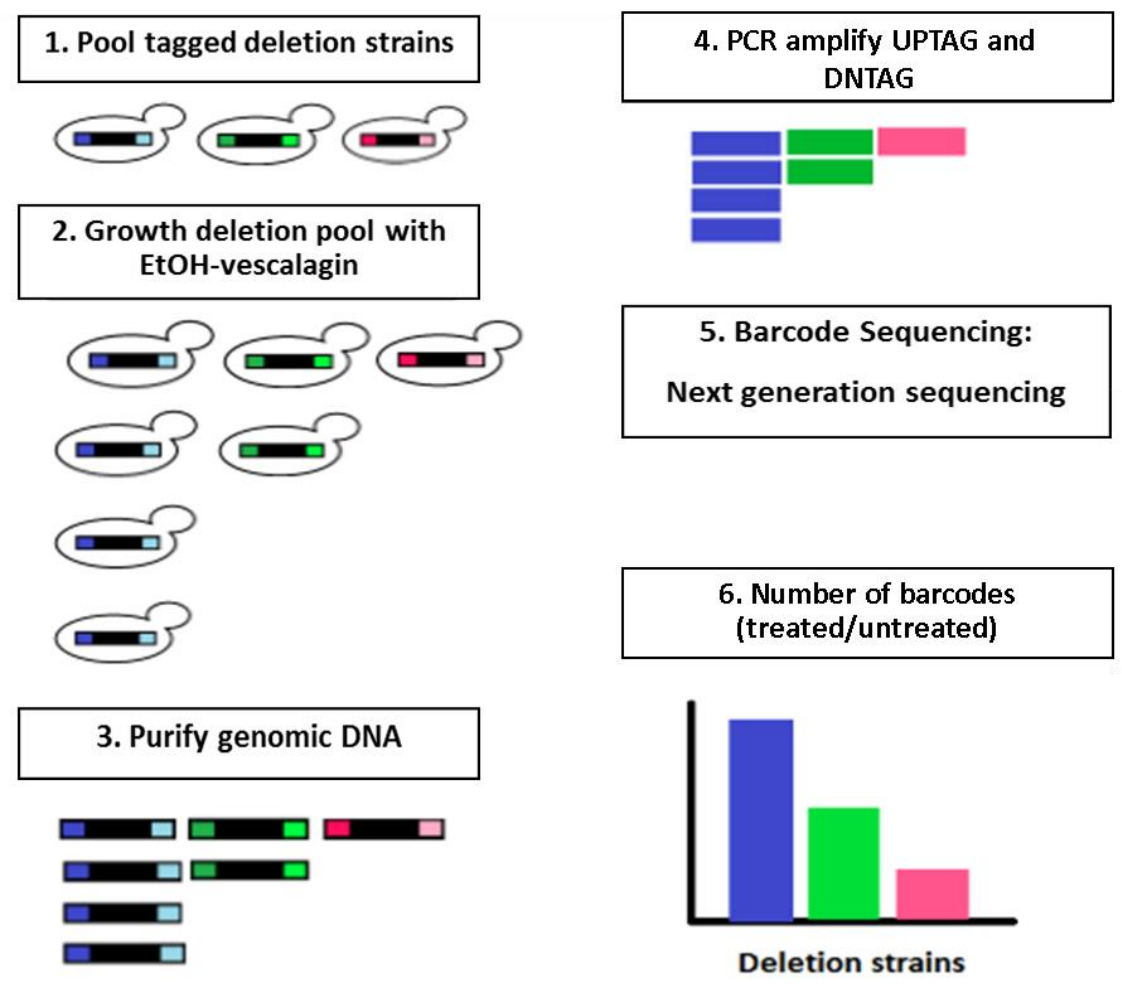

Figure 3-3. Barcode-sequencing platform. The test starts from pooling libraries and drug treatment for two sets of 10 generations, following by DNA extraction and targeted PCR and next-generation sequencing. A smaller number of barcodes shows sensitive strains and vice versa.

\subsubsection{HOP analysis identifies genes buffering the mechanism of EtOH- vescalagin}

The premise to a HOP analysis is to grow pools of the homozygous diploid mutant (HOM) library in a drug concentration that inhibits growth by only $10-20 \%$, thus leaving a large $80-90 \%$ window to detect growth reduction due to gene mutations. We thus grew the HOM library in the presence and absence of $305 \mathrm{nM} \mathrm{EtOH-vescalagin}\left(\mathrm{IC}_{20}\right)$ to mid-log and quantified the abundance of UPTAG and DNTAG barcodes flanking the 4,905 gene deletions in the HOM library. A comparison of sequencing quality ( $\log \mathrm{CPM})$ and fold change between treated and untreated cells $(\log \mathrm{FC})$ indicated an extensive distribution of fold change between -4.5 and 4 (Figure 3.4). 


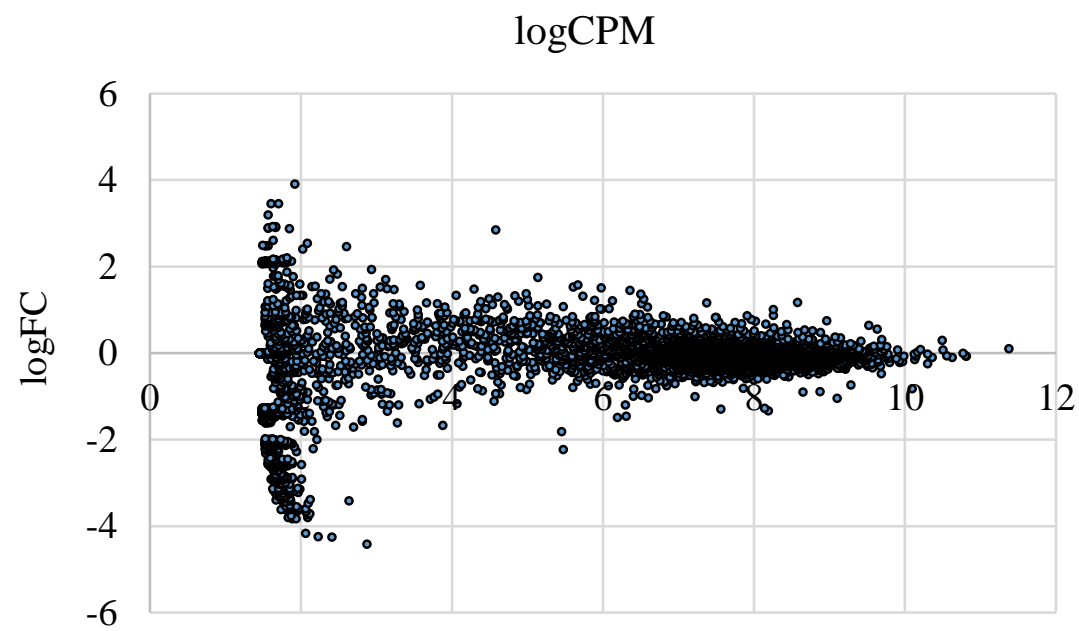

Figure 3-4. Fitness plot of HOM library in presence of EtOH-vescalagin. $\log \mathrm{CPM}$ shows the sequencing quality and $\log \mathrm{FC}$ shows the fold change in treatment compared to control. Positive logFC indicates treated sample had more growth than untreated and vice versa while $\log \mathrm{CPM}>3$ shows high quality of sequencing.

As next-generation sequencing is a high-throughput method with the potential for false positives and the competitive pooled growth assay is not a direct evaluation of specific mutations, next we sought to validate the candidate gene changes identified in the HOP analysis. We selected 151 of strains representing changes that were statistically significant (FDR $<0.05$ ), 128 of strains that exhibited $>60 \%$ inhibition independent of statistical significance, and 23 strains that exhibited $>20 \%$ growth increase. The validation assay was growth analysis of individual strains in the presence and absence of $305 \mathrm{nM}$ EtOH-vescalagin. Out of the 151 strains, 36 strains in four basic categories exhibited significant growth defects with EtOHvescalagin treatment (Figure 3.5). 


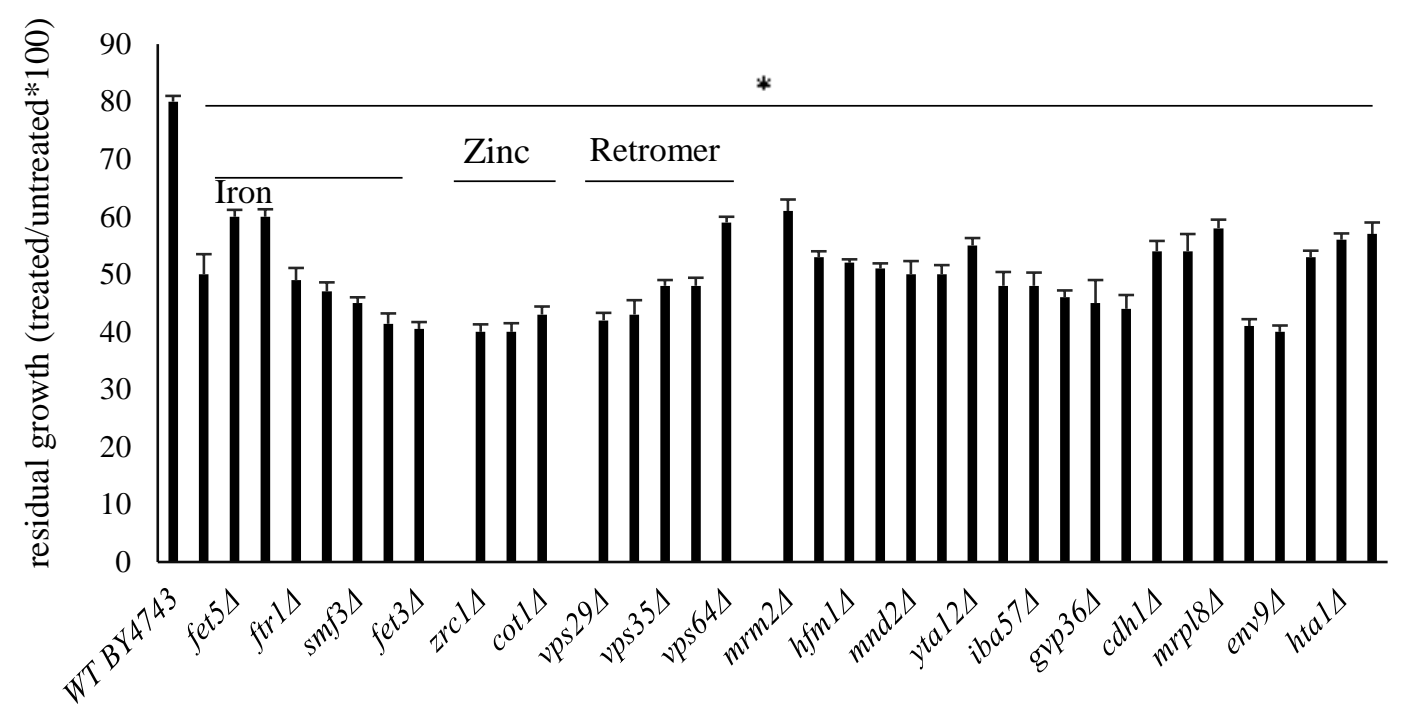

Figure 3-5. Validation of sensitive homozygous deletion strains in presence of EtOH-vescalagin. All strains were grown with and without $\mathrm{EtOH}$-vescalagin in triplicate and compared to relative control. Growth was measured after 14 hours using Envision plate reader at wavelength of $600 \mathrm{~nm}$. Residual growth of all strains were plotted as mean $\pm \mathrm{SD}$. *, $p<0.05$, Student's $t$-test comparison with WT.

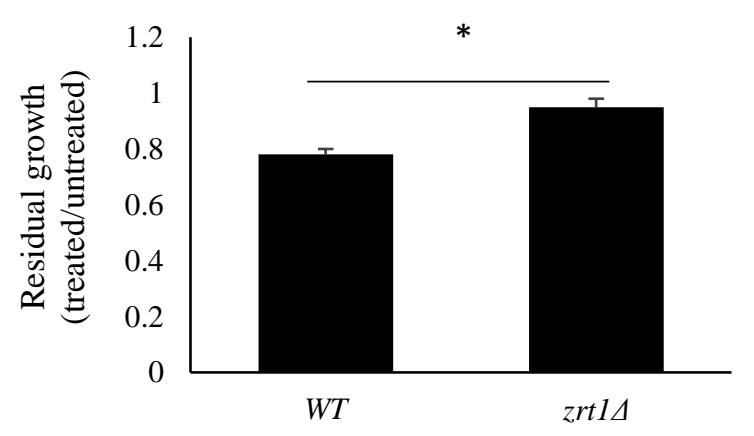

Figure 3-6. The zrt1 $\Delta$ deletion strain grew better in presence of EtOH-vescalagin. All strains were grown with and without EtOH-vescalagin in triplicate and compared to relative control. Growth was measured after 14 hours using Envision plate reader at wavelength of $600 \mathrm{~nm}$. Residual growth of all strains were plotted as mean \pm SD. $*, p<0.05$, Student's $t$-test comparison with WT. 
First, iron transport was implicated via iron transporters that transport iron into the cell (FTR1 and FET3) (Kwok, et al. 2006) or into intracellular organelles (SMF3 and FET5) (Courel, et al. 2005). Second, zinc transport was implicated via zinc transporters that transport zinc into the cell (ZRT2) and into the vacuole (ZRC1 and COT1) (MacDiarmid, et al. 2000). Interestingly, there was a zinc transporter (ZRT1) deletion that grew significantly better in the presence of EtOH-vescalagin (Figure 3.6). Third, the retromer complex was implicated via genes involved in the retromer recycling pathway (VPS17, VPS35, VPS26, VPS29, VPS64) that are responsible for retrograde endosome-to-Golgi transport (Burda, et al. 2002). Fourth, mitochondria activity was implicated via genes integral to mitochondria function (IBA57, MRPL8 and MRM2) (Lill, et al. 2012; Pintard, et al. 2002) (Table 3.3). These results thus implicate iron homeostasis, zinc homeostasis, retromer recycling and mitochondrial defects as possible mechanisms of EtOH-vescalagin bioactivity. 
Table 3-3. Homozygous gene deletions sensitive and resistant to EtOH-vescalagin and involved functions. ${ }^{*}$, resistant.

\begin{tabular}{|c|c|c|}
\hline Systematic name & Gene & Biological function \\
\hline YPL215W & CBP3 & Cytochrome B mRNA Processing \\
\hline YLR220W & $\mathrm{CCC} 1$ & Cross-Complements $\mathrm{Ca}(2+)$ phenotype of $\operatorname{csg} 1$ \\
\hline YDR270W & $\mathrm{CCC} 2$ & Cross-Complements $\mathrm{Ca}(2+)$ phenotype of $\operatorname{csg} 1$ \\
\hline YGL003C & $\mathrm{CDH} 1$ & CDC20 Homolog \\
\hline YOR316C & COT1 & CObalt Toxicity \\
\hline YPR124W & CTR1 & Copper TRansport \\
\hline YOR246C & ENV9 & Late ENdosome and Vacuole interface function \\
\hline YMR058W & FET3 & FErrous Transport \\
\hline YMR319C & FET4 & FErrous Transport \\
\hline YLR214W & FRE1 & Ferric REductase \\
\hline YKL220C & FRE2 & Ferric REductase \\
\hline YER145C & FTR1 & Fe TRansporter \\
\hline YDL100C & GET3 & Guided Entry of Tail-anchored proteins \\
\hline YIL041W & GVP36 & Golgi Vesicle Protein \\
\hline YGL251C & HFM1 & Helicase Family Member \\
\hline YDR225W & HTA1 & Histone h Two A \\
\hline YJR122W & IBA57 & $\begin{array}{l}\text { Iron-sulfur cluster assembly factor for Biotin } \\
\text { synthase }\end{array}$ \\
\hline YNL323W & LEM3 & Ligand-Effect Modulator \\
\hline YNR073C & MAN2 & MANnitol dehydrogenase \\
\hline YIR025W & MND2 & Meiotic Nuclear Divisions \\
\hline YGL136C & MRM2 & Mitochondrial rRNA Methyl transferase \\
\hline YJL063C & MRPL8 & Mitochondrial Ribosomal Protein, Large subunit \\
\hline YJL053W & PEP8 & CarboxyPEPtidase Y-deficient \\
\hline YJL136C & RPS21B & Ribosomal Protein of the Small subunit \\
\hline YLR034C & SMF3 & Metal ion transporter involved in iron homeostasis \\
\hline YKL086W & SRX1 & SulfiRedoXin \\
\hline YHR178W & STB5 & Sin Three Binding protein \\
\hline YOR132W & VPS17 & Vacuolar Protein Sorting \\
\hline YHR012W & VPS29 & Vacuolar Protein Sorting \\
\hline YJL154C & VPS35 & Vacuolar Protein Sorting \\
\hline YDR200C & VPS64 & Vacuolar Protein Sorting \\
\hline YDR269C & YDR269C & Unknown \\
\hline YGR290W & YGR290W & Unknown \\
\hline YMR089C & YTA12 & Yeast Tat-binding Analog \\
\hline YMR243C & $\mathrm{ZRC1}$ & Zinc Resistance Conferring \\
\hline YGL255W* & ZRT1 & High-affinity zinc transporter \\
\hline YLR130C & ZRT2 & Zinc-Regulated Transporter \\
\hline
\end{tabular}




\subsubsection{HIP analysis of Et-OH-vescalagin identifies sensitive HET strains}

The premise to a HIP analysis is to grow pools of the heterozygous diploid mutant (HET) library in a drug concentration that inhibits growth by only $10-20 \%$, thus leaving a large 80-90\% window to detect haploinsufficiency, a phenomenon in heterozygotes where the one gene copy is specifically inhibited by the drug resulting in dramatic growth reduction (hypersensitivity). We thus grew the HET library in the presence and absence of $305 \mathrm{nM} \mathrm{EtOH}-$ vescalagin $\left(\mathrm{IC}_{20}\right)$ to mid-log and quantified the abundance of UPTAG and DNTAG barcodes flanking the 6,153 gene deletions in the HET library. A comparison of sequencing quality $(\log \mathrm{CPM})$ and fold change between treated and untreated cells $(\operatorname{logFC})$ indicated an extensive distribution of fold change between -4.1 and 2.7 (Figure 3.7).

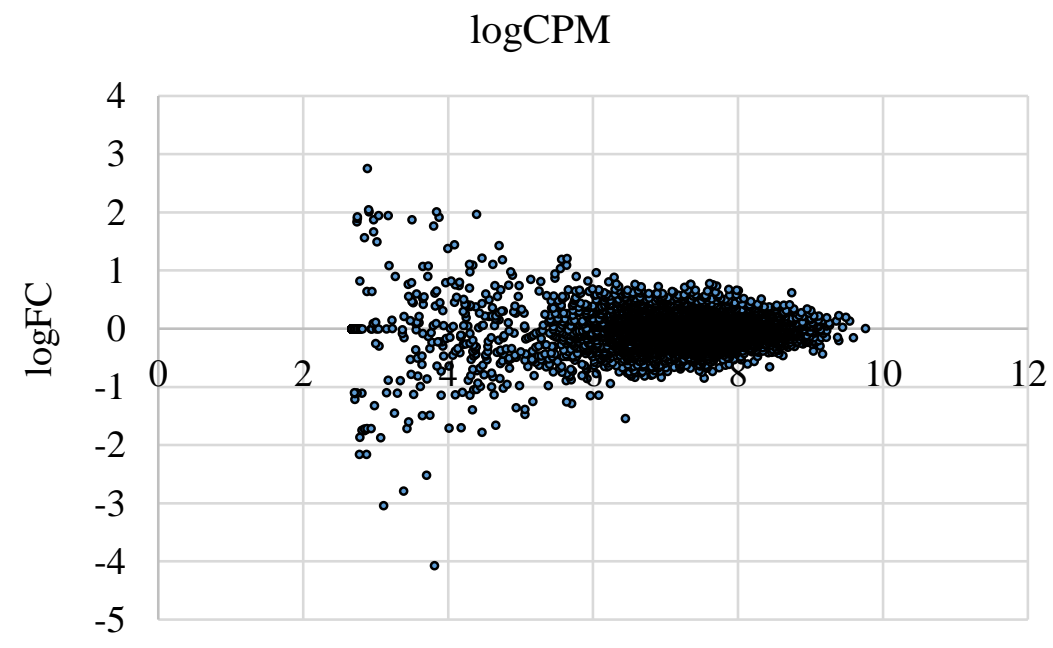

Figure 3-7. Fitness plot of HET library in presence of EtOH-vescalagin. Read counts were compared in treatment with control. Higher $\operatorname{logFC}$ indicates more changes in read counts while negative values mean mutants grew less and positive values show mutants grew better. $\log C P M>3$ shows high quality of sequencing (greater than 2 ). 
As next-generation sequencing is a high-throughput method with the potential for false positives and the competitive pooled growth assay is not a direct evaluation of specific mutations, next we sought to validate the candidate gene changes identified in the HIP analysis. The HIP analysis did not result in any statistically significant changes, thus the only strains selected for validation were 97 strains that exhibited $>60 \%$ inhibition independent of statistical significance. The validation assay was growth analysis of individual strains in the presence and absence of $305 \mathrm{nM}$ EtOH-vescalagin. Out of the 97 strains, 16 strains in 5 basic categories exhibited significant growth defects with EtOH-vescalagin treatment (Figure 3.7; Table 3.4). The first group was nuclear function (NRD1, UTP22, ADE5, DBF4), the second group was mitochondrial function (RRG8, FMP33), the third group was protein biosynthesis (the RNA polymerase RPC53, the DNA polymerase POL31, the ribosomal protein KAP123), and the fourth group was a random assortment of pathways such as core histone protein (HTA1), cell division function (CDC19), bud site selection (BUD19), adenylate cyclase (CYR1), phosphatidylserine decarboxylase (PSD1), and J-type Accessory Chaperone (JAC1). Together, these 16 genes are possibly mediating the primary target of EtOH-vescalagin (Figure 3.8). 


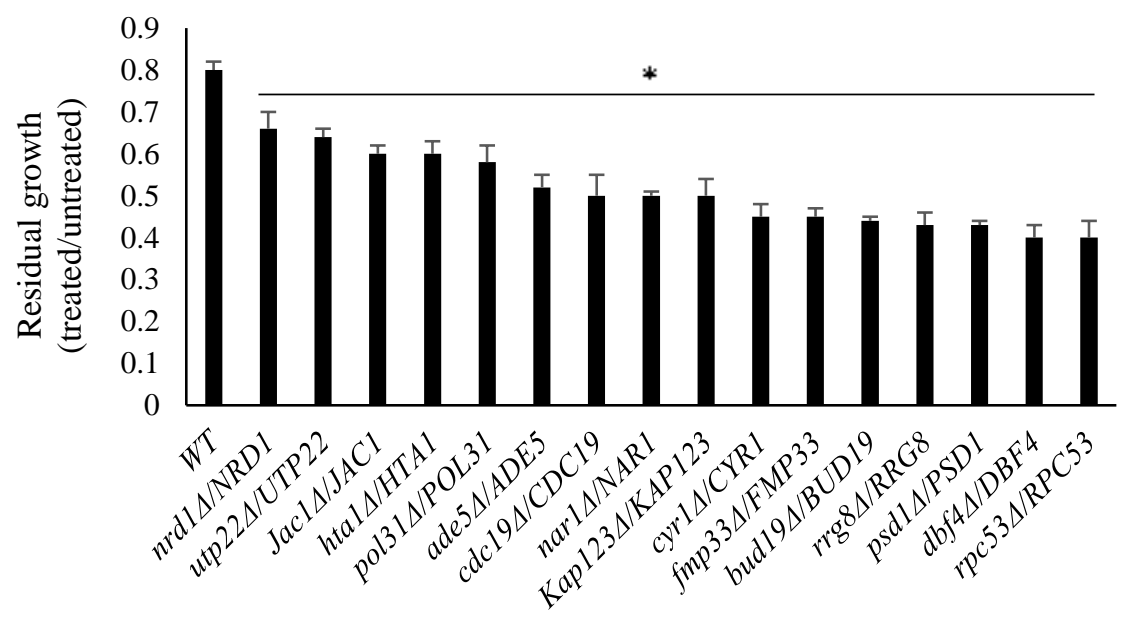

Figure 3-8. Validation of 16 sensitive heterozygous deletion strains in presence of EtOHvescalagin. All strains were grown with and without EtOH-vescalagin in triplicate and compared to relative control. Growth was measured after 14 hours using Envision plate reader at wavelength of 600 $\mathrm{nM}$. Residual growth of all strains were plotted as mean $\pm \mathrm{SD}$. *, $p<0.05$, Student's $t$-test comparison with WT.

Table 3-4. Heterozygous gene deletions sensitive to EtOH-vescalagin.

\begin{tabular}{cll}
\hline Systematic name & Gene & Biological function \\
\hline YNL251C & NRD1 & Nuclear pre-mRNA Down-regulation \\
YGR090W & UTP22 & Required for nuclear export of tRNAs \\
YGL018C & JAC1 & J-type Accessory Chaperone \\
YDR225W & HTA1 & Core histone protein \\
YJR006W & POL31 & Subunit of DNA polymerase delta (polymerase III \\
YGL234W & ADE5 & Purine nucleotide biosynthetic pathway \\
YAL038W & CDC19 & Cell Division Cycle \\
YNL240C & NAR1 & Nuclear Architecture Related \\
YER110C & KAP123 & Mediates nuclear import of ribosomal protein \\
YJL005W & CYR1 & CYclic AMP Requirement \\
YJL161W & FMP33 & Mitochondrial Proteome \\
YJL188C & BUD19 & BUD site selection \\
YPR116W & RRG8 & Required for Respiratory Growth \\
YNL169C & PSD1 & PhosphatidylSerine Decarboxylase \\
YDR052C & DBF4 & Required for Cdc7p kinase activity and initiation of DNA replication \\
YDL150W & RPC53 & RNA Polymerase C \\
\hline
\end{tabular}




\subsubsection{Gene Ontology analyses distinguish metal homeostasis and the retromer complex}

To gain more insight into results of the HIP-HOP analysis, we evaluated the functions, processes and components of the genes sensitive to EtOH-vescalagin for enrichment relative to the whole genome. All genes from the HIP and HOP analyses were pooled and tested for enrichment relative to the whole genome using YeastMap (Balakrishnan, et al. 2012). For biological process, there was significant enrichment in metal ion transition, cellular iron homeostasis and zinc ion transport (Table 3.5). For biological function, there was significant enrichment in metal ion transmembrane transporter activity (Table 3.5). For cellular component, there was significant enrichment in the retromer complex (Table 3.5). These results suggest that EtOH-vescalagin is impacting iron transport, zinc transport, and the retromer complex.

Table 3-5. Gene Ontology analysis of sensitive HET and HOM deletion strains to EtOHvescalagin. Analysis was conducted using YeastMine and a Holm-Bonferroni correction.

\begin{tabular}{llccc}
\hline GO term & Function & $\begin{array}{l}\text { No. Genes in } \\
\text { HIP-HOP }\end{array}$ & $\begin{array}{l}\text { No. Genes in } \\
\text { GO category }\end{array}$ & $\boldsymbol{p}$-value \\
\hline biological process & $\begin{array}{l}\text { transition metal ion } \\
\text { transport }\end{array}$ & 12 & 75 & $6.18 \mathrm{E}-12$ \\
\hline biological process & $\begin{array}{l}\text { cellular iron ion } \\
\text { homeostasis }\end{array}$ & 7 & 19 & $4.13 \mathrm{E}-04$ \\
\hline biological process & zinc II ion transport & 4 & 12 & 0.009129 \\
\hline cellular component & retromer complex & 4 & 6 & $1.39 \mathrm{E}-05$ \\
\hline molecular function & $\begin{array}{l}\text { Metal ion transmembrane } \\
\text { transporter activity }\end{array}$ & 8 & 58 & $2.19 \mathrm{E}-07$ \\
\hline
\end{tabular}




\subsubsection{Iron, zinc and copper supplementation impacts EtOH-vescalagin bioactivity}

Iron is the most abundant metal in eukaryotic cells (Bleackley and MacGillivray 2011). The uptake of iron is regulated by a few genes encoding proteins in the plasma membrane, such as the genes regulating high affinity iron transport (FET3, FTR1), low affinity transport (FET4), and also the seven genes that reduce Fe (III) to Fe (II) to allow for uptake (FRE1-7) (Georgatsou and Alexandraki 1994). Once inside the cell, iron homeostasis is largely regulated by iron transporters at the vacuole (FET5, FTH1, SMF3) that transport iron out of the vacuole in response to iron limitation (Figure 3.9) (Singh, et al. 2007).

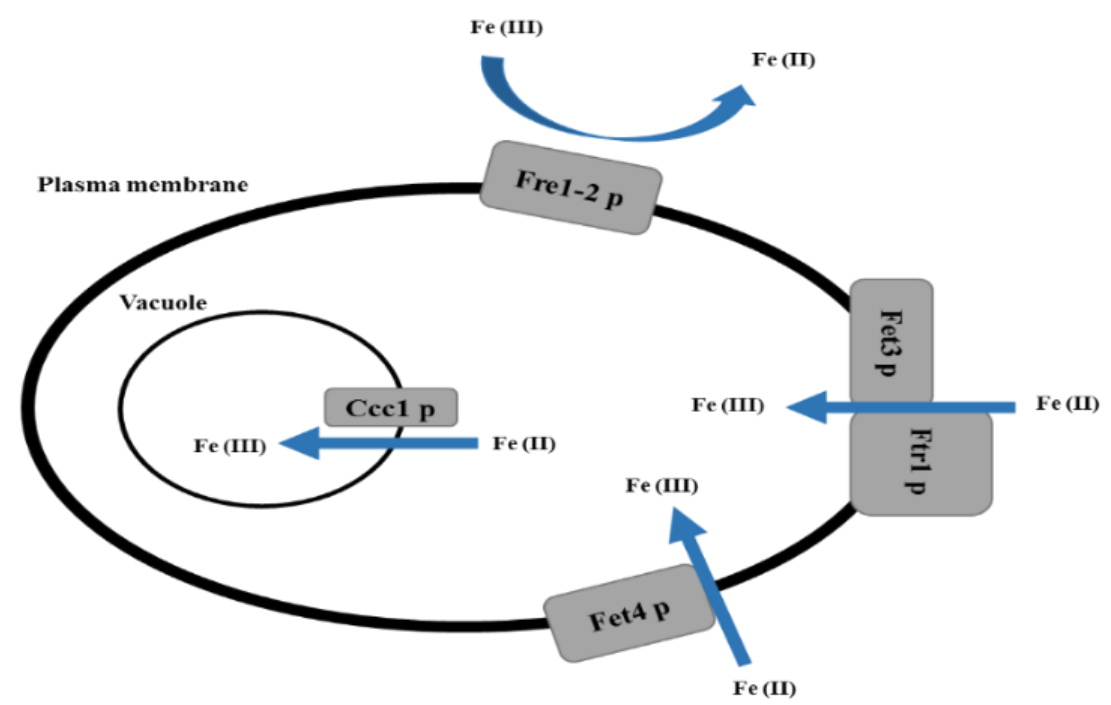

Figure 3-9. Iron homeostasis in yeast.

Given that the HOP analysis identified iron transporter deletion strains (fet $3 \Delta$, ftr $1 \Delta$, fre2 $\Delta$, fet5 $4, s m f 3 \Delta$, fre 14 ) sensitive to $\mathrm{EtOH}$-vescalagin, we hypothesized EtOH-vescalagin treatment results in reduced intracellular iron levels, and if so, iron supplementation would rescue the inhibition. We thus quantified growth of WT cells in the presence of varying 
concentrations of $\mathrm{FeCl}_{3}$ and $\mathrm{EtOH}$-vescalagin (Figure 3.10). As expected, increasing concentrations of EtOH-vescalagin resulted in increased toxicity, such as the 41, 72 and $100 \%$ inhibition with treatments of 500,900 and $1100 \mathrm{nM}$, respectively. The supplementation of $25 \mu \mathrm{M} \mathrm{FeCl}_{3}$ partially rescued these inhibition values by $15-20 \%$, while supplementation with either $50 \mu \mathrm{M} \mathrm{FeCl}_{3}$ or $100 \mu \mathrm{M} \mathrm{FeCl}{ }_{3}$ completely rescued these inhibition values. These results suggest that EtOH-vescalagin reduces intracellular levels of iron (Figure 3.10).

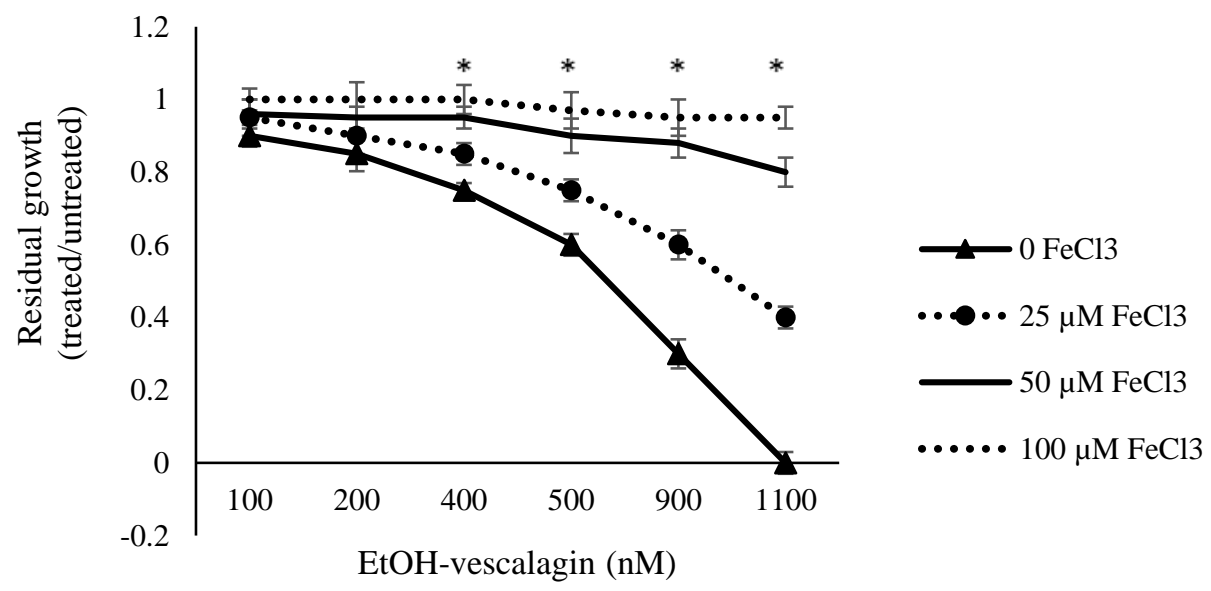

Figure 3-10. Iron supplementation in the presence of EtOH-vescalagin. Increasing concentrations of EtOH-vescalagin and $\mathrm{FeCl}_{3}$ were used in WT BY4741 yeast strain. *, $p<0.05$ Student's $t$-test comparison of zero $\mathrm{FeCl}_{3}$ and other concentrations.

Zinc is the second most abundant metal in eukaryotic cells (Tapiero and Tew 2003). In $S$. cerevisiae, the uptake of zinc is regulated by a few genes encoding proteins in the plasma membrane such as the high affinity zinc transporter (ZRT1) that is upregulated in zinc-limiting conditions and the low affinity transporter (ZRT2) that is upregulated in zinc-replete conditions (Zhao and Eide 1997). Once inside the cell, zinc homeostasis is largely regulated by zinc transporters at the vacuole (ZRC1, COT1) and the endoplasmic reticulum (MSC2, ZRG17) (Simm, et al. 2007) (Figure 3.11). 


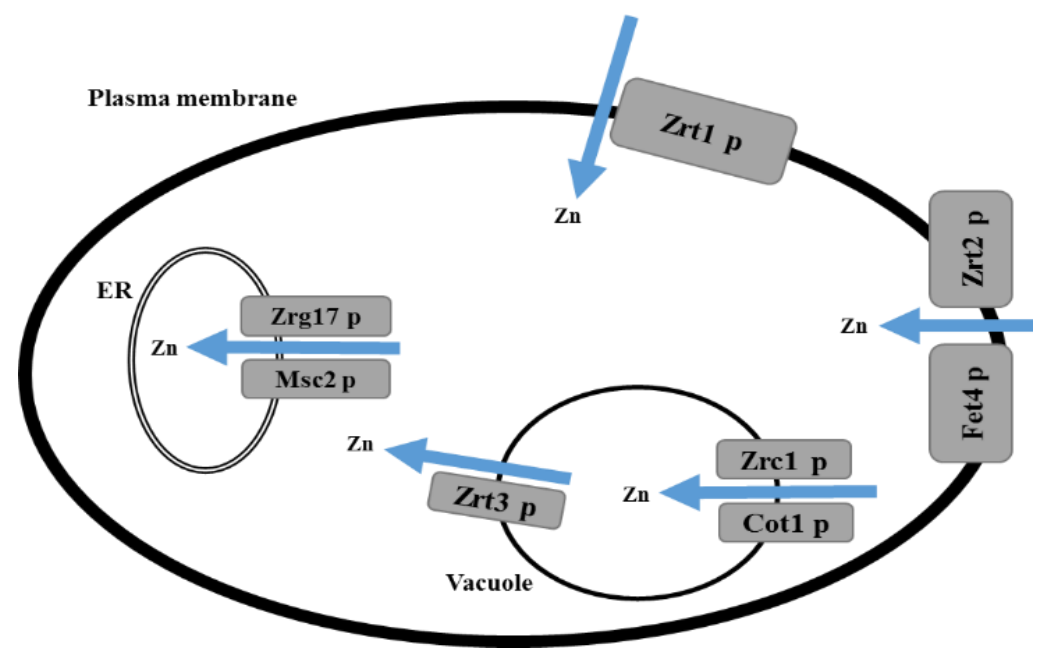

Figure 3-11. Zinc homeostasis in yeast.

Given that zinc metabolism is tightly regulated with iron metabolism (Waters and Eide 2002) and that the HOP analysis identified zinc transporter deletion strains (zrc14, cot1A. $m s c 2 \Delta, \operatorname{zrg} 174)$ sensitive to EtOH-vescalagin, we hypothesized EtOH-vescalagin treatment results in reduced intracellular zinc levels, and if so, zinc supplementation would rescue the inhibition. We thus quantified growth of WT cells in the presence of varying concentrations of $\mathrm{ZnCl}_{2}$ and EtOH-vescalagin (Figure 3.12). As expected, increasing concentrations of EtOH-vescalagin resulted in increased toxicity, such as the 41, 72 and $100 \%$ inhibition with treatments of 500,900 and $1100 \mathrm{nM}$, respectively. The supplementation of $12 \mu \mathrm{M} \mathrm{ZnCl}_{2}$ did not significantly affect growth inhibition at any EtOHvescalagin concentration. In contrast to iron supplementation, the addition of $25 \mu \mathrm{M} \mathrm{ZnCl}_{2}$ or $50 \mu \mathrm{M} \mathrm{ZnCl} 2$ significantly increased toxicity at mid-range concentrations of EtOHvescalagin $(400,500,900 \mathrm{nM})$, albeit these increases were only between $10-15 \%$. These results suggest that EtOH-vescalagin increases intracellular levels of zinc. 


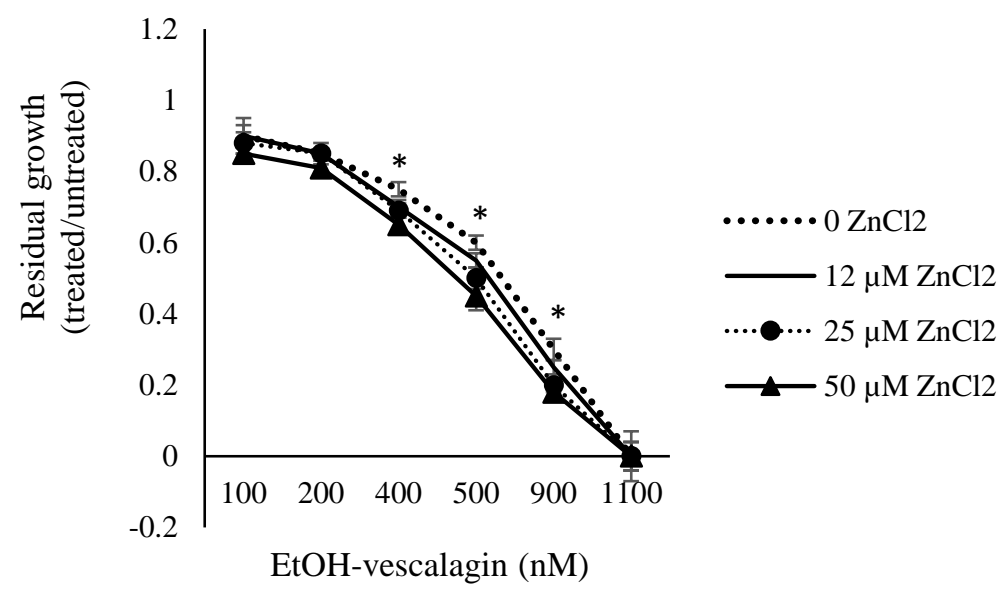

Figure 3-12. Zinc supplementation in the presence of EtOH-vescalagin. Increasing concentrations of EtOH-vescalagin were mixed with increasing concentrations of $\mathrm{ZnCl}_{2}$ in WT BY4741. *, $p<0.05$, Student's $t$-test comparison of zero $\mathrm{ZnCl}_{2}$ and other concentrations.

Copper is the third abundant metal in eukaryotic cells (Cyert and Philpott 2013). The uptake of copper is regulated by a few genes encoding proteins in the plasma membrane, such as the genes regulating high affinity copper transport (CTR1, CTR3) (Rees, et al. 2004), while a transporter at the vacuole (CTR2) regulates storage of excess copper in the vacuole (Figure 3.13).

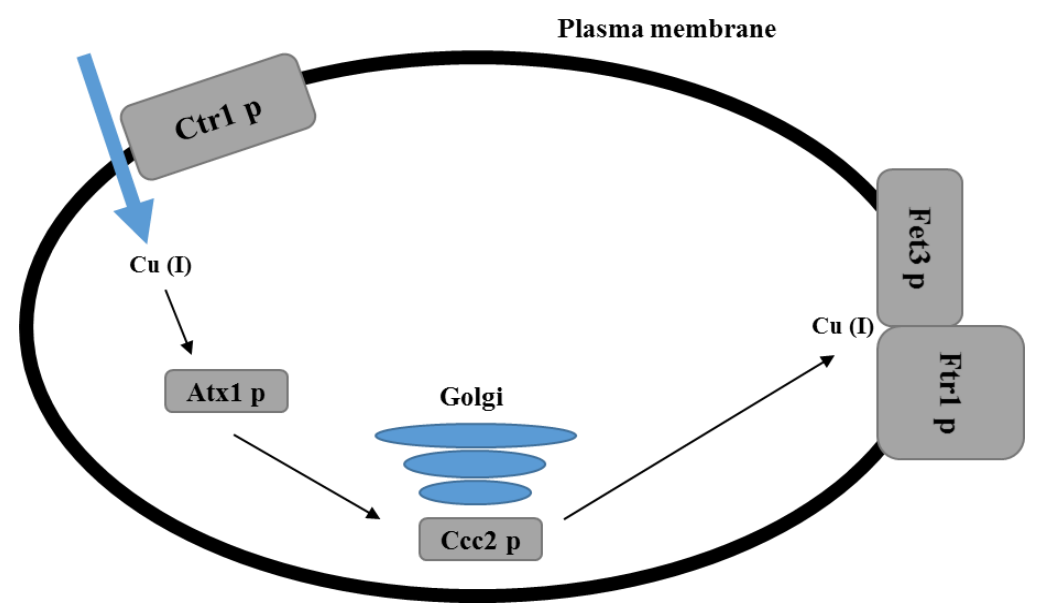

Figure 3-13. Copper homeostasis in yeast. 
Given that copper metabolism is tightly regulated with iron metabolism (Rustici, et al. 2007) and that the HOP analysis identified a copper transporter deletion strain (ctr14) sensitive to EtOH-vescalagin, we hypothesized EtOH-vescalagin treatment results in reduced intracellular copper levels, and if so, copper supplementation would rescue the inhibition. We thus quantified growth of WT cells in the presence of varying concentrations of $\mathrm{FeCl}_{3}$ and EtOH-vescalagin (Figure 3.14). As expected, increasing concentrations of EtOH-vescalagin resulted in increased toxicity, such as the 41, 72 and $100 \%$ inhibition with treatments of 500, 900 and $1100 \mathrm{nM}$, respectively. The supplementation of $\mathrm{CuCl}_{3}$ levels as low as $1.5 \mu \mathrm{M}$ partially rescued the growth inhibition caused by 300,500 and $700 \mathrm{nM}$ EtOHvescalagin, but had no effect on the $>50 \%$ inhibition caused by 900 and $1100 \mathrm{nM} \mathrm{EtOH}-$ vescalagin. These results suggest that EtOH-vescalagin reduces intracellular levels of copper but not as dramatically as that of iron.

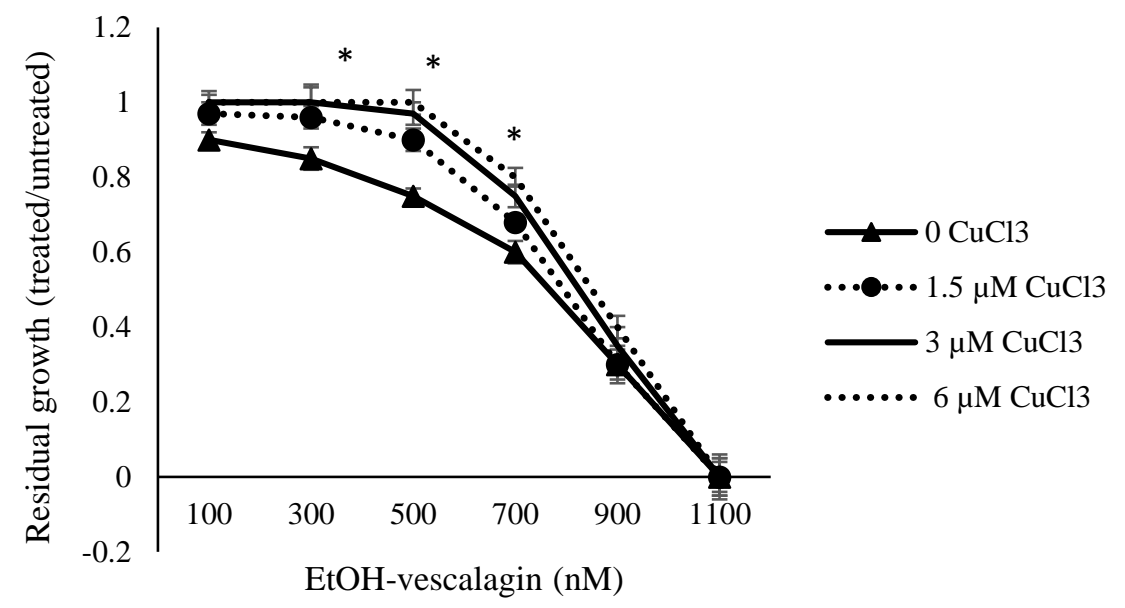

Figure 3-14. Copper supplementation in presence of EtOH-vescalagin. Increasing concentration of EtOH-vescalagin was mixed with increasing concentrations of $\mathrm{CuCl}_{2}$ in WT BY4741. Triangle marker line in control without any copper added. *, Student's $t$-test comparison of Zero $\mathrm{CuCl}_{2}$ and different concentrations, $p$-value $<0.05$. 


\subsubsection{Zinc and iron mediate retromer-specific sensitivity to EtOH-}

vescalagin

Endocytosed cargo can be transported to the lysosome for degradation or recycled (via retrograde transport) back to the plasma membrane (Klinger, et al. 2015). The retromer complex, composed of two sub-complexes (the VPS26/VPS29/VPS35 complex that recognizes and sorts cargo and the VPS5/VPS10/VPS17 complex that regulates membrane formation around the cargo, is fundamental to retrograde transport from endosomes to Golgi. Given that the HOP analysis identified retromer deletion strains (vps174, vps294, vps354) sensitive to EtOH-vescalagin (Figure 3.5) and that sensitivity is influenced by levels of iron (Figure 3-10) and zinc (Figure 3-12), we hypothesized that iron and zinc supplementation would modulate the sensitivity of retromer deletion strains to $\mathrm{EtOH}$-vescalagin. We thus quantified EtOHvescalagin sensitivity of WT, vps54, vps104, vps174, vps264, vps294, and vps354 in response to varying levels of iron and zinc (Figure 3.15).

Consistent with iron supplementation completely rescuing growth inhibition in WT, $100 \mu \mathrm{M} \mathrm{FeCl}_{3}$ (optimal iron concentration) completely rescued inhibition in all six retromer deletion strains, albeit this was in the background of this iron concentration that improved growth of all strains in the absence of EtOH-vescalagin (Figure 3.15). There were basically two degrees of rescue and these were dependent on the sub-complex. The defect in vps54, vps 104 and vps 174 was restored from 52-58\% inhibition to $0-5 \%$ inhibition (normal, unaffected growth). The defect in vps264, vps294 and vps354 was restored from 47-57\% inhibition to levels twice that of normal, unaffected growth. These results indicate that iron supplementation improves growth in WT as well as the retromer deletion mutants.

Consistent with zinc supplementation exacerbating the growth defect in WT (Figure 3.16), $25 \mu \mathrm{M} \mathrm{ZnCl}_{2}$ (optimal zinc concentration) exacerbated the growth defect in WT by $21 \%$. Unlike the results with iron, the results for zinc supplementation were not completely aligned 
by the sub-complex status. For one sub-complex, the zinc supplementation did not impact EtOH-vescalagin growth defect in vps5 , while it did exacerbate the growth defect in vps 104 and $v p s 174$ by $70-78 \%$. For the other sub-complex, zinc supplementation exacerbated the growth defect in vps264, vps294 and vps354 to a lesser extent by 39-54\%. Intriguingly, the retromer deletions were variably sensitive to zinc supplementation independent of $\mathrm{EtOH}-$ vescalagin treatment. Deletion strains in the VPS26/VPS29/VPS35 as well as WT were not sensitive to zinc, while deletion strains in the VPS5/VPS10/VPS17 complex were sensitive to zinc supplementation (i.e., growth of vps $5 \Delta$ was increased by $29 \%$ while growth of $v p s 10 \Delta$ and vps 174 was reduced by 22-25\%). These results indicate that EtOH-vescalagin sensitivity of retromer deletion strains is exacerbated with zinc and that the VPS5/VPS10/VPS17 complex is sensitive to zinc independent of EtOH-vescalagin. Moreover, the iron and zinc supplementation results indicate that the mechanism of action of EtOH-vescalagin includes altered iron homeostasis, zinc homeostasis, and retromer recycling.

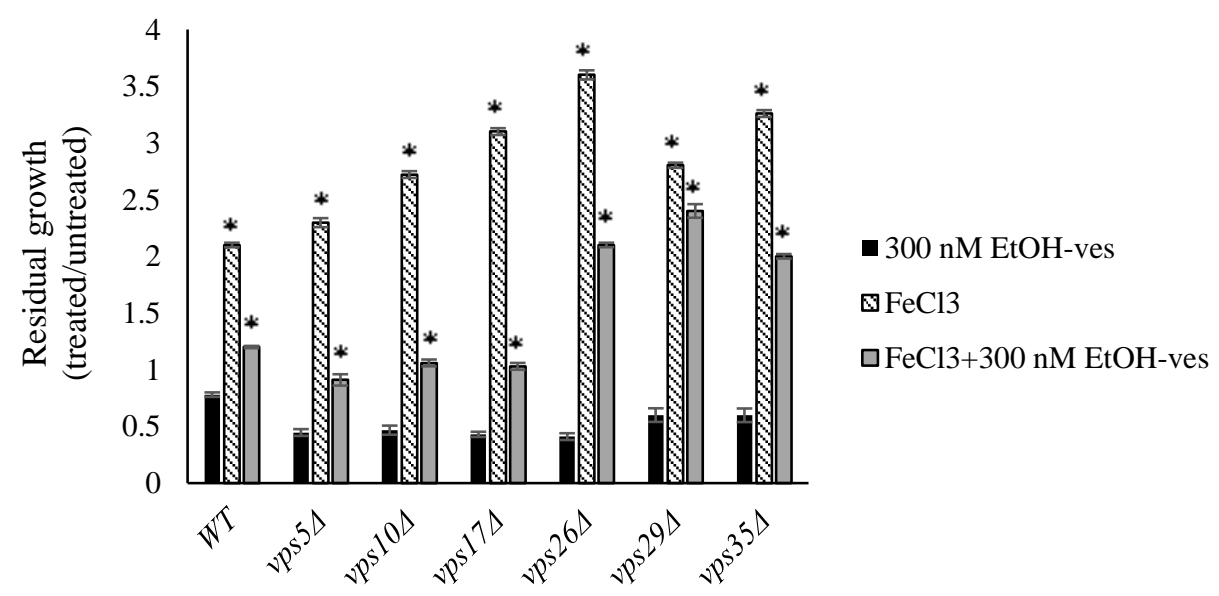

Figure 3-15. Retromer deletion strains respond to iron. All data values are in triplicate mean \pm $\mathrm{SD}$ compared to relative control. The growth was measured after 14 hours at $\mathrm{OD}_{600}$ by Envision plate reader (Perkin Elmer). ${ }^{*}, p<0.05$, Student's t-test comparison with EtOH-vescalagin (EtOHves). 


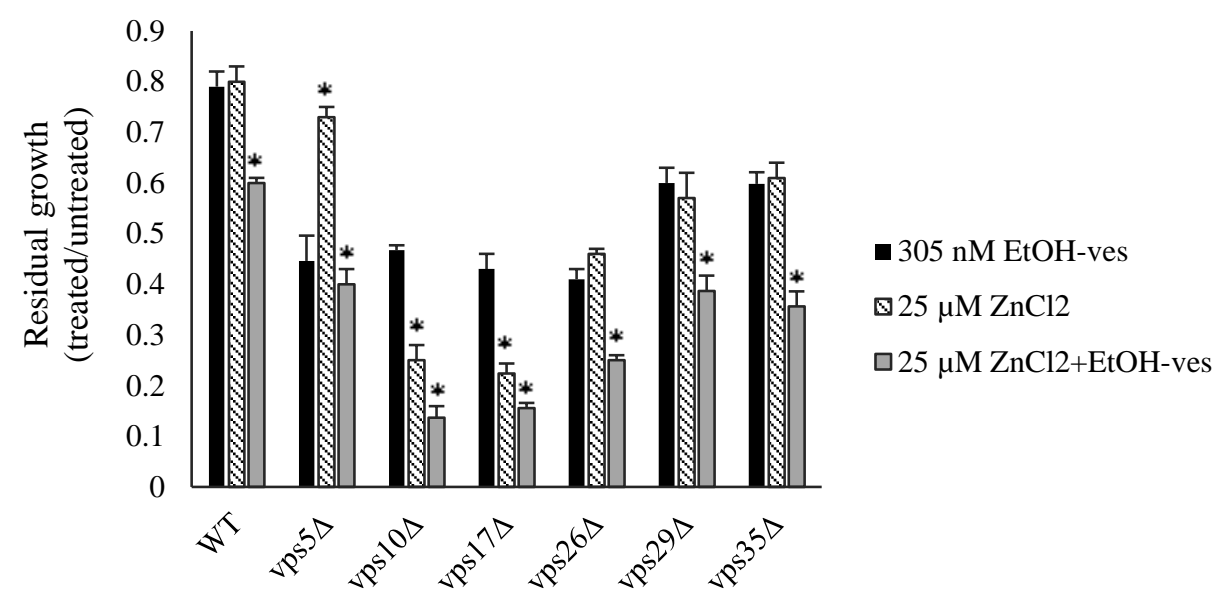

Figure 3-16. Retromer deletion strains respond to zinc. All data values are in triplicate mean \pm $\mathrm{SD}$ compared to relative control. The growth was measured after 14 hours at $\mathrm{OD}_{600}$ by Envision plate reader (Perkin Elmer). *, $p<0.05$, Student's $t$-test comparison with EtOH-vescalagin.

\subsubsection{Vacuolar storage of zinc buffers EtOH-vescalagin toxicityn}

To further understand our observation that zinc supplementation exacerbates EtOHvescalagin sensitivity (Figure 3.12), we investigated zinc transport in different cellular compartments. Zinc uptake occurs at the plasma membrane via the high affinity transporter ZRT1 and the low affinity transporter ZRT2, and then intracellular zinc is transported into the vacuole by ZRC1 and COT1 or into the endoplasmic reticulum by MSC2 and ZRG17 (Eide 2006). We thus evaluated the contributions of these genes via deletion strains (gift of David Eide, University of Wisconsin). Relative to WT, zrt1A was significantly less sensitive to EtOHvescalagin (10\% growth increase) while zrt2ム was significantly more sensitive to $\mathrm{EtOH}$ vescalagin (63\% growth decrease), a result consistent with a model that EtOH-vescalagin increases intracellular zinc via ZRT1 (Figure 3.17). As the expression of ZRT1 and ZRT2 are dependent on intracellular zinc levels (e.g., ZRT2 requires increased zinc for increased expression, and vise-versa for ZRT1) (Zhao, et al. 1998), the different sensitivity of zrt14 and 
zrt2 $\Delta$ is likely a consequence of the zinc dosage in the cell upon EtOH-vescalagin treatment. The single deletions regulating vacuolar zinc transport $(\operatorname{zrc1\Delta }, \cot 1 \Delta)$ were each sensitive to EtOH-vescalagin with $\sim 45 \%$ growth defect, while the $\operatorname{zrc1} 1 \Delta \cot 1 \Delta$ double deletion exhibited greater sensitivity with a $76 \%$ growth defect. The single deletions regulating ER zinc transport ( $m s c 24, \operatorname{zrg} 174)$ were each sensitive to EtOH-vescalagin with $\sim 40 \%$ growth defect, while the msc2 4 rg 174 double deletion did not exhibit any significant difference in EtOH-vescalagin sensitivity compared to the single deletions (Figure 3.17). In addition, the zrc1 $1 \Delta \cot 1 \Delta m s c 2 \Delta z \operatorname{rg} 17 \Delta$ quadruple deletion strain was not significantly different from the zrcl $1 \Delta \cot 1 \Delta$ double deletion. Since the $z r c 1 \Delta \cot 1 \Delta$ and $m s c 2 \Delta \operatorname{zrg} 17 \Delta$ strains exhibit an intracellular accumulation of zinc (Eide 2006), the EtOH-vescalagin sensitivity in all single deletion strains suggest that intracellular zinc toxicity may be a mechanism of EtOH-vescalagin. This toxicity is a consequence of zinc uptake at the plasma membrane as well as intracellular

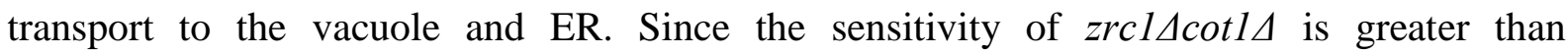
$m s c 2 \Delta z \operatorname{rg} 17 \Delta$ and not different from $\operatorname{zrc} 1 \Delta \cot 1 \Delta m s c 2 \Delta z \operatorname{rg} 17 \Delta$, it is likely that vacuolar storage is a more critical buffering mechanism than ER zinc level.

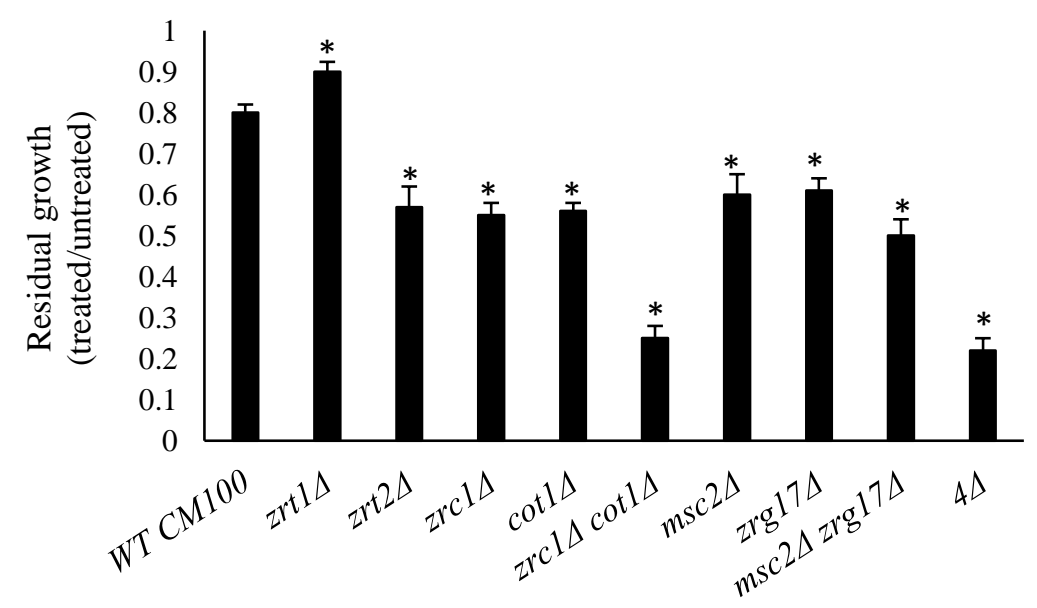

Figure 3-17. Sensitivity of EtOH-vescalagin in zinc transporters deletion strains in vacuole and ER. All absorbance measure at $\mathrm{OD}_{600}$ after 14 hours. *, $p<0.05$, Student's $t$-test comparison with WT. 4D is quadruple mutant. 


\subsubsection{EtOH-vescalagin treatment phenocopies zinc supplementation and is not a zinc chelator}

The results thus far suggest that EtOH-vescalagin increases intracellular levels of zinc. To test whether this increase is due to zinc chelation, growth of select strains (WT, zrc1 1 cot1 1 , zrc1 1 cot $1 \Delta m s c 2 \Delta z r g 174)$ was compared for sensitivity to EtOH-vescalagin and the zinc chelator TPEN (Figure 3.18). Both treatments inhibited growth of WT by 20\%, yet had dramatically different results in $\operatorname{zrc} 1 \Delta \cot 1 \Delta$ and $z r c 1 \Delta \cot 1 \Delta m s c 2 \Delta z r g 17 \Delta$. EtOH-vescalagin reduced growth of $z r c 1 \Delta \cot 1 \Delta$ and $z r c 1 \Delta \cot 1 \Delta m s c 2 \Delta \operatorname{zrg} 17 \Delta$ by 72 and $80 \%$, respectively, compared to untreated cells. In contrast, TPEN completely restored growth of these strains to normal, unaffected levels. These results confirm that EtOH-vescalagin is not a zinc chelator. To estimate the concentration of zinc accumulation induced by EtOH-vescalagin, the three strains were grown in media containing three concentrations of zinc $\left(25,50\right.$ and $\left.100 \mu \mathrm{M} \mathrm{ZnCl}_{2}\right)$ (Figure 3.18). The treatment of $25 \mu \mathrm{M} \mathrm{ZnCl}_{2}$ in WT was not significantly different from the $305 \mathrm{nM}$ EtOH-vescalagin treatment in WT $(p=0.09)$. The treatment of $25 \mu \mathrm{M} \mathrm{ZnCl}_{2}$ in zrcl $1 \Delta \cot 1 \Delta$ was not significantly different from the $305 \mathrm{nM}$ EtOH-vescalagin treatment in

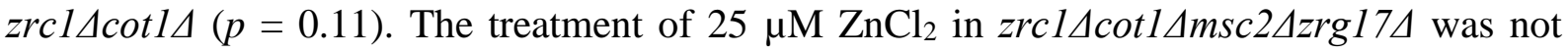
significantly different from the $305 \mathrm{nM}$ EtOH-vescalagin treatment in $z r c 1 \Delta \cot 1 \Delta m s c 2 \Delta z \operatorname{rg} 17 \Delta$ $(p=0.1)$. These results suggest that an $\mathrm{IC}_{20}$ concentration of EtOH-vescalagin induces an intraceullar zinc concentration to the same extent as a $25 \mu \mathrm{M} \mathrm{ZnCl}_{2}$ treatment. 


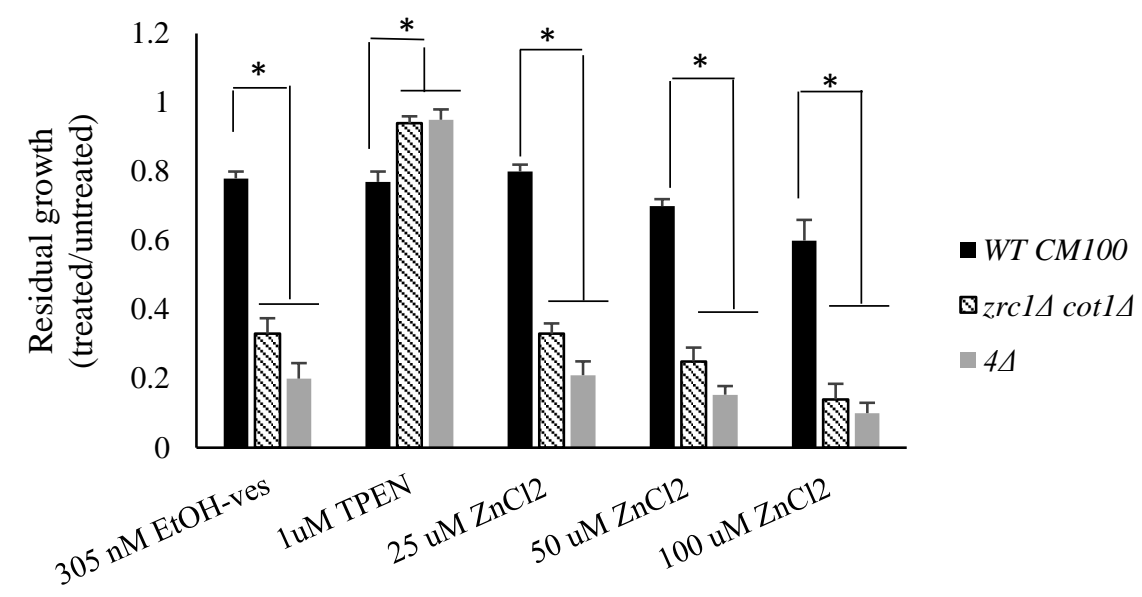

Figure 3-18. EtOH-vescalagin sensitivity of zinc transporters deletion strains to zinc chelator (TPEN) and zinc supplementation. The growth was measured after 14 hours by absorbance reading at $\mathrm{OD}_{600}$ and residual was calculated compared to relative control. ${ }^{*}, p<0.05$, Student's $t$-test comparison with WT.

\subsubsection{EtOH-vescalagin increases intracellular zincosomes}

To further understand the cellular basis by which EtOH-vescalagin increases intracellular zinc, we used the fluorescent stain Zinquin to visualize cytoplasmic zinccontaining vesicles (zincosomes) (Figure 3.19). In WT cells, EtOH-vescalagin significantly increased zincosome abundance by $25 \%$ compared to untreated cells. Likewise, in the zrt1A strain that has previously been recognized with reduced numbers of zincosomes (Yuan 2011), EtOH-vescalagin significantly increased zincosome abundance by $35 \%$ compared to untreated cells. These results indicate that the intracellular zinc toxicity caused with EtOH-vescalagin treatment is a consequence, at least partially, of an increase in zincosomes. 
A

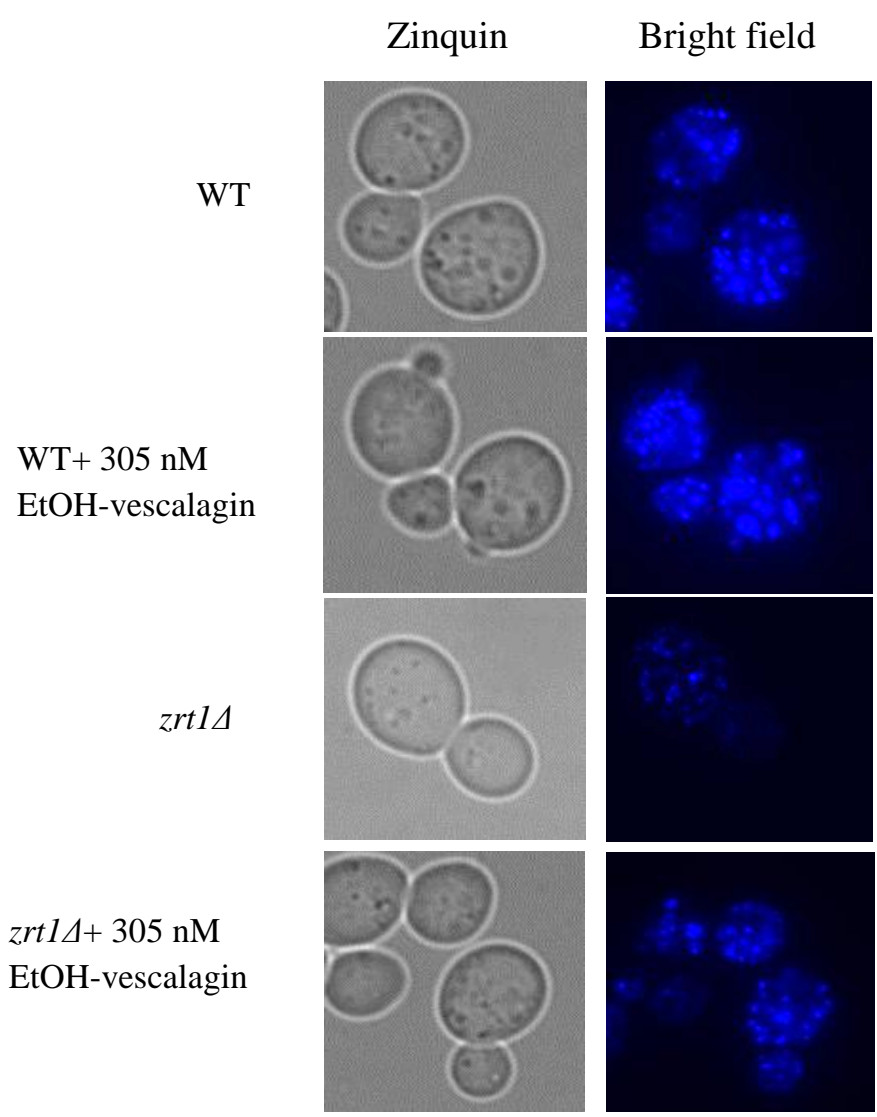

B

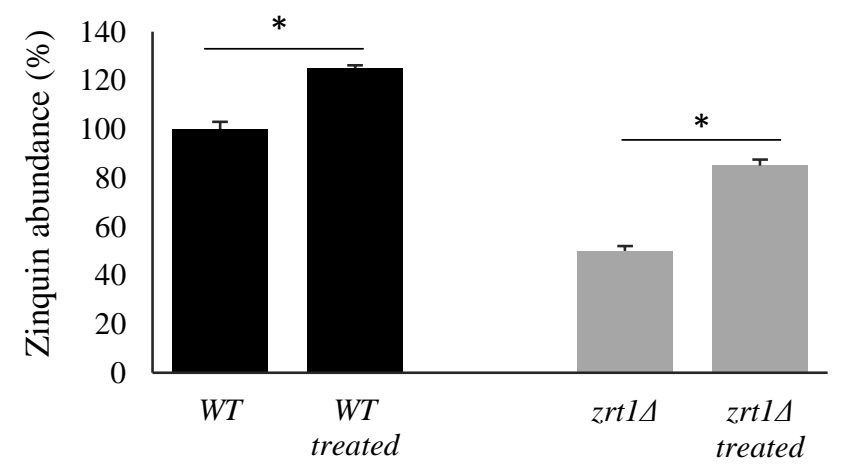

Figure 3-19. EtOH-vescalagin increases intercellular zincosomes in WT and zrt1A strains. A) Zinquin staining. Cells were treated with $305 \mathrm{nM}$ ETOH-vescalagin and grown to mid-log phase of growth. Cells were stained by zinquin for $45 \mathrm{~min}$ and visualised by fluorescent Olympus microscope using DAPI filter. B) Quantification of zinquin abundance compared to WT control was analysed by Fiji-image j. ${ }^{*}, p<0.05$, Student's $t$ test comparison with relative control. 


\subsubsection{EtOH-vescalagin increases intracellular zinc levels and decreases intracellular iron levels}

To expand on our observations that growth inhibition by EtOH-vescalagin can be rescued by iron supplementation (Figure 3.10) and that zincosome abundance is increased with EtOH-vescalagin treatment (Figure 3.19), we quantified intracellular levels of iron and zinc using atomic absorption spectroscopy (AAS). AAS is an established method to quantify metals in fluids including cells (Sunderman 1975). Zinc was first measured (Figure 3.20A). EtOHvescalagin significantly increased zinc levels by 18 and $20 \%$ in WT and zrt1 $\Delta$ compared to untreated control, respectively. Likewise, a treatment of $25 \mu \mathrm{M} \mathrm{ZnCl}_{2}$ resulted in significantly increased zinc levels by 38 and $51 \%$ in WT and zrtlA compared to untreated control, respectively. Iron was measured next (Figure 3.20B). EtOH-vescalagin significantly decreased iron levels by 49 and $37 \%$ in WT and zrt1 compared to untreated control, respectively. Likewise, a treatment of the iron chelator BPS resulted in significantly decreased iron levels by 69 and $60 \%$ in WT and zrt1 $\Delta$ compared to untreated control, respectively. These results confirm that EtOH-vescalagin increases intracellular zinc levels and decreases intraceullar iron levels. 
A

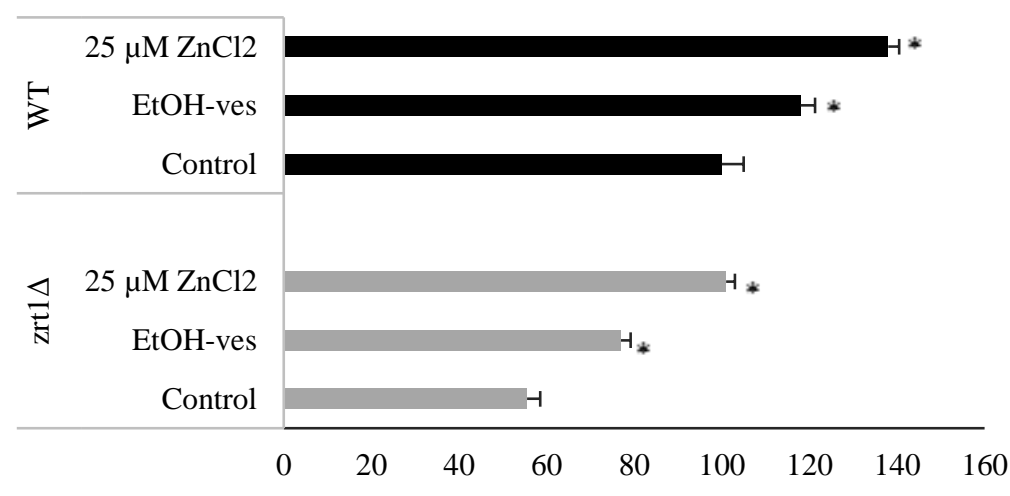

$\mathrm{B}$

Zinc abundance (\%)

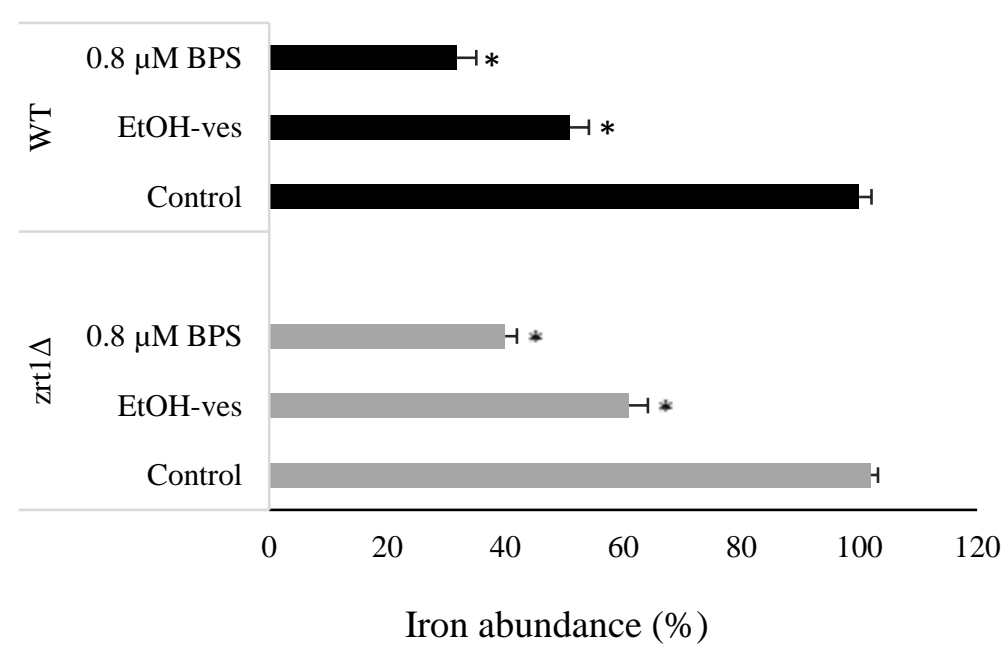

Figure 3-20. Zinc and iron quantification by AAS method in WT and zrt1A. Cells were treated with $305 \mathrm{nM}$ EtOH-vescalagin and two controls for zinc and iron $\left(25 \mu \mathrm{M} \mathrm{ZnCl}_{2}\right.$ and $\left.0.8 \mu \mathrm{M} \mathrm{BPS}\right)$ and collected at mid-log time point. Iron and zinc were quantified by furnace AAS method and data was shown in percentage compared to WT control. A) Zinc abundance. B) Iron abundance. *, $p<$ 0.05 , Student's $t$-test comparison with relative control.

\subsubsection{EtOH-vescalagin chelates iron}

The reduction in intracellular iron levels may be a consequence of iron chelation in the cell or in the media prior to uptake by the cell. To determine if EtOH-vescalagin chelates iron independent of cells, we tested the efficacy of EtOH-vescalagin to chelate iron from a complex of Ferrozin bound to iron (Figure 3.21A). The Ferrozin-iron complex forms a purple color, that upon treatment with the iron chelator control EDTA, turns into a clear solution in a dosedependent manner. Likewise, EtOH-vescalagin chelates the iron from the Ferrozine-iron 
complex in a dose-dependent manner. To quantify these visual observations, absorbance was measured using a spectrophotometer. The chelation activity of EtOH-vescalagin was 16.5, 38.3 and $58.4 \%$ with treatments of $600 \mathrm{nM}, 1 \mu \mathrm{M}$ and $1 \mathrm{mM}$, respectively. These values were not much different from the $19.8,47.7$ and $66.3 \%$ chelation activity with treatments of $600 \mathrm{nM}, 1$ $\mu \mathrm{M}$ and $1 \mathrm{mM}$ EDTA, respectively (Figure 3.21B). These results indicate that the mechanism of action of EtOH-vescalagin begins with the chelation of iron in the media, inducing a cellular response to low iron.

A
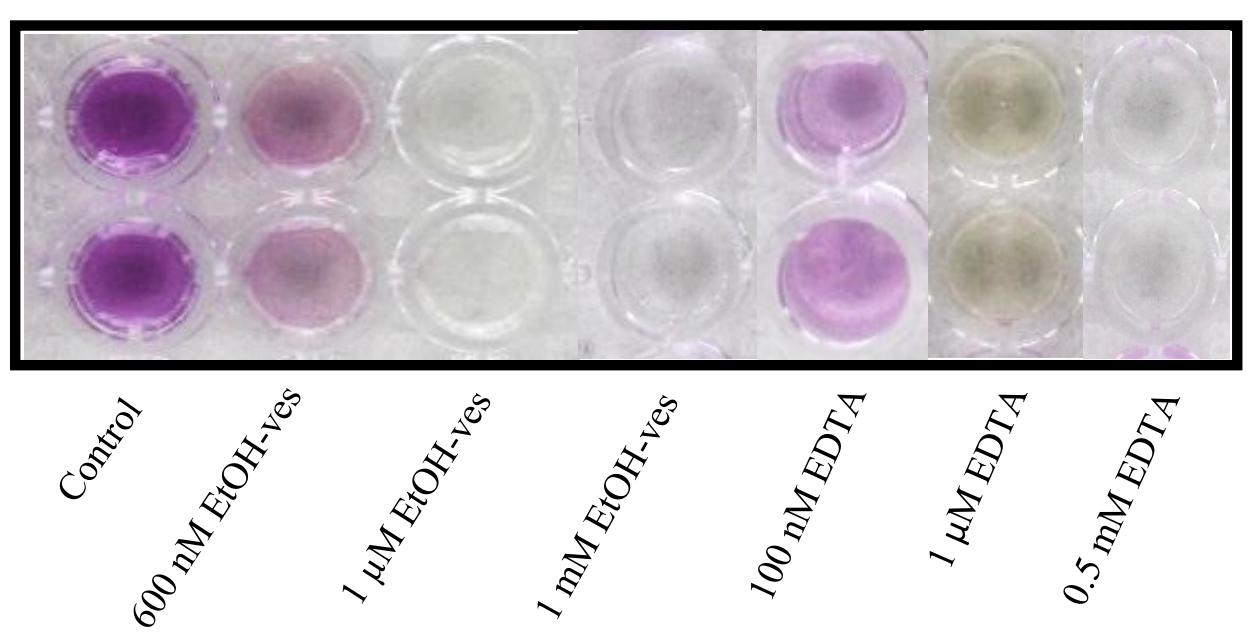

B

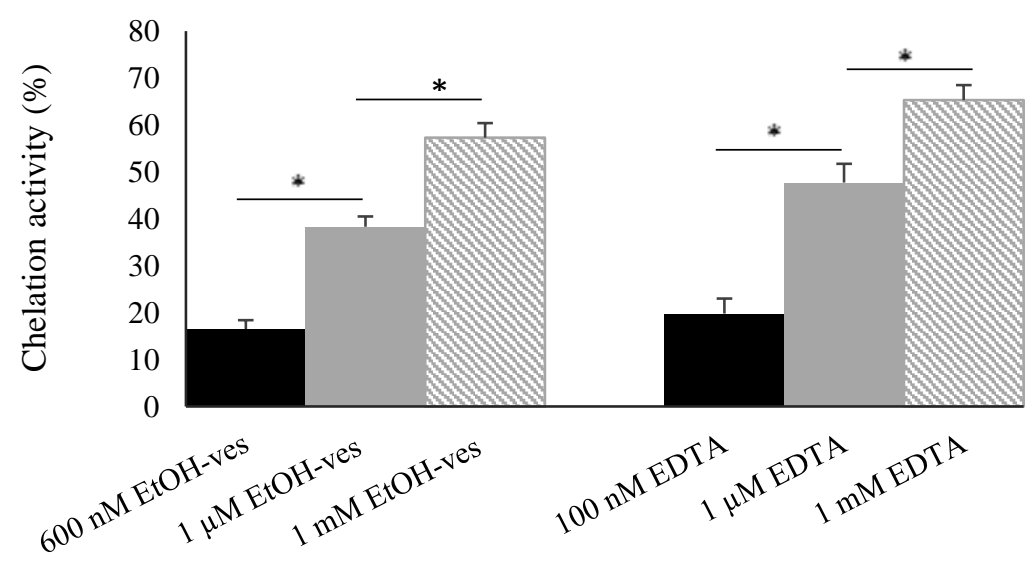

Figure 3-21. Ferrozin assay indicative of iron chelation activity of EtOH-vescalagin. A) Iron chelation assay with EtOH-vescalagin (EtOH-ves) and EDTA when the activity and color decrease is indicative of more chelation activity was measured by plate reader at OD of $562 \mathrm{~nm}$. B) Quantification of chelation activity with different concentrations of control and each treatment. Absorbance reading and images were taken after $10 \mathrm{~min}$. 


\subsubsection{EtOH-vescalagin increases expression of iron transporters}

Given our findings that iron supplementation rescued growth inhibition caused by EtOH-vescalagin (Figure 3.10) and that EtOH-vescalagin is an iron chelator (Figure 3.21), we predict a cell treated with EtOH-vescalagin will exhibit the molecular signatures of iron deficiency. Increased expression of high affinity iron transporters at the plasma membrane is an established cellular response to iron deficiency (Puig, et al. 2005). To assess expression of iron transporters, we exploited the yeast strains in the GFP library for which proteins are tagged with a GFP, thus fluorescence reflects endogenous levels and localization. Relative to RFP fluorescence of nuclei marked with mCherry and the cytosol marked with RedStar2, we evaluated the abundance of high affinity iron transporters at the plasma membrane (Fet3-GFP, Ftr1-GFP), low affinity iron transporter at the plasma membrane (Fet4-GFP), and an iron transporter at the vacuolar membrane (Smf3-GFP) in response to EtOH-vescalagin as well as an iron chelator control BPS (Figure 3.22). Upon treatment of $305 \mathrm{nM}$ EtOH-vescalagin (an $\mathrm{IC}_{20}$ concentration), levels of Fet3-GFP, Ftr1-GFP and Smf3-GFP were increased by $38 \%, 54 \%$ and $62 \%$, respectively, compared to the untreated control (Figure 3.23). This was in contrast to the $18 \%$ decrease in expression that was detected in the low affinity iron transporter Fet4-GFP, possibly since the intracellular zinc level in EtOH-vescalagin treated cells compared to control cells. For comparison, an iron chelator BPS treatment ( $\mathrm{IC}_{20}$ concentration) increased levels of Fet3-GFP, Ftr1-GFP, Fet4-GFP and Smf3-GFP by 66, 41, 70 and 31, respectively (Figure 3.23). Increased expression of Fet4-GFP with BPS but not EtOH-vescalagin suggest that EtOHvescalagin has more targets than iron chelation alone. These results suggest that increased expression of iron transporters is a means to compensate for the reduced levels of iron in the cell. 


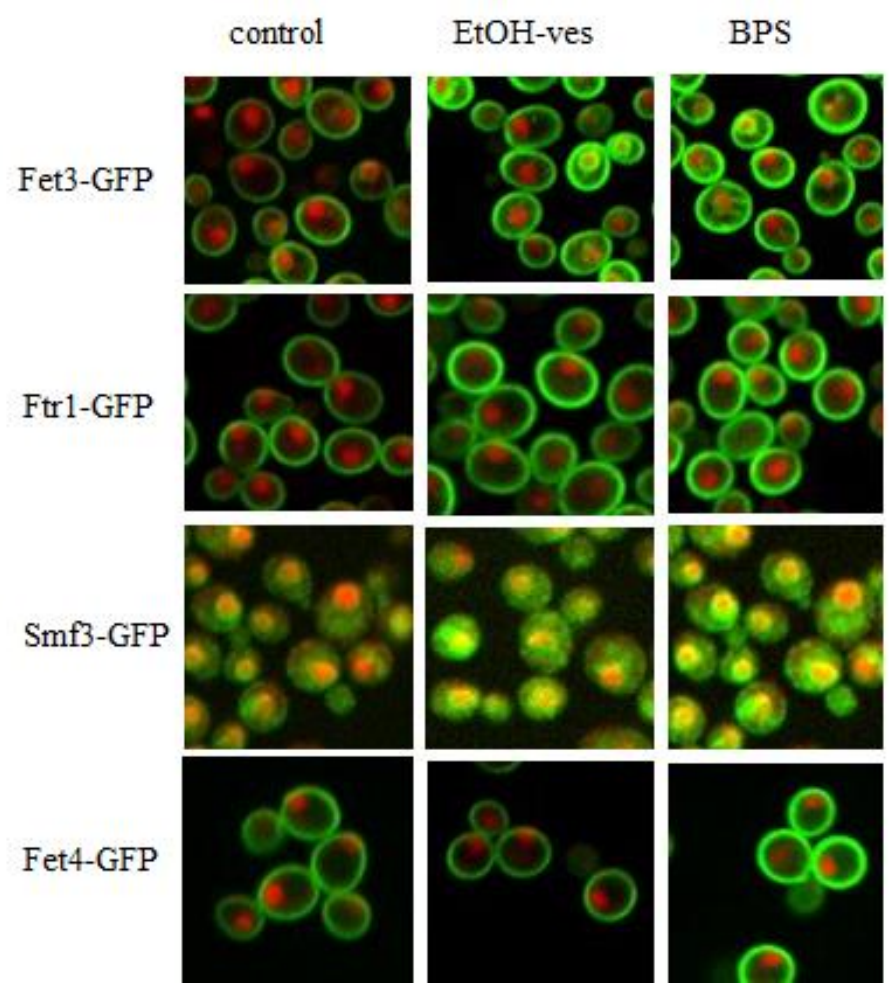

Figure 3-22. Expression level of iron transporters in presence of EtOH-vescalagin and BPS. GFP protein shows protein expression abundance of each iron transporter in presence of $0.8 \mu \mathrm{M}$ BPS and EtOH-vescalagin. Images were taken by Opera fluorescent microscope (Perkin Elmer) after 6 hour treatment with EtOH-vescalagin.

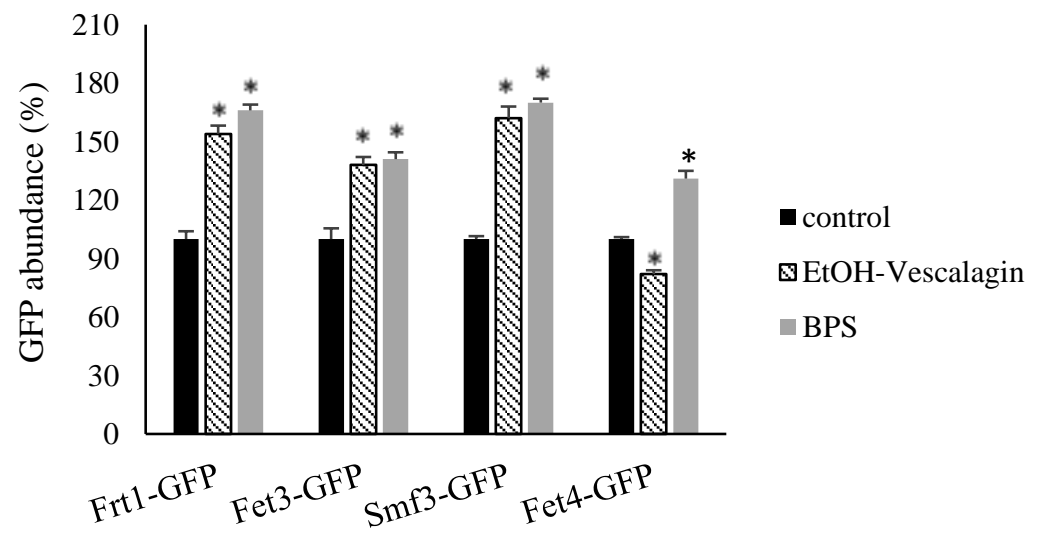

Figure 3-23. Quantification of GFP protein abundance of iron transporters in presence of EtOH-vescalagin and BPS. The GFP-image analysis was done using ACAPELLA. *, $p<0.05$, Student's $t$-test comparison with untreated control. 


\subsubsection{EtOH-vescalagin increases expression of vacuolar zinc transporter and decreases expression of plasma membrane zinc transporter}

Since EtOH-vescalagin increased intracellular levels of zinc (Figure 3.19 and 3.20), we predict a cell treated with EtOH-vescalagin will exhibit the molecular signatures of zinc toxicity. Increased expression of transporters importing zinc into the vacuole is an established means for a cell to store excess zinc in the vacuole (MacDiarmid, et al. 2000). To assess expression of the proteins regulating import of zinc, we evaluated abundance of Zrc1-GFP and Cot1-GFP at the vacuolar membrane as well as the high affinity zinc transporter Zrt1-GFP at the plasma membrane in response to EtOH-vescalagin. We also evaluated the levels of these proteins in response to the iron chelator BPS to further discern the effects of iron chelation on zinc metabolism. Relative to the untreated control, the Cot1-GFP was increased by $77 \%$ and the Zrt1-GFP was decreased by $48 \%$ upon treatment with $305 \mathrm{nM} \mathrm{EtOH-vescalagin} \mathrm{(Figure}$ 3.24). No change in expression was detected in the other vacuolar membrane transporter Zrc1GFP, possibly since the increased expression of Cot1-GFP is the primary means of importing zinc into the vacuole. For comparison, the iron chelator BPS elicited a different effect as EtOHvescalagin with increased expression of Cot1-GFP by $98 \%$ and Zrt1-GFP by $46 \%$ compared to untreated controls (Figure 3.25), results that are consistent with increased COT1 expression as a protective mechanism to either sequester metals (zinc and cobalt) in the vacuole in response to iron deficiency (Philpott and Protchenko 2008) or store zinc in response to zinc toxicity (Simm, et al. 2007). These results indicate that increased expression of Cot1-GFP at the vacuolar membrane combined with decreased expression of Zrt1-GFP at the plasma membrane are mechanisms by which cells treated with EtOH-vescalagin respond to zinc toxicity. 


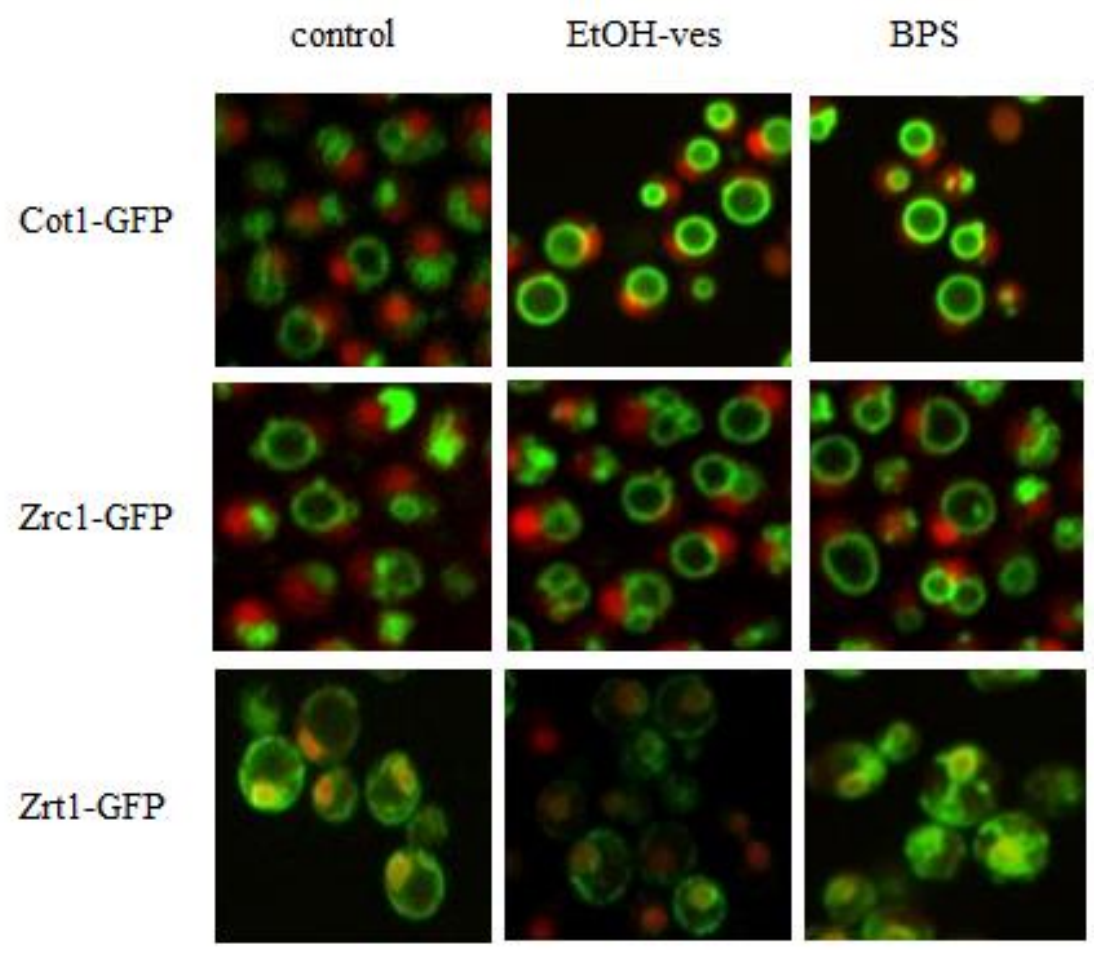

Figure 3-24. Expression level of zinc transporters in presence of $305 \mathrm{nM} \mathrm{EtOH-vescalagin} \mathrm{and}$ BPS. Zinc transporters (Cot1p, Zrc1p, Zrt1p) were monitored in presence of $305 \mathrm{nM} \mathrm{EtOH-vescalagin}$ after 6 hours treatment in $30^{\circ} \mathrm{C}$. BPS was used at $0.8 \mu \mathrm{M}$ for comparison. The GFP tagged strains were grown in YPD agar plate for 14 hours and then inoculated to the 384 Opera plate with/without EtOH-vescalagin

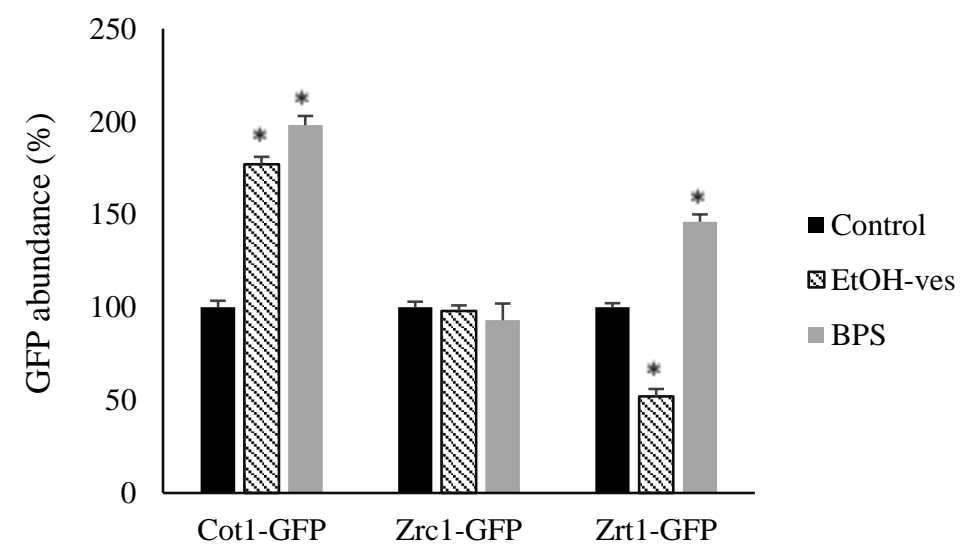

Figure 3-25. Quantification of protein abundance of zinc transporters in presence of EtOHvescalagin and BPS. (305 nM EtOH-ves and $0.8 \mu \mathrm{M}$ BPS). Data were analysed from Opera images using ACAPELLA and median value of triplicate was monitored \pm SD. ${ }^{*}, p<0.05$, Student's $t$-test comparison with relative control. 


\subsubsection{EtOH-vescalagin disrupts retromer function}

Since deletion strains of retromer subunits were sensitive to EtOH-vescalagin (Figure 3.5), we predict EtOH-vescalagin will impact either abundance or localization of these retromer subunit proteins. It has been reported that the levels and localization of VPS10 are responsive to disruptions in VPS29 or VPS35 (Seaman, et al. 1997). To assess expression and localization of the retromer complex, we monitored the fluorescence of representative retromer subunits that are represented in the GFP library (Vps5-GFP, Vps10-GFP, Vps17-GFP, Vps26-GFP) in response to EtOH-vescalagin. Relative to the untreated control, the expression levels of VPS5GFP, Vps10-GFP, Vps17-GFP, Vps26-GFP were increased by 17, 27, 24 and 28\%, respectively, upon treatment with $305 \mathrm{nM}$ EtOH-vescalagin (Figure 3.26). Similar levels of increased expression were observed in Vps5-GFP, Vps10-GFP, Vps17-GFP and Vps26-GFP upon treatment with $0.8 \mu \mathrm{M}$ BPS treatment with increases of $20,38,24$ and $35 \%$, respectively. In addition to increased expression, the EtOH-vescalagin treatment altered the localization of these three proteins from endosomes to the vacuolar membrane. We also evaluated the levels of these proteins in response to the iron chelator BPS to further discern the effects of iron chelation on the retromer complex (Figure 3.27). The BPS treatment phenocopied the EtOHvescalagin treatment with increased expression and relocalization from endosomes to vacuolar membrane. These results are consistent with the mislocalization of Vps10-GFP from endosomes to the vacuolar membrane upon disrupted retromer function (Seaman, et al. 1997) (Burda, et al. 2002) (Arlt, et al. 2015), confirming that EtOH-vescalagin impacts retromer function and that the retromer mislocalization phenotypes by EtOH-vescalagin are a consequence of iron chelation. 


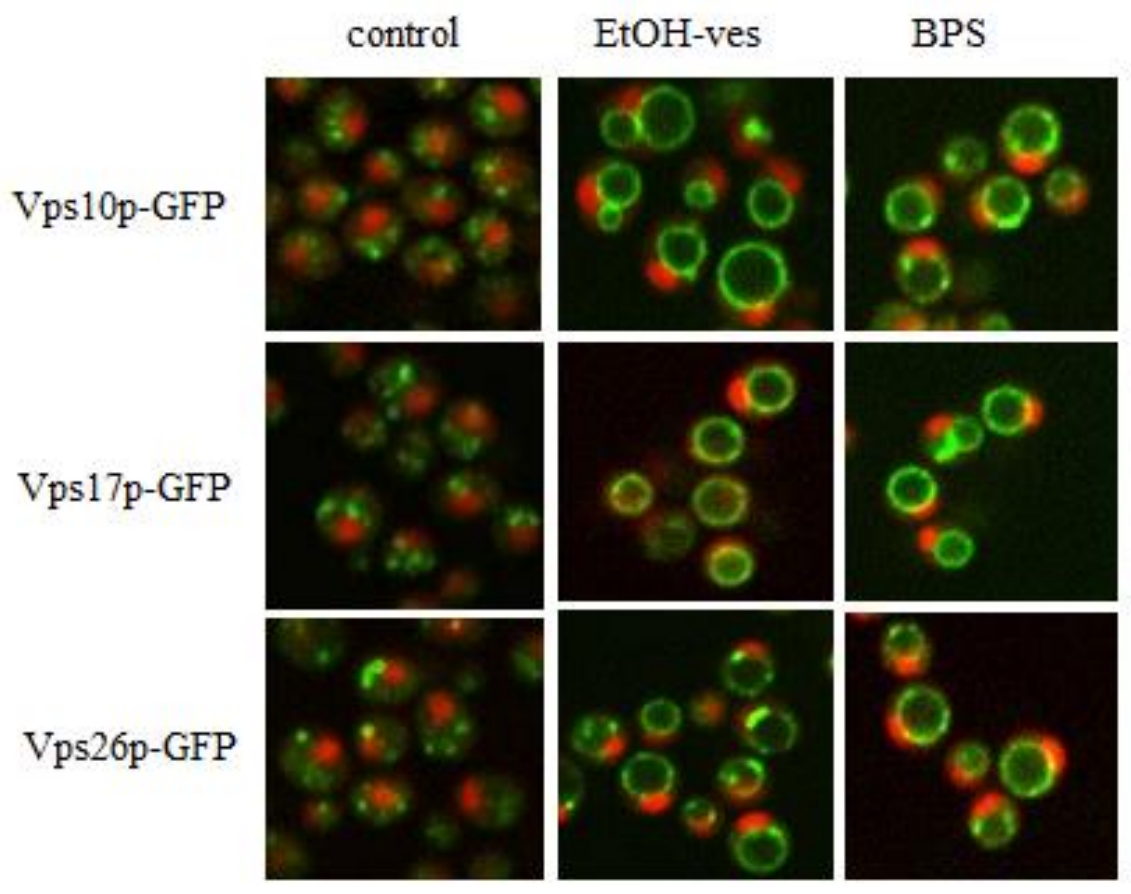

Figure 3-26. Mislocalization and expression level of retromer subunits in presence of EtOHvescalagin and BPS. $305 \mathrm{nM}$ EtOH-ves and $0.8 \mu \mathrm{M}$ BPS were used. GFP images were taken by Opera fluorescent microscope after 6 hour treatment with EtOH-vescalagin in 384-well plate.

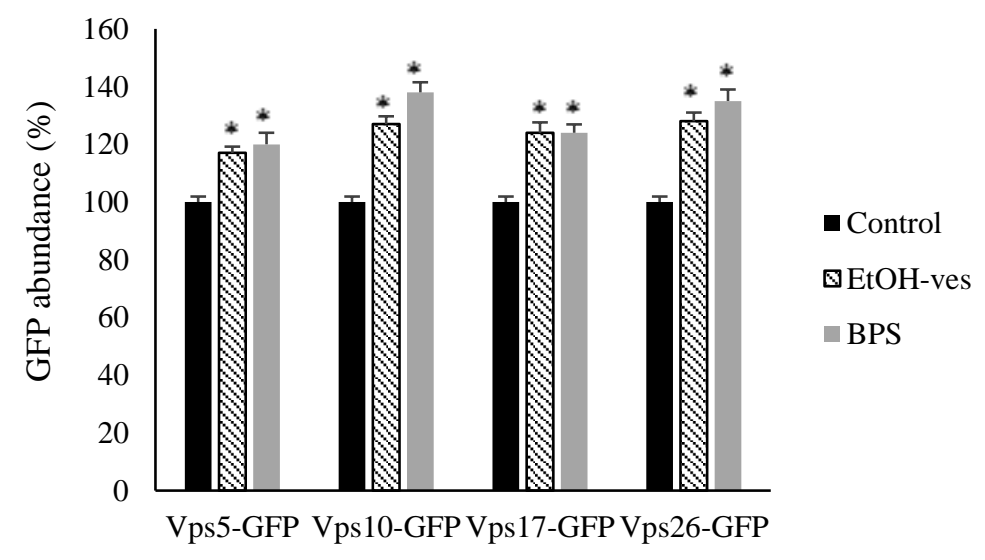

Figure 3-27. Quantification of GFP-protein abundance of retromer proteins in presence of EtOH-vescalagin and BPS. The GFP-image analysis was done by ACAPELLA from Opera result in previous figure and average mean value was calculated $\pm \mathrm{SD}$. ${ }^{*}, p<0.05$, Student's $t$-test comparison with relative control. 


\subsubsection{Deletion-based GFP analyses strengthen the iron-zinc-retromer mechanisms of EtOH-vescalagin}

We next sought to further investigate the bioactivity of EtOH-vescalagin that includes iron chelation, zinc toxicity and disrupted retromer function. Thus far we have shown that expression levels and/or localization of proteins involved in iron transport, zinc transport and retromer function are altered with EtOH-vescalagin treatment in control yeast (WT background). To investigate the interactions of these processes, we examined the abundance and localization of proteins in these processes in the background of gene deletions. Using the methodology of a Synthetic Genetic Array (Tong, et al. 2001), we generated double mutants to examine the impact of EtOH-vescalagin on GFP-tagged zinc and retromer proteins in the background of iron gene deletions, GFP-tagged iron and retromer proteins in the background of zinc gene deletions, and GFP-tagged iron and zinc proteins in the background of retromer gene deletions.

\subsubsection{EtOH-vescalagin regulates expression of retromer subunits and zinc transporters independent of iron transporters}

The iron transport gene deletion strains (fet3 $\Delta$, fet4 4 , smf $3 \Delta$, fre $2 \Delta$ ) were crossed with GFP-tagged strains representing the retromer complex (Vps5-GFP, Vps10-GFP, Vps17-GFP, Vps26-GFP), zinc transport into the cell at the plasma membrane (Zrt1-GFP), and zinc transport into the vacuole at the vacuolar membrane (Cot1-GFP). Localization and abundance of the GFP-tagged proteins in the deletion strains was then examined (Figure 3.28A). The expression levels of all four retromer proteins in all four iron transport gene deletion strains were significantly increased in response to EtOH-vescalagin. The localization of Vps10-GFP, Vps17-GFP and Vps26-GFP was altered from endosomes to the vacuolar membrane. The expression level of Zrt1-GFP was decreased while the expression level of Cot1-GFP was 
increased. These results are consistent with the abundance and localization of these proteins in a WT background, suggesting that iron transport genes are not required for the upregulation and mislocalization of retromer subunits as well as altered expression of zinc transporters (Figure 3.28C).

To decipher EtOH-vescalagin from iron chelation and simultaneously evaluate how much of these results are due to the iron chelation mechanism of EtOH-vescalagin, we treated cells with iron chelator BPS (Figure 3.28B). Like EtOH-vescalagin, the BPS treatment increased expression of Vps5-GFP, Vps10-GFP, Vps17-GFP and Vps26-GFP, with the additional effect of altering the localization of the latter three proteins from the endosomes to the vacuolar membrane. Also similar with EtOH-vescalagin was the increased expression of Cot1-GFP with BPS treatment. However, there was a major distinction in that the BPS treatment resulted in increased expression of Zrt1-GFP, a result in contrast with the EtOHvescalagin treatment that decreased expression of Zrt1-GFP. These opposing effects on the high affinity zinc transporter ZRT1 suggest that BPS treatment induces a state of zinc-deficiency, while EtOH-vescalagin induces a state of zinc toxicity. 
A

B

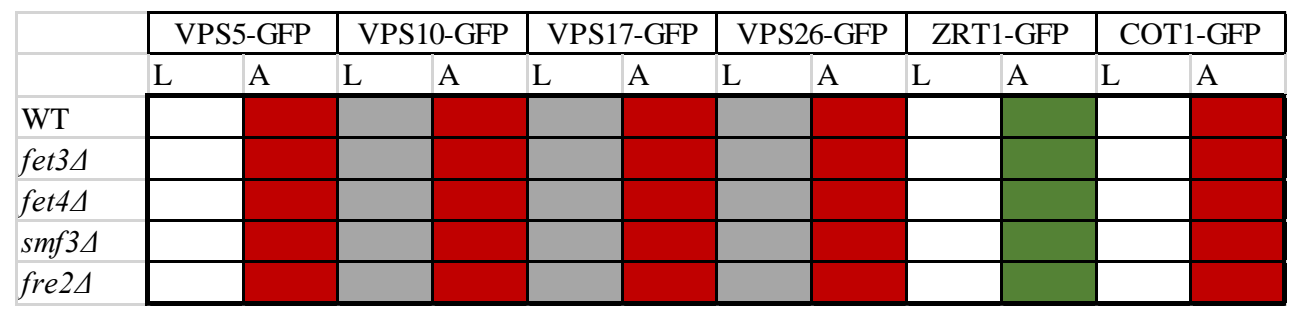

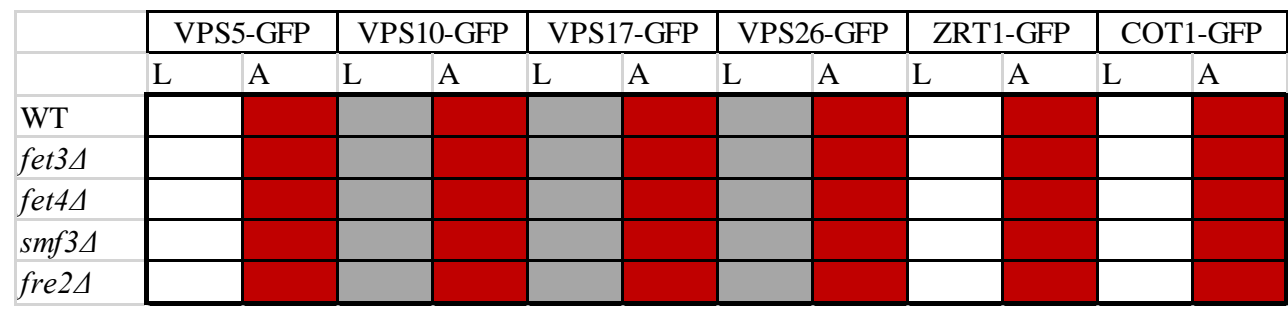

$\mathrm{C}$

\begin{tabular}{|l|}
\hline decreased expression \\
\hline increased expression \\
\hline mislocalization from endosome to vacuole membrane \\
\hline
\end{tabular}

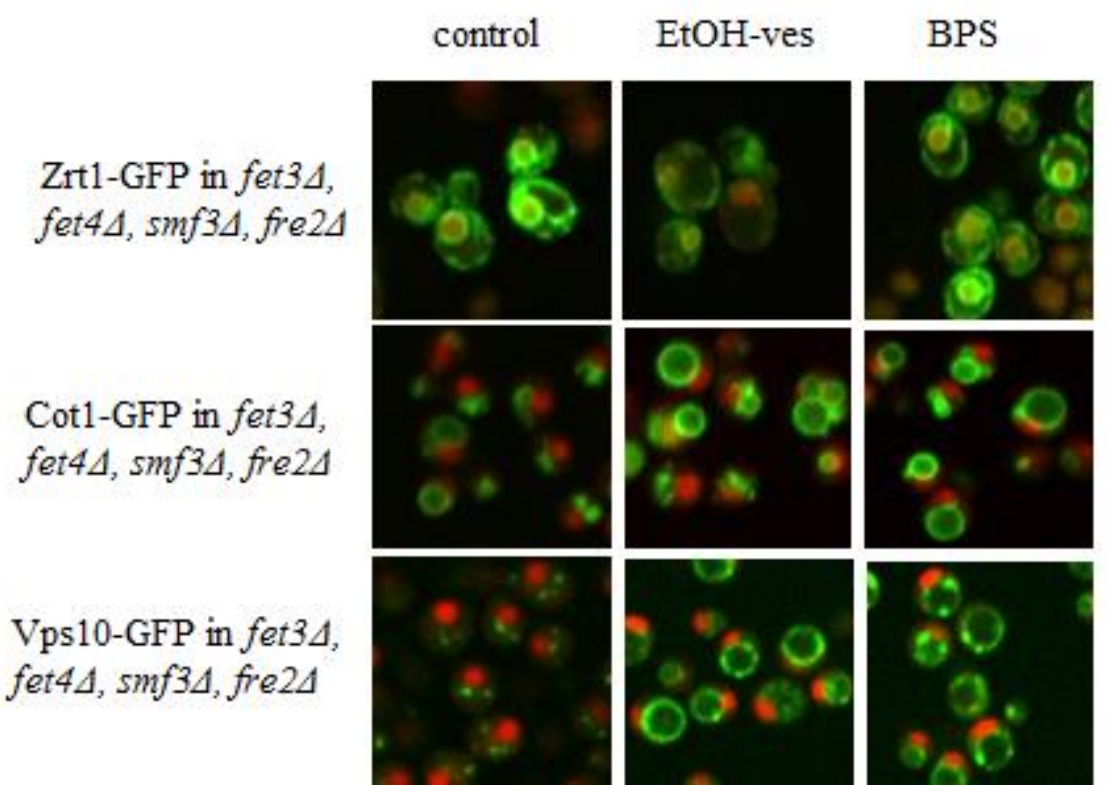

Figure 3-28. Retromer and zinc transporter expressions in iron deletion strains fet3A, fet4A, smf34, fre2A strains with different zinc and retromer GFP proteins. Simplified heat map of protein localisation (L) and abundance (A) in different deletion strains with A) EtOH-vescalagin and B) with BPS. C) Representative images of phenotypes in the heat map. 


\subsubsection{EtOH-vescalagin regulates expression of retromer subunits and iron}

\section{transporters independent of zinc transporters}

The zinc transport gene deletion strains $(z r t 1 \Delta, z r t 2 \Delta, z r c 1 \Delta$, cot1 $1 \Delta)$ were crossed with GFP-tagged strains representing the retromer complex (Vps5-GFP, Vps10-GFP, Vps17-GFP, Vps26-GFP) and iron transport (Ftr1-GFP, Fet3-GFP, Fet4-GFP, Smf3-GFP, Fre2-GFP). Localization and abundance of the GFP-tagged proteins in the deletion strains was then examined (Figure 3.29). The expression levels of all four retromer proteins were significantly increased in response to EtOH-vescalagin in WT, zrt2A, zrc1 , and $\cot 1 \Delta$; however, this change was not seen in zrt1 $\Delta$. The EtOH-vescalagin also resulted in increased expression of Ftr1-GFP, Fet3-GFP, Smf3-GFP, Fre2-GFP in all deletion strains consistent with results in WT. In contrast, the expression levels of Fet4-GFP were decreased in all strains but zrt1A in which it was increased, while Fet4-GFP was not altered in zrt2 4 . In addition, EtOH-vescalagin altered the localization of Ftr1-GFP, Fet3-GFP and Fet4-GFP from the plasma membrane to the vacuole only in zrt1A (Figure 3.29A). With the exception of $z r t 1 \Delta$, these results are consistent with the abundance and localization of these proteins in a WT background, suggesting that either zinc deficiency in zrt1 $\Delta$ prevents the zinc toxicity-causing mechanism or alternatively that ZRT1 is required to induce the zinc toxicity mechanism through iron homeostasis and retromer recycling.

A

\begin{tabular}{l|l|l|l|l|l|l|l|l|l|l|l|l|l|l|l|l|l|l|}
\hline \multicolumn{1}{l|}{} & VPS5-GFP & VPS10-GFP & VPS17-GFP & VPS26-GFP & FTR1-GFP & FET3-GFP & FET4-GFP & SMF3-GFP & FRE2-GFP \\
\hline
\end{tabular}

$\mathrm{B}$

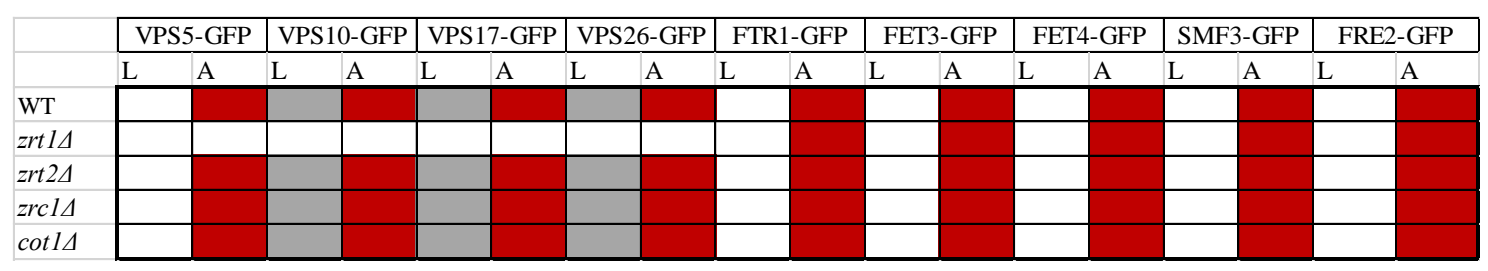


mislocalization from endosome to vacuole membrane

mislocalization from plasma membrane to vacuole

decreased expression

increased expression

C

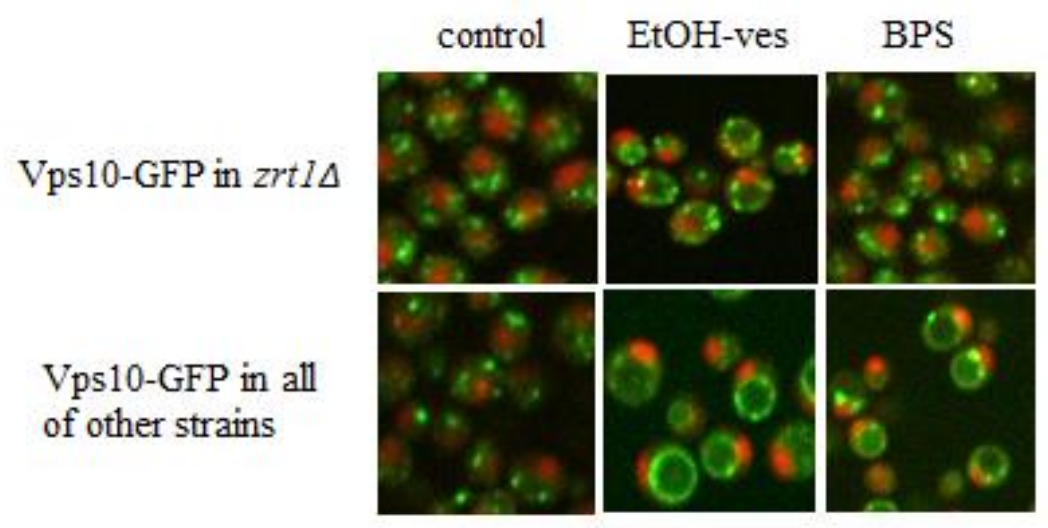

D

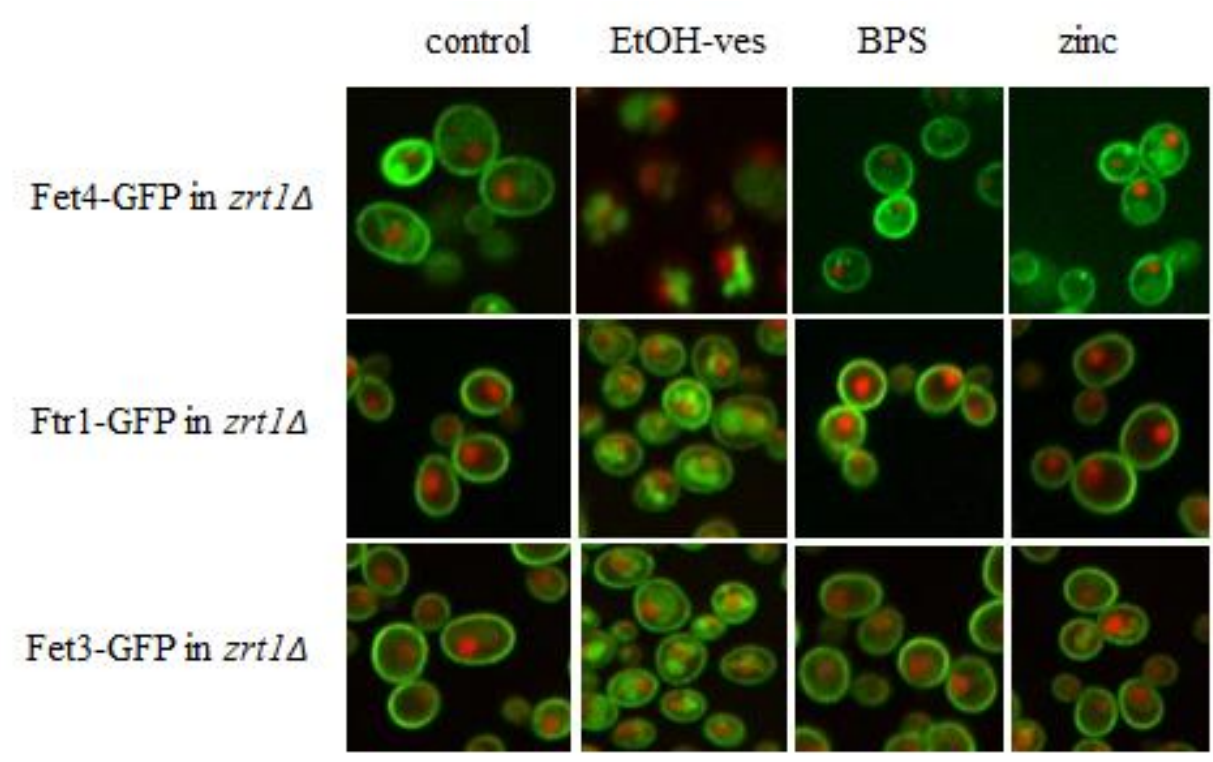

Figure 3-29. Retromer and iron transporters expression in zinc deletion strains. Simplified heat map of protein localisation (L) and abundance (A) in different deletion strains with A) EtOHvescalagin and B) with BPS. C) Representative images of VPS10p-GFP expression. D) Iron transporters expressions in zrt1A. 
To decipher EtOH-vescalagin from iron chelation and simultaneously evaluate how much of these results are due to the iron chelation mechanism of EtOH-vescalagin, we treated cells with iron chelator BPS (Figure 3.29B). Like EtOH-vescalagin, the BPS treatment increased expression levels and localization of the retromer subunits in all strains except zrt1 $\Delta$. The BPS treatment increased expression of all iron transport proteins in all deletion strains; this result was distinguished from EtOH-vescalagin that decreased expression of Fet4-GFP in most strains. In addition, the BPS treatment did not alter the localization of Ftr1-GFP, Fet3-GFP and Fet4-GFP in zrt1A. To determine the contribution of zinc to the EtOH-vescalagin and BPS phenotypes, we treated cells with $25 \mu \mathrm{M} \mathrm{ZnCl} 2$ and determined that the Fet4-GFP, Ftr1-GFP and Fet3-GFP phenotypes in the zinc treatment resembled the BPS treatment more than the EtOH-vescalagin treatment. These distinct effects of EtOH-vescalagin and BPS indicate that the mechanism of action of EtOH-vescalagin includes more than iron chelation, such as disrupted zinc homeostasis and retromer recycling.

Lastly, EtOH-vescalagin treatment resulted in the mislocalization of Vps10-GFP from endosomes into the vacuolar membrane in WT and all deletion strains except zrtlA (Figure 3.29C). Interestingly, Vps10-GFP also remained in the endosomes with BPS treatment in zrt1 4 . This result suggests either low levels of zinc can rescue the VPS10 function defect (or VPS26/VPS29/VPS35 defects caused VPS10 mislocalization) or ZRT1 has an indirect role in retromer regulation.

\subsubsection{EtOH-vescalagin mislocalizes Fet4-GFP in retromer deletion strains}

The retromer subunit gene deletion strains (vps54, vps104, vps174, vps264, vps294, vps354) were crossed with GFP-tagged strains representing iron transport (Ftr1-GFP, Fet3GFP, Fet4-GFP, Smf3-GFP, Fre2-GFP), zinc transport into the cell at the plasma membrane (Zrt1-GFP), and zinc transport into the vacuole at the vacuolar membrane (Cot1-GFP). 
Localization and abundance of the GFP-tagged proteins in the deletion strains was then examined (Figure 3.30). There was one pronounced localization change in which Fet4-GFP was mislocalized from the plasma membrane to the vacuole in all retromer deletion strains (Figure 3.30A). Zrt1-GFP was also increased in retromer deletion strains while it was decreased in WT in presence of EtOH-vescalagin.

To decipher EtOH-vescalagin from iron chelation and simultaneously evaluate how much of these results are due to the iron chelation mechanism of EtOH-vescalagin, we treated cells with iron chelator BPS (Figure 3.30B). The BPS treatment increased expression levels of iron and zinc proteins, and unlike the EtOH-vescalagain treatment, Fet4-GFP localization was not altered with BPS treatment. To determine the contribution of zinc to the EtOH-vescalagin and BPS phenotypes, we treated cells with $25 \mu \mathrm{M} \mathrm{ZnCl}_{2}$ and determined that the Fet4-GFP mislocalization from the plasma membrane to the vacuole occurred with the zinc treatment. These results indicate that retromer function mediates localization of Fet4-GFP in cells treated with EtOH-vescalagin, and that this mislocalization is more a consequence of zinc than iron chelation (Figure 3.30D). 
A

B

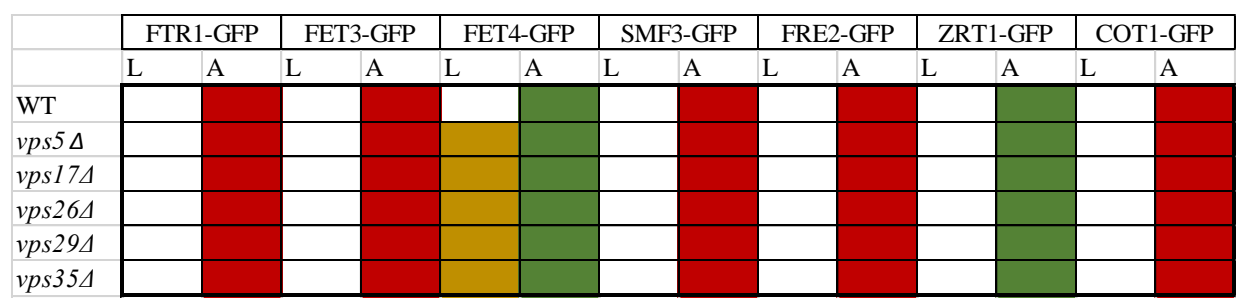

\begin{tabular}{|c|c|c|c|c|c|c|c|}
\hline & FTR1-GFP & FET3-GFP & FET4-GFP & SMF3-GFP & FRE2-GFP & ZRT1-GFP & COT1-GFP \\
\hline & $\begin{array}{lll}\mathrm{L} & \mathrm{A}\end{array}$ & $\begin{array}{ll}\mathrm{L} & \mathrm{A}\end{array}$ & $\begin{array}{ll}\mathrm{L} & \mathrm{A}\end{array}$ & $\begin{array}{ll}\mathrm{L} & \mathrm{A} \\
\end{array}$ & $\begin{array}{ll}\mathrm{L} & \mathrm{A} \\
\end{array}$ & \begin{tabular}{l|l}
$\mathrm{L}$ & $\mathrm{A}$ \\
\end{tabular} & $\begin{array}{ll}\mathrm{L} & \mathrm{A} \\
\end{array}$ \\
\hline \multicolumn{8}{|l|}{ WT } \\
\hline vps5 $\Delta$ & & & & & & & \\
\hline vps 174 & & & & & & & \\
\hline vps 264 & & & & & & & \\
\hline vps294 & & & & & & & \\
\hline$v p s 354$ & & & & & & & \\
\hline
\end{tabular}

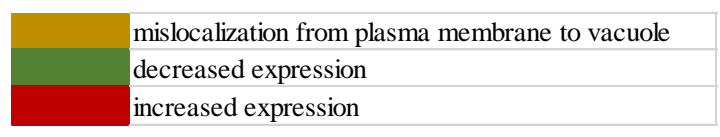

$\mathrm{C}$

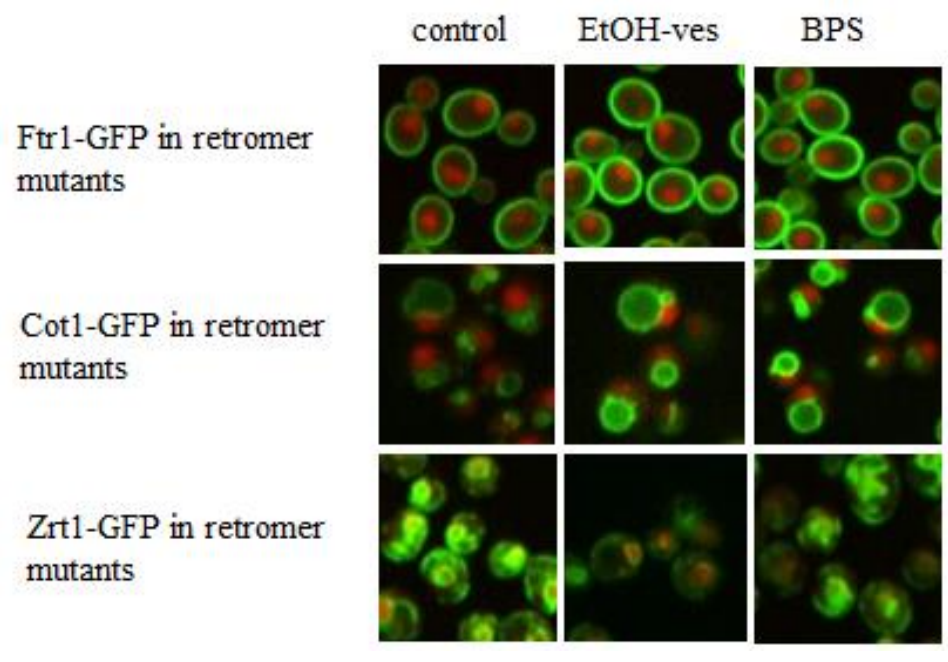

$\mathrm{D}$

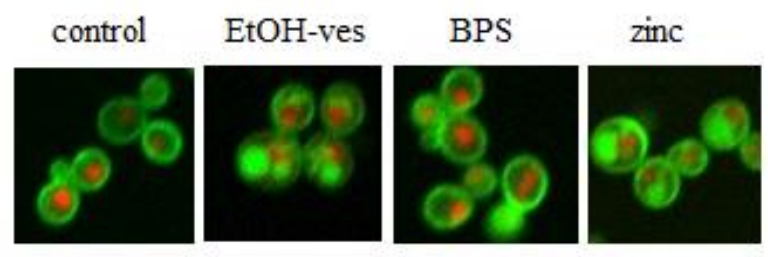

Figure 3-30. Iron and zinc transporters expression in retromer deletion strain. Simplified heat map of protein localisation (L) and abundance (A) in different deletion strains with A) EtOHvescalagin and B) with BPS. C) Representative images of iron and zinc transporters expression in heat map. D) Fet4-p mislocalization in retromer deletion strains. 


\subsubsection{Overexpression analyses strengthen the iron-zinc-retromer mechanisms of EtOH-vescalagin}

We next sought to determine the extent to which the mechanisms of action of EtOHvescalagin are interactive in the processes of iron chelation, zinc toxicity and disrupted retromer function. Thus far, we have shown significant growth defects for deletion strains in these processes, thus it will be informative to quantify the extent by which overexpression of genes in each process affects sensitivity. To investigate the effect of overexpression, we transformed overexpression plasmids into deletion strains and compared growth in the presence and absence of EtOH-vescalagin on media containing galactose (induced overexpression of gene on plasmid) with growth on media containing glucose (repressed expression of gene on plasmid).

\subsubsection{Overexpression of iron, zinc transporters and retromer subunits regulates sensitivity to EtOH-vescalagin in WT}

For a baseline assessment of overexpression, we transformed galactose-inducible overexpression plasmids of iron transporters (FTR1, FRE2, SMF3), zinc transporters (ZRT1, ZRT2, ZRC1), and main retromer subunits (VPS26, VPS29, VPS35) in WT. Relative to 35\% inhibition caused by EtOH-vescalagin in WT expressing an empty vector, ovexpression of genes involved in iron metabolism, zinc metabolism and retromer recycling altered this sensitivity. Overexpression of the iron transporters FTR1, SMF3 and FRE2 rescued the EtOHvescalagin inhibition by $20 \%, 16 \%$ and $32 \%$, respectively, compared to untreated cells (Figure 3-31). This result confirms our previous findings stating EtOH-vescalagin reduces intracellular iron levels. Overexpression of the high affinity zinc transporter ZRT1 exacerbated the growth defect by $30 \%$, reiterating that the EtOH-vescalagin induces zinc toxicity that is only worsened with increased uptake of zinc from the media. Overexpression of the retromer subunits VPS26, 
VPS29 and VPS35 ameliorated sensitivity by 16, 20 and 17\%, respectively. These results further demonstrate that EtOH-vescalagin sensitivity is regulated by genes in the processes of iron metabolism, zinc metabolism and retromer recycling.

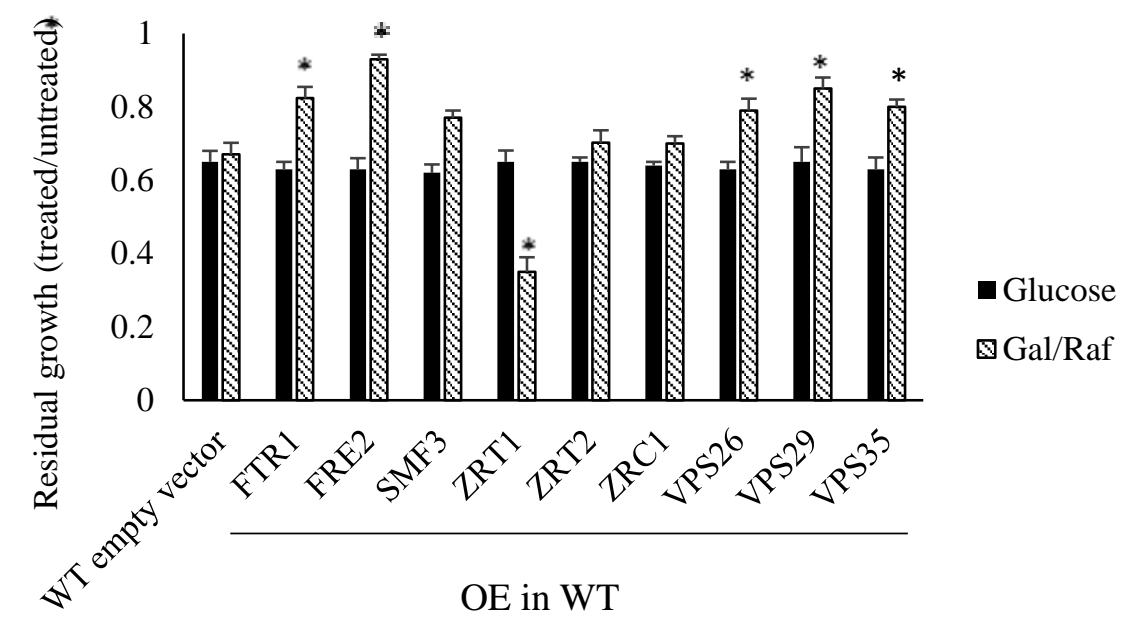

Figure 3-31. Overexpression of iron, zinc transporters and retromer subunits regulates sensitivity to EtOH-vescalagin in WT. Effect of overexpression on growth was measured on glucose (control) and 2\% Galactose/1\% Raffinose with/without $390 \mathrm{nM}$ EtOH-vescalagin. *, $p<0.05$, Student's t-test comparison with relative control.

\subsubsection{Overexpression of zinc transporters exacerbates sensitivity of iron transporter deletions to EtOH-vescalagin}

To investigate overexpression of zinc and retromer genes in iron transporter deletion strains, we transformed galactose-inducible overexpression plasmids of zinc transporters (ZRT1, ZRT2), and retromer subunits (VPS26, VPS29, VPS35) in ftr1 $\Delta$ and fet4A. In the background of either the deletion of the high affinity transporter FTR1 or the low affinity transporter FET4, no changes in EtOH-vescalagin sensitivity were observed with overexpression of retromer subunits (Figure 3-32 and Figure 3-33). These results suggest that iron deficiency is upstream of retromer dysfunction based on the inability to alter EtOHvescalagin sensitivity without the iron transporters. In contrast, overexpression of the high and low affinity zinc transporters ZRT1 and ZRT2 exacerbated the sensitivity of fet4A to EtOH- 
vescalagin by $11-14 \%$. These results further demonstrate that the iron chelator $\mathrm{EtOH}$-vescalagin sensitivity is a consequence of iron depletion and zinc toxicity (in this caused by overexpression of zinc transporters in the fet4 $\Delta$ strain).

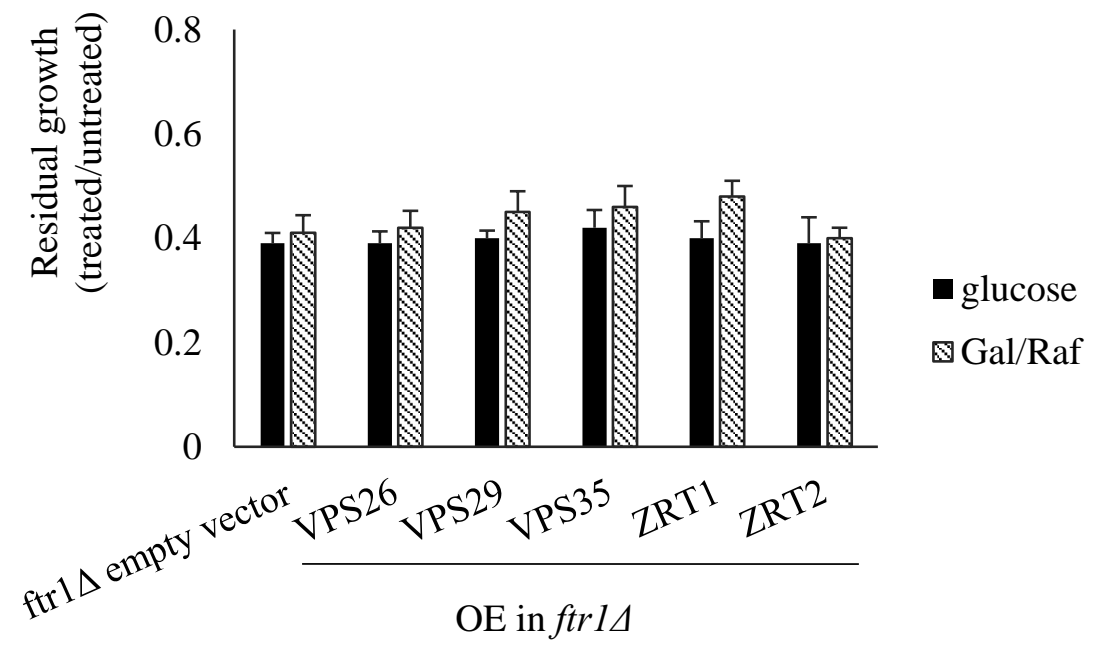

Figure 3-32. Overexpression of zinc transporters and retromer subunits in ftr1A strain. Effect of overexpression on growth was measured on glucose (control) and 2\% Galactose/1\% Raffinose with/without $390 \mathrm{nM} \mathrm{EtOH-vescalagin.} \mathrm{*,} p<0.05$, Student's $t$-test comparison with relative control.

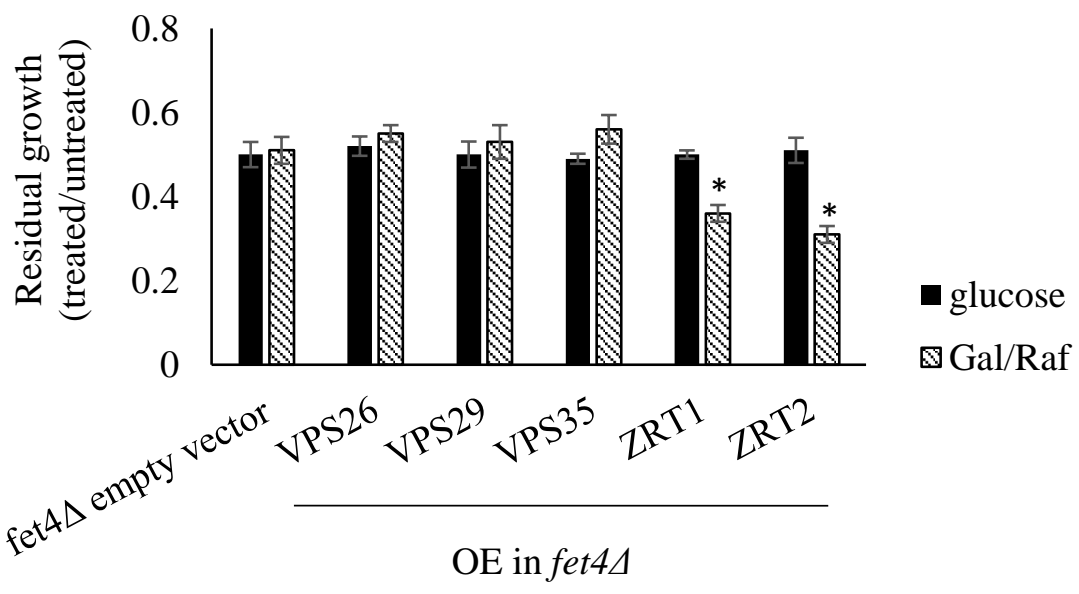

Figure 3-33. Overexpression of zinc transporters exacerbates sensitivity of fet4 $\Delta$ to EtOH-vescalagin. Effect of overexpression on growth was measured on glucose (control) and 2\% Galactose/1\% Raffinose with/without $390 \mathrm{nM} \mathrm{EtOH-vescalagin.} \mathrm{*,p}$ $<0.05$, Student's $t$-test comparison with relative control. 


\subsubsection{Overexpression of iron transporters and retromer subunits regulates sensitivity of zinc transporter deletions to EtOH-vescalagin}

To investigate overexpression of iron and retromer genes in zinc transporter deletion strains, we transformed galactose-inducible overexpression plasmids of iron transporters (FTR1, FRE2) and retromer subunits (VPS26, VPS29, VPS35) in zrt1A (zinc deficiency), zrt2 (zinc deficiency) and zrc1 $($ (zinc toxicity). In the background of either zinc deficiency (zrt1 $\Delta$, $z r t 24)$ or zinc toxicity $(z r c 14)$, overexpression of the iron reductase FRE2 rescued sensitivity by 24-34\% (Figure 3-34 and Figure 3-35). In addition, FTR1 overexpression decreased sensitivity in zrc1 (Figure 3-36). Impressively, overexpression of retromer subunits increased EtOH-vescalagin sensitivity in the condition of zinc-deficiency $(z r t 1 \Delta, z r t 2 \Delta)$ and decreased EtOH-vescalagin sensitivity in the condition of zinc-toxicity (zrclA). These results further demonstrate that EtOH-vescalagin sensitivity is a consequence of retromer function and zinc homeostasis.

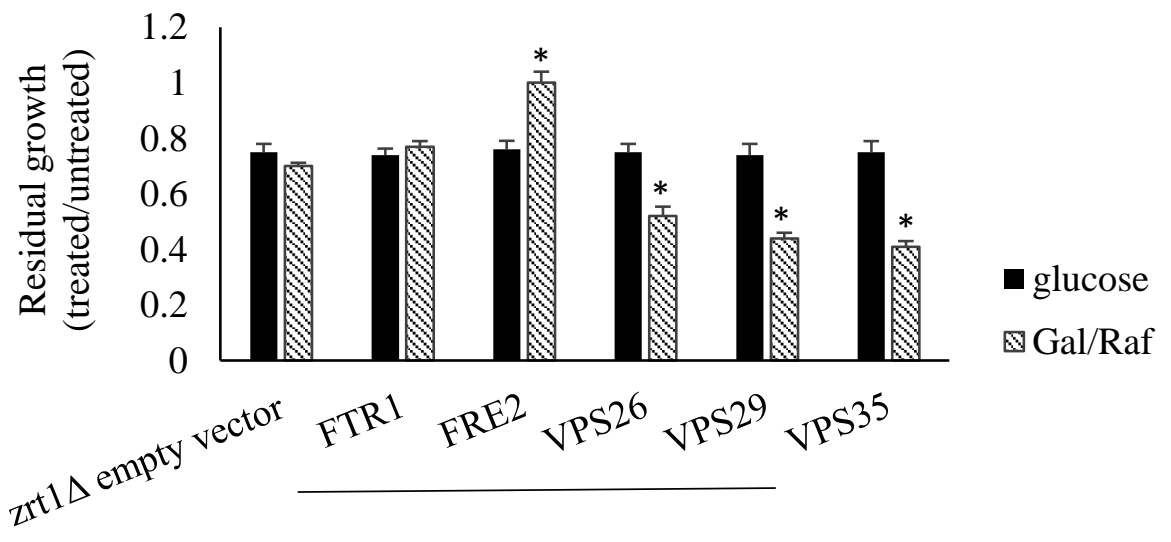

OE in $z r t 1 \Delta$

Figure 3-34. Overexpression of retromer and iron transporters in zrt14 strain. Effect of overexpression on growth was measured on glucose (control) and $2 \%$ Galactose/1\% Raffinose with/without $390 \mathrm{nM}$ EtOH-vescalagin. *, $p<0.05$, Student's $t$-test comparison with relative control. 


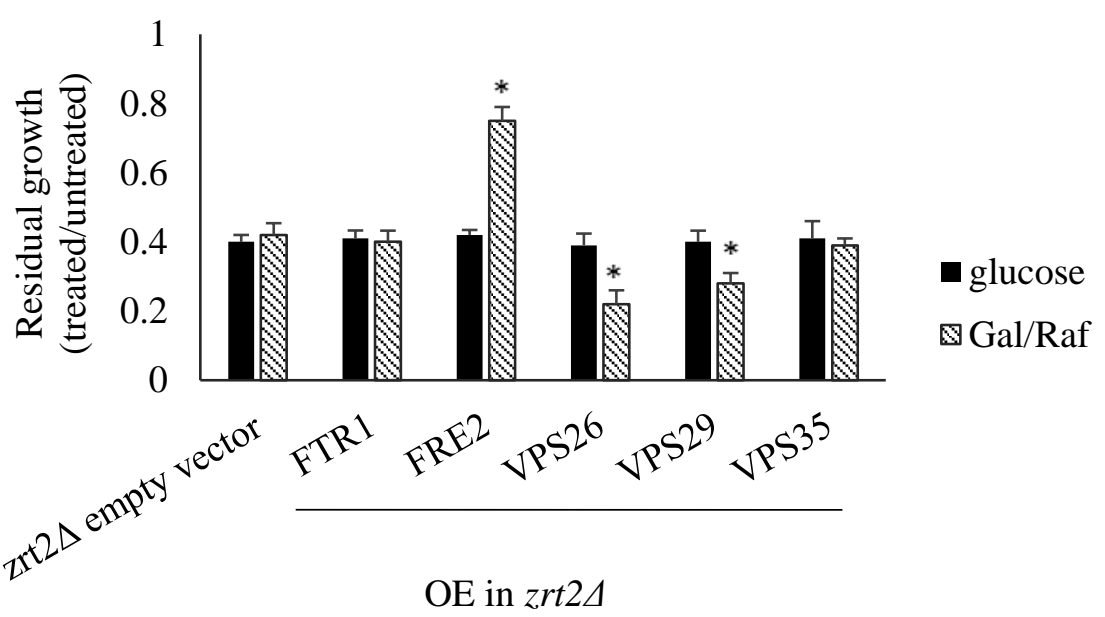

Figure 3-35. Overexpression of retromer and iron transporters in zrt2A strain. Effect of overexpression on growth was measured on glucose (control) and 2\% Galactose/1\% Raffinose with/without $390 \mathrm{nM}$ EtOH-vescalagin. *, $p<0.05$, Student's t-test comparison with relative control.

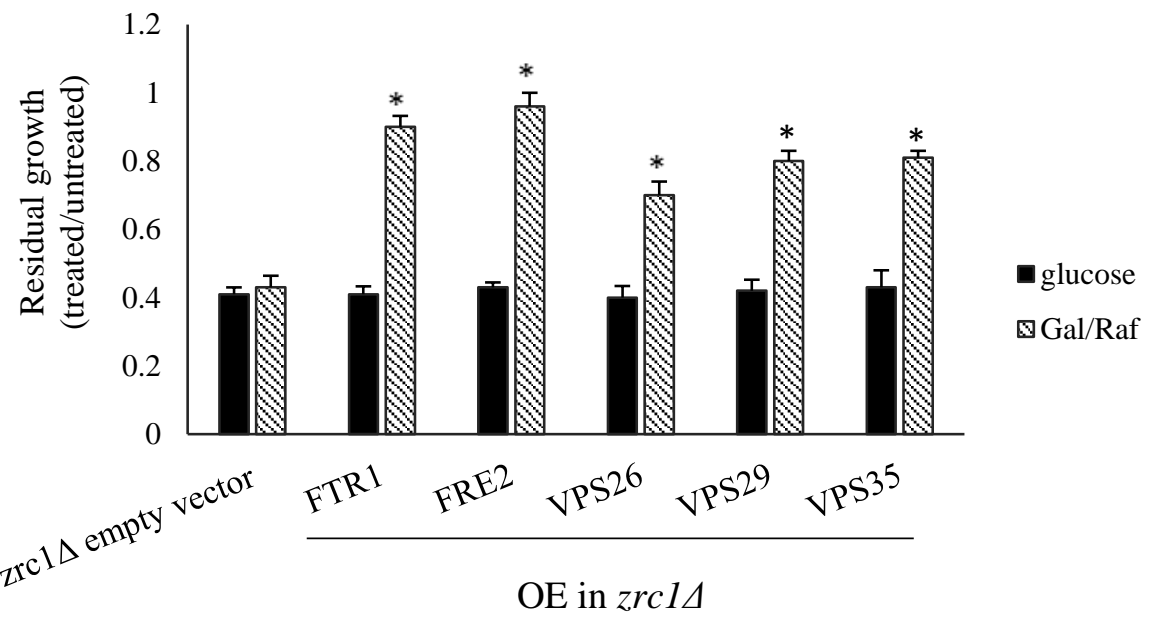

Figure 3-36. Overexpression of retromer and iron transporters recues zrc1 14 inhibition by EtOH-vescalagin. Effect of overexpression on growth was measured on glucose (control) and 2\% Galactose/1\% Raffinose with/without $390 \mathrm{nM}$ EtOH-vescalagin. *, $p<$ 0.05 , Student's t-test comparison with relative control. 


\subsubsection{Overexpression of iron and zinc transporters rescues sensitivity of retromer deletions to EtOH-vescalagin}

To investigate overexpression of iron and zinc transporter genes in retromer deletion strains, we transformed galactose-inducible overexpression plasmids of iron transporters (FTR1, FRE2) and zinc transporters (ZRT1, ZRT2, ZRC1) in retromer deletion strains (vps174, vps294). In the background of deletion of a gene from either the recognition sub-complex of the retromer (vps294)) or the packaging sub-complex of the retromer (vps 174), overexpression of FTR1 or FRE2 rescued sensitivity by $35-45 \%$ while no change was recorded with overexpression of zinc transporters (Figure 3-37 and Figure 3-38). These results further demonstrate that EtOH-vescalagin sensitivity due to retromer dysfuction can be rescued with increased iron uptake (via overexpression of FTR1 or FRE2).

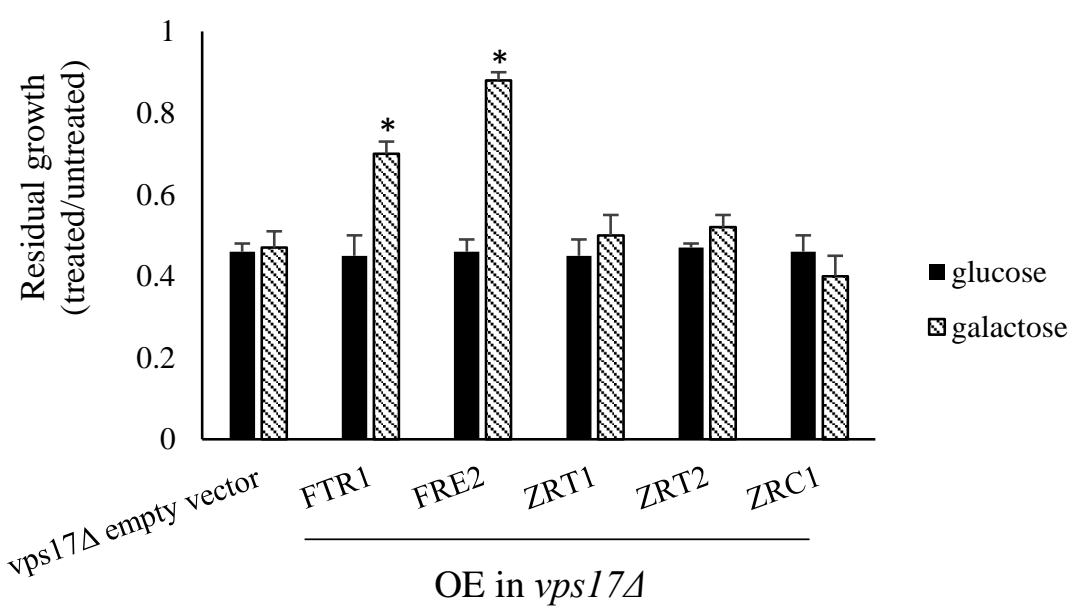

Figure 3-37. Overexpression of zinc and iron transporters in vps174 strain. Effect of overexpression on growth was measured on glucose (control) and 2\% Galactose/1\% Raffinose with/without $390 \mathrm{nM}$ EtOH-vescalagin. *, $p<0.05$, Student's $t$-test comparison with relative control. 


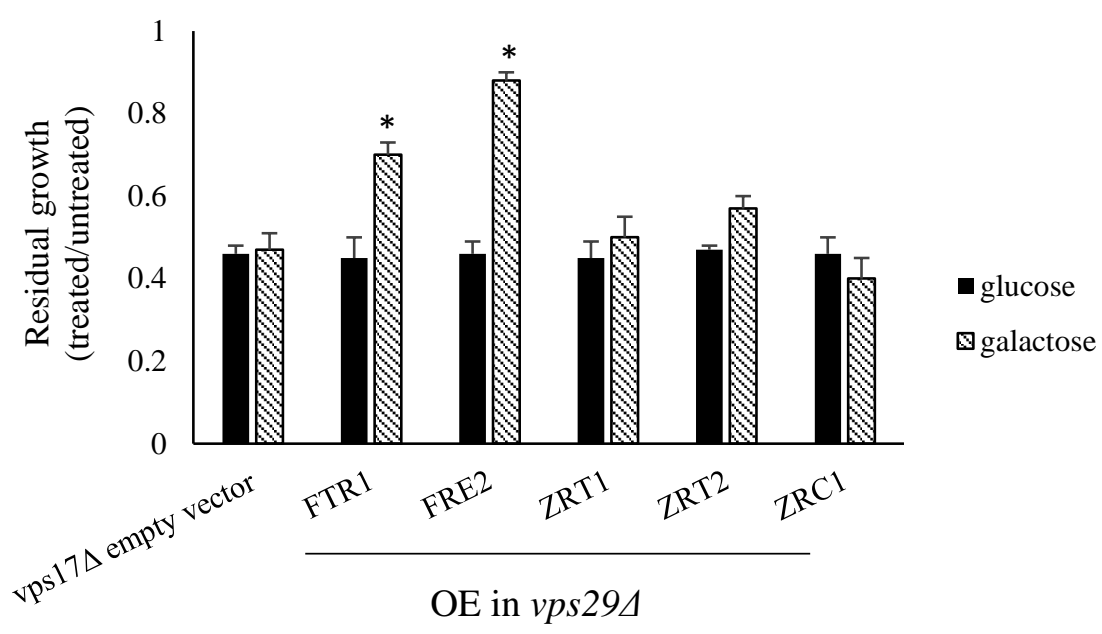

Figure 3-38. Overexpression of zinc and iron transporters in vps294 strain. Effect of overexpression on growth was measured on glucose (control) and $2 \%$ Galactose/1\% Raffinose with/without $390 \mathrm{nM}$ EtOH-vescalagin. *, $p<0.05$, Student's $t$-test comparison with relative control.

\subsubsection{EtOH-vescalagin disrupts mitochondrial function and respiration}

Mitochondrial function is dependent on iron and critical for cell respiration and energy metabolism (Yoon, et al. 2004). Based on our determination that EtOH-vescalagin chelates iron (Figure 3-21) and that several mitochondrial gene deletions (mrpl84, mrm24, iba574) were sensitive to EtOH-vescalagin (Figure 3-6), we hypothesized EtOH-vescalagin interrupts mitochondrial function. We thus tested two major mitochondrial iron transporters deletion strains ( $m r s 3 \Delta$ and $m r s 4 \Delta$ ) for sensitivity to EtOH-vescalagin. Treatment with EtOH-vescalagin significantly decreased the growth of $m r s 3 \Delta$ and $m r s 4 \Delta$ by 45 and 50\%, respectively, compared to the untreated control (Figure 3-39A). To examine a functional consequence of defective mitochondria, we measured an aspect of oxidative stress. We determined whether glutathione, a scavenger of reactive oxygen species, could ameliorate the growth defect caused by EtOHvescalagin. Using a concentration of $\mathrm{EtOH}$-vascalagin that dramatically reduced growth by $75 \%$, we determined that $1 \mathrm{mM}$ glutathione ameliorated this growth defect by $42 \%$, which was 
in the direction of normal, unaffected growth with the glutathione treatment alone (Figure 3.39B). These results indicate that an aspect of toxicity of EtOH-vescalagin includes oxidative stress, and this could perhaps be a consequence of the iron chelation mechanism.

A

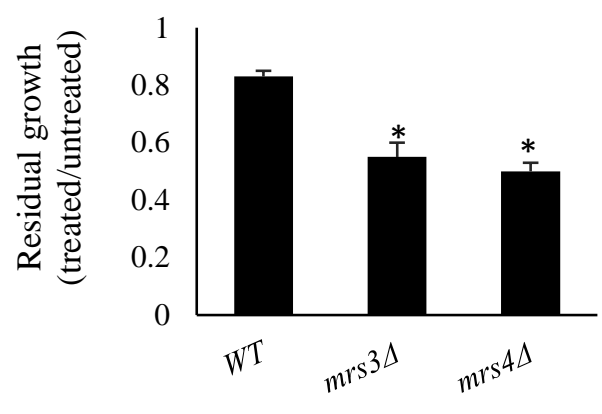

B

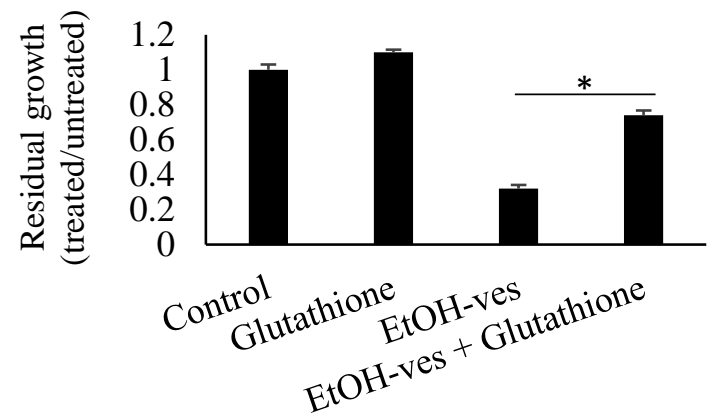

Figure 3-39. Mitochondrial function is regulated by EtOH-vescalagin. A) Growth inhibition of $m r s 3 \Delta$ and $m r s 4 \Delta$ by $305 \mathrm{nM}$ EtOH-vescalagin. *, $p<0.05$, Student's t-test comparison with WT. B) Growth inhibition of WT by $760 \mathrm{nM}$ EtOH-vescalagin with/without Glutathione. *, $p<0.05$, Student's t-test comparison with EtOH-vescalagin treatment in triplicate for each graph.

\subsection{DISCUSSION}

This chapter aimed to investigate the antifungal potential of EtOH-vescalagin and also the antifungal mechanism of action. Using unbiased genome-wide barcode sequencing analyses, we identified sensitive HET and HOM deletion strains to EtOH-vescalagin that resulted in determined iron chelation as a potential primary physical drug target with secondary targets of zinc homeostasis, retromer recycling and mitochondrial dysfunction.

We first proved that EtOH-vescalagin is antifungal in the nanomolar range against human pathogenic Candida species. This result was particularly interesting given that vescalagin did not inhibit growth of $C$. albicans and $S$. cerevisiae (Becker, et al. 2005). Our results would be consistent with the prediction that vescalagin in oak heartwood is one of the compounds that combats the growth of fungal pathogens in casks (Omar, et al. 2014) as well 
as several other ellagitannins (e.g., candelitannin and tellimagrandin II) that have exhibited antifungal activity (Lipińska, et al. 2014; Yamaguchi, et al. 2011). As EtOH-vescalagin is different in structure from vescalagin, we assume the antifungal activity of EtOH-vescalagin is a consequence of the ethanol adduct. Given clear demonstration that EtOH-vescalagin is antifungal, future side-by-side comparisons of antifungal activity can be conducted on vescalagin and EtOH-vescalagin.

We demonstrated the EtOH-vescalagin treatment results in iron deficiency in the cell based on sensitivity of iron transporter deletion strains, upregulation of iron transporters, rescued growth reduction with iron supplementation, and measurements of intracellular iron. Iron chelation can occur intracellularly as well as extracellularly (Philpott and Protchenko 2008; Puig, et al. 2005). Since we showed that EtOH-vescalagin chelated iron in a cell-free assay, we propose that EtOH-vescalagin chelates iron in the media thus inducing the cellular response to iron deficiency. The cellular response detected with $\mathrm{EtOH}$-vescalagin was consistent with other treatments that induced iron deficiency (Philpott and Protchenko 2008; Puig, et al. 2005). It is still plausible that EtOH-vescalagin chelates iron in the cell as well and this requires further investigation.

Polyphenols are secondary metabolites from plants containing phenolic backbone and aromatic rings which are prone to make a complex with metal ions. Iron chelation by plant polyphenols is a well-established mechanism underlying a range of bioactivities (Hider, et al. 2001) including condensed tannins (Karamać 2009). However, iron chelation has yet to be described for vescalagin or EtOH-vescalagin. The simultaneous consequence of iron chelation on zinc metabolism and retromer recycling would be interesting, as it was in this chapter, to investigate for other iron chelator ellagitannins. It is plausible that all ellagitannins (e.g., the compounds treated at the beginning of this chapter in Figure 3-2), like EtOH-vescalagin, 
increase intracellular zinc levels and disrupt retromer recycling. Most studies to date have not simultaneously examined iron chelation, zinc homeostasis and retromer recycling.

Zinquin staining, zinc quantification, and sensitivity of zinc transporter deletion strains indicate that EtOH-vescalagin increases intracellular levels of zinc. This mechanism is further supported with exacerbated growth inhibition with zinc supplementation. This mechanism requires alterations at the plasma membrane as well as the vacuole. At the plasma membrane, deletion of the high affinity zinc transporter ZRT1 suppresses the antifungal activity of EtOHvescalagin, presumably since the cell can tolerate increased intracellular levels of zinc with reduced uptake of zinc from the media. Our observation of reduced expression of Zrt1-GFP was also consistent with increased intracellular levels of zinc and other studies (Eide 2006). There are several reasons that these zinc alterations are a consequence of iron chelation. First, zinc and iron compete as substrates for ferrochelatase in yeast (Camadro and Labbe 1988). Second, FET4 is regulated by an iron transcription factor (AFT1) as well as a zinc transcription factor (ZAP1) (Rutherford and Bird 2004). Third, iron deficiency-mediated oxidative stress can induce zinc uptake (Santos, et al. 2003). However, there was not complete concordance in our results when we compared the iron chelator BPS and EtOH-vescalagin. Notably, BPS treatment increased expression of Zrt1-GFP suggesting that iron chelation by BPS simultaneously decreases both iron and zinc in the cell, which would be inconsistent with our observation that EtOH-vescalagin decreased intracellular iron and increased intracellular zinc.

Our result also showed that retromer function is disrupted by EtOH-vescalagin and this is likely related to alterations in iron and zinc. Regarding iron, the retromer proteins Vps10GFP, Vps17-GFP and Vps26-GFP responded to EtOH-vescalagin and mislocalized from the endosome to the vacuolar membrane; this was possibly a response to iron chelation since the BPS treatment replicated this phenotype. Interestingly, this mislocalization of Vps10-GFP was present in all strains except $z r t 1 \Delta$, suggesting that the retromer complex can function normally 
when zinc levels are not increased. We also identified three strong connections between retromer, iron and zinc. First, EtOH-vescalagin sensitivity was reduced in retromer deletion strains with iron supplementation or overexpression of iron transporters at the plasma membrane. Second, EtOH-vescalagin sensitivity was exacerbated in retromer deletion strains with zinc supplementation. Third, EtOH-vescalagin sensitivity was exacerbated with overexpression of retromer subunits in zrtlA (low intracellular zinc), while sensitivity was reduced with overexpression of retromer subunits in $\operatorname{rrcl\Delta }$ (high intracellular zinc). Overall, our results suggest iron, zinc and retromer need to be in balance with each other to function properly. Consistent with our results would be the reports that VPS10- and VPS29-dependent phosphatase activity requires zinc to function (Damen, et al. 2006).

A critical aspect to zinc homeostasis is the ability of the cell to store excess zinc in the vacuole via the ZRC1 and COT1 transporters at the vacuolar membrane (MacDiarmid, et al. 2000). We demonstrated that the $z r c 1 \Delta \cot 1 \Delta$ double deletion was the most sensitive strain to EtOH-vescalagin. As other strains with functional ZRC1 and COT1 are also sensitive to excess zinc, it is possible that in those strains the retromer defect by iron chelation causes the vacuolar defects leading to zinc leakage from vacuole and zinc toxicity. This was shown earlier that in GARP deletion strains such as vps534 (golgi associated retrograde protein) that are involved in endosome to golgi transport (same as retromer complex), ZAP1 transcription is significantly decreased including ZRT1 (Fröhlich, et al. 2015). This study suggested that vacuolar dysfunction in vps534 causes increased zinc release from vacuole and zinc toxicity. This phenomenon may be part of the EtOH-vescalagin mechanism as well though more clarification needs to be done (Fröhlich, et al. 2015).

As mitochondria require iron for several enzymes (Fe-S cluster, Heme, electron transport chain) (Yoon, et al. 2004), the iron chelation mechanism of EtOH-vescalagin may have resulted in mitochondrial dysfunction and low respiration. This can also cause an 
increasing ROS level which is toxic to the cell and decreases the life span as well. This result is also consistent with previous studies stating iron chelation causes respiration damage and decreased life span (Zuin, et al. 2008). Based on sensitivity of DNA repair gene deletion strains and the reports that DNA replication and repair is dependent on cytosolic and mitochondrial Fe-S clusters (Rouault 2012), it is also plausible that these processes are also affected by the iron chelation mechanism of EtOH-vescalagin.

In conclusion, the HIP-HOP analysis was fundamental to proposing a working model for the mechanism of action of EtOH-vescalagin (Figure 3-40). EtOH-vescalagin binds to iron in the media, making it unavailable to the cell and causing disrupted retromer recycling. Zinc toxicity then occurs as a result of retromer disruption, vacuolar dysfunction and zinc leakage from the vacuole. Both BPS and EtOH-vescalagin cause mitochondrial damage indirectly, which leads to increased ROS levels and respiration defects (Figure 3-40). Our result showed EtOH-vescalagin is chelating iron to the same extent as BPS and impacting mostly the same pathways; however, there were some differences between EtOH-vescalagin and BPS effected phenotypes that suggest functional differences such as in zinc homeostasis (BPS treatment did not result in increased intracellular zinc while EtOH-vescalagin did). As EtOH-vescalagin showed potent antifungal activity in contrast to vescalagin, we assume the effects are a consequence of the ethanol adduct in this structure. 
A) control

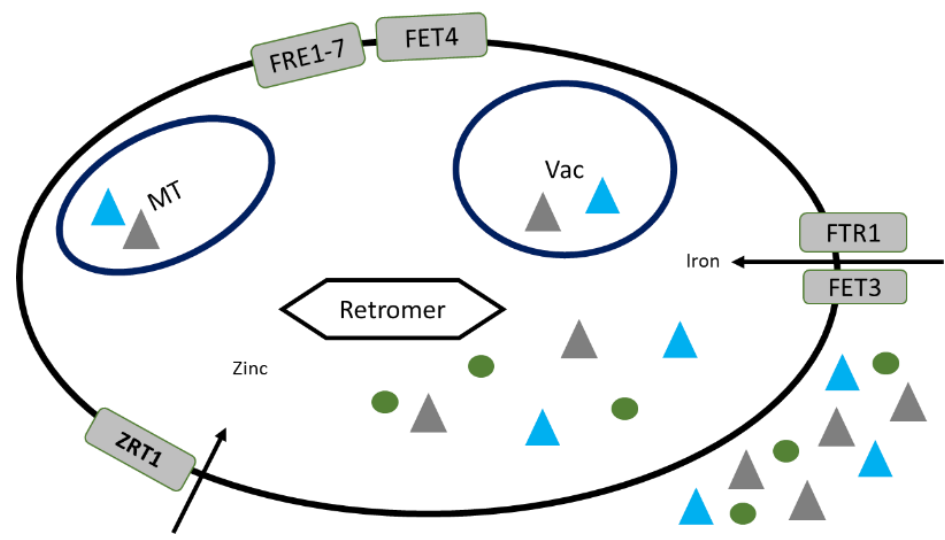

B) EtOH-vescalagin

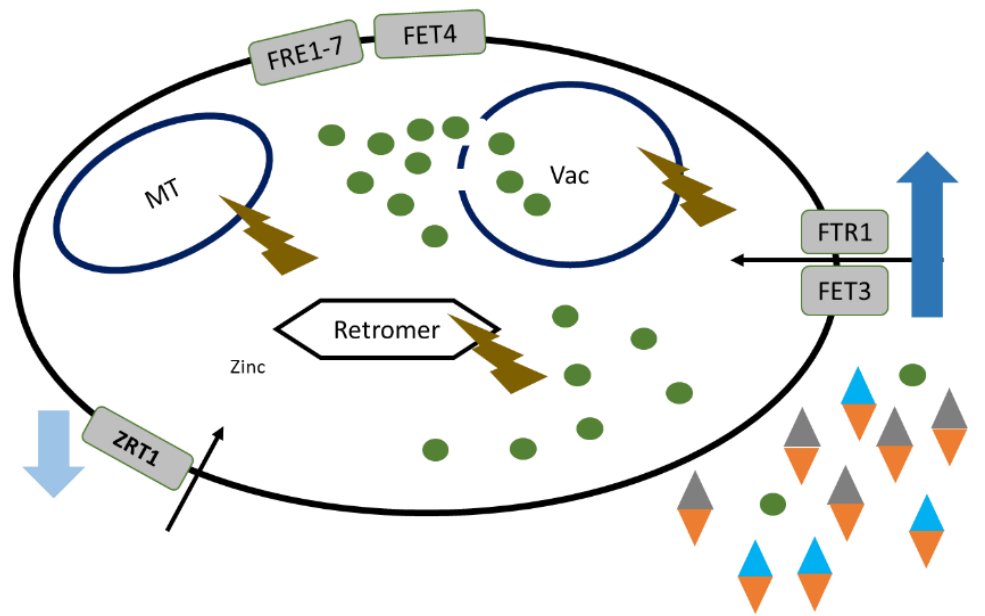

EtOH-ves

Fe II

Fe I

zinc

disruption
(EtOH-ves)-iron I

(EtOH-ves)-iron II

downregulation

upregulation 
C) MOA of EtOH-

vescalagin in functions

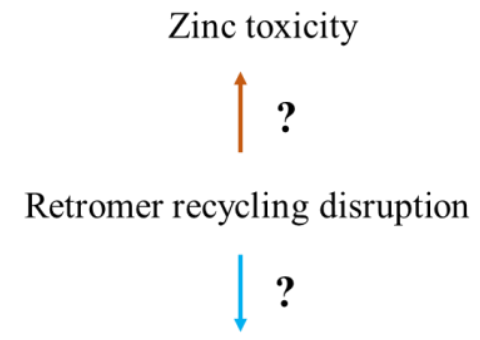

Zinc deficiency
BPS EtOH-ves

$\downarrow$

Extracellular Iron chelation

I

Low intracellular iron<smiles>C=[13CH]</smiles>

Iron-S cluster/Heme disruption

I

Mitochondrial disfunction

Figure 3-40 Model of EtOH-vescalagin mechanism of action in S. cerevisiae. A) The normal untreated cell with normal iron uptake rate and cell is functioning properly. B) The EtOH-ves (vescalagin) treated cell causes iron chelation extracellularly and cell functions are disrupted while zinc is increasing intracellularly. Ret: Retromer. Vac: vacuole. MT: mitochondria. C) MOA of EtOHvescalagin with probable inhibited functions. 



\section{Chapter 4 Elucidating the Antifungal Mechanism of Action of Estragole}

\subsection{INTRODUCTION}

Approximately 1.2 billion people suffer annually from fungal infections around the world (Denning and Bromley 2015). Therefore, effective antifungal drugs are critical to treating these infections. As these infections are caused by taxonomically diverse fungi, the currently approved treatments target processes conserved across fungal species. There are five groups of antifungal drugs based on their mechanism (Campoy and Adrio 2017) (Figure 4.1). The first group targets ergosterol homeostasis, the fungal equivalent of cholesterol that is a major component of plasma membrane in fungi. The second group inhibits the synthesis of chitin and peptidoglycan, major components in the fungal cell wall. The third group inhibits the synthesis of sphingolipids, a class of lipids that are critical for the lipid bilayer in fungal plasma membranes. The fourth and fifth groups inhibit nucleic acid synthesis and protein synthesis, respectively, which are critical processes integral to converting DNA to proteins. As there is heavy usage of these antifungal drugs, fungal pathogens continue to develop resistance against these drugs (Eliopoulos, et al. 2002). It is critical to elucidate the mechanism of action of all drugs exhibiting antifungal activity particularly whether the new drug targets the same mechanism as the currently available drugs. 


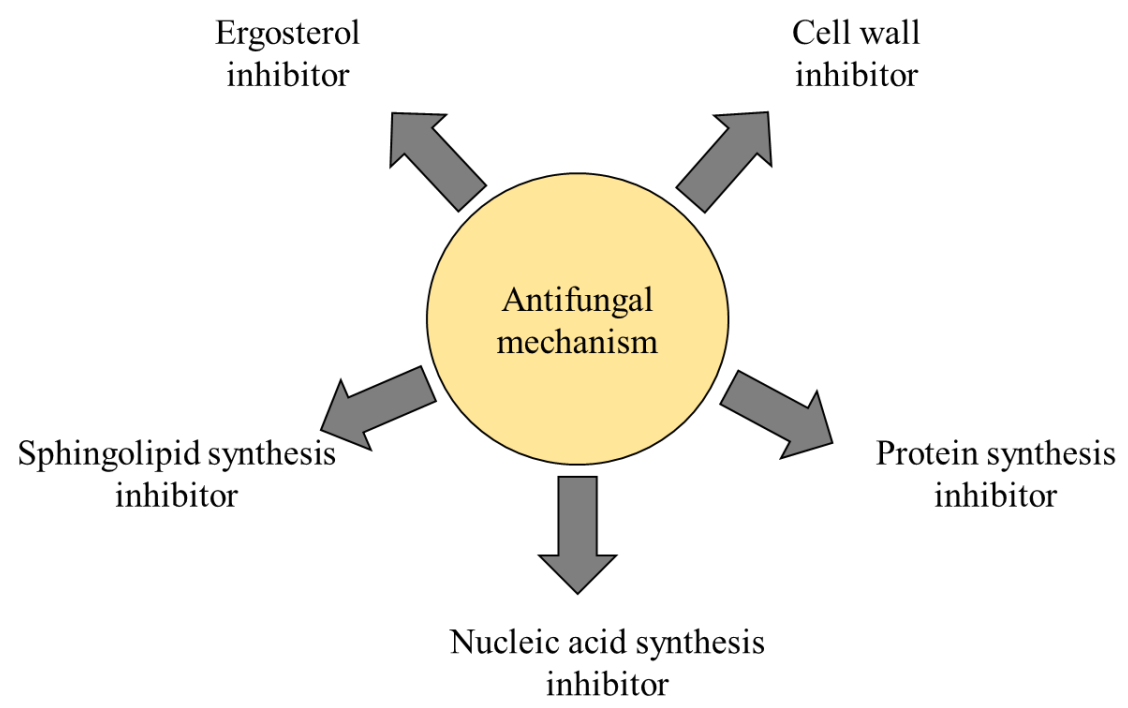

Figure 4-1. Established antifungal targets.

Estragole (also known as 4-allylanisole, p-allylanisole and methyl chavicol) is a phenylpropene that has previously been identified in feijoa (Saj, et al. 2008). Specifically, it is an organic compound composed of a benzene ring substituted with a methoxy group and a propenyl group (Figure 4.2). Estragole is actually not specific to feijoa as it has also been identified in other plants such as fennel, tarragon, pine oil and feijoa (Ashurst 2012). Estragole has been shown to exhibit antifungal activity via growth inhibition of pathogenic Blastoschizomyces capitatus, Candida albicans and Trichophyton species (Shin 2004; Shin and Kang 2003; Shin and Pyun 2004). However, the antifungal mechanism of estragole is not known. Based on the report that estragole has a synergistic antifungal effect with ketoconazole (Shin and Kang 2003), we hypothesize that estragole is antifungal via a mechanism distinct from the antifungal mechanism of ketoconazole. Ketoconazole is toxic to fungal cells by inhibiting ERG11, a key enzyme in the biosynthesis of ergosterol, a major component of the fungal plasma membrane (Heimark, et al. 2002). 


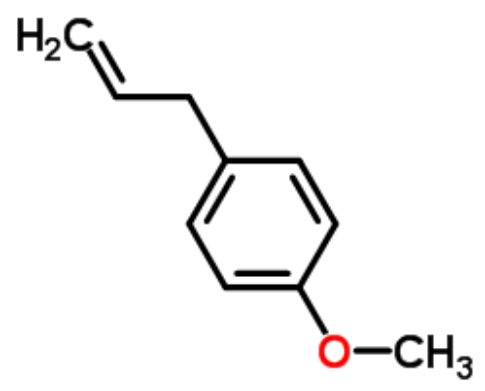

Figure 4-2. Estragole structure. Drawn using ChemSpider.

Given that chemical genetics is an established method to determine mechanism of action of compounds (Parsons, et al. 2006), here we explored the antifungal mechanism of action of estragole using chemical genetics. We used chemical genetics, mass spectrometry, and fluorescent microscopy to determine that ergosterol, the target of ketoconazole, is not the target of estragole. We also used chemical genetics to determine that the target of estragole is not other known antifungal targets including sphingolipid synthesis, cell wall synthesis, nucleic acid synthesis, and protein synthesis. Genome-wide barcode sequencing was then conducted to obtain an unbiased insight into the antifungal mechanism of action.

\subsection{METHODS}

\subsubsection{Antifungal activity}

Antifungal activity of estragole (provided from Sigma) was measured in biological and technical triplicate as previously described (Ruggles, et al. 2014) (section 2.3.4) against a laboratory strain of Saccharomyces cerevisiae (the wild-type strain BY4741) as well as human

pathogenic isolates of Candida albicans (ATCC 10231), C. glabrata (ATCC 90030), C. parapsilosis (ATCC 90018), and C. tropicalis (ATCC 13803). The growth of $5 \times 10^{5}$ cells $/ \mathrm{mL}$ in SC media (HEPES with PH of 8 was added to the SC media to a final concentration of 25 
$\mathrm{mM}$, and hereafter is referred to as SC) was measured via an absorbance reading with an Envision plate reader (Perkin Elmer) at $600 \mathrm{~nm}$ after 14 hours (a time point in which untreated cells are at mid-log), and residual growth was calculated based on the ratio of treated and untreated cells.

\subsubsection{Hypersensitivity assay}

Heterozygous diploid mutant strains sensitive to existing known antifungal drugs were grown in the presence and absence of estragole using the antifungal assay described in section 4.2.1.

\subsubsection{Fluorescent microscopy analysis of ergosterol}

Abundance and localization of free ergosterol were monitored using fluorescent microscopy of cells stained with filipin as previously described (Van Leeuwen, et al. 2008). Cells were grown for $14 \mathrm{~h}$ in $2 \mathrm{~mL} \mathrm{SC}$ at $30^{\circ} \mathrm{C}$ with and without estragole. Then $1 \mathrm{OD}$ unit (1 $\mathrm{ml}$ of OD of 1) was centrifuged at 13,000 rpm for $5 \mathrm{~min}$ and cell pellets were resuspended in $500 \mu \mathrm{L}$ SC, to which $100 \mu \mathrm{g} / \mathrm{mL}$ filipin was added and cells were immediately viewed with 100X magnification using the DAPI filter on a fluorescent microscope (Olympus BX63).

\subsubsection{Mass spectrometry}

Ergosterol and ergosterol precursors were quantified using GC-MS as previously described (Arthington-Skaggs, et al. 1996). In brief, cells were incubated overnight at $30^{\circ} \mathrm{C}$, total lipids were extracted from 5 OD units ( $5 \mathrm{ml}$ of OD of 1 ) as previously described (Folch, et al. 1957), and dried lipids were derivitized and silylated as previously described (Moser 2011). Then $1 \mu \mathrm{L}$ of the final volume was injected in the Shimadzu QP2010-Plus GC-MS (Shimadzu, Kyoto, Japan). Ergosterol and the precursor lanosterol were identified by aligning 
chromatograms of standards and samples with additional comparison with the NIST11 database. Sterols in samples were then quantified relative to a standard curve of the standards.

\subsubsection{Genome-wide barcode sequencing analysis}

Sensitivity of homozygous and heterozygous mutations to estragole was evaluated using barcode sequencing as previously described (Robinson, et al. 2014) and in Chapter 3 (section 3.2.4). In brief, pools of the diploid heterozygous deletion library or pools of the diploid homozygous deletion library were grown in the presence and absence of estragole. Barcodes flanking each gene deletion were quantified using an Illumina HiSeq 2000 platform, and candidate sensitive strains were independently validated. Candidate strains grown overnight in SC media in 96 -well plates at $30^{\circ} \mathrm{C}$ were inoculated into 96 -well plates containing SC with and without estragole, incubated at $30^{\circ} \mathrm{C}$ to mid-log growth ( $\mathrm{OD}_{600}$ of 0.4 in untreated plate), and growth was measured using the Envision plate reader (Perkin Elmer). Sensitive genes were analysed for function using Gene Ontology (Ashburner, et al. 2000) and for network interactions using GENEMANIA (Warde-Farley, et al. 2010).

\subsubsection{Mitochondria analysis}

Mitochondria were visualised using fluorescent microscopy as previously described (Marobbio, et al. 2003). In brief, mid-log cells grown at $30^{\circ} \mathrm{C}$ were stained with $100 \mathrm{nM}$ Mitotracker Red CMXRos (ThermoFisher), incubated at $30^{\circ} \mathrm{C}$ for $30 \mathrm{~min}$ and immediately viewed with 100X magnification using the Texas Red filter on a fluorescent microscope (Olympus BX63). 


\subsection{RESULTS}

\subsubsection{Estragole is bioactive and antifungal}

To initially investigate the antifungal activity of estragole, we quantified the efficacy of estragole to inhibit the growth of a wild-type laboratory strain of S. cerevisiae (BY4741). We compared growth of control cells and cells treated with various concentrations of estragole (50-400 $\mu \mathrm{M})$. Significant growth inhibition was not achieved at $50 \mu \mathrm{M}$ estragole, while increasing concentrations at 100, 200 and $400 \mu \mathrm{M}$ estragole significantly inhibited growth by 25, 56 and 75\%, respectively, compared to untreated cells (Figure 4.3). These results indicate that estragole inhibits the growth of $S$. cerevisiae, a result consistent with previous reports that estragole exhibits antifungal activity against Trychophyton species, Candida albicans, and Blastoschizomyces capitatus (Shin 2004; Shin and Pyun 2004).

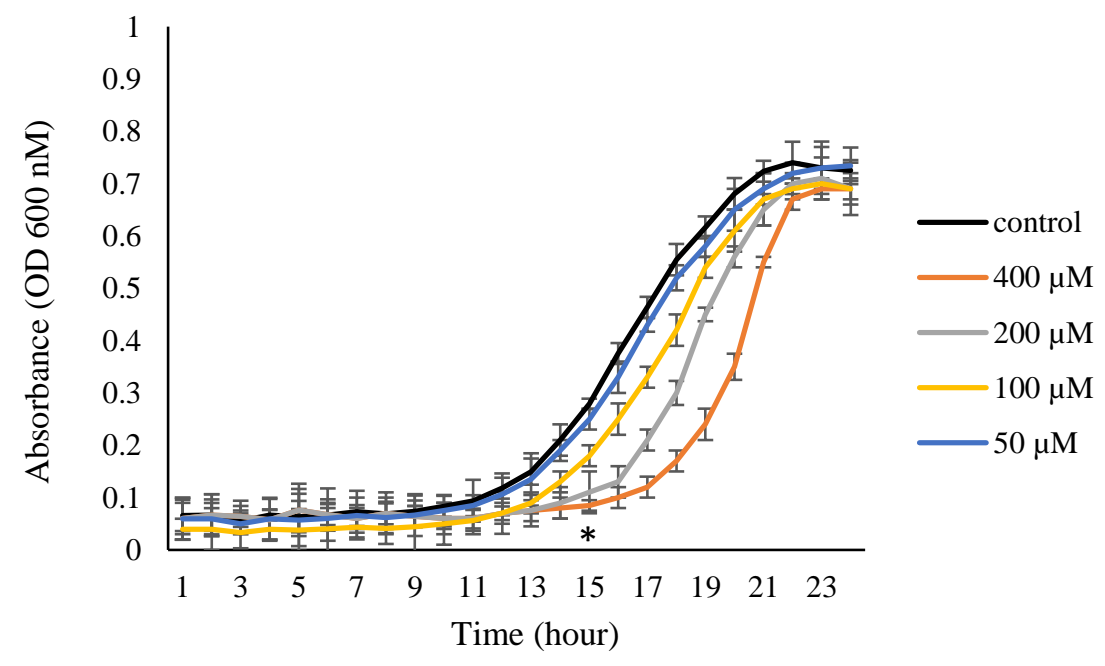

Figure 4-3. Estragole dose response in WT BY4741. Cells were grown in SC media and treatments compared to the vehicle control (DMSO) while absorbance measured every hour at $\mathrm{OD}_{600} *^{*}, p<0.05$, student's $t$-test comparison of control and each $100 \mu \mathrm{M}, 200 \mu \mathrm{M}, 400 \mu \mathrm{M}$.

To determine if estragole inhibits growth of pathogenic fungal species, we measured the growth of human pathogenic Candida species (C. albicans, C. glabrata, C. parapsiliosis, 
C. tropicalis) in the presence of estragole. We calculated an $\mathrm{IC}_{50}$ value based on growth inhibition at 50,100, 200 and $400 \mu \mathrm{M}$ estragole compared to untreated cells. The half-maximal inhibitory concentration ( $\mathrm{IC}_{50}$ ) of estragole was $215,186,178$ and $233 \mu \mathrm{M}$ against $C$. albicans, C. glabrata, C. parapsiliosis and C. tropicalis, respectively (Table 4.1). These results are consistent with an $\mathrm{IC}_{50}$ of $151 \mu \mathrm{M}$ against $S$. cerevisiae, suggesting that the antifungal activity of estragole is similar against laboratory strains as well as pathogenic strains.

Table 4-1. Half maximal inhibitory concentration $\left(\mathrm{IC}_{50}\right)$ of estragole in pathogenic Candida species.

\begin{tabular}{cccc}
\multicolumn{4}{c}{$\mathrm{IC}_{50}(\mu \mathrm{M})$ of estragole } \\
\hline C. albicans & C. glabrata & C. parapsilosis & C. tropicalis \\
\hline $215.1 \pm 0.5$ & $186.4 \pm 0.3$ & $177.9 \pm 0.6$ & $233.3 \pm 0.4$ \\
\hline
\end{tabular}

\subsubsection{The ketoconazole drug target ERG11 is not the target of estragole}

Determining the target of any drug is critical to understanding the molecular mechanism of action of that drug (Marton, et al. 1998). Reducing the gene copy number in diploid heterozygous deletion strains confers these strains hypersensitive to the drug that is targeting the product of that gene (Giaever, et al. 1999). We next sought to determine if estragole has the same ERG11 target as ketoconazole, a commonly prescribed antifungal drug that inhibits ergosterol biosynthesis and kills fungal cells by depleting ergosterol levels in the fungal plasma membrane (Figure 4.4A). Growth of WT and erg11 $/$ ERG11 was compared in $20 \mu \mathrm{M}$ ketoconazole and $90 \mu \mathrm{M}$ estragole, concentrations that inhibit the growth of WT by $20 \%$ compared to untreated cells. As previously observed (Lee, et al. 2014), erg114/ERG11 was significantly sensitive to ketoconazole with $71 \%$ inhibition compared to untreated cells $(P=$ 0.04), and this sensitivity was not observed in WT cells (Figure 4.4B). In contrast, both WT and $\operatorname{erg} 11 \Delta / E R G 11$ cells were similarly sensitive to estragole albeit only by $\sim 20 \%(P=0.03$ 
and $P=0.04$, respectively) compared to untreated cells (Figure 4.4B). These results suggest that ERG11 is not the target of estragole.

A

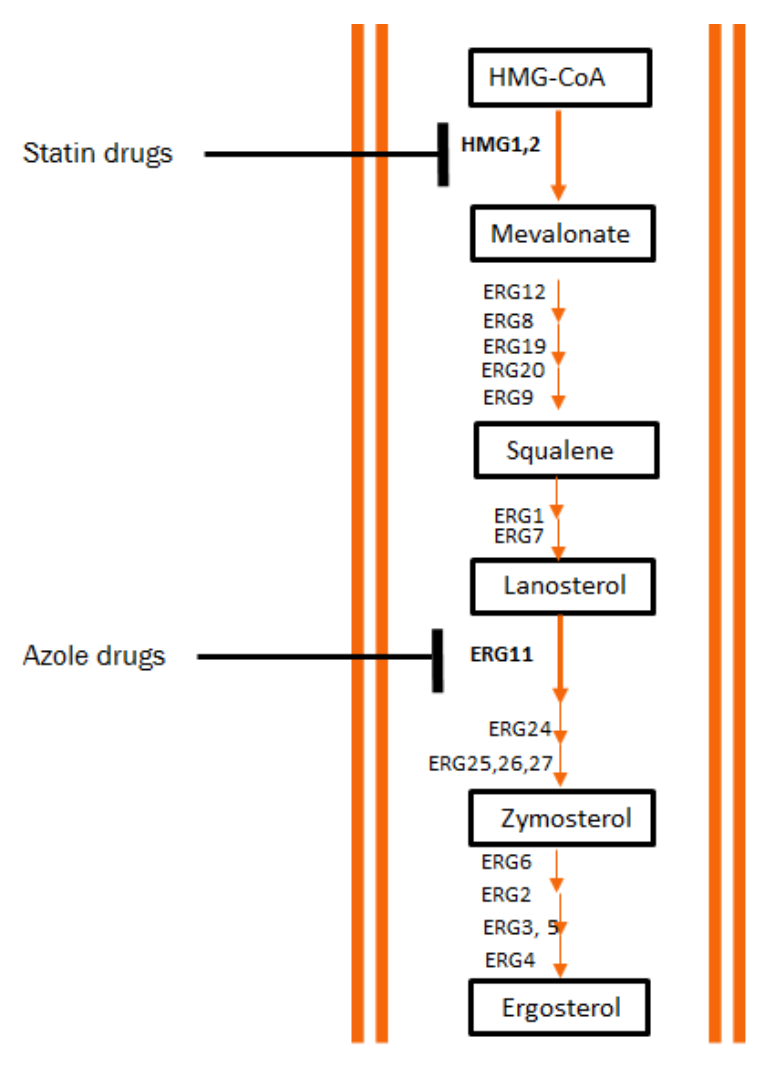

B

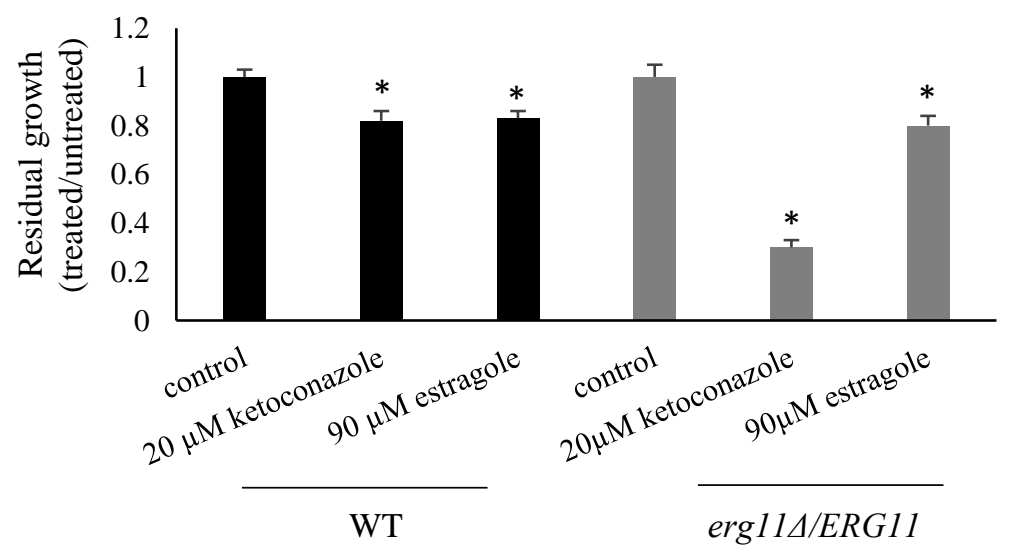

Figure 4-4. ERG11 is not the primary target of estragole. A) Ergosterol biosynthesis pathway. B) Cells were treated by estragole and ketoconazole compared to relative WT control. *, $p<0.05$, Student's $t$-test comparison with relative control. 


\subsubsection{Ergosterol biosynthetic genes are not the target of estragole}

Ergosterol homeostasis is a common antifungal target, whether by targeting ergosterol biosynthesis (e.g., atorvastatin, ketoconazole) or ergosterol itself (e.g., Nystatin). To examine if ergosterol biosynthetic genes other than ERG11 are the target of estragole, we measured the growth of ten heterozygous diploid strains mutated in various steps of ergosterol biosynthesis

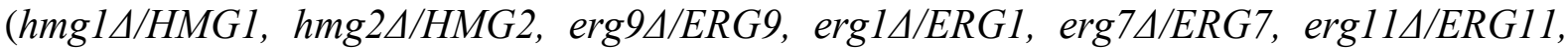
erg6/ERG6, erg2 $/ E R G 2$, erg24 /ERG24, erg44/ERG4) (Figure 4-5). All strains were grown in the presence and absence of $90 \mu \mathrm{M}$ estragole ( $\sim \mathrm{IC}_{20}$ in WT cells). If any of these genes were the target of estragole, the heterozygous deletion mutants will be hypersensitive to estragole. Four genes in various sections of the ergosterol pathway were significantly sensitive

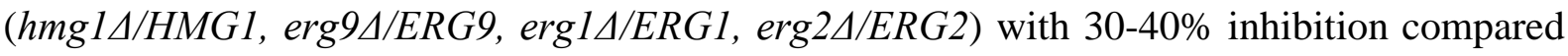
to untreated cells (Figure 4.5), though not to the extent that is observed for established targets of drugs (e.g., > 60\% inhibition of erg114/ERG11 conferred with ketoconazole treatment). These results suggest that ergosterol biosynthesis is involved in the antifungal activity of estragole but it is not the primary target of estragole.

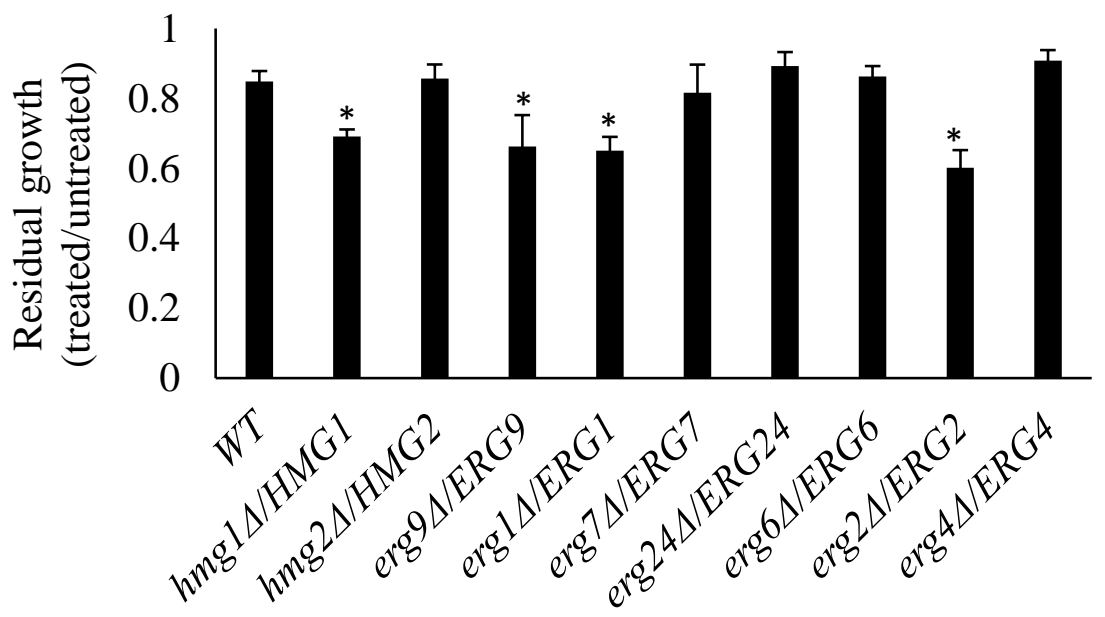

Figure 4-5. Genes in ergosterol pathway are not primary target of estragole. erg $1 \triangle / E R G 1$, $\operatorname{erg} 2 \Delta / E R G 2, \operatorname{erg} 9 \Delta / E R G 9, h m g 1 \Delta / H M G 1$ are sensitive to estragole though not hypersensitive while the rest of deletion strains are not sensitive. Values are triplicate and growth was measured at 14 hours at $\mathrm{OD}_{600}{ }^{*}, p<0.05$, Student's $t$-test comparison with WT. 


\subsubsection{Ergosterol homeostasis is not the target of estragole}

To confirm that ergosterol is not the target of estragole, we measured the localization and abundance of ergosterol using two methods. First, fluorescent microscopy was used to visualize ergosterol using filipin, a fluorescent stain of ergosterol (Van Leeuwen, et al. 2008). Consistent with previous results (Van Leeuwen, et al. 2008), we observed ergosterol localization in the plasma membrane of untreated WT cells and this localization in the plasma membrane was not altered with estragole treatment (Figure 4.6). These results indicate that estragole did not disrupt the intracellular trafficking of ergosterol.

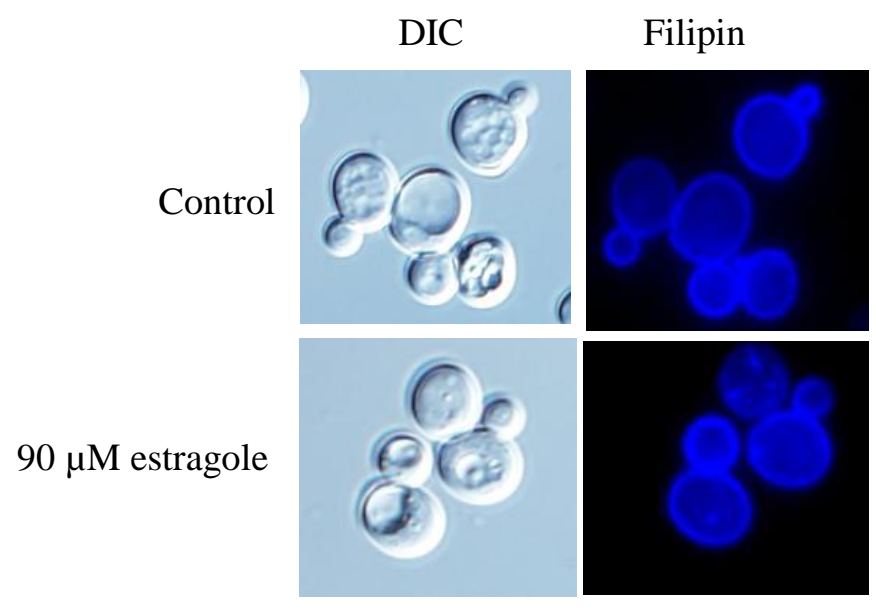

Figure 4-6. Estragole does not mislocalize or change abundance of ergosterol in plasma membrane. Filipin staining with/without estragole in WT. Cells were visualized under the Olympus fluorescent microscope with DAPI filter.

Second, we used GC-MS to quantify lanosterol and ergosterol. Quantification of these sterols is a hallmark phenotype for cells treated with azole-type antifungals (e.g., cells treated with ERG11-targeting fluconazole exhibit increased lanosterol and reduced ergosterol since ERG11 catalyzes the conversion of lanosterol to ergosterol) (Figure 4.4 A ) (Parks and Casey 1995). As previously demonstrated (Arthington-Skaggs, et al. 1996), we quantified a significant $83 \%$ reduction in ergosterol and a significant $82 \%$ increase in lanosterol in fluconazole-treated 
cells compared to control (Figure 4.7). In contrast, there were no significant differences between estragole-treated cells and untreated control cells (Figure 4.7). These results are consistent with the filipin results whereby both results indicate that ergosterol synthesis is not targeted by estragole.

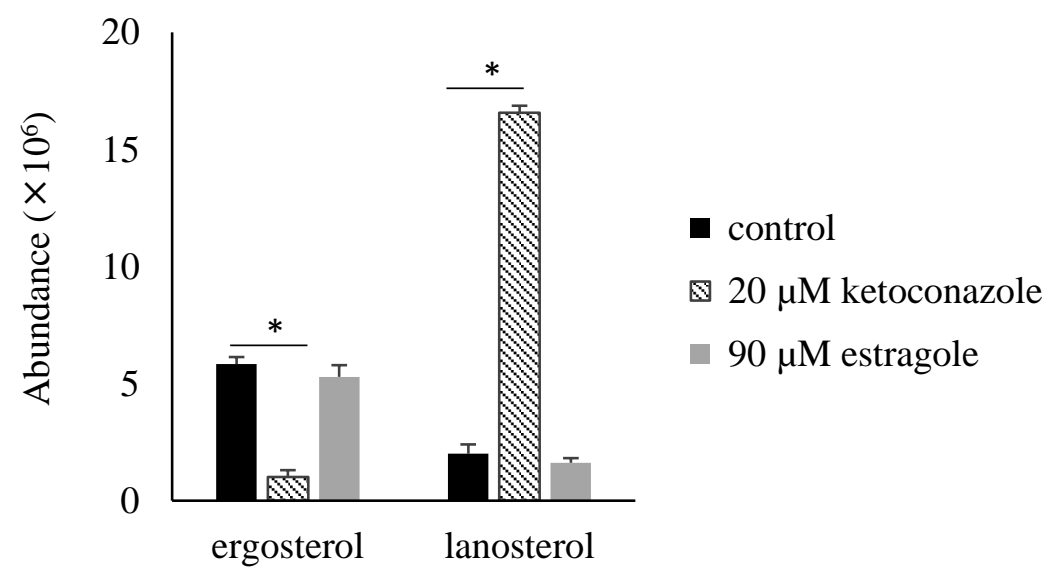

Figure 4-7. GC-MS quantification of sterols in estragole- and ketoconazole-treated WT cells.

$*, p<0.05$, Student's $t$-test comparison with relative control.

\subsubsection{Estragole does not target ergosterol in the plasma membrane}

Nystatin and amphotericin B are representatives of the polyene group of antifungals that target and bind to ergosterol itself resulting in increased permeability and disrupted membrane stability of the fungal lipid membrane (Marty and Finkelstein 1975). As these drugs bind directly to ergosterol, there is no gene target but there are deletion strains sensitive to these drugs. For example, the pop1 $1 / P O P 1$ strain is sensitive to both nystatin and amphotericin B (Lee, et al. 2014). To determine if the mechanism of estragole is similar to these drugs, we measured growth of pop1 $1 / P O P 1$ in $3 \mu \mathrm{M}$ nystatin and $90 \mu \mathrm{M}$ estragole, concentrations that inhibit the growth of WT by $20 \%$. As previously observed (Lee, et al. 2014), pop1A/POP1 was significantly sensitive to nystatin with $63 \%$ inhibition $(P=0.03)$ compared to untreated cells, and this sensitivity was not observed in WT cells (Figure 4.8). In contrast, both WT and 
pop1 $1 / P O P 1$ cells were not largely sensitive to estragole albeit only by $\sim 20 \%(P=0.02$ and $P$ $=0.04$, respectively) compared to untreated cells (Figure 4.8). These results further demonstrate that ergosterol is not the target of estragole.

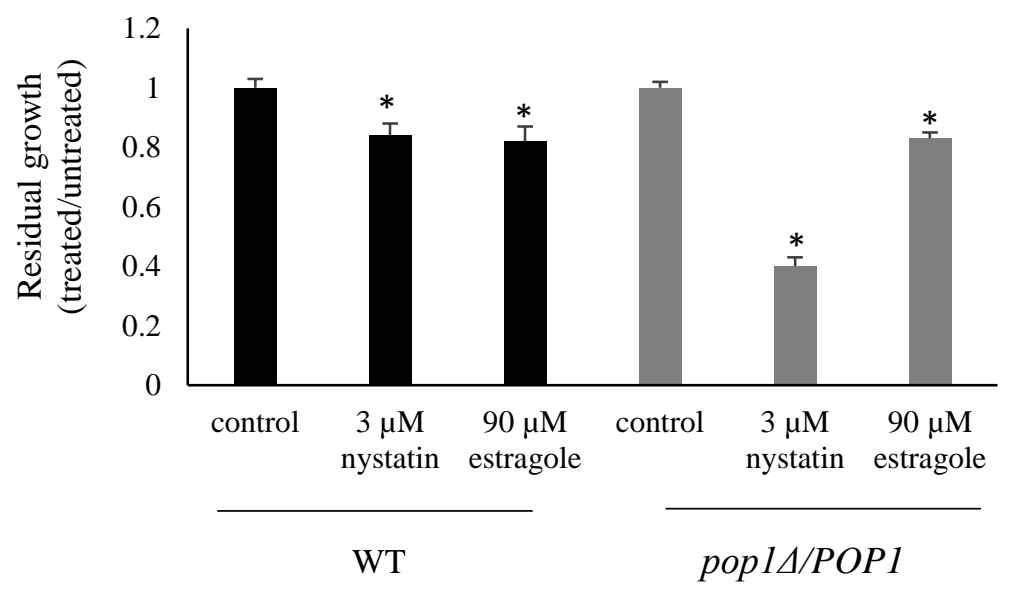

Figure 4-8. Estragole mechanism is distinct from nystatin. WT treatments compared to the relative WT control and pop1 $1 /$ POP1 treatments compared to the relative control. Values are residual growth at 14 hours in triplicate in SC media measured at $\mathrm{OD}_{600} .{ }^{*}, p<0.05$, Student's t-test comparison with relative control.

\subsubsection{The echinocandin target FKS1 is not the target of estragole}

Echinocandins are a class of antifungals targeting $\beta$-(1,3)-glucan synthesis in the fungal cell wall (Denning 2003). To determine if estragole has the same FKS1 target as caspofungin, an exemplar drug of a commonly prescribed echinocandin, growth of WT and $f k s 1 \Delta / F K S 1$ was compared in $20 \mu \mathrm{M}$ ketoconazole and $12 \mu \mathrm{M}$ caspofungin, concentrations that inhibit the growth of WT by $20 \%$ compared to untreated cells. As previously observed (Lesage, et al. 2004), $f k s 1 \Delta / F K S 1$ was significantly sensitive to caspofungin with $76 \%$ inhibition $(P=$ 0.03) compared to untreated cells, and this sensitivity was not observed in WT cells (Figure 4.9). In contrast, both WT and $f k s 1 \Delta / F K S 1$ cells were not largely sensitive to estragole albeit only by $\sim 20 \%$ ( $P=0.041$ and $P=0.038$, respectively) compared to untreated cells (Figure 4.9). 
These results suggest that FKS1 and more broadly cell wall synthesis are not the target of estragole.

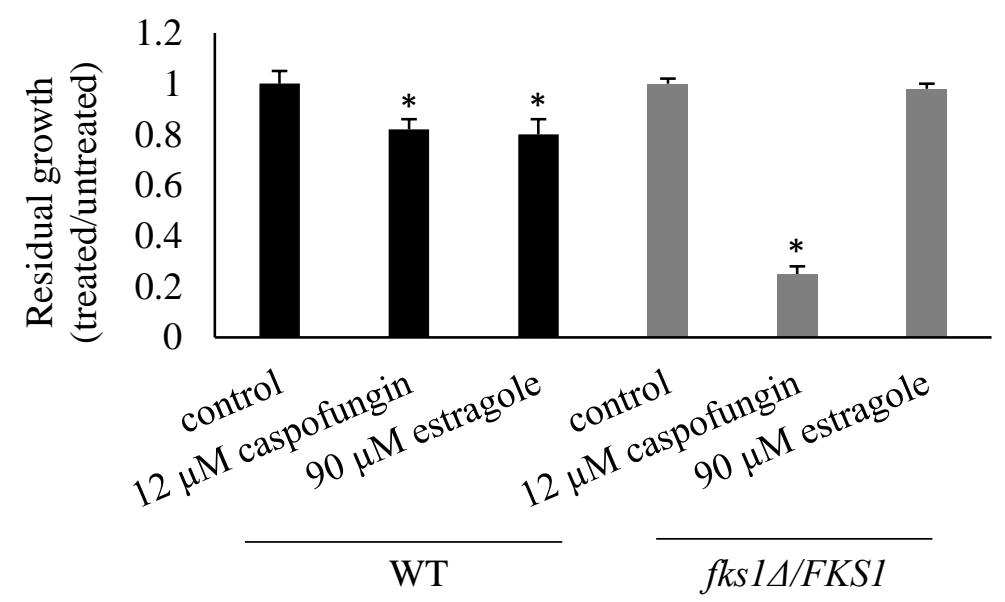

Figure 4-9. FKS1 is not the primary target of estragole. WT treatments compared to the relative WT control and $f k s 1 \triangle / F K S 1$ treatments compared to the relative control. Test was done in 96-well plate in triplicate and data are value mean \pm SD. Growth was measured by plate reader at 14 hours of growth at $\mathrm{OD}_{600}{ }^{*}, p<0.05$, Student's t-test comparison with relative control.

\subsubsection{The LCB1 target of myriocin is not the target of estragole}

Myriocin is an example of an antifungal drug targeting sphingolipids in the fungal plasma membrane (Chen, et al. 1999). Specifically, myriocin inhibits LCB1 and LCB2, two enzymes that catalyse the first and rate-limiting step in sphingolipid biosynthesis (Toume and Tani 2014). To determine if estragole has the same LCB1 target as myriocin, growth of WT and $l c b 1 \triangle / L C B 1$ in $125 \mu \mathrm{M}$ myriocin and $90 \mu \mathrm{M}$ estragole, concentrations that inhibit the growth of WT by $20 \%$, was compared to untreated cells. As previously observed (Toume and Tani 2014), $l c b 1 \Delta / L C B 1$ was significantly sensitive to myriocin with $70 \%$ inhibition $(P=$ 0.035) compared to untreated cells, and this sensitivity was not observed in WT cells (Figure 4.10). In contrast, both WT and $l c b 1 \triangle / L C B 1$ cells were not largely sensitive to estragole albeit only by $\sim 20 \%$ ( $P=0.04$ and $P=0.03$, respectively) compared to untreated cells (Figure 4.10). These results suggest that LCB1 and more broadly sphingolipids are not the target of estragole. 


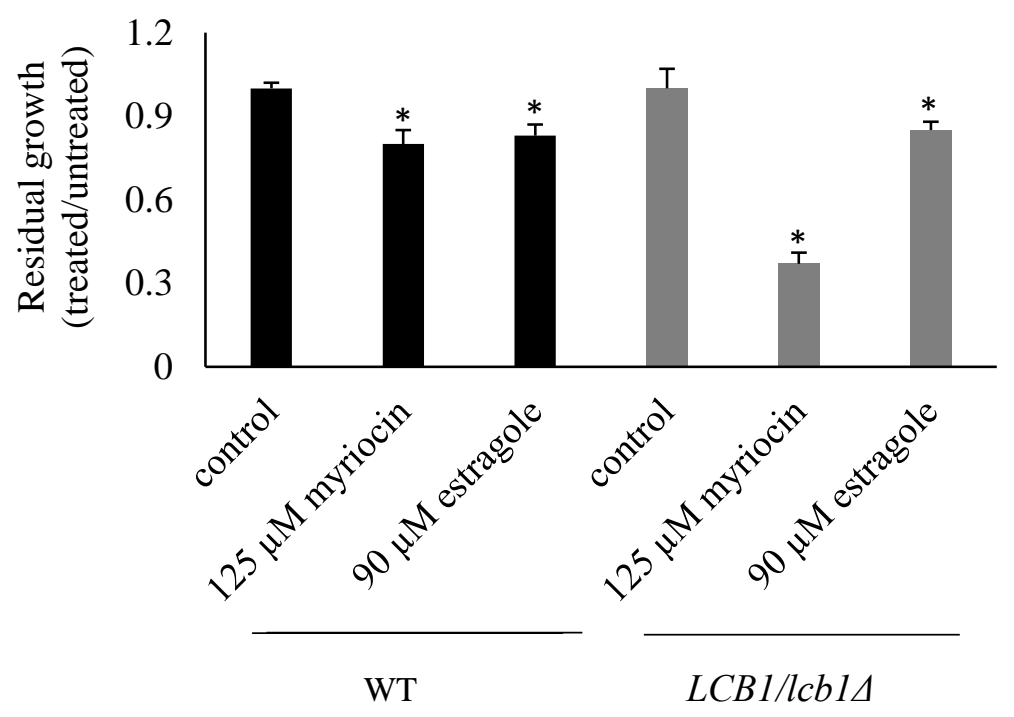

Figure 4-10. Estragole target is not LCB1. WT treatments compared to the relative WT control and $l c b 1 \triangle / L C B 1$ treatments compared to the relative control. Values are residual growth at 14 hours in triplicate in $\mathrm{SC}$ media measured at $\mathrm{OD}_{600}{ }^{*}, p<0.05$, Student's t-test comparison with relative control.

\subsubsection{The CHS1 target of polyoxin is not the target of estragole}

Polyoxin is an antifungal drug targeting chitin synthasis in the fungal cell wall (Endo, et al. 1970). Specifically, polyoxin inhibits the major chitin synthase gene CHS1 (Mehta, et al. 1984). To determine if estragole has the same CHS1 target as polyoxin, growth of WT and chs14/CHS1 in $0.9 \mu \mathrm{M}$ polyoxin and $90 \mu \mathrm{M}$ estragole, concentrations that inhibit the growth of WT by $20 \%$, was compared to untreated cells. As previously observed (Sburlati and Cabib 1986), chs $14 / C H S 1$ was significantly sensitive to polyoxin with $69 \%$ inhibition $(P=0.03)$ compared to untreated cells, and this sensitivity was not observed in WT cells (Figure 4.11). In contrast, both WT and chs $1 \triangle / C H S 1$ cells were not largely sensitive to estragole albeit only by $\sim 20 \%$ ( $P=0.03$ and $P=0.02$, respectively) compared to untreated cells (Figure 4.11). These results suggest that CHS1 and more broadly chitin are not the target of estragole. 


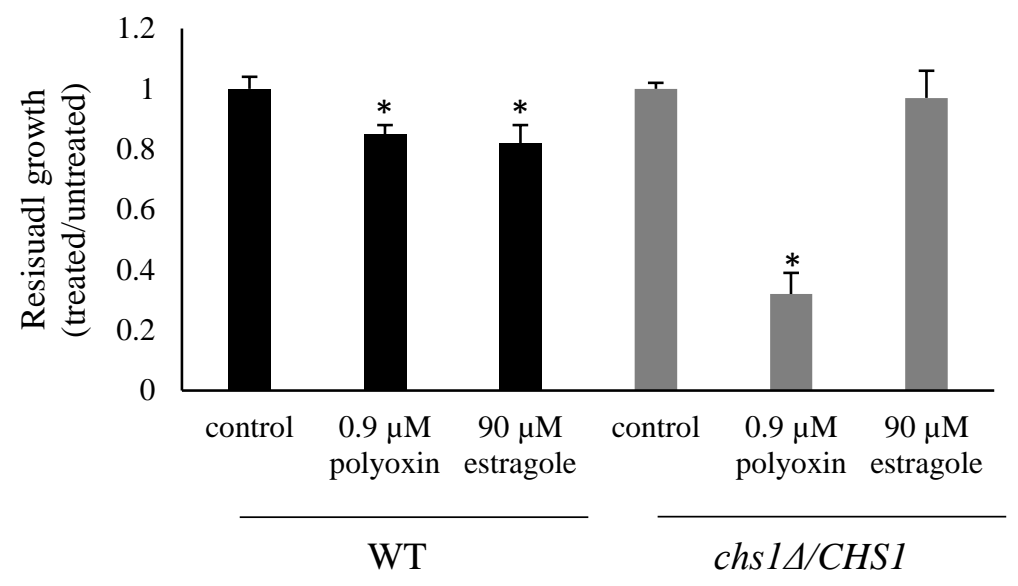

Figure 4-11. CHS1 is not the primary target of estragole. WT treatments compared to the relative WT control and chs $14 / C H S 1$ treatments compared to the relative control. Values are residual growth at 14 hours in triplicate in SC media measured at $\mathrm{OD}_{600}{ }^{*}, p<0.05$, Student's $t$-test comparison with relative control.

\subsubsection{Estragole mechanism is different from Flucytosine}

Flucytosine is an antifungal agent that inhibits biosynthesis of fungal DNA and RNA (Paluszynski, et al. 2008). The compound itself does not have antifungal activity but after entering to the cell, it is converted to the active form of drug that gets incorporated into RNA or inhibits the thymidylate synthesis during DNA biosynthesis. The deletion of FCY1 or FCY2 gene in S. cerevisiae cause flucytosine resistance (Paluszynski, et al. 2008; Vermes, et al. 2000). Thus, to further clarify if estragole mechanism is same as flucytosine, we have done the growth assay on $f c y l \Delta$ with flucytosine and estragole. While there is about 55\% inhibition in WT by both drugs, $f c y l \Delta$ was resistant to flucytosine up to residual growth of $96 \%$ but still sensitive to estragole. This result confirms target of estragole is not the flucytosine target. 


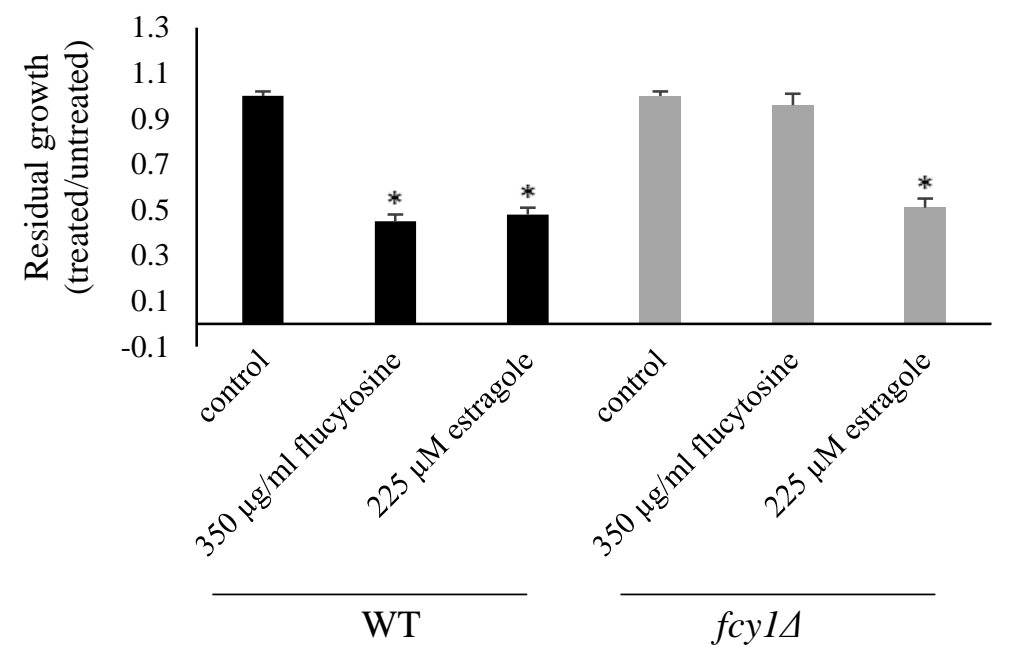

Figure 4-12. Estragole mechanism is distinct from flucytosine. WT treatments compared to the relative WT control and $f c y 1 \Delta$ treatments compared to the relative control. Values are residual growth at 14 hours in triplicate in $\mathrm{SC}$ media measured at $\mathrm{OD}_{600}$. ${ }^{*}, p<0.05$, Student's $t$-test comparison with relative control.

\subsubsection{Synergy of estragole and established antifungal drugs}

Drug combination therapy allows patients to take two drugs at once to get a better effect than taking either drug alone, which results in reduced doses of each drug and reduced rates in the evolution of drug resistance (Jia, et al. 2009). In addition, analyses of drug combinations provide insight into the pathways targeted by the drugs. For example, two drugs working in distinct pathways will exhibit synergy. In the case of antifungal drugs, two antifungal drugs targeting distinct pathways will confer greater inhibition than the sum of inhibition of each antifungal individually (Jia, et al. 2009; Slinker 1998). We thus exploited this concept of synergy to further discern the pathways targeted by estragole. Synergy of estragole with ketoconazole has previously suggested that the antifungal activity of estragole is through a mechanism distinct from ketoconazole (Shin and Kang 2003), thus it is warranted to look at additional antifungal pathways to further understand the antifungal mechanism of action of estragole. Here we evaluated synergy of estragole with antifungal drugs targeting various 
pathways. Growth of $S$. cerevisiae was measured in single and combinatorial treatments at concentrations where each drug inhibited the growth of WT by $15 \%$ compared to untreated cells (Figure 4.13 A). First, we examined synergy of estragole with drugs targeting ergosterol. Relative to the $15 \%$ inhibition conferred by the individual drugs, the combination treatments of estragole with ketoconazole, nystatin and amphotericin B conferred growth inhibition by 40, 45 and $50 \%$, respectively, compared to untreated cells. Second, we examined synergy of estragole with caspofungin and polyoxin that target $\beta$-(1,3)-glucan synthesis and chitin synthesis, respectively, in the fungal cell wall. Relative to the $15 \%$ inhibition conferred by the individual drugs, the combination treatment of estragole with caspofungin or estragole with polyoxin conferred growth inhibition by 33 and 30\%, respectively, compared to untreated cells. Third, we examined synergy of estragole with myriocin that targets sphingolipid homeostasis in the fungal plasma membrane. Relative to the $15 \%$ inhibition conferred by the individual drugs, the combination treatment of estragole with myriocin conferred growth inhibition by $25 \%$ compared to untreated cells. As there are different methods for synergy activity quantification, we applied two formulations to investigate synergy. In the first method, we calculated the combination index $(\mathrm{CI}=\mathrm{A}+\mathrm{B} / \mathrm{AB}$ when $\mathrm{A}$ is drug $\mathrm{A}$ effect, $\mathrm{B}$ is drug $\mathrm{B}$ effect and $\mathrm{AB}$ is mixture effect) as previously described (Foucquier and Guedj 2015). Synergy is detected when CI is less than 1. Ketoconazole, nystatin, amphotericin B and caspofungin exhibited synergy with estragole with CI values of $0.6,0.68,0.72$ and 0.82 , respectively. In the other formulation that incorporates the MIC, we calculated the fractional inhibitory concentration (FIC) index where as described before (Meletiadis, et al. 2010), FIC= (AMIC in mix/ AMIC alone) + (BMIC in mix/ BMIC alone). Synergy is detected when FIC is less than 0.5. Only ketoconazole and amphotericin B exhibited synergy with estragole with FIC values of 0.46 and 0.5 , respectively. Among the 3 different methods, we prefer to agree with the FIC calculation that is most cautious among the three calculations. These results indicate that synergy was only achieved with the ketoconazole and amphotericin B antifungal drugs targeting 
ergosterol, which would be consistent with our results above showing that estragole is not targeting ergosterol.

A

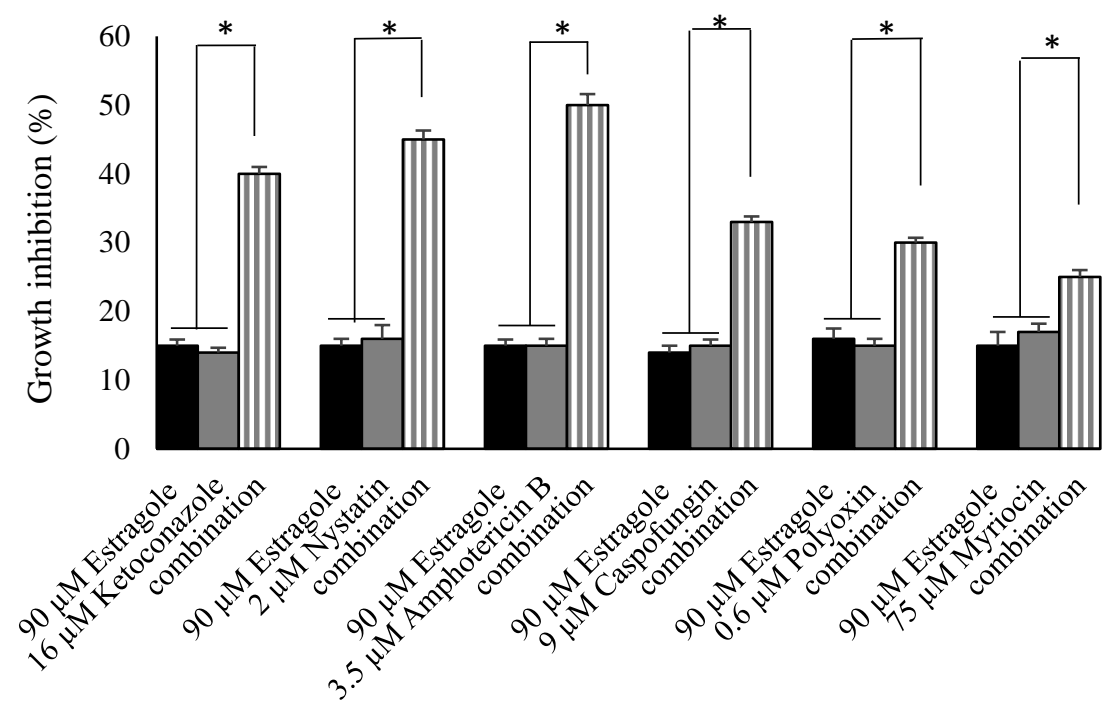

B

combinatory with $90 \mu \mathrm{M} \quad$ CI $\quad$ effect by CI FIC $\quad$ effect by FIC estragole

\begin{tabular}{lllll}
\hline $16 \mu \mathrm{M}$ ketoconazole & 0.61 & synergy & 0.46 & synergy \\
\hline $2 \mu \mathrm{M}$ nystatin & 0.68 & synergy & 0.72 & no interaction \\
\hline $3.5 \mu \mathrm{M}$ AmN & 0.72 & synergy & 0.5 & synergy \\
\hline $9 \mu \mathrm{M}$ caspofungin & 0.87 & synergy & 1.11 & no interaction \\
\hline $0.6 \mu \mathrm{M}$ polyoxin & 1.03 & additive & 1.75 & no interaction \\
\hline $75 \mu \mathrm{M}$ myriocin & 1.28 & antagonistic & 1.92 & no interaction \\
\hline
\end{tabular}

Cl<1 synergy $\mathrm{Cl}>1$ antagonist $\mathrm{Cl}=1$ no interaction

$\mathrm{FIC}<0.5$ synergy $0.5<\mathrm{FIC}<4$ no interaction FIC $>4$ antagonist

Figure 4-13. Combinatorial effect of estragole with known antifungals. Cells were grown to mid-log phase in presence of estragole and each of known antifungals. *, $p<0.05$, Student's t-test comparison with relative control. A) classic combination result without calculation. B) synergy effect quantification using two different formulations. 


\subsubsection{HIP-HOP analysis to determine the mechanism of action of estragole}

Our results thus far suggest that the antifungal mechanism of action of estragole is different from other known antifungal agents. To elucidate a mechanism of action in an unbiased manner, we conducted a HIP-HOP analysis to identify heterozygote mutant growth defects and homozygote mutant growth defects in the presence and absence of estragole (Robinson, et al. 2014). The objective of the HIP analysis is to identify the main target of estragole, while the objective of the HOP analysis is to identify the genes buffering the effect of estragole.

\subsubsection{HIP analysis of estragole}

To identify the direct target of estragole, the heterozygous deletion library pool was grown in the presence and absence of $90 \mu \mathrm{M}$ estragole, a concentration that reduced the growth of the pooled library by $20 \%$ compared to the untreated pool . Pools were grown until mid-log, at which time genomic DNA was extracted and barcodes flanking deletions (UPTAG and DNTAG) were quantified using next-generation sequencing. The sequencing quality (logCPM) was compared with fold change in barcode abundance in treated compared to untreated conditions $(\log \mathrm{FC}$ ) for 5,928 strains (Figure 4.14), which indicated $\log \mathrm{FC}$ values between -4.5 and 3.9 and $\log$ CPM values primarily between 2.6 and 9.7. These distributions are representative of reasonable growth variation in response to estragole; however, not one statistically significant change comparing barcode abundance in treated and untreated conditions was detected based on a standard cutoff (FDR $<0.05)$. 


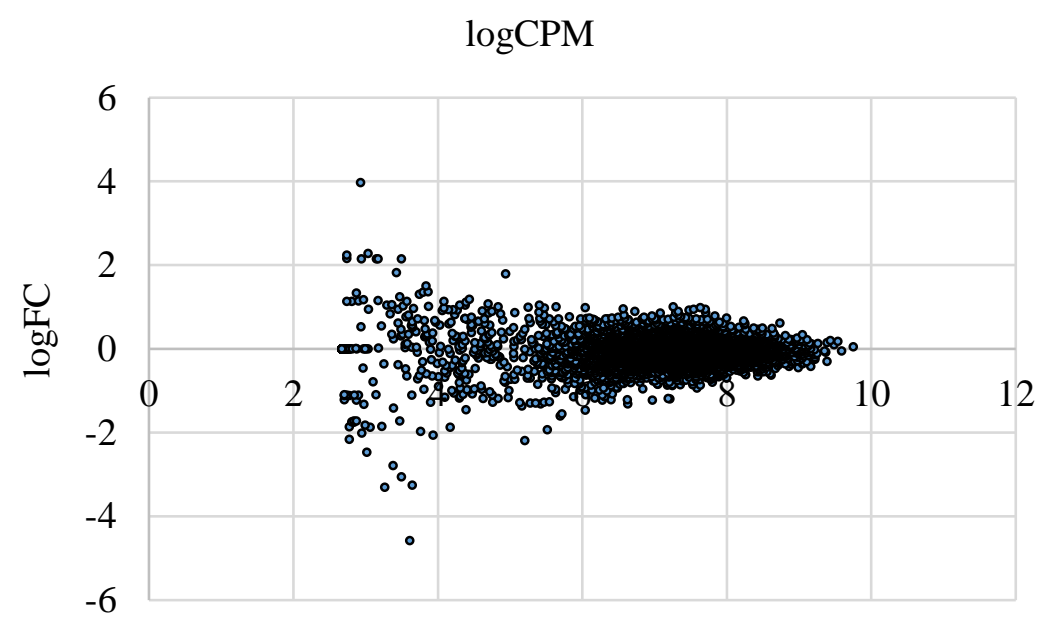

Figure 4-14. Fitness plot of HET library in presence of estragole. Read counts were compared in treatment with control. Higher $\operatorname{logFC}$ indicates more changes in read counts while negative values mean mutants grew less and positive values show mutants grew better. $\log \mathrm{CPM}>3$ indicates high quality of sequencing.

As statistical significance did not identify any strains to validate, we alternatively selected to validate 80 strains based on growth inhibition $>35 \%$ in the average number of barcode reads for each strain in control and treated conditions. Growth was measured in an independent assay that was not confounded by the competitive aspect of the pooled strains. Growth of specific strains was compared in treated and untreated media, and residual growth was calculated by the ratio of growth in treated to untreated media. Six of the 80 strains exhibited significant growth defects compared to the WT strain (Figure 4.15); all strains exhibited $30-45 \%$ growth defects with the estragole treatment compared to the $20 \%$ growth defect in WT. These results indicate that the processes of protein kinase (KIN3), transmembrane sorting receptor and cycle between golgi and endosome (PEP1), high affinity uridine permease (FUI1), Component of the DNA ligase IV complex (LIF1) and transcriptional regulator (SUB1), likely buffer the mechanism of estragole, as the sensitivity detected here was not indicative of of hypersensitivity (>60\% inhibition) expected for drug targets. 


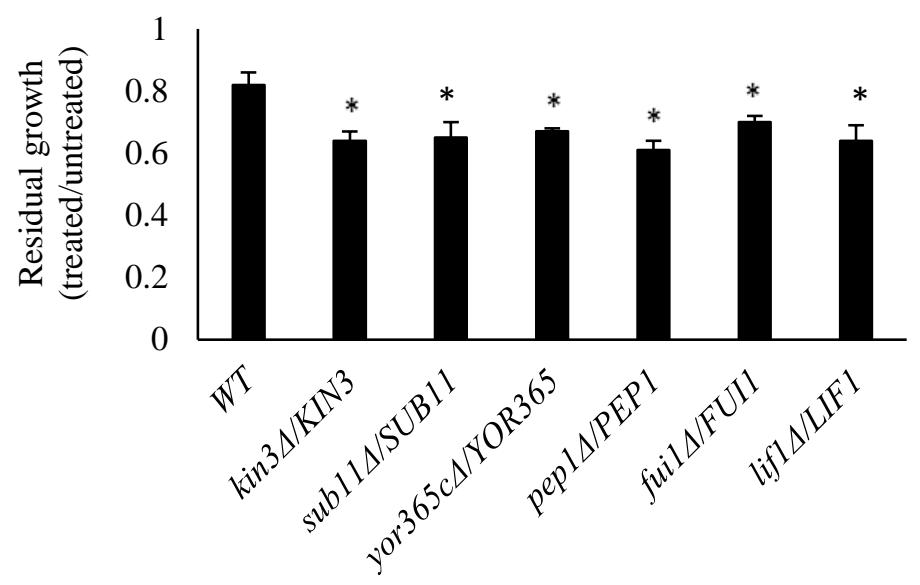

Figure 4-15. Validation of residual growth of HET sensitive deletion strains in presence of estragole compared to WT strain. The growth was measured by $\mathrm{OD}_{600}$ absorbance reading at 14 hours and compared to WT growth at the same time. ${ }^{*}, p<0.05$ in comparison to WT.

Table 4-2. Annotation of sensitive HET deletion strains in presence of estragole.

\begin{tabular}{lll}
\hline $\begin{array}{l}\text { Systematic } \\
\text { name }\end{array}$ & Gene & Biological function \\
\hline YAR018C & KIN3 & $\begin{array}{l}\text { Possible role in DNA damage response; influences tolerance to } \\
\text { high levels of ethanol }\end{array}$ \\
\hline YMR039C & SUB1 & $\begin{array}{l}\text { Role in nonhomologous end-joining (NHEJ) of ds breaks in } \\
\text { plasmid DNA, but not chromosomal DNA }\end{array}$ \\
\hline YOR365C & YOR365C & Putative protein of unknown function \\
\hline YBL017C & PEP1 & $\begin{array}{l}\text { Cycles between the late-Golgi and prevacuolar endosome-like } \\
\text { compartments }\end{array}$ \\
\hline YBL042C & FUI1 & $\begin{array}{l}\text { High affinity uridine permease, localizes to the plasma } \\
\text { membrane }\end{array}$ \\
\hline YGL090W & LIF1 & $\begin{array}{l}\text { Mediates nonhomologous end joining in DNA double-strand } \\
\text { break repair }\end{array}$ \\
\hline
\end{tabular}




\subsubsection{HOP analysis of estragole}

To complement the HIP analysis, we used the same method to conduct a HOP analysis to identify genes buffering the mechanism of estragole. Pools of the homozygous deletion library pool was grown in the presence and absence of $90 \mu \mathrm{M}$ estragole, a concentration that reduced the growth of the pooled homozygous mutant library by $20 \%$ compared to the untreated pool . Pools were grown until mid-log, at which time genomic DNA was extracted and barcodes flanking deletions (UPTAG and DNTAG) were quantified using next-generation sequencing. The sequencing quality $(\log \mathrm{CPM})$ was compared with fold change in barcode abundance in treated compared to untreated conditions ( $\operatorname{logFC}$ ) for 5,928 strains (Figure 4.16), which indicated $\log \mathrm{FC}$ values between -4.4 and 3.7 and $\log \mathrm{CPM}$ values primarily between 1.4 and 11.3. These distributions are representative of reasonable growth variation in response to estragole. Unlike the HIP analysis, statistically significant changes in barcode abundance in treated and untreated conditions were detected in 42 strains (FDR $<0.05)$. In addition to the 42 significant differences, we identified 97 strains with $>65 \%$ differences in barcode abundance in treated and untreated media. 


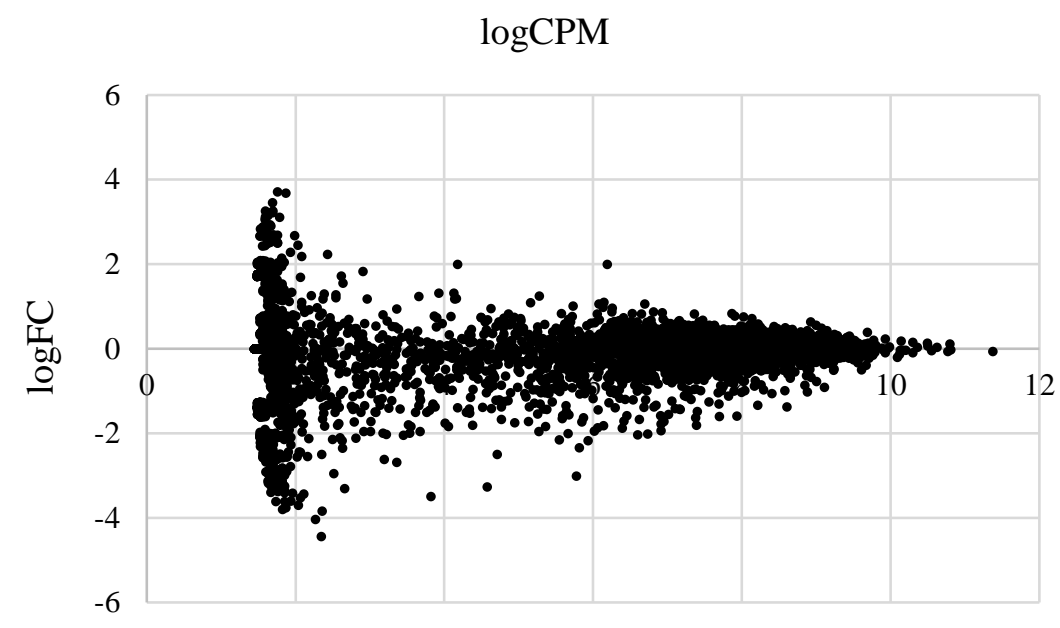

Figure 4-16. Fitness plot of HOM library in presence of estragole. Read counts were compared in treatment with control. Higher $\operatorname{logFC}$ indicates more changes in read counts while negative values mean mutants grew less and positive values show mutants grew better. LogCPM > 3 shows high quality of sequencing.

To validate these putatively sensitive strains, growth was measured in an independent assay that was not confounded by the competitive aspect of the pooled strains. Growth of 139 specific strains was compared in treated and untreated media, and residual growth was calculated by the ratio of growth in treated to untreated media. These results demonstrated that 21 of the 139 strains exhibited significant growth defects compared to the WT strain (Figure 4.17); these 21 strains exhibited 30-48\% growth defects with the estragole treatment compared to the $15-20 \%$ growth defect in WT. These results indicate that the processes of mitochondrial ribosomal proteins (RPS16A, MRPL20, MRP51, MRPL51, SWS2, RPL7A, RPS16A, RPL13B), mitochondrial function and energy metabolism disruption (MRP10, GGC1, RRG1, CEM1, FMP33, ATP12, MRPL20, PPA2, SWS2, ATP11, MDM12, MRP51, MRPL51), nonDNA binding transcription (RTF1), polyamine biosynthesis (SPE1) and an unknown gene YMR135W buffer the mechanism of estragole (Table 4.3).

To gain more insight into these 21 genes, we tested for enrichment in three Gene Ontology categories. There was not significant enrichment for biological processes and 
molecular functions, while there was enrichment for the cellular component category. Eight genes were components of ribosomal subunits (RPS16A, MRPL20, MRP51, MRPL51, SWS2, RPL7A, RPS16A, RPL13B) and 13 genes were components of mitochondrial subunits (MRP10, GGC1, RRG1, CEM1, FMP33, ATP12, MRPL20, PPA2, SWS2, ATP11, MDM12, MRP51, MRPL51), of which eight genes are involved in mitochondrial function. One of these genes, SWS2, was identified as the most hypersensitive gene to mitochondrial stress (Lee, et al. 2014). These results suggest that mitochondrial activity may buffer the primary mechanism of estragole.

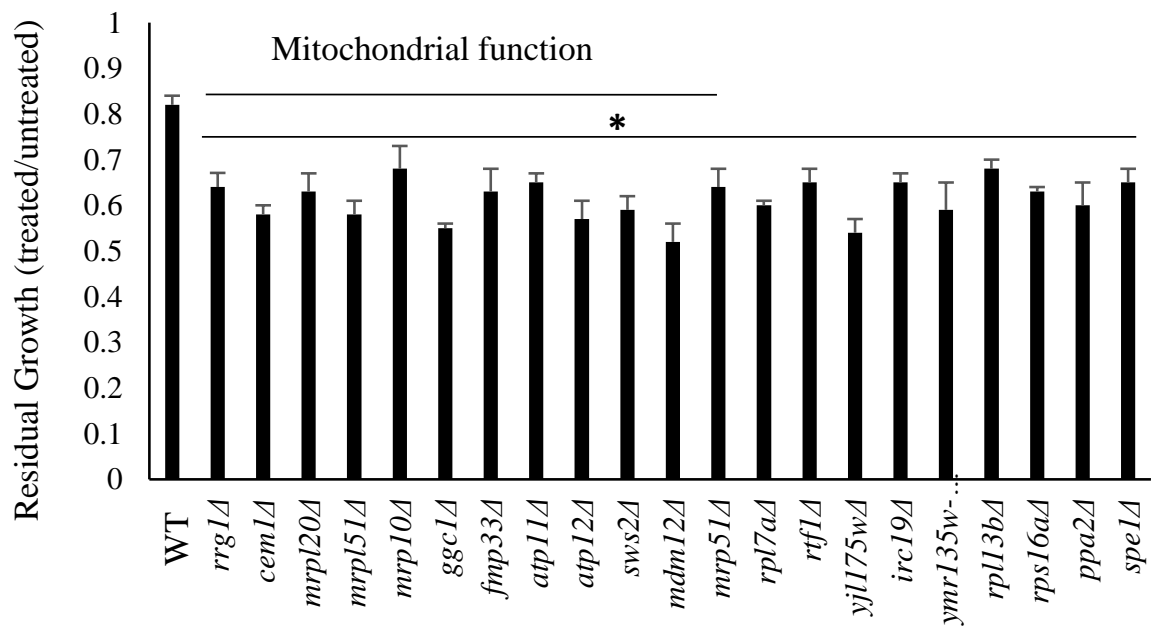

Figure 4-17. Validation of residual growth of sensitive mutants in presence of estragole compared to WT strain. The growth was measured by $\mathrm{OD}_{600}$ absorbance reading at 14 hours and compared to WT growth at the same time. Values are triplicate average when ${ }^{*}, p<0.05$, Student's $t$-test comparison with WT. 
Table 4-3. Annotation of sensitive deletion strains in presence of estragole in HOM library.

\begin{tabular}{|c|c|c|}
\hline $\begin{array}{l}\text { Systematic } \\
\text { name }\end{array}$ & Gene & Biological function \\
\hline YNL315C & ATP11 & ATP synthase \\
\hline YJL180C & ATP12 & ATP synthase \\
\hline YER061C & CEM1 & $\begin{array}{l}\text { Condensing Enzyme with Mitochondrial } \\
\text { function }\end{array}$ \\
\hline YJL161W & FMP33 & Unknown (Mitochondrial Proteome) \\
\hline YDL198C & GGC1 & GDP/GTP Carrier \\
\hline YLL033W & IRC19 & Unknown (increased Recombination Centers) \\
\hline YDR017C & KCS1 & pKC1 Suppressor \\
\hline YOL009C & MDM12 & Mitochondrial Distribution and Morphology \\
\hline YDL045W-A & MRP10 & Mitochondrial Ribosomal Protein \\
\hline YPL118W & MRP51 & Mitochondrial Ribosomal Protein \\
\hline YKR085C & MRPL20 & Mitochondrial Ribosomal Protein, Large subunit \\
\hline YPR100W & MRPL51 & Mitochondrial Ribosomal Protein, Large subunit \\
\hline YDR140W & MTQ2 & Methyltransferase \\
\hline YMR267W & PPA2 & PyroPhosphatAse \\
\hline YMR142C & RPL13B & Ribosomal Protein of the Large subunit \\
\hline YGL076C & RPL7A & Ribosomal Protein of the Large subunit \\
\hline YMR143W & RPS16A & Ribosomal Protein of the Small subunit \\
\hline YDR065W & RRG1 & Required for Respiratory Growth \\
\hline YGL244W & RTF1 & chromatin remodeling Paf1 complex \\
\hline YBL007C & SLA1 & Cytoskeletal protein \\
\hline YKL184W & SPE1 & Spermidine auxotroph \\
\hline YNL081C & SWS2 & mitochondrial ribosomal protein \\
\hline YJL175W & YJL175W & Unknown \\
\hline YMR135W-A & YMR135W-A & Unknown \\
\hline
\end{tabular}

In addition to 21 homozygous mutations that increased sensitivity to estragole, there were 19 homozygous mutations that reduced sensitivity to estragole. The residual growth of these resistant strains were $1.1-1.8$, values that were significantly greater than the $0.8-0.85$ values in WT (Figure 4.18). Functional analyses of these 22 genes revealed only one significantly over-represented process, iron transport, suggesting that estragole results in an iron overload in the cell, which could explain increased growth in strains deficient in iron transporters. The 19 resistant strains are shown in Table 4.4 with their biological functions. 


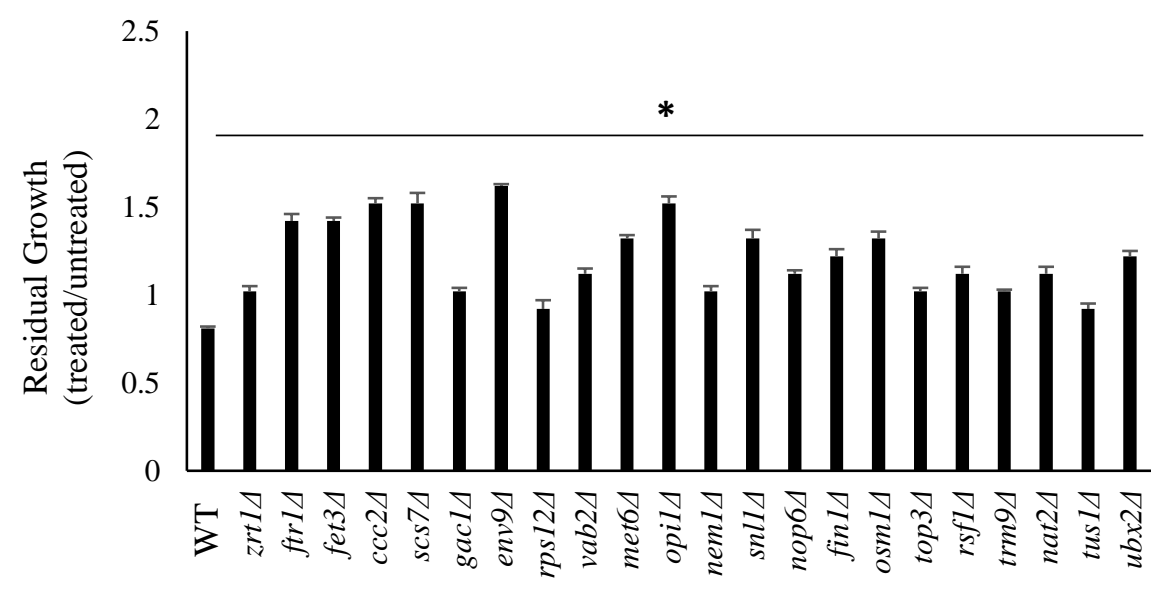

Figure 4-18. Validation of residual growth of 19 hits grew better in presence of estragole compared to WT strain. The growth was measured by $\mathrm{OD}_{600}$ absorbance reading at 14 hours and compared to WT growth at the same time. Values are triplicate average. ${ }^{*}, p<0.05$, Student's $t$-test comparison with WT.

Table 4-4. Annotation of deletion strains grew better in presence of estragole in HOM library.

\begin{tabular}{lll}
\hline Systematic name & Gene & Biological function \\
\hline YDR270W & CCC2 & Cross-Complements Ca(2+) phenotype of csg1 \\
YOR246C & ENV9 & Late endosome and vacuole interface function \\
YMR058W & FET3 & Fe Transporter \\
YDR130C & FIN1 & Filaments In between Nuclei \\
YER145C & FTR1 & Fe TRansporter \\
YOR178C & GAC1 & Glycogen ACcumulation \\
YER091C & MET6 & Methionine requiring \\
YHR004C & NEM1 & Nuclear Envelope Morphology \\
YDL213C & NOP6 & Nucleolar Protein \\
YHL020C & OPI1 & OverProducer of Inositol \\
YJR051W & OSM1 & Osmotic sensitivity \\
YOR369C & RPS12 & Ribosomal Protein of the Small subunit \\
YMR272C & SCS7 & Suppressor of Ca2+ Sensitivity \\
YIL016W & SNL1 & Suppressor of Nup116-C Lethal \\
YLR234W & TOP3 & Topoisomerase \\
YML014W & TRM9 & T-RNA Methyltransferase \\
YML013W & UBX2 & Ubiquitin regulatory X \\
YEL005C & VAB2 & Endosomal maturation \\
YGL255W & ZRT1 & Zinc-Regulated Transporter \\
\hline
\end{tabular}




\subsubsection{Iron and zinc supplementation does not rescue estragole-induced growth reduction}

As iron transporter deletions $(f e t 3 \Delta, f t r 1 \Delta, \operatorname{ccc} 2 \Delta)$ and zinc transporter deletion (zrt1A) showed better growth with estragole treatment, we set up an experiment to investigate how the estragole mechanism involves metal homeostasis. WT (BY4741) cells were treated in the presence of different estragole concentrations with the addition of various levels of iron $\left(\mathrm{FeCl}_{3}\right)$ and zinc $\left(\mathrm{ZnCl}_{2}\right)$. Increasing concentrations of iron or zinc generally resulted in increased toxicity of estragole (Figure 4.19). Significantly increased toxicity of $90 \mu \mathrm{M}$ estragole was observed with $100 \mu \mathrm{M} \mathrm{FeCl}_{3}$, while significantly increased toxicity of 250 or $400 \mu \mathrm{M}$ estragole was observed with $50 \mu \mathrm{M} \mathrm{FeCl}_{3}$. Likewise, significantly increased toxicity of 90 or $250 \mu \mathrm{M}$

estragole was observed with $25 \mu \mathrm{M} \mathrm{ZnCl}$, while the toxicity of $400 \mu \mathrm{M}$ was not impacted with zinc supplementation. These results indicate that not only metal supplementation did not rescue the estragole-mediated growth defect, but also exacerbated the growth defect by estragole, thus estragole is not likely a metal chelator but likely causing metal toxicity.

A

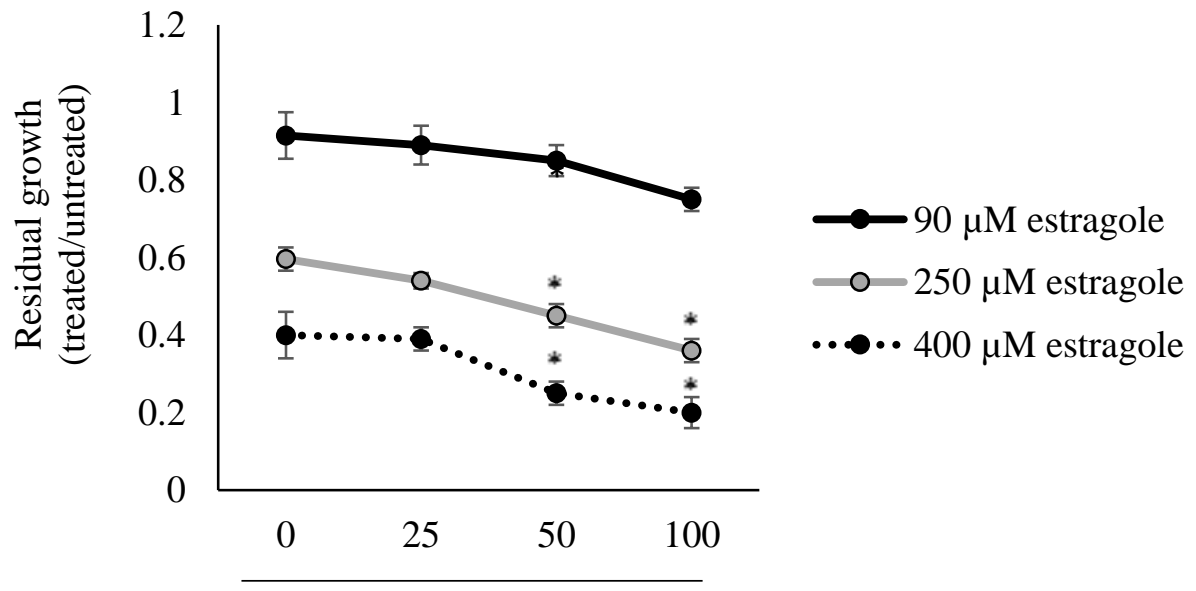

$\mathrm{FeCl}_{3}(\mu \mathrm{M})$ 


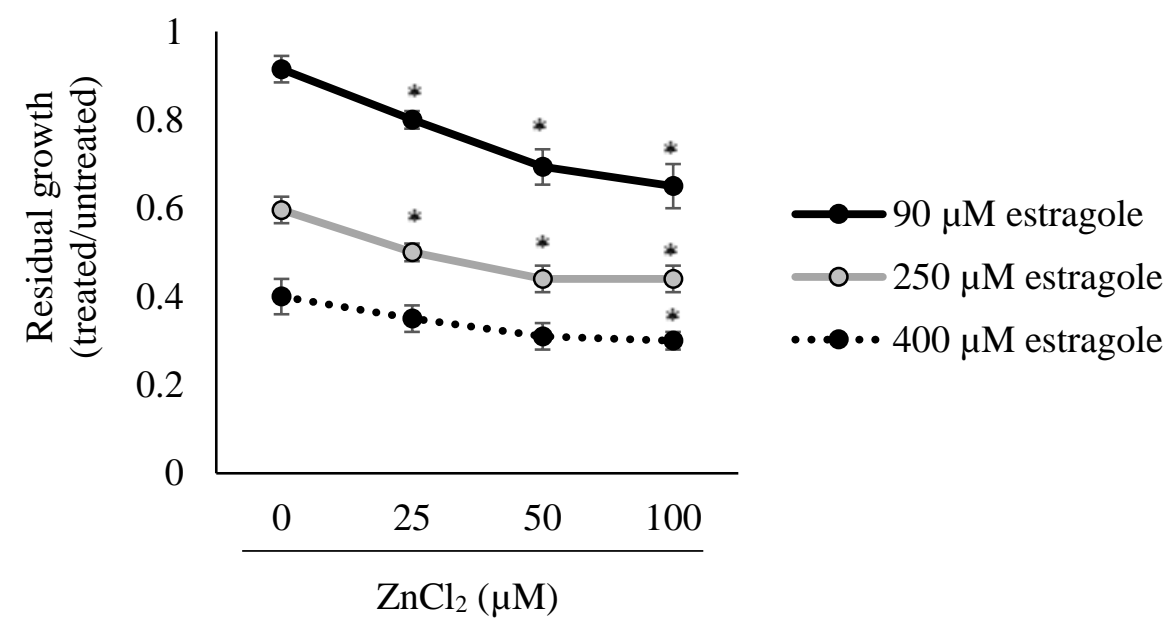

Figure 4-19. Iron and Zinc exacerbate the inhibition by estrogole. A) Iron supplementation with 3 different concentrations reduces the residual growth by estragole. ${ }^{*}, p<0.05$, Student's t-test comparison with no iron condition. B) Zinc supplementation with 3 different concentrations exacerbates inhibition by estragole. Growth was measured after 14 hours by plate reader at $\mathrm{OD}_{600}$ * , $p<0.05$, Student's $t$-test comparison with no zinc condition.

\subsubsection{Estragole treatment induces oxidative stress}

To further investigate the sensitivity of mitochondrial deletion strains, oxidative stress was investigated in response to estragole treatment. Glutathione is an established metabolite that ameliorates oxidative stress (Birben, et al. 2012). We treated WT cells with estragole and glutathione separately as well as together to determine if glutathione can rescue the estragoleinduced growth defect (Figure 4.20). Estragole (120 uM) induced a 66\% growth defect, while glutathione $(1 \mathrm{mM})$ did not significantly impact growth. Interestingly, the glutathione treatment ameliorated the estragole growth defect from 66\% (estragole alone) to $19 \%$ (co-treatement of estragole and glutathione). These results suggest that estragole induces oxidative stress, perhaps via reactive oxygen species (ROS). 


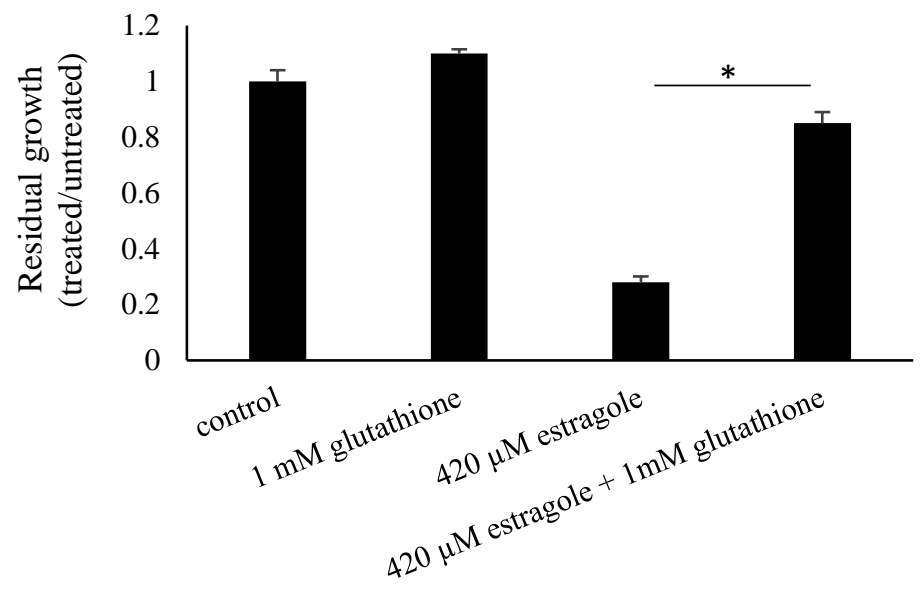

Figure 4-20. Glutathione rescues the estragole ROS level increase. Cells were treated for 14 hours and growth was measure by plate reader at $\mathrm{OD}_{600}$. Data are mean $\pm \mathrm{SD} .{ }^{*}, p<0.05$, Student's $t$-test.

\subsubsection{Estragole disrupts mitochondrial morphology}

To further investigate the impact of estragole on mitochondrial function, Mitotracker Red was used to assess mitochondrial morphology (Figure 4.21). In the control, Mitotracker Red was localized to elongated tubular network normally (Okamoto and Shaw 2005). In contrast, the estragole treatment resulted in localization to fragmented/large round mitochondria. These results are consistent with FCCP (proton ionophore carbonyl cyanide-4(trifluoromethoxy)phenylhydrazone) treatment that is known to induce oxidative stress (Hughes, et al. 2016). These results suggest estragole induces oxidative stress, perhaps via increased ROS. 


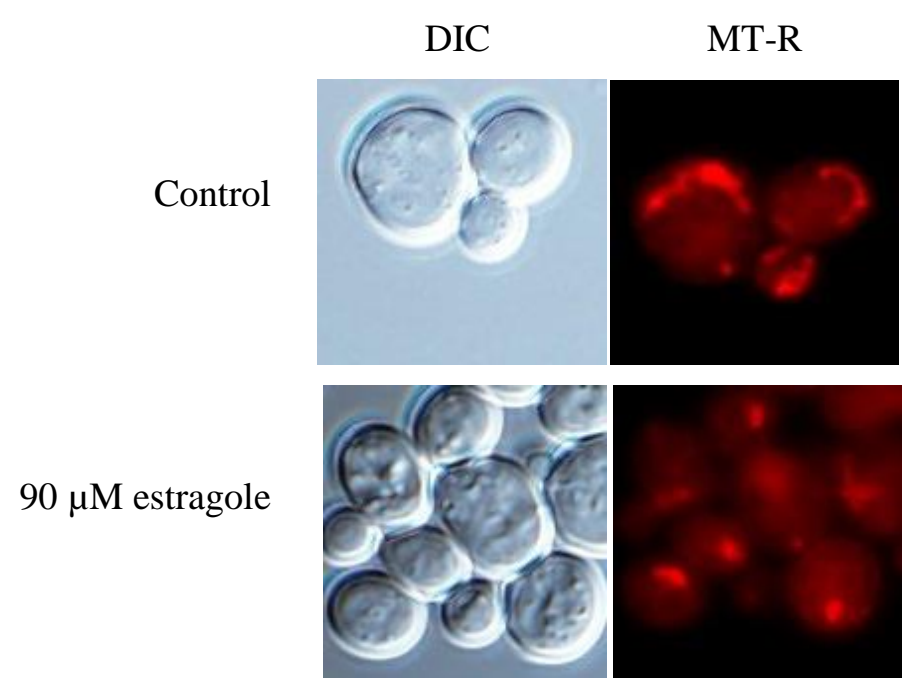

Figure 4-21. Estragole modifies the mitochondrial morphology. Cells were grown and treated to mid-log and stained with MitoTracker-Red (MT-R) for $30 \mathrm{~min}$. Images were taken by the Olympus fluorescent microscope at 100X using the Texas Red filter.

\subsection{DISCUSSION}

In this chapter, we aimed to identify the antifungal mechanism of action of estragole. Antifungal activity was confirmed with growth inhibition of pathogenic Candida species. Via comparisons with targets of existing antifungal agents, we determined the target of estragole is not a target of existing antifungal agents. These results suggest the mechanism might be distinct from known antifungal drugs. To elucidate this unsuspected mechanism, we conducted genome-wide analyses that quantified the abundance of homozygous and heterozygous gene deletions in the presence and absence of estragole.

Our demonstration that estragole was antifungal against pathogenic Candida species was largely consistent with previous studies of estragole (Andrade, et al. 2015). Antifungal activity of estragole was also shown in candida species and also illustrated the synergy effect with ketoconazole (Shin and Pyun 2004; Waller, et al. 2017) It was also shown that estragole inhibits other species such as Cryptococcus spp, Microsporum spp and also Trichophyton (Waller, et al. 2017). Despite all these studies, the antifungal mechanism is not known. Our 
report that heterozygous gene deletions known to be sensitive to other antifungal drugs are not hypersensitive to estragole indicates these genes are not the targets of estragole. However, there were four heterozygous gene deletions ( $h m g 1 \Delta / H M G 1$, erg1 $1 / E R G 1$, erg2 $/ E R G 2$, erg9 9 /ERG9) that were slightly sensitive to estragole, suggesting the mechanism of action of estragole includes an aspect of disrupted ergosterol metabolism. Some aspect of disrupted ergosterol metabolism may underlie the previous finding of synergy between estragole and ketoconazole (Shin and Kang 2003).

To elucidate the physical target of estragole, we performed a HIP analysis that identified six heterozygous deletion strains sensitive to estragole. As these strains were not haploinsufficient (hypersensitive), it is likely that the proteins encoded by these six genes are not the physical targets of estragole. However, the HIP assay is highly sensitive to the drug concentration based on previous reports that the ERG11 target of fluconazole was only detected with only $3 \%$ background inhibition of the HET library, while ERG11 was not identified as the target with 12 and 58\% background inhibition (Lee, et al. 2014). Since ERG11 was among the sensitive but not hypersensitive deletion strains with $20 \%$ background inhibition, it is plausible that one of the six sensitive strains are the estragole target, but this can only be deciphered by conducting the HIP assay at various estragole concentrations. Among the six candidate targets (KIN3, SUB1, YOR365C, PEP1, FUI1, LIF1), three (SUB1, LIF1, KIN3) are all involved in DNA damage repair, thus the estragole target might be one of these proteins or alternatively a protein upstream of these DNA damage repair proteins.

In the HOP analysis, homozygous deletions of ribosomal subunits and genes involved in mitochondrial function were sensitive. These results are related as mitochondrial energy metabolism, protein metabolism and respiration require 50 ribosomal subunits (Graack and Wittmann-Liebold 1998). Mitochondrial function is known to be the antifungal target of experimental compounds such as T-2307 (Shibata, et al. 2012). As these were results in our HOM analysis, we assume mitochondrial function is a secondary target of estragole rather than 
the main target. As mitochondria produce ROS during ATP metabolism, any defect in this organelle will result in increased ROS levels (Andreyev, et al. 2005; Perrone, et al. 2008). Our demonstration that glutathione rescued estragole antifungal activity as well as altered mitochondrial morphology with estragole treatment would be consistent with bioactivity through mitochondria and ROS. Increased ROS and mitochondrial dysfunction have previously been reported as antifungal mechanisms of many antifungal compounds (Delattin, et al. 2014; Hwang, et al. 2012), which would be an additional reason this mechanism is unlikely to be the primary and specific antifungal mechansim of estragole.

There were some extremely interesting results given the result in Chapter 3 that identified iron chelation as an antifungal mechanism of EtOH-vescalagin. Whereas growth of iron metabolism gene deletions was reduced with treatment of the iron chelator EtOHvescalagin, growth of three iron metabolism gene deletions (ftr $1 \Delta, f e t 3 \Delta, \operatorname{ccc} 2 \Delta)$ was increased with estragole treatment. Based on our results that iron supplementation exacerbated estragole bioactivity and deletion strains of iron transporters reduced estragole bioactivity, it is possible that estragole induces iron toxicity in the cell. Iron toxicity is an established toxic mechanism in humans which affects heart and liver by blood cells disorders as well as yeast model organism but has not been studied well as an antifungal mechanism (Shander, et al. 2009; Wiśnicka, et al. 1996). The iron toxicity mechanism would be consistent with mitochondrial DNA damage (Gao, et al. 2009). This thesis would be the first report of iron toxicity mediating the bioactivity of estragole.

The iron toxicity may likely be upstream of the mitochondrial damage based on our observations of two mitochondrial gene deletions. The MDM12 gene is required for normal mitochondrial inheritance and morphology, and with estragole treatment, the MDM12 deletion was significantly sensitive to estragole. Interestingly, the MDM12 morphology is altered from tubular mitochondria to large spherical mitochondria that causes interruption of transmission of mitochondria to daughter cells (Berger, et al. 1997). The SWS2 gene encodes a 
mitochondrial ribosomal subunit, and with estragole treatment, the SWS2 deletion was significantly sensitive. This result is consistent with growth defects of $s w s 2 \Delta$ in response to mitochondrial stress (Breker, et al. 2013; Lee, et al. 2014). Alternatively, it is plausible that mitochondrial stress precedes the iron toxicity.

Although it is still not clear if increased ROS is one of the first consequences of estragole treatment, ROS accumulation causes several damages to organelles, lipids and DNA. It is thus possible that high ROS levels lead to mitochondria DNA damage (Blasiak, et al. 2013), ultimately resulting in mitochondrial dysfunction by estragole. The other possibility is that the DNA damage repair system can be the downstream target of estragole which might cause high DNA damage and mitochondrial dysfunction that results in high level of ROS. Further work that considers the mechanisms of iron toxicity, ROS, and DNA damage will be critical to further understand the mechanism of action of estragole. For example, iron and ROS are both significant mediators of cell death (Dixon and Stockwell 2014).

In summary, we further investigated the antifungal activity of estragole. Consistent with previous reports (Khan, et al. 2011), we demonstrated a role for oxidative stress and mitochondrial dysfunction in estragole bioactivity. In addition, the chemical genetic analyses implicated specific genes in these processes. Notably, the unbiased approach of the HIP-HOP analysis identified that estragole bioactivity is accomplished via iron toxicity. Although the physical target of estragole is distinct from known antifungal agents, the mechanism may include disrupted ergosterol metabolism in common with known antifungals. As the potency of estragole $(>90 \mu \mathrm{M})$ is shy of the potency expected for a human therapeutic, there is still potential for estragole as an antifungal agent in the agricultural sector, particularly since estragole has been conserved as an active metabolite in a plant for millions of years. 



\section{Chapter 5 Implications and future directions}

\subsection{SUMMARY}

In this thesis, we assessed the chemical diversity of potential antifungal compounds derived from feijoa fruit. In Chapter 2, we investigated 16 cultivars of feijoa fruit using bioactivity-guided metabolite profiling of cultivars. Using GC-MS and multivariate analysis, we correlated compound profiles to bioactivity and detected the most potent cultivars and compounds, of which 4-cyclopentene-1,3-dione was the most potent antifungal compound with an $\mathrm{IC}_{50}$ of $\sim 1 \mu \mathrm{M}$ against human pathogenic fungi. In chapter 3 , we assessed the antifungal activity of EtOH-vescalagin from feijoa fruit, which showed antifungal activity with nanomolar potency against human pathogenic Candida species. Utilizing chemical genetics, we identified extracellular iron as a target of EtOH-vescalagin causing a decrease in intracellular iron levels, resulting in defects in zinc ion homeostasis and retromer function. In chapter 4, we focused on estragole, a lesser studied compound from feijoa fruit with reduced antifungal activity $\left(\mathrm{IC}_{50}\right.$ $\sim 100 \mu \mathrm{M}$ ) albeit interacting with a distinct target from established antifungal drugs, suggesting estragole belongs to a new class of antifungal compounds.

Feijoa bioactivity has been studied previously to some extent, although most studies have been done on only one feijoa cultivar, and reported some effect of crude methanolic, acetonic, or ethanolic extracts (Basile, et al. 2010; Bontempo, et al. 2007; Elmastaş and Gedikli 2010; Karami, et al. 2013; Lapčík, et al. 2005; Nasef, et al. 2015; Tuncel, et al. ; Turco, et al. 2016; Varga and Molnar 2000). In contrast, we assessed 16 different commercial and noncommercial cultivars as well as pure compounds from these cultivars. Also unlike previous studies that solely evaluated bioactivity, we investigated the mechanism of action mediating bioactivity. We used yeast genetics as a tool to understand the mechanism of action of three compounds (EtOH-vescalagin, estragole and 4-cyclopentene-1,3-dione), which being the first 
time that yeast genetics was used to investigate bioactive compounds in feijoa, was a major contribution of this thesis. As antifungal activity was the focus of this thesis and antifungal mechanism of action is not necessarily conserved across fungal species, future studies will examine the mechanisms of action, identified herein in S. cerevisiae, in Candida and other pathogenic fungi.

For example, the iron chelator ciclopirox is an approved antifungal drug (Sigle, et al. 2005). Given the result from my thesis, it will be interesting to compare the mechanism of action of EtOH-vescalagin and ciclopirox. Ciclopirox inhibits fungal growth by reducing intracellular iron levels. As iron homeostasis is critical to all eukaryotic cells, notably human cells in the case of pathogenic fungi, an antifungal iron chelator is only effective for treating topical fungal infections. Consistent with our results in S. cerevisiae, ciclopirox caused irontransporter upregulation in C. albicans (Sigle, et al. 2005). These results suggest that EtOHvescalagin has potential to be a topical antifungal agent. In order to know if EtOH-vescalagin can offer anything distinct from ciclopirox, we need to compare antifungal mechanism of action for ciclopirox and EtOH-vescalagin. Likewise, the antifungal mechanism of action for 4cyclopentene-1,3-dione that we identified as chitin synthesis inhibition needs to be compared to the established chitin synthesis inhibitors nikkomycin and polyoxin.

My thesis shows that EtOH-vescalagin and 4-cyclopentene-1,3-dione are two of more than 100 antifungal compounds in feijoa. In order to realize pharmaceutical potential, compounds need to meet specific criteria with respect to specificity, potency, and pharmacokinetics. The criteria of specificity may be met for 4-cyclopentene-1,3-dione given its chitin target exists only in fungi and insects. The criteria of potency may also be met for 4cyclopentene-1,3-dione given its $\mathrm{IC}_{50}$ values of $\sim 2 \mu \mathrm{M}$ against pathogenic Candida species. However, the criteria of pharmacokinetics is not clear for 4-cyclopentene-1,3-dione as our results nor any results in literature have examined this compound for pharmacokinetic parameters (e.g., half-life) in an animal model. Less is understood for EtOH-vescalagin. The 
criteria of specificity is not likely met with it being an iron chelator when iron exists in all eukaryotic cells. The criteria of potency may be met for EtOH-vescalagin given its $\mathrm{IC}_{50}$ values of $600 \mu \mathrm{M}$ against pathogenic Candida species. The criteria of pharmacokinetics are not clear for EtOH-vescalagin as our results nor any results in the literature have examined this compound for pharmacokinetic parameters (e.g., half-life) in an animal model.

Drug toxicity has been demonstrated to occur in about one-third of drug candidates (Guengerich 2011) and is particularly critical for antifungal drugs despite efforts to prioritise fungal-specific targets. This toxicity can occur in several different ways such as immune hypersensitivity, off-target toxicity and also covalent modification of the drug. Liver dysfunction caused by antifungal toxicity can lead to elimination of some drugs such as caspofungin and variconazole (Tverdek, et al. 2016). Amphotericin B is a wide spectrum antifungal but its usage has been limited due to side effects and renal toxicity (Veronique Joly 1992). Azole drugs also showed some cell toxicity via interactions with cytochrome P450 complex and deficient cholesterol production (Tverdek, et al. 2016; Veronique Joly 1992) (Marr, et al. 2004). Gastrointestinal, hepatic and endocrinological defects are examples of azole group toxicities. In this project, we introduced EtOH-vescalagin and 4-cyclopentene-1,3-dione as two potential antifungal drugs derived from nature. Both compounds require further investigation at the level of general drug toxicity, and depending on the results, may then proceed in the antifungal drug discovery pipeline.

\subsection{IMPLICATIONS ON HUMAN DISEASES AND THERAPEUTICS}

\subsubsection{Medical mycoses}

Human fungal infections, especially in hospitalized or immunocompromised patients, may have a mortality rate exceeding 50\% despite the availability of well-established antifungal drugs (Badiee and Hashemizadeh 2014). Epistatic drug interactions and resistance are emerging 
as the two main causes of the high mortality seen in fungal infections, which can be alleviated through the discovery of novel antifungal drugs. Fungal infections are treated with compounds targeting fungal cell wall growth or general eukaryotic growth (Denning and Bromley 2015). In this thesis, the antifungal activities of EtOH-vescalagin (via iron chelation, zinc toxicity and retromer dysfunction), estragole (a novel mechanism) and 4-cyclopentene-1,3-dione (via chitin synthesis inhibition) were reported from feijoa fruit.

We have shown EtOH-vescalagin is antifungal through an iron chelation mechanism. Iron chelation as a drug target has been investigated to treat a range of diseases, of which fungal diseases has recently joined that list of diseases. The first orally available iron chelator (deferioprone) was approved in 2005 to treat thalassaemia (Olabinri, et al. 2010) (Ibrahim, et al. 2008), and this drug has recently been investigated for antifungal activity against Aspergillus fumigatus (Zarember, et al. 2009). There are mixed results to date for antifungal activity of iron chelators. The iron chelators ciclopirox and deferiprone have shown promise as antifungal agents, while deferoxamine has not shown promise (Zarember, et al. 2009). The combination therapy of deferiprone or ciclopirox with antifungal drugs that inhibit ergosterol synthesis showed potent antifungal activity (Zarember, et al. 2009). Likewise, deferoxamine increased the amphotericin B antifungal activity against Cryptococcus species (Lai, et al. 2016). Mucormycoses in immunocompromised patients caused by zygomycete fungi are susceptible to a range of iron chelators such as deferasirox (Ibrahim, et al. 2008). Altogether, the future is bright for iron chelation as a mechanism to treat medical mycoses.

Zinc toxicity was the second mechanism of EtOH-vescalagin demonstrated in this thesis. Metals are needed for normal cell function but an excess amount of each can cause metal toxicity and cell death (Soares, et al. 2003). It was shown that some cellular defects in DNA damage, protein synthesis, lipid biosynthesis and oxidative stress can lead to metal toxicity (Hosiner, et al. 2014). Zinc toxicity is not well studied as a mechanism of antifungal therapy. 
Thus, zinc toxicity as an antifungal mechanism needs more studies to confirm its potential for being approved as an antifungal agent.

Retromer dysfunction was the third antifungal mechanism of EtOH-vescalagin in $S$. cerevisiae as a secondary mechanism of antifungal activity. The retromer complex is required for several critical processes including signaling, protein transport and lipid transport (Bowers and Stevens 2005). Despite being a target to treat neurodegenerative diseases (Small and Petsko 2015), retromer has not been targeted to treat fungal infections. However, there is some compelling information in addition to this thesis that warrants further investigation of retromer as an antifungal target. Growth of the fungal plant pathogen Fusarium graminearum was impeded with retromer dysfunction (Zheng, et al. 2016). Retromer recycling is also essential for virulence of Candida albicans in mice (Liu, et al. 2014). Altogether, the retromer complex has not been specifically targeted to treat fungal infections, but there is compelling evidence to pursue retromer function as an antifungal target.

Estragole was shown to be antifungal against pathogenic Candida species in this thesis, and this occurred via a mechanism not currently targeted by antifungal drugs. Several studies prior to the start of this thesis reported antifungal activity of estragole against pathogenic fungi (Waller, et al. 2017); however, these studies did not report an antifungal mechanism for estragole except for a commonly identified mechanism of increased oxidative stress (Khan, et al. 2011). Estragole on its own does not have the potency to be considered for pharmaceutical potential with $\mathrm{IC}_{50}$ values against Candida species $\sim 177-233 \mu \mathrm{M}$, a concentration that is dramatically more than the $10 \mu \mathrm{M}$ required for a pharmaceutical drug. However, combinatorial treatments of estragole with ketoconazole and amphotericin B have shown increased antifungal activity compared to the single treatments alone (Shin and Kang 2003; Shin and Pyun 2004). With some promise to be included in a dual treatment with existing antifungals, it would be 
informative to determine the antifungal mechanism of action of estragole to fully understand the two pathways targeted in the dual treatment.

\subsubsection{Cancer}

The prevalence of cancer is continuing to grow, with annual rates of new cases expected to increase to 20 million by 2025 worldwide (Ferlay, et al. 2015). There were an estimated 14.1 million new cases of cancer and 8.2 million cancer related deaths in 2012 alone. Whilst there are several therapies available to treat an array of cancers, none of them are able to cure the disease completely (Prakash, et al. 2013). Therefore, the search for new cancer therapies is as active as ever. Natural products offer a rich source of bioactive compounds as a potential source for novel cancer therapeutics.

Chitin synthase inhibitors have been shown to have anti-cancer activity targeting chitin-like molecules such as glycan (oligosaccharides) as these molecules are different in cancer cells in structure and abundance compared to normal cells (Dube and Bertozzi 2005; Wattenberg, et al. 2010). Due to the ability of 4-cyclopentene-1,3-dione to inhibit chitin synthesis in yeast, we hypothesized that 4-cyclopentene-1,3-dione would have anti-cancer activity. An MTT assay is a colorimetric assay measurement of $\mathrm{NAD}(\mathrm{P}) \mathrm{H}$-dependent cellular oxidoreductase that is representative of viability and a reliable starting point to evaluating anticancer activity (Hood, et al. 2001). Preliminary data collected in collaboration with Prof John Miller (Victoria University of Wellington) indicated that 4-cyclopentene-1,3-dione had anticancer activity in HL60 leukemia cells with an $\mathrm{IC}_{50}$ of $1.6 \mu \mathrm{M}$, which is comparable to the $\mathrm{IC}_{50}$ of $1.3 \mu \mathrm{M}$ in yeast (Figure 5.2A). However, more work is required to elucidate the anti-cancer activity of 4-cyclopentene-1,3-dione, such as using additional cancer cancer cell lines as well as control cell lines to distinguish general toxicity in mammalian cells from cancer-specific toxicity. If there is not general toxicity, it will be interesting to investigate the anti-cancer mechanisms already reported for chitin synthesis inhibitors (Dube and Bertozzi 2005). 
A

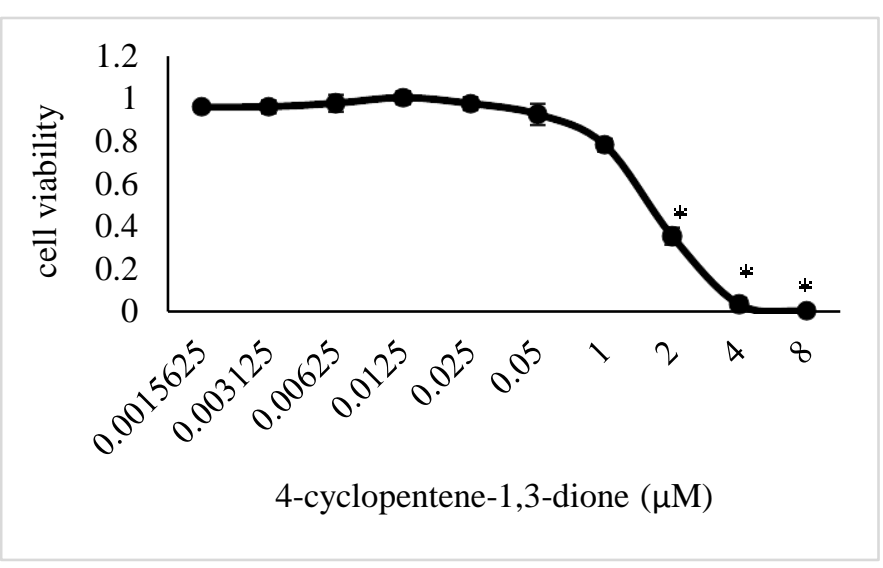

B

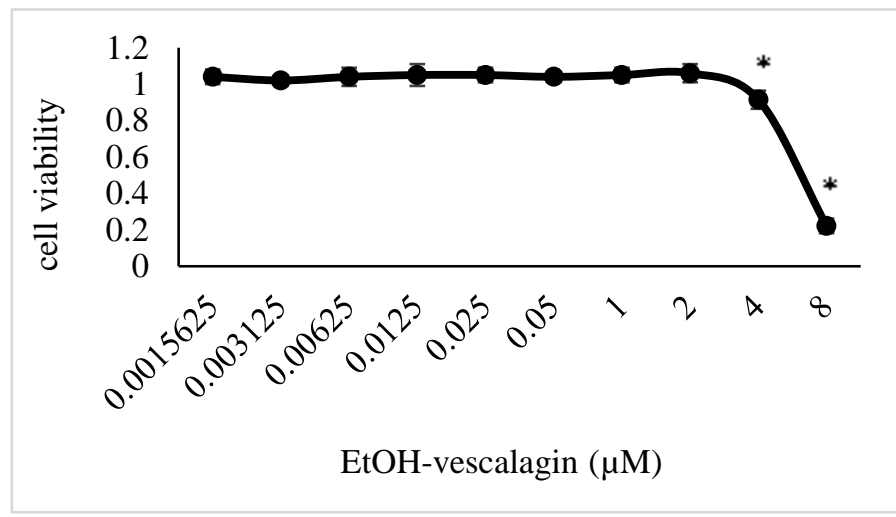

Figure 5-1. Preliminary data suggest anti-cancer activity of two feijoa compounds. Viability of HL60 cells was measured using MTT assay in A) 4-Cyclopentene-1,3-dione and B) EtOH-vescalagin anticancer activity in HL60 cells. *, $p<0.05$, Student's $t$-test comparison with untreated.

We also tested the anti-cancer potential of EtOH-vescalagin, which showed anticancer activity with an $\mathrm{IC}_{50}$ of $5.7 \mu \mathrm{M}$ (Figure 5.1B). This result conveys that EtOH-vescalagin is more potent as an antifungal agent $\left(\mathrm{IC}_{50} \sim 600 \mathrm{nM}\right)$ than an anti-cancer agent. However, this difference should be loosely interpreted since the results are dependent on cell lines being tested, that often requires more drug for toxicity than other cancer cell lines (Waghorne, et al. 2016).

In HL60 as well as additional cancer cell lines, it will be interesting to determine if the antifungal mechanisms of EtOH-vescalagin (iron chelation, zinc toxicity and retromer 
dysfunction) are also anti-cancer mechanisms of EtOH-vescalagin. As iron deficiency causes oxidative stress in cancer cells leading to cell death (Buss, et al. 2003), several iron chelators have exhibited promising anti-cancer activity such as tachpyridine, desferrithiocin, O-trensox and deferasirox (Bedford, et al. 2013; Buss, et al. 2003; Lui, et al. 2013). Likewise, inducing zinc toxicity has promise since some cancers (e.g., ovarian, prostate, pancreatic) have reduced zinc levels to avoid anti-tumor suppressor effects (Costello and Franklin 2012) (Costello, et al. 2011) (Franklin, et al. 2005) (Lightman, et al. 1986). In contrast to iron and zinc homeostasis, the role for retromer in cancer is not well understood. Thus far it has been shown that retromer somehow regulates the sensitivity of cancer cells to anti-cancer drugs (Oqi et al 2013), a result that would be consistent with our findings that retromer dysfunction exacerbated $\mathrm{EtOH}-$ vescalagin sensitivity in yeast cells. In summary, the mechanisms of EtOH-vescalagin (iron chelation, zinc toxicity, retromer dysfunction) are all candidate mechanisms to pursue in an anti-cancer agent, which suggests EtOH-vescalagin is worth further investigation as an anticancer agent.

\subsubsection{Diabetes}

Diabetes mellitus type 2 , herein referred to as type 2 diabetes, is the sixth highest cause of death in US accounting for more than 73,000 deaths annually (Ripsin, et al. 2009) and affecting at least 257,000 New Zealanders (Ministry of Health, New Zealand Report, 2015). Type 2 diabetes is caused by insulin resistance, reduced insulin secretion and finally high blood sugar which can cause kidney function failure and blindness in adults predominately due to high fat conditions in obesity (Kahn, et al. 2006). Management of diabetes focuses on lifestyle management as well as controlling blood sugar levels and fat levels (Tuomilehto, et al. 2001).

The implications of EtOH-vescalagin on diabetes are dependent on the functional consequences of targeting the mechanisms of EtOH-vescalagin (iron chelation, zinc toxicity, retromer dysfunction). Increased heme iron has long been associated with diabetes type 2 risk 
(Wrede, et al. 2006) and iron toxicity has been reported to cause insulin deficiency. In addition, iron chelators ameliorated diabetes in mice (Cooksey, et al. 2010). Zinc deficiency also contributes to diabetes type 2 , which was the rationale for zinc supplementation studies that were beneficial in diabetic humans controlling glucose metabolism and lipid homeostasis (Jayawardena, et al. 2012). Likewise, retromer has recently been shown to be critical in the regulation of glucose metabolism and lipid homeostasis in diabetes (Yang et al 2016; Pan et al 2017). We were thus prompted to investigate the anti-diabetic potential of EtOH-vescalagin.

Our preliminary data on the effect of EtOH-vescalagin on glucose metabolism and lipotoxicity (a metabolic state associated with obesity and diabetes) are potentially interesting. Given our promising anti-diabetes results with EtOH-vescalagin, it will be interesting to examine other ellagitannin compounds in feijoa fruit, such as the compounds in feijoa extracts that have been shown to exhibit anti-diabetic effects in human patients and rodents (Foo and Watson 2013).

Glucose is the primary preference of a cell as a carbon source to produce energy (Kayikci and Nielsen 2015). SNF1 is a conserved yeast serine/threonine kinase that is required for glucose metabolism via AMPK in mammals, the target of the popular anti-diabetic drug metformin (Fryer, et al. 2002). To initially assess if there is an association between SNF1 and EtOH-vescalagin, we quantified growth of $s n f 1 \Delta$ in the presence and absence of $305 \mathrm{nM} \mathrm{EtOH}$ vescalagin. This treatment resulted in significant growth reduction of $\operatorname{snf} 1 \Delta$ by $52 \%$ compared to untreated cells, a result in contrast to the $20 \%$ inhibition in WT compared to untreated cells (Figure 5.3A). To further investigate the effect of EtOH-vescalagin on glucose metabolism, we quantified growth of WT yeast in varying amounts of glucose $(2,4,8 \%)$ in the presence and absence of EtOH-vescalagin, the zinc chelator, TPEN or estragole. The increasing glucose concentrations resulted in significantly less growth in a dose-dependent manner in the TPEN 
and estragole treatments, while the $\mathrm{EtOH}$-vescalagin resulted in significanty more growth with increasing glucose concentrations (Figure 5.3B).

A

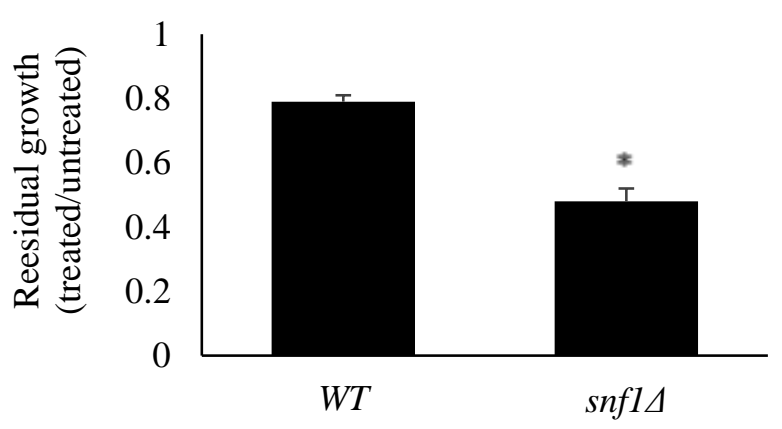

B

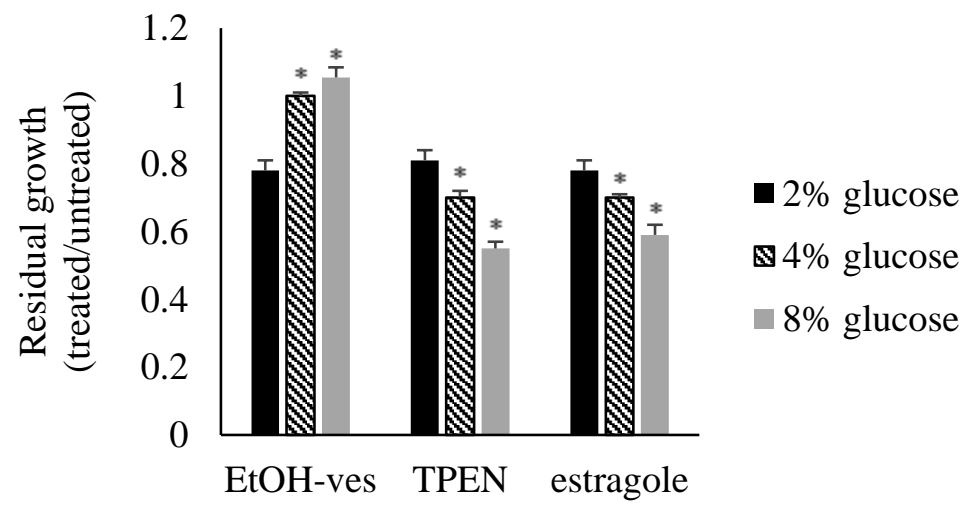

Figure 5-2. Preliminary data suggests EtOH-vescalagin regulates glucose metabolism homeostasis. A) Haploid cells were grown to mid-log in triplicate and growth was measured at 14 hours. B) Cells were grown to mid-log in various glucose concentrations. $90 \mu \mathrm{M}$ estragole and $1 \mu \mathrm{M}$ TPEN were used as control drugs to compare to EtOH-vescalagin. ${ }^{*}, p<0.05$, Student's $t$-test comparison with relative control.

Almost all eukaryotes synthesise lipid droplets (LD) that are used for the storage of neutral lipids, such as triacylglycerol (TAG) and sterols (Choudhary, et al. 2011). Inducing high levels of LDs in cells has been used to mimic the cellular phenotype of diabetes (Zlobine, et al. 2016). We hypothesized that a consequence of EtOH-vescalagin treatment may be reduction in the size or numbers of lipid droplets. We thus treated WT strains as well as $\operatorname{tgl} 3 \triangle$ and $\operatorname{tgl} 4 \Delta$ (established yeast models of obesity with significantly high numbers of lipid droplets) (Kurat, 
et al. 2006) and visualized lipid droplets using Nile Red, a fluorescent stain for lipid droplets (Sitepu, et al. 2012). The EtOH-vescalagin treatment decreased lipid droplet production (size and abundance) in WT, $\operatorname{tgl} 3 \Delta$ and $\operatorname{tg} l 4 \Delta$ by 22,41 and $35 \%$, respectively, compared to untreated cells (Figure 5.4).

A

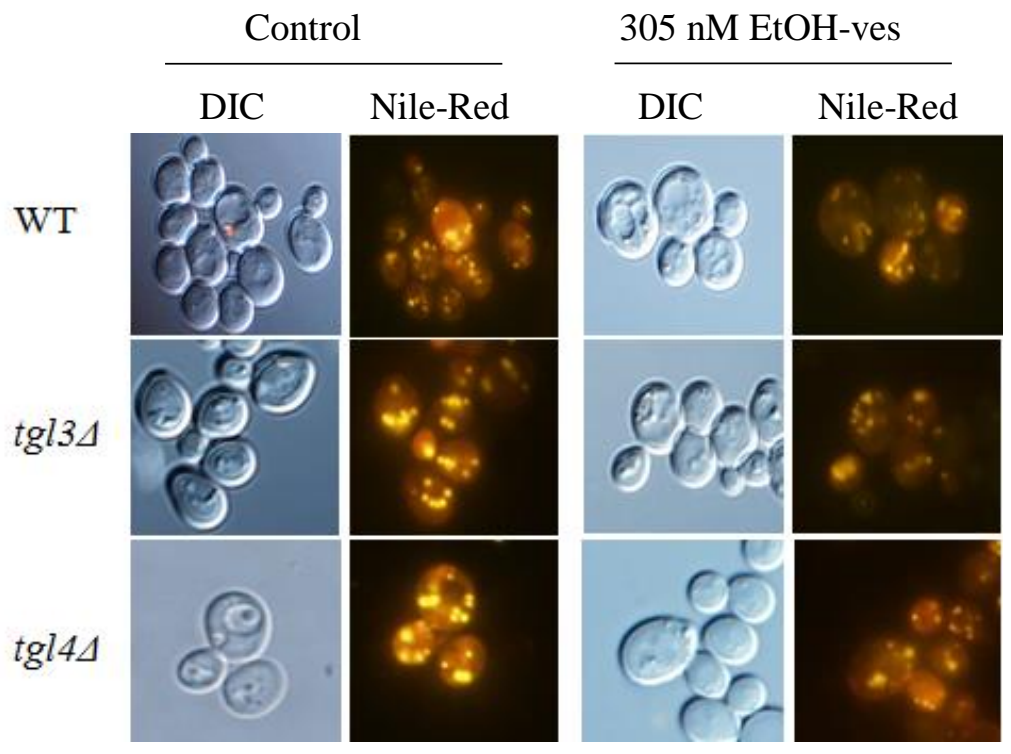

B

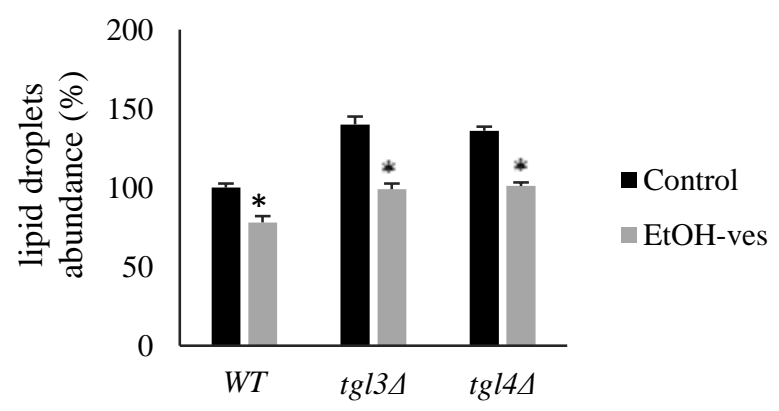

Figure 5-3. Preliminary data suggest EtOH-vescalagin regulates lipid droplets production. A) Cells were grown and stained at mid-log phase with Nile Red for $20 \mathrm{~min}$ and visualized under Olympus fluorescent microscope by GFP filter. B) Image analysis was done by Fiji-imagej and values are mean \pm SD. *,$p<0.05$, Student's $t$-test comparison with relative control.

Proteins are produced in the ER and subsequently folded, modified and transferred to other organelles in eukaryotic cells. ER homeostasis is disrupted in diabetes, which induces the unfolded protein respone (UPR) leading to pancreatic $\beta$-cell death (Back and Kaufman 2012; 
Malhotra and Kaufman 2011). To determine if EtOH-vescalagin can reduce the UPR, we measured the UPR via a fluorescent reporter comprised of four elements of the UPR fused to a GFP (Jonikas, et al. 2009). Fluorescence was visualized and quantified in WT as well as the dgalAlro1/ strain that is deficient at producing lipid droplets and sensitive to UPR (Choudhary, et al. 2011). The UPR was induced in both strains (with tunicamycin in WT or with the fatty

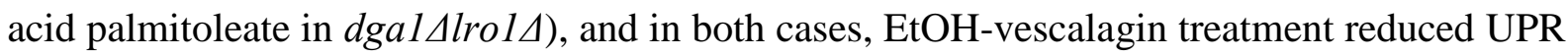
to a similar extent as zinc supplementation (Figure 5.5A). 
A)

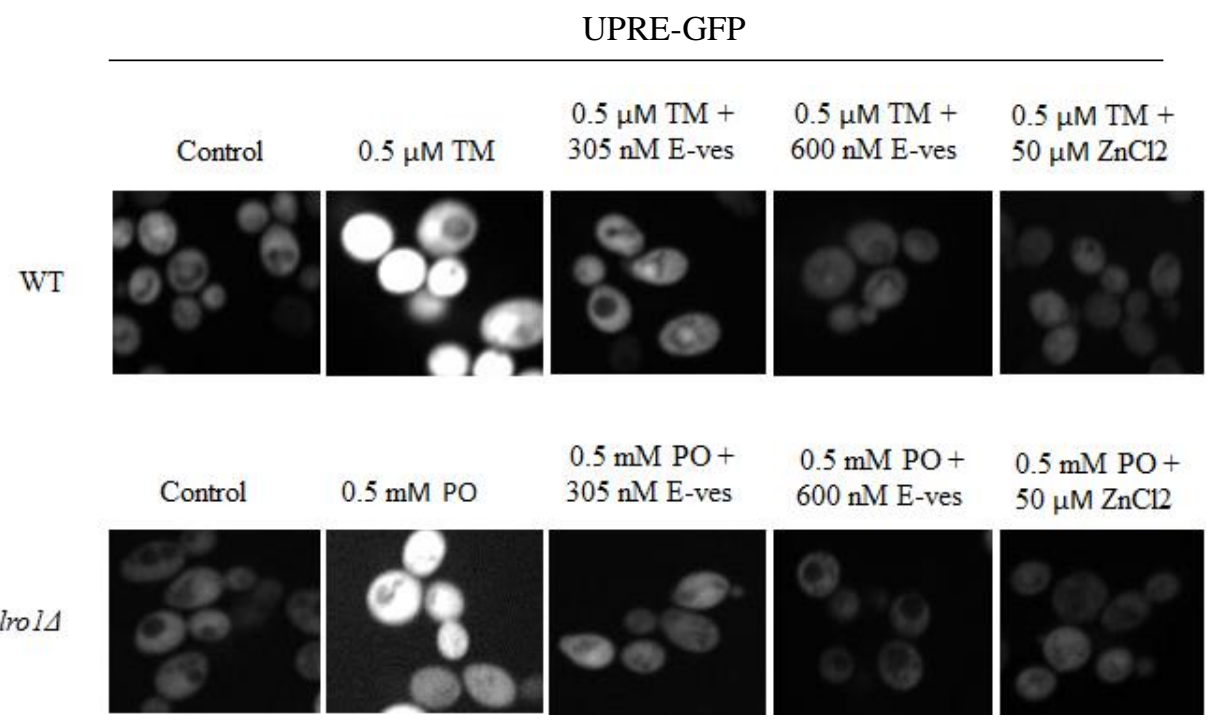

B)

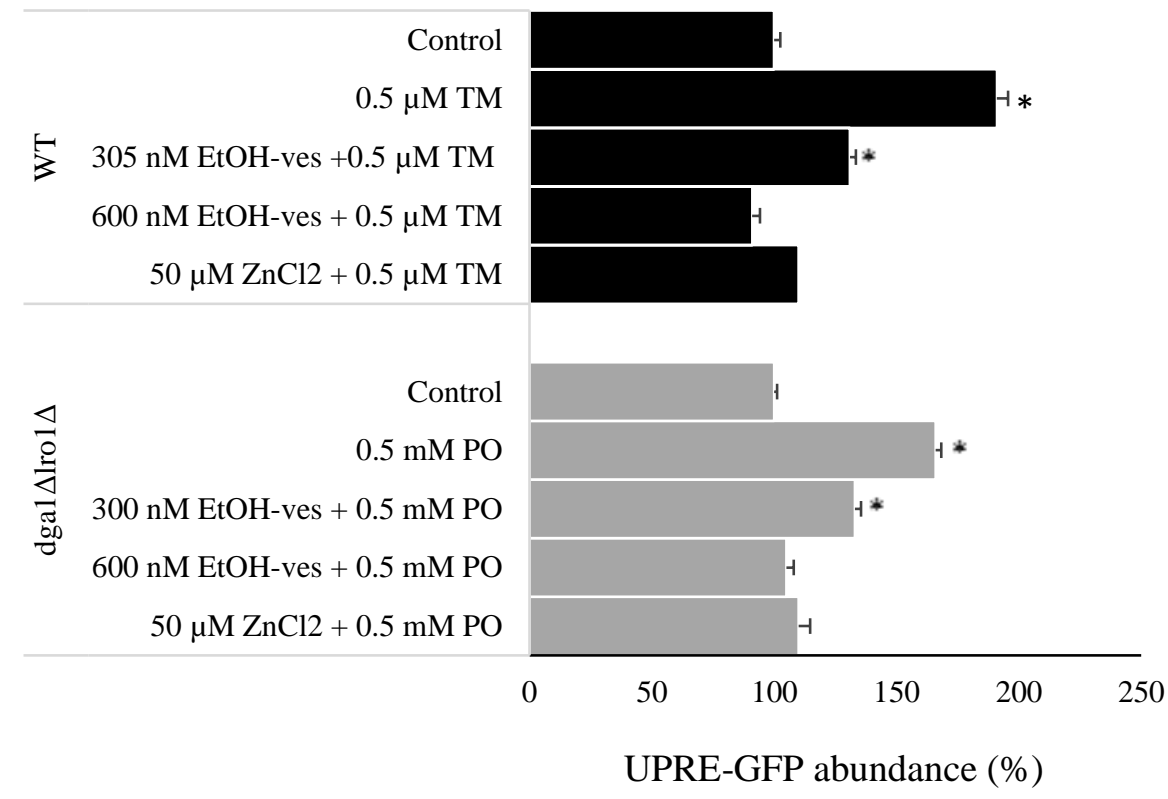

Figure 5-4. Preliminary data suggests EtOH-vescalagin regulates unfolded protein response. A) Cells expressing UPRE-GFP were grown and treated with tunicamycin (TM) for 4 hours and then treated with EtOH-vescalagin (EtOH-ves) for 4 hours. $\mathrm{ZnCl}_{2}$ was used as a control to decrease UPRE.

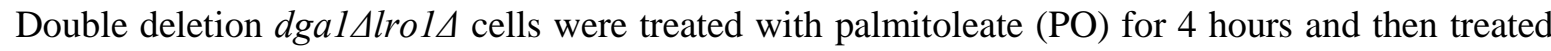
with EtOH-vescalagin for 4 hours. Images were taken by Opera microscope (Perkin Elmer). B) Quantification of UPRE level using Fiji-imagej. *, $p<0.05$, Student's $t$-test comparison with relative control. UPRE: unfolded protein response. 
These results are consistent with EtOH-vescalagin reducing UPR via increased intracellular levels of zinc (Piao, et al. 2016; Xu, et al. 2011a). In WT, tunicamycin treatment caused 91\% UPRE increase while after EtOH-vescalagin treatment with 305 and $600 \mathrm{nM}$ it was

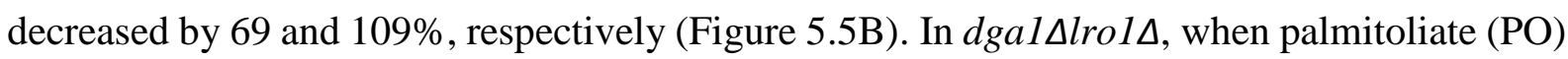
treatment caused 66\% UPRE increase, 305 and $600 \mathrm{nM}$ EtOH-vescalagin decreased UPRE level by 30 and $61 \%$, respectively.

\subsection{IMPLICATION ON AGRICULTURE}

Compounds studied in this thesis are natural compounds that have been functional in nature for millions of years or at least since the domestication of crops, in this case feijoa. The compounds thus likely have importance in the agricultural setting. This brings up the question of whether these antifungal compounds can be used as antifungal agents in an agricultural setting. The main mechanism of EtOH-vescalagin, iron chelation, has been targeted by the antifungal compound lactoferrin to combat plant pathogens such as Botrytis cinerea, Sclerotinium rolsfi, Rhizoctonia solani and Fusarium oxysporum (Fernandes and Carter 2017). In contrast, the zinc toxicity and retromer dysfunction mechanisms of EtOH-vescalagin have not been investigated as antifungal targets in an agricultural setting. There is thus potential to investigate the EtOH-vescalagin as an antifungal agent in agriculture. Likewise, there is potential for the chitin synthesis inhibitor 4-cyclopentene-1,3-dione as a dual antifungal and insecticide. A compound of commercial use isolated from feijoa peels would add value to feijoa as a crop since the source of these compounds (the peel) is a waste product (e.g., peels are completely disposed when feijoa juice is produced). 


\subsection{CONCLUSION}

To conclude, this project elucidated the antifungal mechanism of action for compounds derived from feijoa fruit using chemical genetics and genomics. Metabolite profiling of 16 feijoa cultivars distinguished two potent cultivars as well as 4-cyclopentene-1,3-dione with potent antifungal activity. These results emphasize that subtle genetic differences between cultivars comprise major chemical differences in bioactive compounds. We identified that the EtOH-vescalagin is an iron chelator that disrupts zinc homeostasis and retromer recycling. This result revealed for the first time an antifungal mechanism that links iron, zinc and retromer. We also determined that the antifungal mechanism of estragole is distinct from known antifungals, which may represent a new class of antifungals. Together, the results in this thesis contributed to the genetic and chemical understanding of feijoa, and moreover, identified key compounds and mechanisms in feijoa compounds that have pharmaceutical potential to treat fungal infections, cancer and diabetes. 



\section{References}

Achterman RR, White TC 2011. Dermatophyte virulence factors: identifying and analyzing genes that may contribute to chronic or acute skin infections. International journal of microbiology 2012.

Adlercreutz H, Mazur W 1997. Phyto-oestrogens and Western diseases. Annals of medicine 29: 95-120.

Alberto Moldes C, Fontão de Lima Filho O, Manuel Camiña J, Gabriela Kiriachek S, Lia Molas Ma, Mui Tsai S 2013. Assessment of the effect of silicon on antioxidant enzymes in cotton plants by multivariate analysis. Journal of agricultural and food chemistry 61: 11243-11249.

Amaral S, Mira L, Nogueira J, da Silva AP, Florêncio MH 2009. Plant extracts with anti-inflammatory properties-A new approach for characterization of their bioactive compounds and establishment of structure-antioxidant activity relationships. Bioorganic \& medicinal chemistry 17: 1876-1883.

Ammar R, Smith AM, Heisler LE, Giaever G, Nislow C 2009. A comparative analysis of DNA barcode microarray feature size. BMC genomics 10: 1.

Amrouche A, Benmehdi H, Moussaoui A, Mebarki K, Chaoufi A, Saneba A, Lazouni H, Sari DC 2011. Evaluation of antifungal activity of some oils from Algerian medicinal plants against Aspergillus flavus strain produced aflatoxins.

Andrade TC, LIMA SG, Freitas RM, Rocha MS, Islam T, SILVA TG, Militao GC 2015. Isolation, characterization and evaluation of antimicrobial and cytotoxic activity of estragole, obtained from the essential oil of croton zehntneri (euphorbiaceae). Anais da Academia Brasileira de Ciências 87: 173182.

Andreyev AY, Kushnareva YE, Starkov A 2005. Mitochondrial metabolism of reactive oxygen species. Biochemistry (Moscow) 70: 200-214.

Andriole VT 2000. Current and future antifungal therapy: new targets for antifungal therapy. International journal of antimicrobial agents 16: 317-321.

Arif T, Bhosale J, Kumar N, Mandal T, Bendre R, Lavekar G, Dabur R 2009. Natural productsantifungal agents derived from plants. Journal of Asian Natural Products Research 11: 621-638.

Arlt H, Reggiori F, Ungermann C 2015. Retromer and the dynamin Vps1 cooperate in the retrieval of transmembrane proteins from vacuoles. J Cell Sci 128: 645-655.

Arthington-Skaggs B, Crowell D, Yang H, Sturley S, Bard M 1996. Positive and negative regulation of a sterol biosynthetic gene (ERG3) in the post-squalene portion of the yeast ergosterol pathway. FEBS Lett 392: 161-165.

Ashburner M, Ball CA, Blake JA, Botstein D, Butler H, Cherry JM, Davis AP, Dolinski K, Dwight SS, Eppig JT 2000. Gene Ontology: tool for the unification of biology. Nature genetics 25: 25-29.

Ashurst PR. 2012. Food flavorings: Springer Science \& Business Media.

Assing K, Birgens H, Arendrup M 2003. Cryptococcus neoformans var. neoformans resistant to fluconazole in an HIV-negative patient with chronic lymphocytic leukemia. Clinical Microbiology and Infection 9: 441-444. doi: https://doi.org/10.1046/j.1469-0691.2003.00571.x 
Atanasov AG, Waltenberger B, Pferschy-Wenzig E-M, Linder T, Wawrosch C, Uhrin P, Temml V, Wang L, Schwaiger S, Heiss EH 2015. Discovery and resupply of pharmacologically active plantderived natural products: A review. Biotechnology advances 33: 1582-1614.

Auzanneau C, Montaudon D, Puyo S, Jacquet R, Elkaoukabi-Chaibi A, Quideau S, De Giorgi F, Ichas F, Pourquier P. 2011. Vescalagin, a polyphenolic ellagitanin specifically inhibiting the alpha isoform of human DNA topoisomerase II. In: AACR.

Back SH, Kaufman RJ 2012. Endoplasmic reticulum stress and type 2 diabetes. Annual review of biochemistry 81: 767-793.

Badiee P, Hashemizadeh Z 2014. Opportunistic invasive fungal infections: diagnosis \& clinical management. Indian Journal of Medical Research 139: 195.

Balakrishnan R, Park J, Karra K, Hitz BC, Binkley G, Hong EL, Sullivan J, Micklem G, Michael Cherry J 2012. YeastMine - an integrated data warehouse for Saccharomyces cerevisiae data as a multipurpose tool-kit. Database 2012.

Balandrin MF, Kinghorn AD, Farnsworth NR editors. ACS symposium series (USA). 1993.

Barbosa Pelegrini P, del Sarto RP, Silva ON, Franco OL, Grossi-de-Sa MF 2011. Antibacterial peptides from plants: what they are and how they probably work. Biochemistry Research International 2011.

Basile A, Conte B, Rigano D, Senatore F, Sorbo S 2010. Antibacterial and antifungal properties of acetonic extract of Feijoa sellowiana fruits and its effect on Helicobacter pylori growth. Journal of medicinal food 13: 189-195.

Basile A, Vuotto M, Violante U, Sorbo S, Martone G, Castaldo-Cobianchi R 1997. Antibacterial activity in Actinidia chinensis, Feijoa sellowiana and Aberia caffra. Int J Antim Ag 8: 199-203.

Becker H, Scher JM, Speakman J-B, Zapp J 2005. Bioactivity guided isolation of antimicrobial compounds from Lythrum salicaria. Fitoterapia 76: 580-584.

Bedford MR, Ford SJ, Horniblow RD, Iqbal TH, Tselepis C 2013. Iron chelation in the treatment of cancer: a new role for deferasirox? The Journal of Clinical Pharmacology 53: 885-891.

Ben-Aroya S, Pan X, Boeke JD, Hieter P 2010. Making temperature-sensitive mutants. Methods in enzymology 470: 181-204.

Bender A, Pringle JR 1991. Use of a screen for synthetic lethal and multicopy suppressee mutants to identify two new genes involved in morphogenesis in Saccharomyces cerevisiae. Molecular and Cellular Biology 11: 1295-1305.

Berger KH, Sogo LF, Yaffe MP 1997. Mdm12p, a component required for mitochondrial inheritance that is conserved between budding and fission yeast. The Journal of cell biology 136: 545-553.

Berry-Kilgour C 2002. Pseudowintera colorata: a unique New Zealand medicinal herb. Australian Journal of Medical Herbalism 14: 67.

Best HA, Matthews JH, Heathcott RW, Hanna R, Leahy DC, Coorey NV, Bellows DS, Atkinson PH, Miller JH 2013. Laulimalide and peloruside A inhibit mitosis of Saccharomyces cerevisiae by preventing microtubule depolymerisation-dependent steps in chromosome separation and nuclear positioning. Molecular BioSystems 9: 2842-2852. 
Bhakuni DS, Rawat DS. 2006. Bioactive marine natural products: Springer Science \& Business Media. Binder RG, Flath RA 1989. Volatile components of pineapple guava. J Agric Food Chem 37: 734-736.

Birben E, Sahiner UM, Sackesen C, Erzurum S, Kalayci O 2012. Oxidative stress and antioxidant defense. World Allergy Organization Journal 5: 9.

Bircham PW, Maass DR, Roberts CA, Kiew PY, Low YS, Yegambaram M, Matthews J, Jack CA, Atkinson PH 2011. Secretory pathway genes assessed by high-throughput microscopy and synthetic genetic array analysis. Molecular BioSystems 7: 2589-2598.

Blasiak J, Glowacki S, Kauppinen A, Kaarniranta K 2013. Mitochondrial and nuclear DNA damage and repair in age-related macular degeneration. International journal of molecular sciences 14: 2996-3010.

Bleackley MR, MacGillivray RT 2011. Transition metal homeostasis: from yeast to human disease. Biometals 24: 785-809.

Blomgren J, Berggren U, Jontell M 1998. Fluconazole versus nystatin in the treatment of oral candidosis. Acta Odontologica Scandinavica 56: 202-205.

Bondaryk M, Kurzątkowski W, Staniszewska M 2013. Antifungal agents commonly used in the superficial and mucosal candidiasis treatment: mode of action and resistance development. Postepy Dermatol Alergol 30: 293-301.

Bontempo P, Mita L, Miceli M, Doto A, Nebbioso A, De Bellis F, Conte M, Minichiello A, Manzo F, Carafa V 2007. Feijoa sellowiana derived natural Flavone exerts anti-cancer action displaying HDAC inhibitory activities. Int J Biochem Celll Biol 39: 1902-1914.

Boone C, Bussey H, Andrews BJ 2007. Exploring genetic interactions and networks with yeast. Nature Reviews Genetics 8: 437-449.

Botstein D, Fink GR 2011. Yeast: an experimental organism for 21st Century biology. Genetics 189: 695-704.

Bowers K, Stevens TH 2005. Protein transport from the late Golgi to the vacuole in the yeast Saccharomyces cerevisiae. Biochimica et Biophysica Acta (BBA)-Molecular Cell Research 1744: 438454.

Breker M, Gymrek M, Schuldiner M 2013. A novel single-cell screening platform reveals proteome plasticity during yeast stress responses. J Cell Biol 200: 839-850.

Breslow DK, Cameron DM, Collins SR, Schuldiner M, Stewart-Ornstein J, Newman HW, Braun S, Madhani HD, Krogan NJ, Weissman JS 2008. A comprehensive strategy enabling high-resolution functional analysis of the yeast genome. Nature methods 5: 711-718.

Brown GD, Denning DW, Gow NAR, Levitz SM, Netea MG, White TC 2012. Hidden Killers: Human Fungal Infections. Science Translational Medicine 4: 165rv113-165rv113. doi: 10.1126/scitranslmed.3004404

Burda P, Padilla SM, Sarkar S, Emr SD 2002. Retromer function in endosome-to-Golgi retrograde transport is regulated by the yeast Vps34 PtdIns 3-kinase. Journal of cell science 115: 3889-3900.

Buss JL, Torti FM, Torti SV 2003. The role of iron chelation in cancer therapy. Current medicinal chemistry 10: 1021-1034. 
Butler MS 2004a. The role of natural product chemistry in drug discovery. Journal of Natural Products 67: 2141-2153.

Butler MS 2004b. The role of natural product chemistry in drug discovery. J Nat Prod 67: 2141-2153.

Cabib E 1991. Differential inhibition of chitin synthetases 1 and 2 from Saccharomyces cerevisiae by polyoxin D and nikkomycins. Antimicrobial agents and chemotherapy 35: 170-173.

Caffrey P, Lynch S, Flood E, Finnan S, Oliynyk M 2001. Amphotericin biosynthesis in Streptomyces nodosus: deductions from analysis of polyketide synthase and late genes. Chemistry \& biology 8: 713723.

Camadro J-M, Labbe P 1988. Purification and properties of ferrochelatase from the yeast Saccharomyces cerevisiae. Evidence for a precursor form of the protein. Journal of Biological Chemistry 263: $11675-11682$.

Campoy S, Adrio JL 2017. Antifungals. Biochemical Pharmacology. doi: http://dx.doi.org/10.1016/j.bcp.2016.11.019

Chakrabarti A, Shivaprakash M 2005. Microbiology of systemic fungal infections. Journal of postgraduate medicine 51: 16 .

Chen JK, Lane WS, Schreiber SL 1999. The identification of myriocin-binding proteins. Chemistry \& biology 6: 221-235.

Choudhary V, Jacquier N, Schneiter R 2011. The topology of the triacylglycerol synthesizing enzyme Lro1 indicates that neutral lipids can be produced within the luminal compartment of the endoplasmatic reticulum: Implications for the biogenesis of lipid droplets. Communicative \& integrative biology 4 : 781-784.

Chu T, Sun J, Saksena S, Emr SD 2006. New component of ESCRT-I regulates endosomal sorting complex assembly. J Cell Biol 175: 815-823.

Cooksey RC, Jones D, Gabrielsen S, Huang J, Simcox JA, Luo B, Soesanto Y, Rienhoff H, Abel ED, McClain DA 2010. Dietary iron restriction or iron chelation protects from diabetes and loss of $\beta$-cell function in the obese (ob/ob lep-/-) mouse. American Journal of Physiology-Endocrinology and Metabolism 298: E1236-E1243.

Cooper AA, Gitler AD, Cashikar A, Haynes CM, Hill KJ, Bhullar B, Liu K, Xu K, Strathearn KE, Liu F 2006. $\alpha$-Synuclein blocks ER-Golgi traffic and Rab1 rescues neuron loss in Parkinson's models. Science 313: 324-328.

Costa-de-Oliveira S, Silva AP, Miranda IM, Salvador A, Azevedo MM, Munro CA, Rodrigues AG, Pina-Vaz C 2013. Determination of chitin content in fungal cell wall: an alternative flow cytometric method. Cytometry Part A 83: 324-328.

Costanzo M, VanderSluis B, Koch EN, Baryshnikova A, Pons C, Tan G, Wang W, Usaj M, Hanchard J, Lee SD 2016. A global genetic interaction network maps a wiring diagram of cellular function. Science 353: aaf1420.

Costello LC, Franklin RB 2012. Cytotoxic/tumor suppressor role of zinc for the treatment of cancer: an enigma and an opportunity. Expert review of anticancer therapy 12: 121-128. 
Costello LC, Levy BA, Desouki MM, Zou J, Bagasra O, Johnson LA, Hanna N, Franklin RB 2011. Decreased zinc and downregulation of ZIP3 zinc uptake transporter in the development of pancreatic adenocarcinoma. Cancer biology \& therapy 12: 297-303.

Courel M, Lallet S, Camadro J-M, Blaiseau P-L 2005. Direct activation of genes involved in intracellular iron use by the yeast iron-responsive transcription factor Aft 2 without its paralog Aft1. Molecular and Cellular Biology 25: 6760-6771.

Coutinho ID, Baker JM, Ward JL, Beale MH, Creste S, Cavalheiro AJ 2016. Metabolite profiling of sugarcane genotypes and identification of flavonoid glycosides and phenolic acids. Journal of agricultural and food chemistry 64: 4198-4206.

Cowan MM 1999. Plant products as antimicrobial agents. Clinical microbiology reviews 12: 564-582.

Cragg GM, Newman DJ 2013. Natural products: a continuing source of novel drug leads. Biochimica et Biophysica Acta (BBA)-General Subjects 1830: 3670-3695.

Crofts TS, Gasparrini AJ, Dantas G 2017. Next-generation approaches to understand and combat the antibiotic resistome. Nature Reviews Microbiology.

Curator PS 2004. NEW ZEALAND REFERENCE CULTURE, COLLECTION, MEDICAL SECTION, CATALOGUE OF STRAINS.

Cyert MS, Philpott CC 2013. Regulation of cation balance in Saccharomyces cerevisiae. Genetics 193: 677-713.

Daferner M, Mensch S, Anke T, Sterner O 1999. Hypoxysordarin, a new sordarin derivative from Hypoxylon croceum. Zeitschrift für Naturforschung C 54: 474-480.

Daglia M 2012. Polyphenols as antimicrobial agents. Current opinion in biotechnology 23: 174-181.

Dalleau S, Cateau E, Bergès T, Berjeaud J, limbert C 2007. P629 In vitro activity of essential oils and their major components against Candida albicans yeasts growing planktonically and as biofilms. International journal of antimicrobial agents 29: S147.

Damen E, Krieger E, Nielsen JE, Eygensteyn J, Van Leeuwen JE 2006. The human Vps29 retromer component is a metallo-phosphoesterase for a cation-independent mannose 6-phosphate receptor substrate peptide. Biochemical Journal 398: 399-409.

de Souza LM, Cipriani TR, Iacomini M, Gorin PA, Sassaki GL 2008. HPLC/ESI-MS and NMR analysis of flavonoids and tannins in bioactive extract from leaves of Maytenus ilicifolia. Journal of pharmaceutical and biomedical analysis 47: 59-67.

DeJong JM, Liu Y, Bollon AP, Long RM, Jennewein S, Williams D, Croteau RB 2006. Genetic engineering of taxol biosynthetic genes in Saccharomyces cerevisiae. Biotechnology and bioengineering 93: 212-224.

Delattin N, Cammue BP, Thevissen K 2014. Reactive oxygen species-inducing antifungal agents and their activity against fungal biofilms. Future medicinal chemistry 6: 77-90.

Denning DW 2003. Echinocandin antifungal drugs. The Lancet 362: 1142-1151.

Denning DW, Bromley MJ 2015. How to bolster the antifungal pipeline. Science 347: 1414-1416. 
Devirgiliis C, Murgia C, Danscher G, Perozzi G 2004. Exchangeable zinc ions transiently accumulate in a vesicular compartment in the yeast Saccharomyces cerevisiae. Biochemical and biophysical research communications 323: 58-64.

Dias DA, Urban S, Roessner U 2012. A historical overview of natural products in drug discovery. Metabolites 2: 303-336.

Dinis TC, Madeira VM, Almeida LM 1994. Action of phenolic derivatives (acetaminophen, salicylate, and 5-aminosalicylate) as inhibitors of membrane lipid peroxidation and as peroxyl radical scavengers. Archives of biochemistry and biophysics 315: 161-169.

Dixon SJ, Stockwell BR 2014. The role of iron and reactive oxygen species in cell death. Nat Chem Biol 10: 9-17. doi: 10.1038/nchembio.1416

Dube DH, Bertozzi CR 2005. Glycans in cancer and inflammation-potential for therapeutics and diagnostics. Nature Reviews Drug Discovery 4: 477-488.

Eide DJ 2006. Zinc transporters and the cellular trafficking of zinc. Biochimica et Biophysica Acta (BBA)-Molecular Cell Research 1763: 711-722.

Eliopoulos GM, Perea S, Patterson TF 2002. Antifungal resistance in pathogenic fungi. Clinical Infectious Diseases 35: 1073-1080.

Ellis CD 2004. Characterization and functional significance of Msc2p \& Zrg17p, which form a zinc transport complex in the endoplasmic reticulum of Saccharomyces cerevisiae. [University of Missouri-Columbia.

Elmastaş M, Gedikli F 2010. Total phenolic compounds and antioxidant capacity of leaf, dry fruit and fresh fruit of feijoa (Acca sellowiana, Myrtaceae). J Med Plant Res 4: 1065-1072.

Endo A, Kakiki K, Misato T 1970. Mechanism of action of the antifugal agent polyoxin D. Journal of Bacteriology 104: 189-196.

Farag MA, Porzel A, Wessjohann LA 2012. Comparative metabolite profiling and fingerprinting of medicinal licorice roots using a multiplex approach of GC-MS, LC-MS and 1D NMR techniques. Phytochemistry 76: 60-72.

Ferlay J, Soerjomataram I, Dikshit R, Eser S, Mathers C, Rebelo M, Parkin DM, Forman D, Bray F 2015. Cancer incidence and mortality worldwide: sources, methods and major patterns in GLOBOCAN 2012. International Journal of Cancer 136: E359-E386.

Fernandes KE, Carter DA 2017. The antifungal activity of lactoferrin and its derived peptides: mechanisms of action and synergy with drugs against fungal pathogens. Frontiers in microbiology 8.

Fernandez X, Loiseau A-M, Poulain S, Lizzani-Cuvelier L, Monnier Y 2004. Chemical composition of the essential oil from Feijoa (Feijoa sellowiana Berg.) peel. Journal of Essential Oil Research 16: 274275.

Fernie AR, Trethewey RN, Krotzky AJ, Willmitzer L 2004. Metabolite profiling: from diagnostics to systems biology. Nature reviews molecular cell biology 5: 763-769.

Fleming A 1929. On the Antibacterial Action of Cultures of a Penicillium, with Special Reference to their Use in the Isolation of B. influenzæ. Brit J Exp Pathol 10: 10. 
Folch J, Lees M, Sloane-Stanley G 1957. A simple method for the isolation and purification of total lipids from animal tissues. J biol Chem 226: 497-509.

Foo LY, Watson RR. 2013. Feijoa fruit extract. In: Google Patents.

Fornari T, Vicente G, Vázquez E, García-Risco MR, Reglero G 2012. Isolation of essential oil from different plants and herbs by supercritical fluid extraction. Journal of Chromatography A 1250: 34-48.

Foucquier J, Guedj M 2015. Analysis of drug combinations: current methodological landscape. Pharmacology research \& perspectives 3.

Foury F 1997. Human genetic diseases: a cross-talk between man and yeast. Gene 195: 1-10.

Franklin RB, Feng P, Milon B, Desouki MM, Singh KK, Kajdacsy-Balla A, Bagasra O, Costello LC 2005. hZIP1 zinc uptake transporter down regulation and zinc depletion in prostate cancer. Molecular cancer 4: 32 .

Fresco P, Borges F, Diniz C, Marques M 2006. New insights on the anticancer properties of dietary polyphenols. Medicinal research reviews 26: 747-766.

Fresco P, Borges F, Marques M, Diniz C 2010. The anticancer properties of dietary polyphenols and its relation with apoptosis. Current pharmaceutical design 16: 114-134.

Fröhlich F, Petit C, Kory N, Christiano R, Hannibal-Bach H-K, Graham M, Liu X, Ejsing CS, Farese Jr RV, Walther TC 2015. The GARP complex is required for cellular sphingolipid homeostasis. Elife 4: $\mathrm{e} 08712$.

Fryer LG, Parbu-Patel A, Carling D 2002. The anti-diabetic drugs rosiglitazone and metformin stimulate AMP-activated protein kinase through distinct signaling pathways. Journal of Biological Chemistry 277: 25226-25232.

Gangadevi V, Muthumary J 2008. Taxol, an anticancer drug produced by an endophytic fungus Bartalinia robillardoides Tassi, isolated from a medicinal plant, Aegle marmelos Correa ex Roxb. World Journal of Microbiology and Biotechnology 24: 717.

Gao X, Campian JL, Qian M, Sun X-F, Eaton JW 2009. Mitochondrial DNA damage in iron overload. Journal of Biological Chemistry 284: 4767-4775.

GD RMM, Pippi B, Dalla Lana DF, Amaral AP, Teixeira ML, Souza KC, Fuentefria AM 2016. Reversal of fluconazole resistance induced by a synergistic effect with Acca sellowiana in Candida glabrata strains. Pharm Biol 54: 2410-2419. doi: 10.3109/13880209.2016.1158286

Georgatsou E, Alexandraki D 1994. Two distinctly regulated genes are required for ferric reduction, the first step of iron uptake in Saccharomyces cerevisiae. Molecular and Cellular Biology 14: 3065-3073.

Ghannoum M, Isham N, Verma A, Plaum S, Fleischer A, Hardas B 2013. In vitro antifungal activity of naftifine hydrochloride against dermatophytes. Antimicrobial agents and chemotherapy 57: 4369-4372.

Giaever G, Nislow C 2014. The yeast deletion collection: a decade of functional genomics. Genetics 197: 451-465.

Giaever G, Shoemaker DD, Jones TW, Liang H, Winzeler EA, Astromoff A, Davis RW 1999. Genomic profiling of drug sensitivities via induced haploinsufficiency. Nature genetics 21: 278-283. 
Giedraitiene A, Vitkauskiene A, Naginiene R, Pavilonis A 2011. Antibiotic resistance mechanisms of clinically important bacteria. Medicina (Kaunas) 47: 137-146.

Gietz RD, Schiestl RH 2007. High-efficiency yeast transformation using the LiAc/SS carrier DNA/PEG method. Nature protocols 2: 31-34.

Graack H-R, Wittmann-Liebold B 1998. Mitochondrial ribosomal proteins (MRPs) of yeast. Biochemical Journal 329: 433-448.

Grau S, Pozo J, Romá E, Salavert M, Barrueta J, Peral C, Rodriguez I, Rubio-Rodríguez D, RubioTerrés C 2015. Cost-effectiveness of three echinocandins and fluconazole in the treatment of candidemia and/or invasive candidiasis in nonneutropenic adult patients. ClinicoEconomics and outcomes research: CEOR 7: 527.

Gupta AK, Ryder JE, Cooper EA 2008. Naftifine: a review. Journal of cutaneous medicine and surgery 12: 51-58.

Gupta AK, Versteeg SG 2016. Tavaborole-a treatment for onychomycosis of the toenails. Expert Review of Clinical Pharmacology 9: 1145-1152.

Hamed I, Özogul F, Özogul Y, Regenstein JM 2015. Marine Bioactive compounds and their health benefits: A review. Comprehensive Reviews in Food Science and Food Safety 14: 446-465.

Hap S, Gutierrez NA 2012. Functional properties of some New Zealand fruit extracts towards selected probiotic and pathogenic bacteria. Benef Microbes 3: 309-318. doi: 10.3920/bm2012.0004

Harvey AL, Edrada-Ebel R, Quinn RJ 2015. The re-emergence of natural products for drug discovery in the genomics era. Nature Reviews Drug Discovery 14: 111-129.

Heimark L, Shipkova P, Greene J, Munayyer H, Yarosh-Tomaine T, DiDomenico B, Hare R, Pramanik BN 2002. Mechanism of azole antifungal activity as determined by liquid chromatographic/mass spectrometric monitoring of ergosterol biosynthesis. Journal of mass spectrometry 37: 265-269.

Heitman J 2011. Microbial pathogens in the fungal kingdom. Fungal biology reviews 25: 48-60.

Hider RC, Liu ZD, Khodr HH 2001. Metal chelation of polyphenols. Methods in enzymology 335: 190203.

Hood K, West L, Northcote P, Berridge M, Miller J 2001. Induction of apoptosis by the marine sponge (Mycale) metabolites, mycalamide A and pateamine. Apoptosis 6: 207-219.

Hosiner D, Gerber S, Lichtenberg-Frate H, Glaser W, Schüller C, Klipp E 2014. Impact of acute metal stress in Saccharomyces cerevisiae. PloS one 9: e83330.

Hossain MA, Ghannoum MA 2000. New investigational antifungal agents for treating invasive fungal infections. Expert opinion on investigational drugs 9: 1797-1813.

Huang D-W, Chang W-C, Wu JS-B, Shih R-W, Shen S-C 2016. Vescalagin from pink wax apple [Syzygium samarangense (Blume) Merrill and Perry] alleviates hepatic insulin resistance and ameliorates glycemic metabolism abnormality in rats fed a high-fructose diet. Journal of agricultural and food chemistry 64: 1122-1129.

Hughes AL, Hughes CE, Henderson KA, Yazvenko N, Gottschling DE 2016. Selective sorting and destruction of mitochondrial membrane proteins in aged yeast. Elife 5: e13943. 
Huh W-K, Falvo JV, Gerke LC, Carroll AS, Howson RW, Weissman JS, O'Shea EK 2003. Global analysis of protein localization in budding yeast. Nature 425: 686-691.

Hwang EI, Lee YM, Lee SM, Yeo WH, Moon JS, Kang TH, Park KD, Kim SU 2007. Inhibition of chitin synthase 2 and antifungal activity of lignans from the stem bark of Lindera erythrocarpa. Planta medica 73: 679-682.

Hwang JH, Hwang I-s, Liu Q-H, Woo E-R, Lee DG 2012. (+)-Medioresinol leads to intracellular ROS accumulation and mitochondria-mediated apoptotic cell death in Candida albicans. Biochimie 94: 17841793.

Ibrahim A, Spellberg B, Edwards Jr J 2008. Iron acquisition: A novel prospective on mucormycosis pathogenesis and treatment. Current opinion in infectious diseases 21: 620.

Jayawardena R, Ranasinghe P, Galappatthy P, Malkanthi R, Constantine G, Katulanda P 2012. Effects of zinc supplementation on diabetes mellitus: a systematic review and meta-analysis. Diabetology \& metabolic syndrome 4: 13.

Jia J, Zhu F, Ma X, Cao ZW, Li YX, Chen YZ 2009. Mechanisms of drug combinations: interaction and network perspectives. Nature Reviews Drug Discovery 8: 111-128.

Jonikas MC, Collins SR, Denic V, Oh E, Quan EM, Schmid V, Weibezahn J, Schwappach B, Walter P, Weissman JS 2009. Comprehensive characterization of genes required for protein folding in the endoplasmic reticulum. Science 323: 1693-1697.

Kahn SE, Hull RL, Utzschneider KM 2006. Mechanisms linking obesity to insulin resistance and type 2 diabetes. Nature 444: 840-846.

Kanafani ZA, Perfect JR 2008. Antimicrobial resistance: resistance to antifungal agents: mechanisms and clinical impact. Clin Infect Dis 46: 120-128. doi: 10.1086/524071

Karamać M 2009. Chelation of $\mathrm{Cu}$ (II), Zn (II), and Fe (II) by tannin constituents of selected edible nuts. International journal of molecular sciences 10: 5485-5497.

Karami M, Saeidnia S, Nosrati A 2013. Study of the hepatoprotective activity of methanolic extract of Feijoa sellowiana fruits against MDMA using the isolated rat liver perfusion system. Iran J Pharmaceut Res 12: 85.

Katz L, Baltz RH 2016. Natural product discovery: past, present, and future. Journal of industrial microbiology \& biotechnology 43: 155-176.

Kayikci Ö, Nielsen J 2015. Glucose repression in Saccharomyces cerevisiae. FEMS yeast research 15: fov068.

Khan A, Ahmad A, Akhtar F, Yousuf S, Xess I, Khan LA, Manzoor N 2011. Induction of oxidative stress as a possible mechanism of the antifungal action of three phenylpropanoids. FEMS yeast research 11: $114-122$.

Khan MSA, Ahmad I, Aqil F, Owais M, Shahid M, Musarrat J. 2010. Virulence and pathogenicity of fungal pathogens with special reference to Candida albicans. In. Combating Fungal Infections: Springer. p. 21-45.

Khan ZK, Jain P 2000. Antifungal agents and immunomodulators in systemic mycoses. INDIAN JOURNAL OF CHEST DISEASES AND ALLIED SCIENCES 42: 345-356. 
Khosla C, Keasling JD 2003. Metabolic engineering for drug discovery and development. Nature Reviews Drug Discovery 2: 1019-1025.

Khurana V, Tardiff DF, Chung CY, Lindquist S 2015. Toward stem cell-based phenotypic screens for neurodegenerative diseases. Nature Reviews Neurology 11: 339-350.

Killeen DP, Sansom CE, Lill RE, Eason JR, Gordon KC, Perry NB 2013. Quantitative Raman spectroscopy for the analysis of carrot bioactives. Journal of agricultural and food chemistry 61: 27012708.

Klinger SC, Siupka P, Nielsen MS 2015. Retromer-mediated trafficking of transmembrane receptors and transporters. Membranes 5: 288-306.

Kubinyi H. 2006. Chemogenomics in drug discovery. In. Chemical Genomics: Springer. p. 1-19.

Kurat CF, Natter K, Petschnigg J, Wolinski H, Scheuringer K, Scholz H, Zimmermann R, Leber R, Zechner R, Kohlwein SD 2006. Obese yeast: triglyceride lipolysis is functionally conserved from mammals to yeast. Journal of Biological Chemistry 281: 491-500.

Kwok EY, Severance S, Kosman DJ 2006. Evidence for iron channeling in the Fet3p-Ftr1p high-affinity iron uptake complex in the yeast plasma membrane. Biochemistry 45: 6317-6327.

Lai Y-W, Campbell LT, Wilkins MR, Pang CNI, Chen S, Carter DA 2016. Synergy and antagonism between iron chelators and antifungal drugs in Cryptococcus. International journal of antimicrobial agents 48: 388-394.

Landete J 2011. Ellagitannins, ellagic acid and their derived metabolites: a review about source, metabolism, functions and health. Food Research International 44: 1150-1160.

Laniado-Laborín R, Cabrales-Vargas MN 2009. Amphotericin B: side effects and toxicity. Revista iberoamericana de micología 26: 223-227.

Lapčík O, Klejdus B, Kokoška L, Davidová M, Afandi K, Kubáň V, Hampl R 2005. Identification of isoflavones in Acca sellowiana and two Psidium species (Myrtaceae). Biochem syst Ecol 33: 983-992.

Lee AY, Onge RPS, Proctor MJ, Wallace IM, Nile AH, Spagnuolo PA, Jitkova Y, Gronda M, Wu Y, Kim MK 2014. Mapping the cellular response to small molecules using chemogenomic fitness signatures. Science 344: 208-211.

Lemmon E, McLinden M, Friend D, Linstrom P, Mallard W 2015. NIST chemistry WebBook, NIST standard reference database number 69. National Institute of Standards and Technology, Gaithersburg.

Lesage G, Sdicu A-M, Ménard P, Shapiro J, Hussein S, Bussey H 2004. Analysis of $\beta$-1, 3-glucan assembly in Saccharomyces cerevisiae using a synthetic interaction network and altered sensitivity to caspofungin. Genetics 167: 35-49.

Li L, Kaplan J 2001. The yeast gene MSC2, a member of the cation diffusion facilitator family, affects the cellular distribution of zinc. Journal of Biological Chemistry 276: 5036-5043.

Li XC, Ferreira D, Jacob MR, Zhang Q, Khan SI, ElSohly HN, Nagle DG, Smillie TJ, Khan IA, Walker LA, Clark AM 2004. Antifungal cyclopentenediones from Piper coruscans. J Am Chem Soc 126: 68726873. doi: $10.1021 / \mathrm{ja} 048081 \mathrm{c}$ 
Li Z, Vizeacoumar FJ, Bahr S, Li J, Warringer J, Vizeacoumar FS, Min R, VanderSluis B, Bellay J, DeVit M 2011. Systematic exploration of essential yeast gene function with temperature-sensitive mutants. Nature biotechnology 29: 361-367.

Lightman A, Brandes JM, Binur N, Drugan A, Zinder O 1986. Use of the serum copper/zinc ratio in the differential diagnosis of ovarian malignancy. Clinical chemistry 32: 101-103.

Lill R, Hoffmann B, Molik S, Pierik AJ, Rietzschel N, Stehling O, Uzarska MA, Webert H, Wilbrecht C, Mühlenhoff U 2012. The role of mitochondria in cellular iron-sulfur protein biogenesis and iron metabolism. Biochimica et Biophysica Acta (BBA)-Molecular Cell Research 1823: 1491-1508.

Linton CJ, Borman AM, Cheung G, Holmes AD, Szekely A, Palmer MD, Bridge PD, Campbell CK, Johnson EM 2007. Molecular identification of unusual pathogenic yeast isolates by large ribosomal subunit gene sequencing: 2 years of experience at the United Kingdom mycology reference laboratory. Journal of clinical microbiology 45: 1152-1158.

Lipińska L, Klewicka E, Sójka M 2014. The structure, occurrence and biological activity of ellagitannins: a general review. ACTA Scientiarum Polonorum Technologia Alimentaria 13: 289-299.

Liu Y, Solis NV, Heilmann CJ, Phan QT, Mitchell AP, Klis FM, Filler SG 2014. Role of retrograde trafficking in stress response, host cell interactions, and virulence of Candida albicans. Eukaryotic cell 13: 279-287.

Lopez-Ribot JL, McAtee RK, Lee LN, Kirkpatrick WR, White TC, Sanglard D, Patterson TF 1998. Distinct Patterns of Gene Expression Associated with Development of Fluconazole Resistance in Serial Candida albicansIsolates from Human Immunodeficiency Virus-Infected Patients with Oropharyngeal Candidiasis. Antimicrobial agents and chemotherapy 42: 2932-2937.

Lordan S, Ross RP, Stanton C 2011. Marine bioactives as functional food ingredients: potential to reduce the incidence of chronic diseases. Marine Drugs 9: 1056-1100.

Lui GY, Obeidy P, Ford SJ, Tselepis C, Sharp DM, Jansson PJ, Kalinowski DS, Kovacevic Z, Lovejoy DB, Richardson DR 2013. The iron chelator, deferasirox, as a novel strategy for cancer treatment: oral activity against human lung tumor xenografts and molecular mechanism of action. Molecular pharmacology 83: 179-190.

M Chaudhary P, G Tupe S, V Deshpande M 2013. Chitin synthase inhibitors as antifungal agents. Mini reviews in medicinal chemistry 13: 222-236.

MacDiarmid CW, Gaither LA, Eide D 2000. Zinc transporters that regulate vacuolar zinc storage in Saccharomyces cerevisiae. The EMBO journal 19: 2845-2855.

Macreadie IG, Johnson G, Schlosser T, Macreadie PI 2006. Growth inhibition of Candida species and Aspergillus fumigatus by statins. FEMS microbiology letters 262: 9-13.

Malhotra JD, Kaufman RJ 2011. ER stress and its functional link to mitochondria: role in cell survival and death. Cold Spring Harbor perspectives in biology 3: a004424.

Marobbio C, Agrimi G, Lasorsa F, Palmieri F 2003. Identification and functional reconstitution of yeast mitochondrial carrier for S-adenosylmethionine. The EMBO journal 22: 5975-5982.

Martin H, Burgess EJ, Smith WA, McGhie TK, Cooney JM, Lunken RC, de Guzman E, Trower T, Perry NB 2015. JAK2 and AMP-kinase inhibition in vitro by food extracts, fractions and purified phytochemicals. Food Funct 6: 305-312. doi: 10.1039/c4fo00626g 
Marton MJ, DeRisi JL, Bennett HA, Iyer VR, Meyer MR, Roberts CJ, Stoughton R, Burchard J, Slade D, Dai H 1998. Drug target validation and identification of secondary drug target effects using DNA microarrays. Nature medicine 4: 1293-1301.

Marty A, Finkelstein A 1975. Pores formed in lipid bilayer membranes by nystatin, Differences in its one-sided and two-sided action. The Journal of general physiology 65: 515-526.

McChesney JD, Venkataraman SK, Henri JT 2007. Plant natural products: back to the future or into extinction? Phytochemistry 68: 2015-2022.

Mehta R, Kingsbury W, Valenta J, Actor P 1984. Anti-Candida activity of polyoxin: example of peptide transport in yeasts. Antimicrobial agents and chemotherapy 25: 373-374.

Meletiadis J, Pournaras S, Roilides E, Walsh TJ 2010. Defining fractional inhibitory concentration index cutoffs for additive interactions based on self-drug additive combinations, Monte Carlo simulation analysis, and in vitro-in vivo correlation data for antifungal drug combinations against Aspergillus fumigatus. Antimicrobial agents and chemotherapy 54: 602-609.

Mnaimneh S, Davierwala AP, Haynes J, Moffat J, Peng W-T, Zhang W, Yang X, Pootoolal J, Chua G, Lopez A 2004. Exploration of essential gene functions via titratable promoter alleles. Cell 118: 31-44.

Montpetit B, Thorne K, Barrett I, Andrews K, Jadusingh R, Hieter P, Measday V 2005. Genome-wide synthetic lethal screens identify an interaction between the nuclear envelope protein, Apq12p, and the kinetochore in Saccharomyces cerevisiae. Genetics 171: 489-501.

Moser J. 2011. Gas chromatographic analysis of plant sterols. In.

Nakashima H 2001. Biological activity of Feijoa peel extracts. Occasional Papers of the Kagoshima University Research Center for the Pacific Islands 34: 169-175.

Nasef NA, Mehta S, Powell P, Marlow G, Wileman T, Ferguson LR 2015. Extracts of feijoa inhibit tolllike receptor 2 signaling and activate autophagy implicating a role in dietary control of IBD. PloS one 10: $\mathrm{e} 0130910$.

Newman DJ, Cragg GM 2016. Natural products as sources of new drugs from 1981 to 2014. Journal of natural products 79: 629-661.

Ohno A, Oka K, Sakuma C, Okuda H, Fukuhara K 2011. Characterization of tea cultivated at four different altitudes using $1 \mathrm{H}$ NMR analysis coupled with multivariate statistics. Journal of agricultural and food chemistry 59: 5181-5187.

Okamoto K, Shaw JM 2005. Mitochondrial morphology and dynamics in yeast and multicellular eukaryotes. Annu. Rev. Genet. 39: 503-536.

Oki T, TENMYO O, HIRANO M, TOMATSU K, KAMEI H 1990. Pradimicins A, B and C: new antifungal antibiotics. The Journal of antibiotics 43: 763-770.

Olabinri B, Eniyansoro O, Okoronkwo C, Olabinri P, Olaleye M 2010. Evaluation of chelating ability of aqueous extract of Tetracarpidium conophorum (African walnut) in vitro. International journal of applied research in natural products 3: 13-18.

Omar M, Matsuo Y, Maeda H, Saito Y, Tanaka T 2014. New metabolites of C-glycosidic ellagitannin from Japanese oak sapwood. Organic letters 16: 1378-1381. 
Onofrejová L, Vašičková J, Klejdus B, Stratil P, Mišurcová L, Kráčmar S, Kopecký J, Vacek J 2010. Bioactive phenols in algae: The application of pressurized-liquid and solid-phase extraction techniques. Journal of pharmaceutical and biomedical analysis 51: 464-470.

Ooi SL, Shoemaker DD, Boeke JD 2003. DNA helicase gene interaction network defined using synthetic lethality analyzed by microarray. Nature genetics $35: 277-286$.

Pallares R, Liñares J, Vadillo M, Cabellos C, Manresa F, Viladrich PF, Martin R, Gudiol F 1995. Resistance to penicillin and cephalosporin and mortality from severe pneumococcal pneumonia in Barcelona, Spain. New England Journal of Medicine 333: 474-480.

Paluszynski JP, Klassen R, Meinhardt F 2008. Genetic prerequisites for additive or synergistic actions of 5-fluorocytosine and fluconazole in baker's yeast. Microbiology 154: 3154-3164.

Pandey KB, Rizvi SI 2009. Plant polyphenols as dietary antioxidants in human health and disease. Oxidative medicine and cellular longevity 2: 270-278.

Papouskova B, Bernard M, Ottenschlager J, Karban J, Velisek P, Hrbac J, Sykora J, Storch J, Vacek J 2016. A comprehensive LC/MS analysis of novel cyclopentenedione library. Journal of pharmaceutical and biomedical analysis 128: 342-351.

Pappas PG, Rex JH, Sobel JD, Filler SG, Dismukes WE, Walsh TJ, Edwards JE 2004. Guidelines for treatment of candidiasis. Clinical Infectious Diseases 38: 161-189.

Parks LW, Casey WM 1995. Physiological implications of sterol biosynthesis in yeast. Annual Reviews in Microbiology 49: 95-116.

Parsons AB, Brost RL, Ding H, Li Z, Zhang C, Sheikh B, Brown GW, Kane PM, Hughes TR, Boone C 2004. Integration of chemical-genetic and genetic interaction data links bioactive compounds to cellular target pathways. Nature biotechnology 22: 62-69.

Parsons AB, Lopez A, Givoni IE, Williams DE, Gray CA, Porter J, Chua G, Sopko R, Brost RL, Ho CH 2006. Exploring the mode-of-action of bioactive compounds by chemical-genetic profiling in yeast. Cell 126: 611-625.

Perfect JR 2017. The antifungal pipeline: a reality check. Nature Reviews Drug Discovery.

Perrone GG, Tan S-X, Dawes IW 2008. Reactive oxygen species and yeast apoptosis. Biochimica et Biophysica Acta (BBA)-Molecular Cell Research 1783: 1354-1368.

Pfaller MA 2012. Antifungal drug resistance: mechanisms, epidemiology, and consequences for treatment. The American journal of medicine 125: S3-S13.

Pfaller MA, Messer SA, Rhomberg PR, Jones RN, Castanheira M 2016. Activity of a long-acting echinocandin, CD101, determined using CLSI and EUCAST reference methods, against Candida and Aspergillus spp., including echinocandin-and azole-resistant isolates. Journal of Antimicrobial Chemotherapy 71: 2868-2873.

Philpott CC, Protchenko O 2008. Response to iron deprivation in Saccharomyces cerevisiae. Eukaryotic cell 7: 20-27.

Piao M, Liu Y, Yu T, Lu Y 2016. Zinc supplementation ameliorates ER stress and autophagy in liver in a rat model of type 2 diabetes mellitus. Biomedical Research 27. 
Pintard L, Bujnicki JM, Lapeyre B, Bonnerot C 2002. MRM2 encodes a novel yeast mitochondrial 21S rRNA methyltransferase. The EMBO journal 21: 1139-1147.

Plaza M, Santoyo S, Jaime L, Reina GG-B, Herrero M, Señoráns FJ, Ibáñez E 2010. Screening for bioactive compounds from algae. Journal of pharmaceutical and biomedical analysis 51: 450-455.

Prabuseenivasan S, Jayakumar M, Ignacimuthu S 2006. In vitro antibacterial activity of some plant essential oils. BMC complementary and alternative medicine 6: 1 .

Prakash O, Kumar A, Kumar P 2013. Anticancer potential of plants and natural products: a review. American Journal of Pharmacological Sciences 1: 104-115.

Pringle JR 1991. [52] Staining of bud scars and other cell wall chitin with Calcofluor. Methods in enzymology 194: 732-735.

Prosser G, Copp J, Syddall S, Williams E, Smaill J, Wilson W, Patterson A, Ackerley D 2010. Discovery and evaluation of Escherichia coli nitroreductases that activate the anti-cancer prodrug CB1954. Biochemical Pharmacology 79: 678-687.

Puig S, Askeland E, Thiele DJ 2005. Coordinated remodeling of cellular metabolism during iron deficiency through targeted mRNA degradation. Cell 120: 99-110.

Ray J. 1724. Synopsis methodica stirpium Britannicarum: Innys.

Razzaghi-Abyaneh M, Rai M. 2013. Antifungal metabolites from plants: Springer.

Rees EM, Lee J, Thiele DJ 2004. Mobilization of intracellular copper stores by the ctr2 vacuolar copper transporter. Journal of Biological Chemistry 279: 54221-54229.

Rice-evans CA, Miller NJ, Bolwell PG, Bramley PM, Pridham JB 1995. The relative antioxidant activities of plant-derived polyphenolic flavonoids. Free radical research 22: 375-383.

Ripsin CM, Kang H, Urban RJ 2009. Management of blood glucose in type 2 diabetes mellitus. Am Fam Physician 79: 29-36.

Robinson DG, Chen W, Storey JD, Gresham D 2014. Design and analysis of Bar-seq experiments. G3: Genes| Genomes| Genetics 4: 11-18.

Robu S, Aprotosoaie A, Miron A, CIOANCĂ O, STĂNESCU U, HĂNCIANU M 2012. In vitro antioxidant activity of ethanolic extracts from some Lavandula species cultivated in Romania. Cell 60: 3.

Rossi A, Rigano D, Pergola C, Formisano C, Basile A, Bramanti P, Senatore F, Sautebin L 2007. Inhibition of inducible nitric oxide synthase expression by an acetonic extract from Feijoa sellowiana Berg. fruits. Journal of agricultural and food chemistry 55: 5053-5061.

Rouault TA 2012. Biogenesis of iron-sulfur clusters in mammalian cells: new insights and relevance to human disease. Disease models \& mechanisms 5: 155-164.

Ruggles KV, Garbarino J, Liu Y, Moon J, Schneider K, Henneberry A, Billheimer J, Millar JS, Marchadier D, Valasek MA 2014. A functional, genome-wide evaluation of liposensitive yeast identifies the "ARE2 required for viability"(ARV1) gene product as a major component of eukaryotic fatty acid resistance. Journal of Biological Chemistry 289: 4417-4431. 
Ruiz-Herrera J, San-Blas G 2003. Chitin synthesis as a target for antifungal drugs. Current Drug TargetsInfectious Disorders 3: 77-91.

Rustici G, van Bakel H, Lackner DH, Holstege FC, Wijmenga C, Bähler J, Brazma A 2007. Global transcriptional responses of fission and budding yeast to changes in copper and iron levels: a comparative study. Genome biology 8: R73.

Rutherford JC, Bird AJ 2004. Metal-responsive transcription factors that regulate iron, zinc, and copper homeostasis in eukaryotic cells. Eukaryotic cell 3: 1-13.

Saj O, Roy R, Savitha S 2008. Chemical composition and antimicrobial properties of essential oil of Feijoa sellowiana O. Berg.(pineapple guava). J pure appl microbiol 2: 227-230.

Sambrook J, Russell DW 2006. Preparation of plasmid DNA by alkaline lysis with SDS: minipreparation. Cold Spring Harbor Protocols 2006: pdb. prot4084.

San-Blas G, Calderone RA. 2008. Pathogenic fungi: insights in molecular biology: Horizon Scientific Press.

Santos R, Dancis A, David E, Camadro J-M, Lesuisse E 2003. Zinc suppresses the iron-accumulation phenotype of Saccharomyces cerevisiae lacking the yeast frataxin homologue (Yfh1). Biochemical Journal 375: 247-254.

Sassi HE, Bastajian N, Kainth P, Andrews BJ 2009. Reporter-based synthetic genetic array analysis: a functional genomics approach for investigating the cell cycle in Saccharomyces cerevisiae. Yeast Functional Genomics and Proteomics: Methods and Protocols: 55-73.

Satish S, Mohana D, Ranhavendra M, Raveesha K 2007. Antifungal activity of some plant extracts against important seed borne pathogens of Aspergillus sp. An International Journal of Agricultural Technology 3: 109-119.

Saunders C, Parsonson-Ensor C, Greer G 2011. Viability of a commercially sustainable West Coast horticulture industry.

Sburlati A, Cabib E 1986. Chitin synthetase 2, a presumptive participant in septum formation in Saccharomyces cerevisiae. Journal of Biological Chemistry 261: 15147-15152.

Scalbert A, Johnson IT, Saltmarsh M 2005. Polyphenols: antioxidants and beyond. The American journal of clinical nutrition 81 : 215S-217S.

Schwartz RE, Sesin DF, Joshua H, Wilson KE, Kempf AJ, Goklen KA, Kuehner D, Gailliot P, Gleason C, WHITE R 1992. Pneumocandins from Zalerion arboricola. The Journal of antibiotics 45: 1853-1866.

Seaman MN, Marcusson EG, Cereghino JL, Emr SD 1997. Endosome to Golgi retrieval of the vacuolar protein sorting receptor, Vps10p, requires the function of the VPS29, VPS30, and VPS35 gene products. The Journal of cell biology 137: 79-92.

Seto B 2012. Rapamycin and mTOR: a serendipitous discovery and implications for breast cancer. Clinical and translational medicine 1: 1 .

Sevcikova Z, Pour M, Novak D, Ulrichova J, Vacek J 2014. Chemical properties and biological activities of cyclopentenediones: A Review. Mini reviews in medicinal chemistry 14: 322-331.

Shan Y. 2016. Comprehensive Utilization of Citrus By-products: Academic Press. 
Shander A, Cappellini M, Goodnough L 2009. Iron overload and toxicity: the hidden risk of multiple blood transfusions. Vox sanguinis 97: 185-197.

Shaw GJ, Allen JM, Yates MK 1989. Volatile flavour constituents in the skin oil from Feijoa sellowiana. Phytochemistry 28: 1529-1530.

Shaw GJ, Allen JM, Yates MK, Franich RA 1990. Volatile flavour constituents of feijoa (Feijoa sellowiana) - analysis of fruit flesh. Journal of the Science of Food and Agriculture 50: 357-361.

Sheih I-C, Wu T-K, Fang TJ 2009. Antioxidant properties of a new antioxidative peptide from algae protein waste hydrolysate in different oxidation systems. Bioresource Technology 100: 3419-3425.

Shibata T, Takahashi T, Yamada E, Kimura A, Nishikawa H, Hayakawa H, Nomura N, Mitsuyama J 2012. T-2307 causes collapse of mitochondrial membrane potential in yeast. Antimicrobial agents and chemotherapy 56: 5892-5897.

Shin S 2004. Essential oil compounds from Agastache rugosa as antifungal agents against Trichophyton species. Archives of pharmacal research 27: 295-299.

Shin S, Kang CA 2003. Antifungal activity of the essential oil of Agastache rugosa Kuntze and its synergism with ketoconazole. Letters in applied microbiology 36: 111-115.

Shin S, Pyun MS 2004. Anti-Candida effects of estragole in combination with ketoconazole or amphotericin B. Phytotherapy Research 18: 827-830.

Sigle HC, Thewes S, Niewerth M, Korting HC, Schafer-Korting M, Hube B 2005. Oxygen accessibility and iron levels are critical factors for the antifungal action of ciclopirox against Candida albicans. $\mathrm{J}$ Antimicrob Chemother 55: 663-673. doi: 10.1093/jac/dki089

Simm C, Lahner B, Salt D, LeFurgey A, Ingram P, Yandell B, Eide DJ 2007. Saccharomyces cerevisiae vacuole in zinc storage and intracellular zinc distribution. Eukaryotic cell 6: 1166-1177.

Simon JA, Bedalov A 2004. Yeast as a model system for anticancer drug discovery. Nature Reviews Cancer 4: 481-487.

Singh A, Kaur N, Kosman DJ 2007. The metalloreductase Fre6p in Fe-efflux from the yeast vacuole. Journal of Biological Chemistry 282: 28619-28626.

Singh G, Marimuthu P, de Heluani CS, Catalan C 2005. Chemical constituents and antimicrobial and antioxidant potentials of essential oil and acetone extract of Nigella sativa seeds. Journal of the Science of Food and Agriculture 85: 2297-2306.

Sitepu I, Ignatia L, Franz A, Wong D, Faulina S, Tsui M, Kanti A, Boundy-Mills K 2012. An improved high-throughput Nile red fluorescence assay for estimating intracellular lipids in a variety of yeast species. Journal of microbiological methods 91: 321-328.

Slinker BK 1998. The statistics of synergism. Journal of molecular and cellular cardiology 30: 723-731.

Small SA, Petsko GA 2015. Retromer in Alzheimer disease, Parkinson disease and other neurological disorders. Nature Reviews Neuroscience 16: 126-132.

Smith AM, Heisler LE, Onge RPS, Farias-Hesson E, Wallace IM, Bodeau J, Harris AN, Perry KM, Giaever G, Pourmand N 2010. Highly-multiplexed barcode sequencing: an efficient method for parallel analysis of pooled samples. Nucleic acids research: gkq368. 
Soares EV, Hebbelinck K, Soares HM 2003. Toxic effects caused by heavy metals in the yeast Saccharomyces cerevisiae: a comparative study. Canadian journal of microbiology 49: 336-343.

Song S, Tang Q, Hayat K, Karangwa E, Zhang X, Xiao Z 2014. Effect of enzymatic hydrolysis with subsequent mild thermal oxidation of tallow on precursor formation and sensory profiles of beef flavours assessed by partial least squares regression. Meat science 96: 1191-1200.

Sopko R, Huang D, Preston N, Chua G, Papp B, Kafadar K, Snyder M, Oliver SG, Cyert M, Hughes TR 2006. Mapping pathways and phenotypes by systematic gene overexpression. Molecular cell 21: 319-330.

Strobel GA, Spang S, Kluck K, Hess W, Sears J, Livinghouse T 2008. Synergism among volatile organic compounds resulting in increased antibiosis in Oidium sp. The FEMS microbiology letters 283: 140145 .

Sumner LW, Amberg A, Barrett D, Beale MH, Beger R, Daykin CA, Fan TW-M, Fiehn O, Goodacre R, Griffin JL 2007. Proposed minimum reporting standards for chemical analysis. Metabolomics 3: 211221.

Sunderman F 1975. Electrothermal atomic absorption spectrometry of trace metals in biological fluids. Annals of Clinical \& Laboratory Science 5: 421-434.

Tapiero H, Tew KD 2003. Trace elements in human physiology and pathology: zinc and metallothioneins. Biomedicine \& Pharmacotherapy 57: 399-411.

Thiel R 2010. Systemic mycoses: An overview for modern natural health professionals. The Original Internist 17: 119-128.

Thompson GR, Cadena J, Patterson TF 2009. Overview of antifungal agents. Clinics in chest medicine 30: 203-215.

Tkach JM, Yimit A, Lee AY, Riffle M, Costanzo M, Jaschob D, Hendry JA, Ou J, Moffat J, Boone C 2012. Dissecting DNA damage response pathways by analysing protein localization and abundance changes during DNA replication stress. Nature cell biology 14: 966-976.

Tong AHY, Evangelista M, Parsons AB, Xu H, Bader GD, Page N, Robinson M, Raghibizadeh S, Hogue $\mathrm{CW}$, Bussey H 2001. Systematic genetic analysis with ordered arrays of yeast deletion mutants. Science 294: 2364-2368.

Tong AHY, Lesage G, Bader GD, Ding H, Xu H, Xin X, Young J, Berriz GF, Brost RL, Chang M 2004. Global mapping of the yeast genetic interaction network. Science 303: 808-813.

Toume M, Tani M 2014. Change in activity of serine palmitoyltransferase affects sensitivity to syringomycin E in yeast Saccharomyces cerevisiae. FEMS microbiology letters 358: 64-71.

TUNCEL NB, YILMAZ N, BOZBIYIK NK editors. WORKSHOP ON ANTIOXIDANT MEASUREMENT ASSAY METHODS.

Tuomilehto J, Lindström J, Eriksson JG, Valle TT, Hämäläinen H, Ilanne-Parikka P, KeinänenKiukaanniemi S, Laakso M, Louheranta A, Rastas M 2001. Prevention of type 2 diabetes mellitus by changes in lifestyle among subjects with impaired glucose tolerance. New England Journal of Medicine 344: 1343-1350. 
Turco F, Palumbo I, Andreozzi P, Sarnelli G, De Ruberto F, Esposito G, Basile A, Cuomo R 2016. Acetonic Extract from the Feijoa sellowiana Berg. Fruit Exerts Antioxidant Properties and Modulates Disaccharidases Activities in Human Intestinal Epithelial Cells. Phytother Res 30: 1308-1315. doi: 10.1002/ptr.5629

Van Leeuwen M, Smant W, De Boer W, Dijksterhuis J 2008. Filipin is a reliable in situ marker of ergosterol in the plasma membrane of germinating conidia (spores) of Penicillium discolor and stains intensively at the site of germ tube formation. Journal of microbiological methods 74: 64-73.

Vanden BH, Dromer F, Improvisi I, Lozano-Chiu M, Rex J, Sanglard D 1997. Antifungal drug resistance in pathogenic fungi. Medical mycology 36: 119-128.

Varga A, Molnar J 2000. Biological activity of feijoa peel extracts. Anticancer Res 20: 4323-4330.

Verduyn Lunel FM, Mennink-Kersten MASH, Ruegebrink D, van der Lee HAL, Donnelly JP, Blijlevens NMA, Verweij PE 2009. Value of Candida serum markers in patients with invasive candidiasis after myeloablative chemotherapy. Diagnostic Microbiology and Infectious Disease 64: 408415. doi: https://doi.org/10.1016/j.diagmicrobio.2009.04.012

Vermes A, Guchelaar H-J, Dankert J 2000. Flucytosine: a review of its pharmacology, clinical indications, pharmacokinetics, toxicity and drug interactions. Journal of Antimicrobial Chemotherapy 46: $171-179$.

Vicente M, Basilio A, Cabello A, Peláez F 2003. Microbial natural products as a source of antifungals. Clinical Microbiology and Infection 9: 15-32.

Vivas N, Laguerre M, Pianet de Boissel I, Vivas de Gaulejac N, Nonier M-F 2004. Conformational interpretation of vescalagin and castalagin physicochemical properties. Journal of agricultural and food chemistry 52: 2073-2078.

Vuotto ML, Basile A, Moscatiello V, De Sole P, Castaldo-Cobianchi R, Laghi E, Ielpo MTL 2000. Antimicrobial and antioxidant activities of Feijoa sellowiana fruit. International journal of antimicrobial agents 13: 197-201.

Waghorne CL, Corkran HM, Hunt-Painter AA, Niktab E, Baty JW, Berridge MV, Munkacsi AB, McConnell MJ, Timmer MS, Stocker BL 2016. N, N-Bis (glycityl) amines as anti-cancer drugs. Bioorganic \& medicinal chemistry 24: 3932-3939.

Waller SB, Cleff MB, Serra EF, Silva AL, dos Reis Gomes A, de Mello JRB, de Faria RO, Meireles MCA 2017. Plants from Lamiaceae family as source of antifungal molecules in humane and veterinary medicine. Microbial Pathogenesis.

Warde-Farley D, Donaldson SL, Comes O, Zuberi K, Badrawi R, Chao P, Franz M, Grouios C, Kazi F, Lopes CT 2010. The GeneMANIA prediction server: biological network integration for gene prioritization and predicting gene function. Nucleic acids research 38: W214-W220.

Wardle HM, Law D, Moore CB, Mason C, Denning DW 1995. In vitro activity of D0870 compared with those of other azoles against fluconazole-resistant Candida spp. Antimicrobial agents and chemotherapy 39: 868-871.

Waters BM, Eide DJ 2002. Combinatorial control of yeast FET4 gene expression by iron, zinc, and oxygen. Journal of Biological Chemistry 277: 33749-33757. 
Watson RR, Preedy VR. 2010. Bioactive foods and extracts: Cancer treatment and prevention: CRC Press.

Wattenberg LW, Patterson S, Antonides JD 2010. Chitin or chitin-like glycans as targets for late-term cancer chemoprevention. Cancer Prevention Research 3: 1519-1522.

Weitz D, Weintraub H, Fisher E, Schwartzbard AZ 2010. Fish oil for the treatment of cardiovascular disease. Cardiology in review 18: 258.

Weston RJ 2010. Bioactive products from fruit of the feijoa (Feijoa sellowiana, Myrtaceae): A review. Food Chem 121: 923-926.

Whelan WL 1987. The genetic basis of resistance to 5-fluorocytosine in Candida species and Cryptococcus neoformans. CRC Critical Reviews in Microbiology 15: 45-56.

White TC, Marr KA, Bowden RA 1998. Clinical, cellular, and molecular factors that contribute to antifungal drug resistance. Clinical microbiology reviews 11: 382-402.

Wilson LS, Reyes CM, Stolpman M, Speckman J, Allen K, Beney J 2002. The direct cost and incidence of systemic fungal infections. Value in Health 5: 26-34.

Winzeler E, Lee B, McCusker J, Davis R 1999. Whole genome genetic-typing in yeast using highdensity oligonucleotide arrays. Parasitology 118: 73-80.

Wiśnicka R, Krzepiłko A, Wawryn J, Biliński T 1996. Iron toxicity in yeast. Acta Microbiologica Polonica 46: 339-347.

Wold S, Sjöström M, Eriksson L 2001. PLS-regression: a basic tool of chemometrics. Chemometrics and intelligent laboratory systems 58: 109-130.

Worley B, Powers R 2013. Multivariate analysis in metabolomics. Current Metabolomics 1: 92-107.

Wrede C, Buettner R, Bollheimer L, Schölmerich J, Palitzsch K, Hellerbrand C 2006. Association between serum ferritin and the insulin resistance syndrome in a representative population. European Journal of Endocrinology 154: 333-340.

Wyche TP, Piotrowski JS, Hou Y, Braun D, Deshpande R, Mcllwain S, Ong IM, Myers CL, Guzei IA, Westler WM 2014. Forazoline A: Marine-Derived Polyketide with Antifungal In Vivo Efficacy. Angewandte Chemie 126: 11767-11770.

Xu J, Zhou Q, Xu W, Cai L 2011a. Endoplasmic reticulum stress and diabetic cardiomyopathy. Experimental diabetes research 2012.

Xu L, Meng W, Cao C, Wang J, Shan W, Wang Q 2015. Antibacterial and antifungal compounds from marine fungi. Marine Drugs 13: 3479-3513.

Xu Z, Zhang Y, Fu H, Zhong H, Hong K, Zhu W 2011b. Antifungal quinazolinones from marine-derived Bacillus cereus and their preparation. Bioorganic \& medicinal chemistry letters 21: 4005-4007.

Yamaguchi MU, Garcia FP, Cortez DAG, Ueda-Nakamura T, Dias Filho BP, Nakamura CV 2011. Antifungal effects of Ellagitannin isolated from leaves of Ocotea odorifera (Lauraceae). Antonie van Leeuwenhoek 99: 507-514.

Yan Tong AH, Boone C 2006. Synthetic genetic array analysis in Saccharomyces cerevisiae. Yeast Protocol: 171-191. 
Yan Z, Costanzo M, Heisler LE, Paw J, Kaper F, Andrews BJ, Boone C, Giaever G, Nislow C 2008. Yeast Barcoders: a chemogenomic application of a universal donor-strain collection carrying bar-code identifiers. Nature methods 5: 719-725.

Yao W, Lei G, Yong Y, Xu C, Zhien D 2014. Analysis of Aroma components in Torreya Grandis by Static Headspace-GC/MS. Journal of the Chinese Cereals and Oils Association 7: 025.

Yoon J-H, Baek SJ 2005. Molecular targets of dietary polyphenols with anti-inflammatory properties. Yonsei medical journal 46: 585-596.

Yoon YS, Cho H, LEE JH, Yoon G 2004. Mitochondrial Dysfunction via Disruption of Complex II Activity during Iron Chelation-Induced Senescence-like Growth Arrest of Chang Cells. Annals of the New York Academy of Sciences 1011: 123-132.

Yuan DS 2011. Dithizone staining of intracellular zinc: an unexpected and versatile counterscreen for auxotrophic marker genes in Saccharomyces cerevisiae. PloS one 6: e25830.

Yun B-W, Yan Z, Amir R, Hong S, Jin Y-W, Lee E-K, Loake GJ 2012. Plant natural products: history, limitations and the potential of cambial meristematic cells. Biotechnology and Genetic Engineering Reviews 28: 47-60.

Zarember KA, Cruz AR, Huang C-Y, Gallin JI 2009. Antifungal activities of natural and synthetic iron chelators alone and in combination with azole and polyene antibiotics against Aspergillus fumigatus. Antimicrobial agents and chemotherapy 53: 2654-2656.

Zeng Q, Qiu F, Yuan L 2008. Production of artemisinin by genetically-modified microbes. Biotechnology letters 30: 581-592.

Zhao H, Butler E, Rodgers J, Spizzo T, Duesterhoeft S, Eide D 1998. Regulation of zinc homeostasis in yeast by binding of the ZAP1 transcriptional activator to zinc-responsive promoter elements. Journal of Biological Chemistry 273: 28713-28720.

Zhao H, Eide DJ 1997. Zap1p, a metalloregulatory protein involved in zinc-responsive transcriptional regulation in Saccharomyces cerevisiae. Molecular and Cellular Biology 17: 5044-5052.

Zheng W, Zheng H, Zhao X, Zhang Y, Xie Q, Lin X, Chen A, Yu W, Lu G, Shim WB 2016. Retrograde trafficking from the endosome to the trans-Golgi network mediated by the retromer is required for fungal development and pathogenicity in Fusarium graminearum. New Phytologist 210: 1327-1343.

Zhu H, Bilgin M, Bangham R, Hall D, Casamayor A, Bertone P, Lan N, Jansen R, Bidlingmaier S, Houfek T 2001. Global analysis of protein activities using proteome chips. Science 293: 2101-2105.

Zotchev SB 2003. Polyene macrolide antibiotics and their applications in human therapy. Current medicinal chemistry 10: 211-223.

Zuin A, Gabrielli N, Calvo IA, García-Santamarina S, Hoe K-L, Kim DU, Park H-O, Hayles J, Ayté J, Hidalgo E 2008. Mitochondrial dysfunction increases oxidative stress and decreases chronological life span in fission yeast. PloS one 3: e2842. 


\section{Appendix A Media}

$\underline{\text { SC+HEPES (shown as SC in full thesis) }}$

Yeast nitrogen base (without amino acids)

Monosodium sulfate

Amino acid mixture

Glucose $(40 \%)$

HEPES (1M)

Agar (for SC-agar media only)

$\underline{\text { YPD media }}$

Yeast extract

Peptone

Adenine

Glucose (40\%)

Agar $\underline{1 \text { Liter final volume }}$

$1.36 \mathrm{~g}$

$0.8 \mathrm{~g}$

$2 \mathrm{~g}$

$50 \mathrm{ml}$

$25 \mathrm{ml}$

$16 \mathrm{~g}$

1 Liter final volume

$10 \mathrm{~g}$

$20 \mathrm{~g}$

$0.12 \mathrm{~g}$

$50 \mathrm{ml}$

$20 \mathrm{~g}$
Enriched sporulation media for SGA

Potasium acetate

Glucose (40\%)

Agar

Yeast extract

Amino acid (His/Leu/Lys/Ura)
1 Liter final volume

$10 \mathrm{~g}$

$50 \mathrm{ml}$

$20 \mathrm{~g}$

$1 \mathrm{~g}$

$0.1 \mathrm{~g}$ 


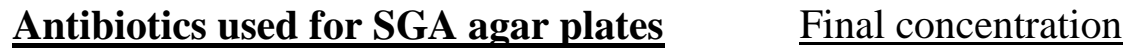

ClonNat

G418

Thialysine

Canavanine
$100 \mu \mathrm{g} / \mathrm{ml}$

$200 \mu \mathrm{g} / \mathrm{ml}$

$50 \mu \mathrm{g} / \mathrm{ml}$

$50 \mu \mathrm{g} / \mathrm{ml}$

\section{Amino acid mixture:}

$3 \mathrm{~g}$ adenine, $2 \mathrm{~g}$ uracil, $2 \mathrm{~g}$ inositol, $0.2 \mathrm{~g}$ para-aminobenzoic acid, $2 \mathrm{~g}$ alanine, $2 \mathrm{~g}$ arginine, 2 g asparagines, $2 \mathrm{~g}$ aspartic acid, $2 \mathrm{~g}$ cysteine, $2 \mathrm{~g}$ glutamic acid, $2 \mathrm{~g}$ glutamine, $2 \mathrm{~g}$ glycine, $2 \mathrm{~g}$ histidine, $2 \mathrm{~g}$ isoleucine, $10 \mathrm{~g}$ leucine, $2 \mathrm{~g}$ lysine, $2 \mathrm{~g}$ methionine, $2 \mathrm{~g}$ phenylalanine, $2 \mathrm{~g}$ proline, $2 \mathrm{~g}$ serine, $2 \mathrm{~g}$ threonine, $2 \mathrm{~g}$ tyrosine, $2 \mathrm{~g}$ tryptophan, $2 \mathrm{~g}$ valine 